\title{
Iterating Meaning - An Analysis of the Digital Conversation Surrounding the Journey of Nishiyuu
}

by

Kathleen Dowdall

A thesis submitted to the Faculty of Graduate and Postdoctoral Affairs in partial fulfillment of the requirements for the degree of

\author{
Master of Arts \\ in \\ Human-Computer Interaction \\ Carleton University \\ Ottawa, Ontario \\ (C) 2014, Kathleen Dowdall
}




\begin{abstract}
This project examines the mechanisms of digital communications media by engaging with theoretical understandings of the nature of digital utterances as applied to the conversation surrounding the 'Journey of Nishiyuu' walk from early 2013. By analyzing and comparing the development of the conversation across platforms, the analysis provides a perspective as to how diverse digital locations are used in unique ways at different times and with fluctuating volumes at various stages in the conversation. This provides insight into the effect of user adaptation on digital communication. The project will also show how the configuration of the conversation can be transformed by the media through which the user accesses information and the platform on which the conversation unfolds. By examining the structure of the conversation, it illustrates how digital communication is inherently iterative; requiring pragmatic, and emphatic iteration and the strategy of segmentation.
\end{abstract}




\section{Acknowledgements}

This work would not have been possible without the support of many people. Thank you to my supervisor, Professor Fai for guiding me through the process, allowing me to pursue my ideas, and reigning me in so that my task was achievable. Professor Biddle provided instrumental assistance retrieving website data. My fellow students in the HCI office provided sounding boards and saved hours of time by sharing their knowledge.

On a personal note, my husband and parents provided the support I needed to enable me to keep up with my studies. I most certainly would not have written this without their help. 


\section{Table of Contents}

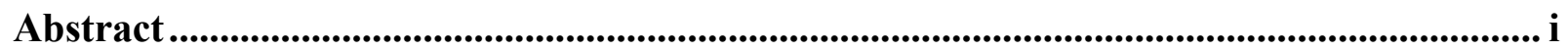

Acknowledgements ........................................................................................................................... ii

Table of Contents ............................................................................................................ ii

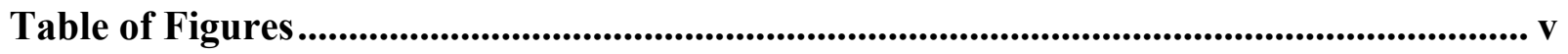

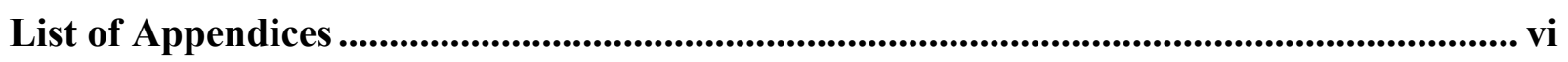

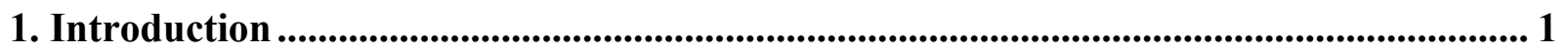

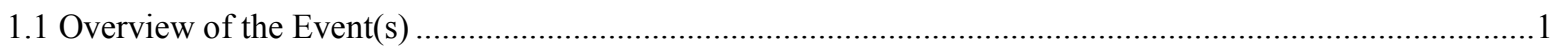

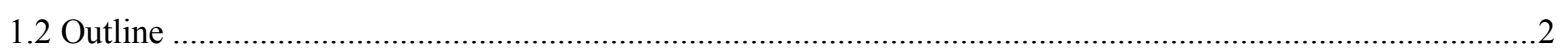

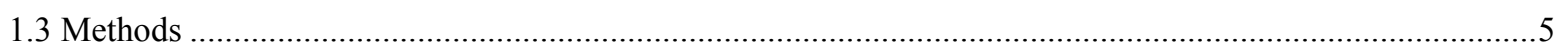

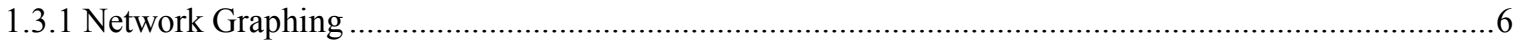

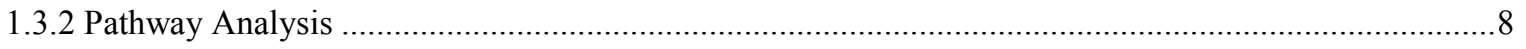

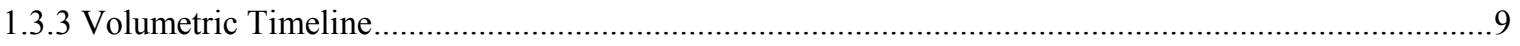

1.3.4 Characteristics of Affordances/Location Observations .........................................................................10

2. Literature Review.................................................................................................................... 12

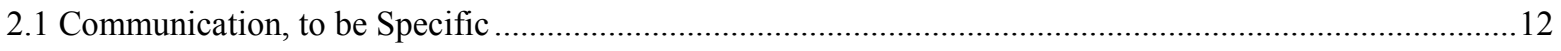

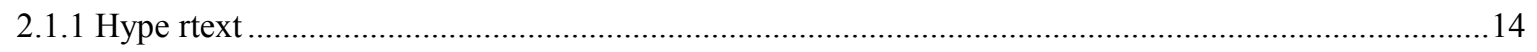

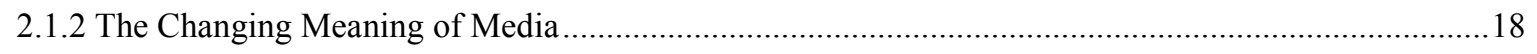

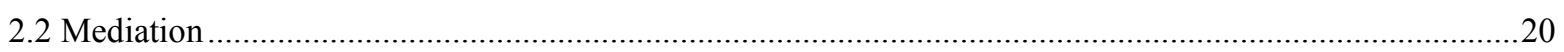

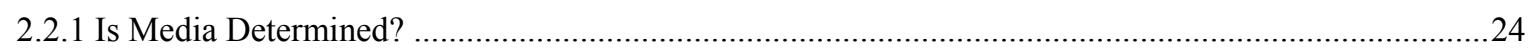

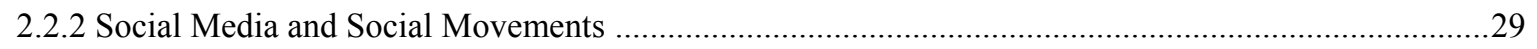

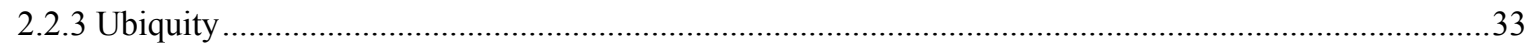

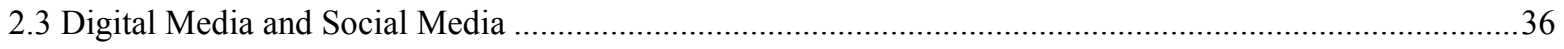

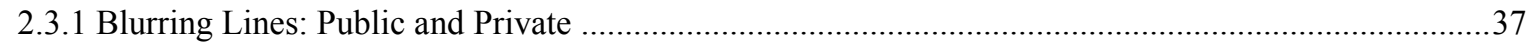

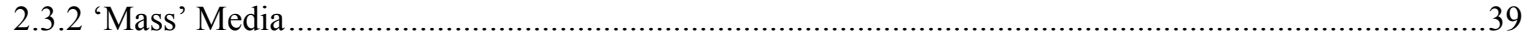

2.3.3 More Blurry Lines: Producer and Consumer...................................................................................44

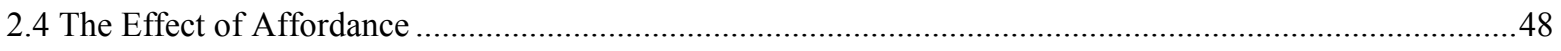

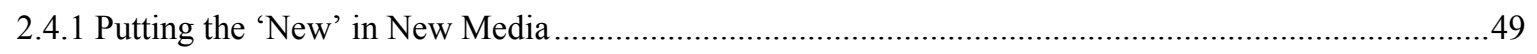

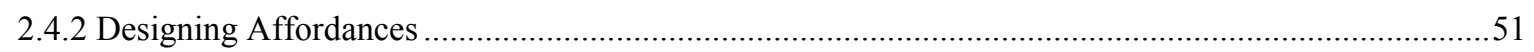

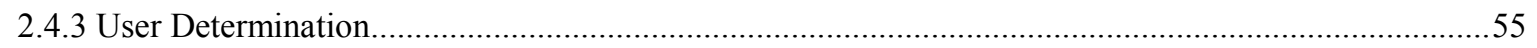

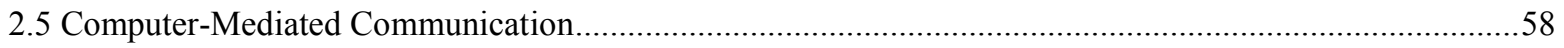

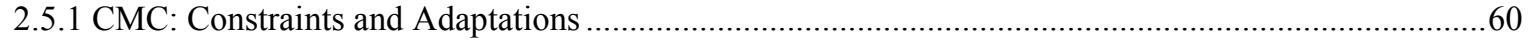

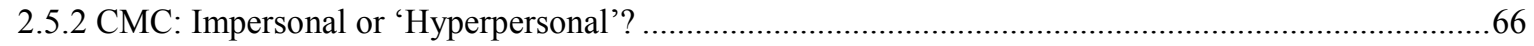

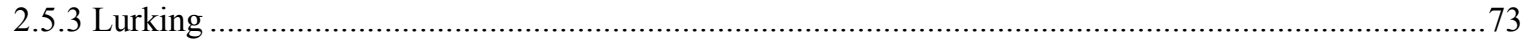

2.5.4 Relating and Creating Our 'Self' Online …...................................................................................... 74 


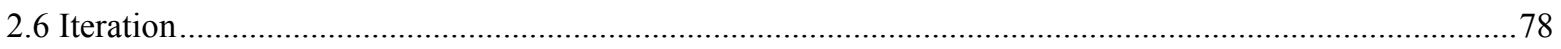

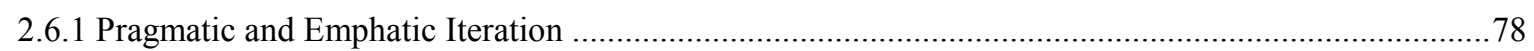

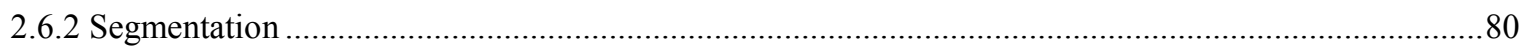

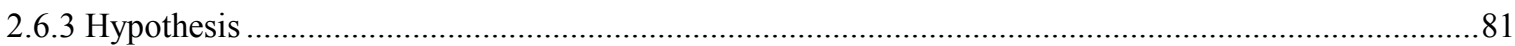

3. Analysis of the Digital Conversation .................................................................................. 82

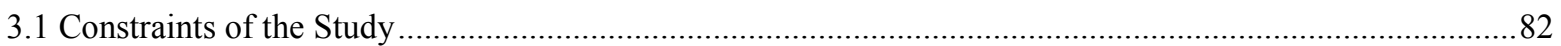

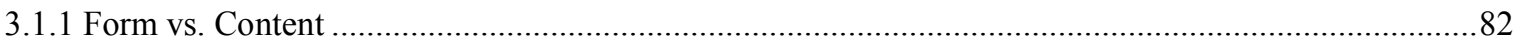

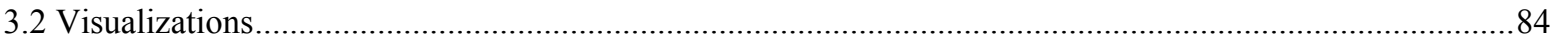

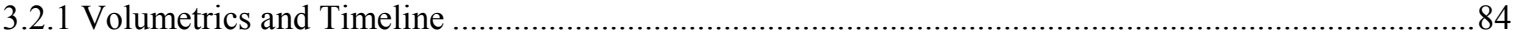

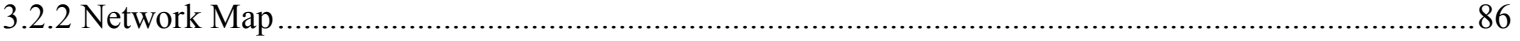

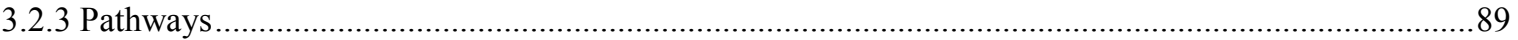

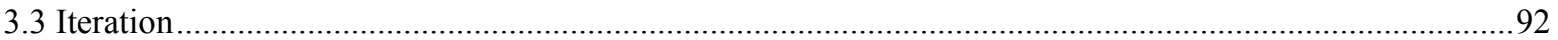

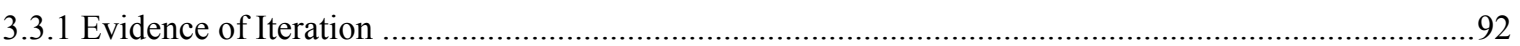

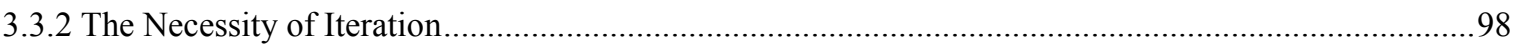

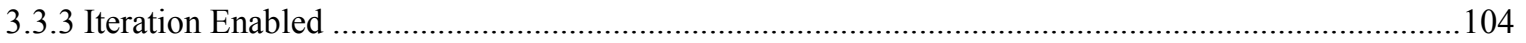

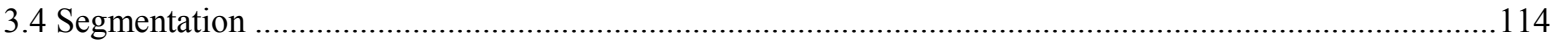

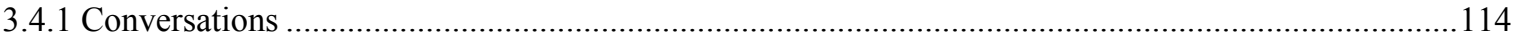

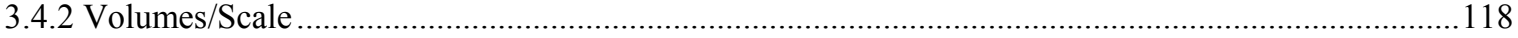

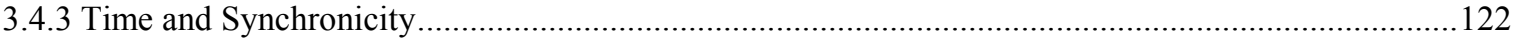

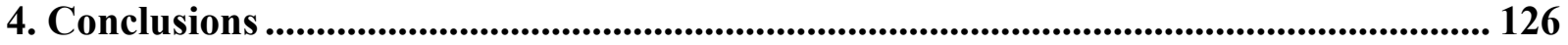

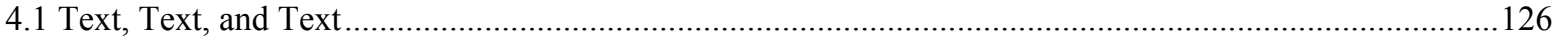

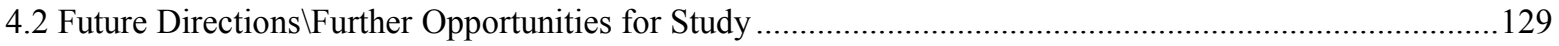

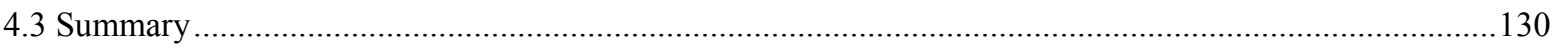

Appendix “A” Network Map ........................................................................................................ 134

Appendix "B" Pathway Charts..................................................................................................... 136

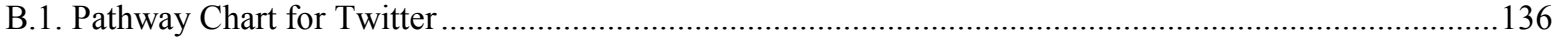

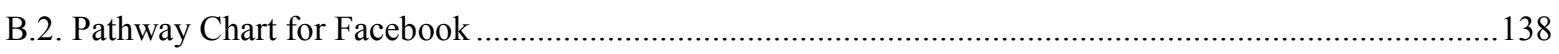

Appendix "C": Figures ..................................................................................................................... 143

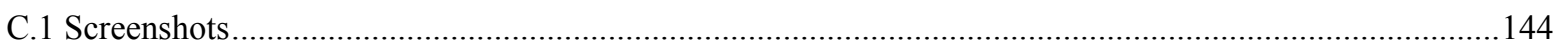

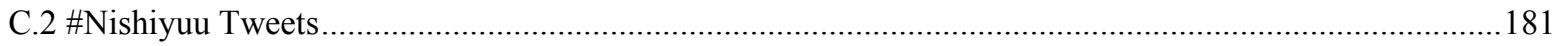

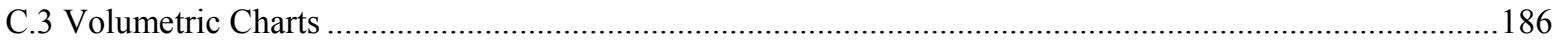

References (Figures) .......................................................................................................... 190

Bibliography ...................................................................................................................................... 194 


\section{List of Figures}

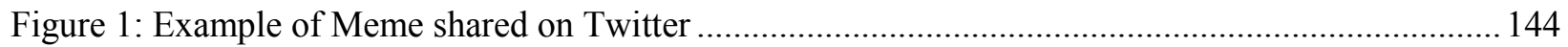

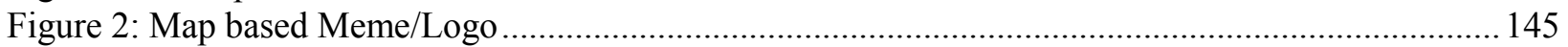

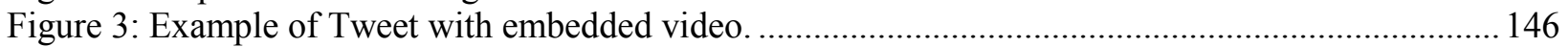

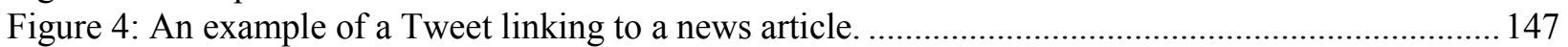

Figure 5: An example of a Tweet linking to a Flickr account. ......................................................... 148

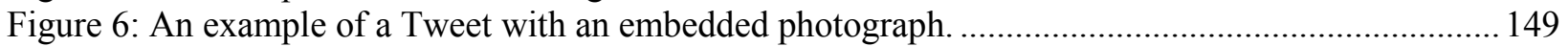

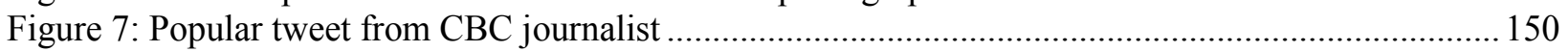

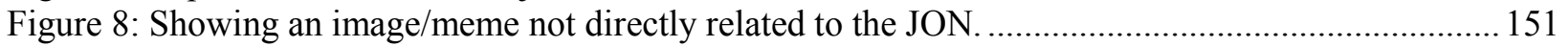

Figure 9: Image of Walkers during trek posted on Facebook Feb. 24th ................................................ 152

Figure 10: Example of a shared Facebook post with dialogue in comments. ........................................ 155

Figure 11: An example of Alternative Media link(s) on a Facebook post............................................ 156

Figure 12: Announcement of TV Interview, comments. ................................................................ 158

Figure 13: An example of a text-only post on JON Facebook group ............................................... 159

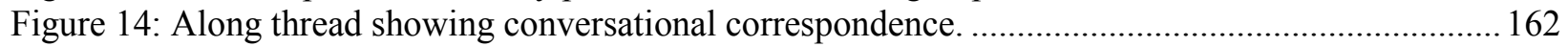

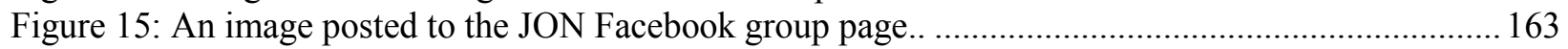

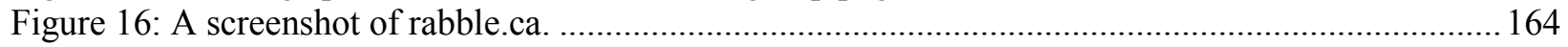

Figure 17: Comments section from Huffington Post article. ............................................................. 166

Figure 18: Conversation Thread remarking on media coverage .......................................................... 167

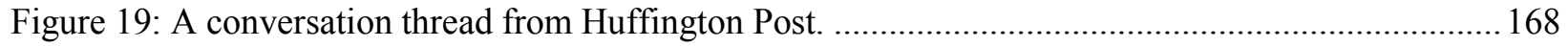

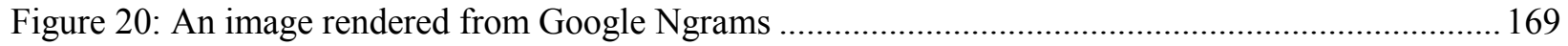

Figure 21: Map showing connectivity profiles of Aboriginal Communities in Quebec.......................... 170

Figure 22: Comments section from an Ottawa Citizen News Article................................................. 171

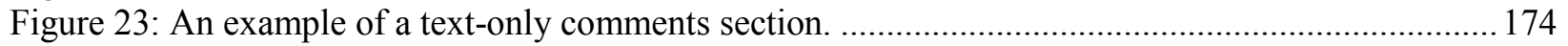

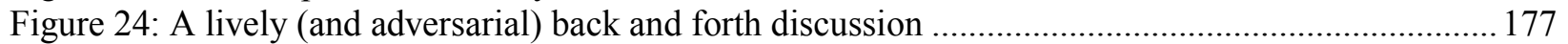

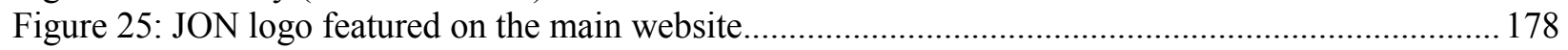

Figure 26: A tablet-drawn image comparing the JON to the Lord of the Rings Trilogy ........................ 179

Figure 27: Image of Google Earth map with points demarcating the JON trajectory.............................. 180

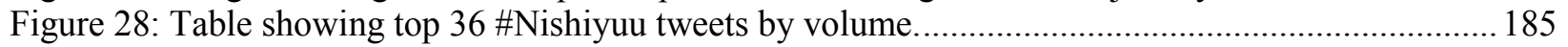

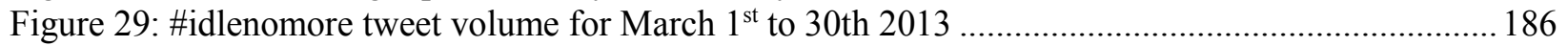

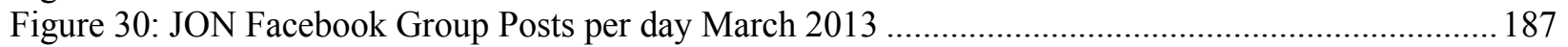

Figure 31: "Tweet volume of \#idlenomore hashtag adapted from Topsy. (Topsy, 2013)" ..................... 187

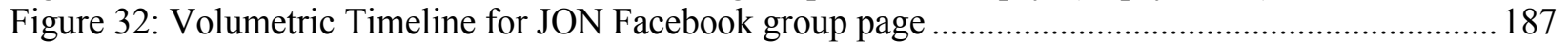

Figure 33: Data showing \#idlenomore volumes on arrival of Nishiyuu walkers (March $25^{\text {th }} 2013$ )........ 188

Figure 34: Data showing idlenomore traffic for the Week of 3-9 March 2013 ................................... 188

Figure 35: Data showing idlenomore traffic for the Week of 10-17 March 2013 .................................. 188

Figure 36: Data showing idlenomore traffic for the Week of 24-30 March 2013 ................................. 189

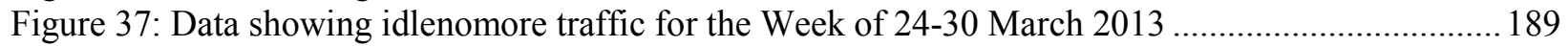




\section{List of Appendices}

Appendix "A" Network Map .......................................................................................... 133

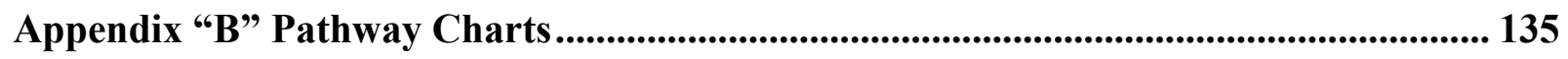

B.1 Pathway Chart for Twitter ........................................................................... 135

B.2 Pathway Chart for Facebook ............................................................................... 137

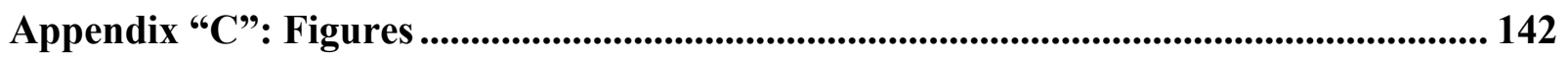

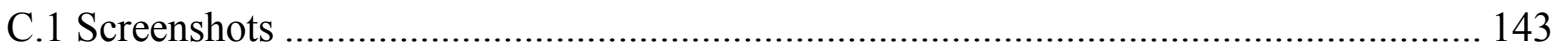

C.2 \#Nishiyuu Tweets ..................................................................................................... 180

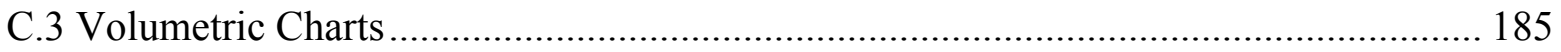




\section{Introduction}

\subsection{Overview of the Event(s)}

The Journey of Nishiyuu (JON) was a protest undertaken by several young people from Whapmagoostui, Québec. It began with seven youths who were inspired by the Idle No More campaign which itself had a large online presence. Their goal was to walk the upwards of 1,600km from their home in Whapmagoostui, Québec to Parliament Hill in Ottawa with hopes of meeting with the Prime Minister to address their concerns about their future. When they left Whapmagoostui on January $16^{\text {th, }} 2013$ there were seven walkers and one guide ${ }^{1}$. As they walked others joined the trek and when they arrived in Ottawa it was to a protest of around 5000 strong. During the course of their journey, they made an ever-growing impact on the digital landscape of social media, despite the early lack of impact in the mainstream media. By the time they reached Ottawa on March 25, 2013 the hashtag \#Nishiyuu was trending on Twitter in Canada ${ }^{2}$. The JON has its own website that was created while they were underway. Associated with the website are a Twitter account and a Facebook group. There are also several other online locations where conversations about the JON can be found on various social media sites, mainstream media sites, blogs, and other alternative media sites. Due to the nature of digital media, there is a record on these various locations of roughly how this conversation took shape and grew over the course of the JON. It is this record that will be analyzed for this project. With Social Media creating new public spaces online, there is a wealth of user broadcasted utterances with which to learn about digital communications media. The aim is to understand how the arc of the conversation developed, how the sites of communication relate to each other and their users, and what can be learned about the nature of digital communication through this kind of analysis. Ultimately this

${ }^{1}$ Nishiyuujourney.ca (n.d)

$2 @$ rabble.ca (March 25 2013) 
paper will use evidence from the conversation surrounding JON to advance the idea that digital communication is inherently iterative.

\subsection{Outline}

This project begins with a brief introduction to the case, and the methodology used in the analysis (Chapter 1). Next it provides a comprehensive review of the literature and theoretical perspectives that frame the analysis and provide the basis for the paper's hypothesis (Chapter 2). Following the literature review is an analysis of the JON conversation showing the iterative nature of digital communications (Chapter 3). The project concludes with an overview of the connection between the JON case and the theory (Chapter 4).

Chapter 2 provides a literature review and is divided into sections each addressing particular theoretical concerns. Section 2.1 deals with defining the terms for communication that will be used throughout the work. Hypertext is discussed showing that in order to curb user disorientation, the experiential level of online interaction is abstracted from its encoding. Next the changing meaning of media is discussed, overviewing how the terms 'media' and 'communication' have changed over time. This section shows that the experience of users differs from the architecture of design. It also highlights the importance of theory in understanding technology. Terms are shown to evolve with the technologies that they describe, but also to affect change in the design and understanding of technologies.

Section 2.2 discusses mediation beginning with a discussion of technological determinism. An attempt is made to find the middle ground between the effect of mediation on users and the effect of the societies from which technologies are formed on their formation. Further, the social impacts of digital communication are explored, both with relation to technology and society. Attached to this is a discussion of the increasing ubiquity of communication technologies. A 
theoretical perspective is provided suggesting that communications technologies are ubiquitous in the sense that they represent a fluid, but messy infrastructure. Through this section it is shown that there is a feedback loop of influence between society and technology.

Digital media and social media are the topic of Section 2.3 which deals with the dissolution of traditional boundaries resulting from the influx of digital communications technology. Firstly a distinction between public and private spheres is shown as ambiguous, merging, and having a potential for danger when users are unaware of the borders. Next the ease of reproduction and distribution is shown to have altered the understanding of mass media. It is suggested that it is not only size, but also time synchronicity that may affect these determinations. Thirdly the distinction between producers and consumers is shown to be blurred by the increasing importance of user generated content (UGC) creating more room for a middle ground. Overall this section deals with the convergence of previously separate fields, the transition from 'old' media forms to 'new' media forms, and the continuous negotiation of the boundaries within these domains.

Section 2.4 deals with the effects of affordances and communication technologies. Within this work the term 'affordance' will be used to describe that which is enabled by a technology as a meaning making tool ${ }^{3}$. A discussion of the transition to 'new media' shows examples of how form affects function. Following this, the design of interfaces is discussed, in particular those which take advantage of the constraints of digital communication. Examples of user

${ }^{3}$ Chandler and Munday 2011 define affordances as: "The different kinds of benefits and restrictions that a particular medium, tool, technology, or technique involves. James Gibson introduced the term affordances (which for him also implied constraints) in order to describe the interrelationship between an animal and its environment. Just as particular environment is conducive to certain kinds of life, so a particular medium is conducive to certain kinds of communication (due to its technical properties and the uses to which it is put): for example, the telephone affords simultaneous interpersonal communication at a distance, but constrains that communication to voice only." (Chandler and Munday 2011, 'Affordances.') 
determination are shown which reveal that adaptation can also create affordances. This section shows how design and use interrelate, showing that much can be learned from examining both aspect of digital communications.

Section 2.5 of the literature review deals with previous work in computer mediated communication (CMC). First is an overview of work that explains how users adapt to constraints and express emotions in text-based communication. Second is a discussion of the effects of anonymity in $\mathrm{CMC}$ and whether online spaces create a more impersonal environment or an opportunity for hyperpersonal interrelation. Third aspects of 'self' performance online are discussed showing that users presentations online are both fractured and converging. Ultimately what is gleaned from this section is that there are challenges to conventional understandings of communication presented by computer mediated communication, but ultimately that user adaptation will out.

In Section 2.6 the literature review is concluded with an overview of the specific theory behind the hypothesis that digital communication is inherently iterative. This section discusses pragmatic iteration as a tool for users to clarify their meaning, and gain greater insight into the meaning of others. Emphatic iteration is shown as an apparatus to stress, or infer the importance of information. The strategy of segmentation is described as involving the need for users to use multiple channels to manage the reception of information, both incoming and outgoing.

Following the literature review in Chapter 3 is an analysis of the JON case. This begins in Section 3.2 by outlining the conclusions provided by the visualizations undertaken by this project. The network graph shows the interconnections between locations within the conversation, and the affordances and media through which communications take place. The pathway analysis shows the experiential pathways of users navigating information within the 
conversation surrounding the JON. The volumetric timelines shows the shape of the JON conversation as it unfolded over time.

Section 3.3 uses the conversation surrounding the JON to outline the argument for iteration. It begins by providing evidence of the existence of iteration in digital communication using the conversation surrounding the JON. It then demonstrates that iteration is a necessary adaptation to digital communications, and ultimately shows that iteration is uniquely afforded by the digital medium. Section 3.4 explains the strategy of segmentation using the JON case. This is accomplished by showing why conversations take place across multiple platforms, why information or media can be found in different places, and that different interactions require different levels of synchronicity.

Finally, the work concludes in Chapter 4 by suggesting a kind of experiential text formation which would allow an understanding of iterative meaning making in Section 4.1. In Section 4.2 some future directions and opportunities for further study are discussed and the work concludes by tying the assumptions and conclusions back to the JON case in Section 4.3.

\subsection{Methods}

This section will outline the methods that will be used to analyze the online conversation. The main analysis will examine the information from the digital histories afforded by the various locations on the web where the conversations around the events took place. The utterances will be viewed as historical events, with an eye to the effect that their mediation and alterations in their display affect the view of them as such. This affects both their interpretation of historical events (i.e. in looking at them in their current form) and as current events in the time that they 
were posted (i.e. in extrapolating what they would have looked like at the time of posting) as suggested by Stone $2009^{4}$.

\subsubsection{Network Graphing}

In order to understand the scale and scope of the conversation the analysis will involve digital visualizations of the structure of the conversation. The first of these is a network graph, showing all of the locations, affordances and their interconnections. Locations within the digital conversation surrounding the JON are represented by square nodes, any affordances provided by these locations are represented by circular nodes. Each location node represents either an online location central to the conversation surrounding the JON (Facebook), or a related group of such locations (Misc. Alternative Media sites). The related groups were chosen to represent multiple online locations with similar key affordances that represent similar types of 'media'. For example, an alternative media site is the site offering conventionally formatted media from a source that would be considered outside the mainstream. These are often, though not exclusively, online only. A local newspaper may have a website, but would not be considered a mainstream media site. A more typical example is rabble.ca which provides crowdsourced news content, rather than commercially produced newsmedia ${ }^{5}$. A mainstream media site represents the online iteration of the conventional news media outlet (i.e. CBC, CTV, Ottawa Citizen, etc.). The grouping was done because affordances varied infrequently across similar sites (Media sites, specific pages within social media, and Blogs) and it would not have been possible to realistically encompass every location in which the conversation took place. Having fewer nodes made the chart easier to read and draw conclusions from. The graph shows the direct connections

\footnotetext{
${ }^{4}$ Stone 2009, 43-44;

${ }^{5}$ Rabble.ca (n.d) "About"
} 
between different locations, such as websites or social media sites, and the kinds of communications media that can be found/used in each 'location'. The arrows are colour coded to aid in visibility and while the rendered image is $2 \mathrm{D}$, the graph in the program is interactive. This makes it easier to see connections between objects in the chart, as you are able to drag a node, making obvious the connections. Each connection is represented by a directional arrow that describes the directionality available for linking. An arrow pointing from location 'A' to location ' $\mathrm{B}$ ' shows that it is possible to link directly from location 'A' to location ' $\mathrm{B}$ ' using a hyperlink, or transmissive media artifact. Manovich suggests that visualizing these direct connections shows how social media sites can act as a hub for many of the other locations in which the conversation takes place ${ }^{6}$. In retrospect it would have added to the analysis to use the arrows to represent whether media were available on site, or if viewing them required following a link. This could have been shown with the directional arrows. In the current chart all arrows point from affordances to sites so the arrows can aid in the visibility of the locations. The arrows were colour coded by location so when viewing the node representing an affordance, the colour of lines emanating from it shows sites on which the media can be found. Because of the directionality, the cluster of arrows surrounding the location node create a colourful display for the locations with the most media affordances. Orange arrows show links between locations, providing a visualization of which media are most linked to from other sites within the chart. As Choudury et al 2010 outline, many of the communications media exist on multiple sites, and this aspect of the graph shows similarities between the kinds of communications affordances of different sites ${ }^{7}$. Merely using the locations, the graph would show nothing more than is already known about the Internet; that it is an interconnected web. However, by adding the

\footnotetext{
${ }^{6}$ Manovitch 2010, 3-6

${ }^{7}$ See Choudury et al 2010, 4-5
} 
communication affordances that are shared amongst the different locations, the graph shows the transmissive properties between locations in light of the communication affordances that are provided by each site. The communications affordances are the means by which all linking takes place.

\subsubsection{Pathway Analysis}

The second type of visualization is a flow chart that shows the different pathways a user could navigate within different platforms (specifically social media, or websites) from a given entry point to any other location either within the original site, or to any accessible facet of the conversation on the Internet where further information on the subject could be found. These charts show the architecture of the possible experiences of a user upon 'entering' the JON conversation. Each chart begins with a list of entry points by which a user may find their way to a post and/or initial artifact (i.e. video, or news article). Working from left to right each chart shows the information, actions, or locations available to the user $^{8}$ following a particular path through the map. The chart is divided into sections by types of post/media artifact. The first section provides general pathways available for any post/media artifact on/in that location. For Example, the section covering 'Any Facebook post' contains pathways available for any type of Facebook post ${ }^{9}$. The subsequent sections for specific media only show the added pathways afforded by that particular media. Adding to those particular pathways are the general pathways that apply to all posts. These pathways can also be layered in the event of multimodal posts allowing users the option of following more paths for each additional modality.

\footnotetext{
${ }^{8}$ Provided the information, actions or locations are relevant to the JON, Tangential directions are noted, but not explored.

${ }^{9}$ Appendix B.2
} 
This kind of analysis shows the diversity of information available from any given starting point within the conversation in greater detail than the large network graph. It also provides insight into the layers of information available, and the many paths any given user may endeavour to go down. From a detailed look at any of these visualizations, we can draw conclusions about the effect of the affordance and interface of different websites and whether users are directed to different kinds and forms of information. It also shows how such architectures may realistically be circumvented by the actions of the user.

\subsubsection{Volumetric Timeline}

The third visualization is a volumetric timeline showing the size of the conversation, by number of utterances, on a given social media platform as it grows and contracts over time. This kind of visualization will follow Manovich's understanding of 'information visualization' ${ }^{10}$. Ideally this could be applied to multiple social media platforms and potentially even websites housing conventional media. Such a timeline has been rendered for individual posts in the Facebook group showing the volume of posts per day for the period of January $12^{\text {th }} 2013-J u n e$ $9^{\text {th }} 2013$ to provide a roughly even period of time before and after the protest on March $25^{\text {th }} 2013$ while encompassing the entire period of the walk. A similar chart showing the tweets for the hashtag \#idlenomore for the period from January 2013-April 2013 was used due to the inability to access the Twitter API due to the recent purchase of Topsy.com by Apple in early $2014^{11}$. Also used to compare was a chart rendered from data for the period of March $3^{\text {rd }}-30^{\text {th }} 2013^{12}$. This chart provides both the number of tweets and the number of sources tweeting per day.

\footnotetext{
${ }^{10}$ Manovitch 2010, 4-6

${ }^{11}$ Gregoriev 2013, See Appendix C.3 Figure 31

12 See Appendix C.3 Figure 29
} 
Because data was only available for March $1^{\text {st }}-30^{\text {th }} 2013$ for Twitter, a second Facebook graph was created only showing posts for March 2013 to allow for a direct comparison ${ }^{13}$.

\subsubsection{Characteristics of Affordances/Location Observations}

As part of the analysis of the JON conversation, it was important to look at particular media affordances that were available on multiple sites and compare them. Looking at specific utterances in the context of the characteristics of their location, interface affordances, and mediation will provide evidence of the impact of these factors. By looking at specific examples of user generated content and content posted by various media outlets, be they from mainstream or alternative media, this study will be able to compare how different platforms and forms of mediation affects similar kinds of content. For example, the number of responses to a particular post on Twitter may be less than a similar post on Facebook, but maybe shared or retweeted more. Also the kind of discussion that one would get on a mainstream media website differs from that of an alternative media website, or an embedded article on Facebook. There are examples of the same content (i.e. a photograph or a video) treated differently in different contexts on different platforms or locations with different affordances. This study will look at the formal aspects and context of related aspects of different posts or utterances within the JON conversation with reference to how they fit into the structure outlined by the analysis of the greater conversation. This will provide insight into not only the things that are being done specifically with the digital communications, but some of the effects of particular affordances or types of mediation. Throughout these observations I will refer back to the theoretical framework introduced in the literature review. As these observations provide evidence towards the hypothesis, they are expanded in section 3.3 and 3.4.

\footnotetext{
${ }^{13}$ See Appendix C.3 Figure 30
} 


\section{Literature Review}

In order to understand the nuances of digital communications, it is imperative to have a background of research from a wide variety of disciplines. The following will attempt to frame the understanding of digital communications by reference to literature produced attempting to understand communication, mediation, the effect of the interface, computer-mediated communication, and digital media. Sources referenced are those with long-standing impact in their fields of study, those that provide a unique understanding of the topic or those that have a particular relevance to the case of the JON conversation.

\subsection{Communication, to be Specific}

Communication is something that we do every day (even right now), but it is also something that is very hard to quantify. The definition of 'communication' in the Oxford Dictionary of Media and Communication contains 56 hyperlinks connecting to corresponding terms and subsections of the term which largely have complicated definitions of their own ${ }^{14}$. The word itself comes from the Latin communicare meaning 'to share" ${ }^{15}$. In its most simple definition communication involves "a process of interaction through messages or signals among or within humans, animals, machines, or plants"16. We can understand communication for this context as any attempt to transmit an idea, a message, or packet of information from user(s) to user(s).

Specifically within the umbrella of communication, this project will focus on digital communication which is the fusion of two related terms 'online communication' and 'computer mediated communication' (CMC). Online communication is defined as "any communication that

\footnotetext{
${ }^{14}$ Chandler and Munday (2011). "Communication."

${ }^{15}$ Ibid.

16 ibid.
} 
takes place over the Internet" ${ }^{\prime 17}$. The nearly synonymous 'computer-mediated communication' is defined as "any communication in which users interact via the direct use of a computer"18. Neither term entirely encompasses the scope of the digital communication surrounding JON. Occasionally there are instances of computerized content production that do not take place directly online. Also, asynchronous $\mathrm{CMC}$ does not require an active Internet connection and would not in some cases be considered 'online'. CMC is also inclusive of human-computer dialogues, which are independent of Internet access. Equally, there are forms of online communication that do not take place using computers (e.g. mobile phones) and occasionally not using them directly, as in the case of live streaming radio hosts. Imagine a Venn diagram of the terms 'computer mediated communication' and 'online communication'. If you were to draw a greater circle to encompass both terms, this will be what is referred to in this paper as 'digital communication' in order to incorporate the outliers in both cases. The act of either content creation or reception must occur either online or through computers to be considered digital communication, but it is not necessary that both conditions are met. While it may be the case that the definitions of 'online' and 'computer-mediated' are being renegotiated, the term digital communication will be sufficient to clearly define the boundaries of this inquiry. Also, as the conversation surrounding JON is largely about raising awareness for the walkers, much of it takes place in the public domain. This project will focus on communication that is both publically accessible (i.e. you or I can access it) and intentionally public (it has been published in a forum with no reasonable expectation of privacy). While this precludes private communications it does not banish them from thought as they can serve as potential entry points into the conversation.

\footnotetext{
${ }^{17}$ Chandler and Munday (2011). "Online Communication."

${ }^{18}$ Chandler and Munday (2011). "Computer-Mediated Communication."
} 


\subsubsection{Hypertext}

One of the difficulties in studying issues that traverse traditional disciplinary boundaries is that terminologies often overlap. A complication to understanding digital meaning making as text is the meaning of the word 'text' itself within the literature of communications technology. 'Text' commonly refers to the medium of 'textual' or 'text-based' communication ${ }^{19}$. Originally, 'text-based' communication had a straightforward meaning in computer mediated communication $^{20}$, but as the technology has developed it is necessary to divide it into categories; 'text-only' and 'text-based'. 'Text-only' communication; refers to communication constrained entirely to alphanumeric characters ${ }^{21}$. There are few forms of digital communication remaining that are constrained to text-only communication ${ }^{22}$. The second and more common form is 'textbased' communication; referring to communication centered around and primarily using alphanumeric characters, but allowing certain other affordances in addition ${ }^{23}$. For example a Twitter post would be considered text-based, as it is centered on alphanumeric characters, but it allows the use of images and other digital artifacts to be either linked or embedded into a post.

\footnotetext{
${ }^{19}$ I shall leave aside the obvious use of the word 'text' to refer to an SMS (text) message sent from a cellular phone. As this project does not include private communications of any kind cellular text messages will be overlooked, except to note that this is a further complication of the term 'text'.

${ }^{20}$ In fact it was the only option, early CMC platforms did not have any affordances beyond text. Early studies in CMC such as those done by Walther 1997 assumed the limitation of CMC to alphanumeric characters as read. ${ }^{21}$ Choudhury's description of a 'bag-of-words' is fitting for this definition, Choudhury et al. 2009, 333.

${ }^{22}$ Many online forums and mediated commenting sections remain text-only, unless they are using the infrastructure of a social media site. This is an interesting case where the choice of affordances affects the kinds of communication possible, but is clearly not something considered by the host site. A newspaper using Facebook commenting, for example, has no control over the affordances Facebook chooses to provide for comments. (CBC only allows text, whereas the Ottawa Citizen uses Facebook commenting)

${ }^{23}$ The obvious problem with this distinction between 'text-based' and 'text-only' is in the status of special characters and emoticons. This is not central to the understandings of this work, but it could be argued that where emoticons remain as typed, this request to 'text-only', and where emoticons are animated, this would constitute an added affordance and be considered 'text-based'. Also a hyperlink, presents itself visually as mere alphanumeric characters, however functionally it has a greater meaning and would fall under the category of 'text-based'. For a detailed discussion of emoticons in CMC see Jubril and Abdulla 2013
} 
It could be argued that there is a third form of textual communication in CMC. This would be where text ${ }^{24}$ is used to supplement a more dominant media. For example, a photo or video that has a caption would unlikely be considered 'text-based', but it still uses text to create meaning. The distinction between the second and third form of digital communication grows less important as affordances are provided for more kinds of mediated communication, with fewer constraints. However, the literature in computer mediated communication remains largely dependent on understanding CMC as 'text-based"25, so it is still important to understand why this distinction is made. Also, digital media are still examined based on their analog forebears. While the distinctions are muddied as the artifacts are digitized, they are still useful categories for academic understandings.

A further complication in the understanding of digital text is how to deal with the spatial dimension of hypertext. It is important to understand hypertext from its origins as it was created originally as a tool for navigating vast tracks of information. One of the stumbling blocks of this approach to interaction design is pointed out by Conklin who cites the propensity for users to get 'lost in hyperspace' ${ }^{26}$. This phenomenon existed due to the fact that, although the metaphor of space was apt, the disjointed, forking paths that were encouraged through the information was not a reproducible straight-line as had been previously experienced in other forms of written text.

The origins of hypertext are attributed to Vannevar Bush who envisioned a way of navigating vast tracks of information impossible to contain within an individual mind, too sizable to store in a single library, and increasingly difficult to reference ${ }^{27}$. Bush originally imagined what he called the memex which is possibly best described as an imaginary analog version of

\footnotetext{
24 alphanumeric characters

${ }^{25}$ See Hancock 2008; Walther 1997; Walther et al 2005

${ }^{26}$ Conklin 1987, 38

${ }^{27}$ Bush 1945, 37
} 
Wikipedia using miniaturized technology from the $1940 \mathrm{~s}^{28}$. Ted Nelson, in Computer Lib/Dream Machines expanded on the system applying it to the then modern computer technology of $1974^{29}$. What Nelson noticed was the potential of a system of linked reference that allowed information attached to words to be navigated through as experienced. This concept is the foundation of the internet as we know it today, although Nelson's original plan for the commercialized Xanadu was never enacted ${ }^{30}$.

Bolter suggests, that while hypertext allows for the same experience as print the traditional experience exists in parallel with a new form that allows the same referents to be experienced in a new way ${ }^{31}$. This is because "each topical sign is defined not only by the words it contains, but also by its relation to other topics. The computer makes these relations explicit and operative as a writer builds and the reader reads ${ }^{\prime 32}$. This provides a layer of interpretive meaning that did not exist in plain text, but that exists in the structure of connection ${ }^{33}$. Hypertext, for Bolter, is in a way the embodiment of intertextuality providing the illusion that these relations could even be mapped out ${ }^{34}$. However, since the hypertext, or the content to which it links is capable of constant and rapid change, the very mechanism that represents interrelation among ideas often serves to frustrate the hope of perfect interconnection as soon as it is created.

Hypertext is still the structural foundation of the Internet in the online world, but it is no longer the experience of it. Increasingly, users are abstracted from the mechanisms by which they navigate online, and this has a profound impact on their understanding of it. No longer is it the viewing direct referents of HTML, but representation layered atop coded signification.

\footnotetext{
${ }^{28}$ Bush 1945, 43-44

${ }^{29}$ Nelson 1974, 302

${ }^{30}$ Nelson 1974, 302; While Nelson's concept is foundational there remain key differences between www. and Xanadu (Conklin 1987, 23)

${ }^{31}$ Bolter 1991, 196

32 ibid.

${ }^{33}$ Bolter 1991, 201

${ }^{34}$ Bolter 1991, 202
} 
Experience of the internet relies on a metaphorical allusion to meaning. One example is the location metaphor which suggests that 'sites' are physical locations, connected to each other by 'links' which are proximate to either 'location'. Weiser describes how the location metaphor alters users' perception of the Internet: 'Technical people' understand the inner workings and hardware associated with the web so their conceptual model of the same is based on its infrastructure ${ }^{35}$. Most users, however, do not think the same way. Instead, "they think of the web in terms of sites and pages that belong to some person or organization... they don't think of [a webpage] as being on a server in some computer center, they think of it as being at somebody.com"136. As a result the geographic conceptual model of users is very different from the physical reality. This is especially important to understanding digital communication because naïve understandings of location as experienced by users, provide opportunity for interpretation. For example, the location of an image on a server provides less usable information than does its superficial location on a website and its endorsement by a named user.

These impressions have been further instantiated by the design of social media sites around the idea that despite the fact that the entirety of the user's data resides offsite on servers. The information that appears is on their page. The imagined infrastructure limits the potential for disorientation in hyperspace, as does the increased sophistication of web browsers that allow complex navigation through history and bookmarking tools. However, it remains within the definition of hypertext that it be always in flux, always changing. This means that while locations may be retread, this is not always possible with content. It is exceedingly unlikely for multiple users to experience the same path as one another. The appearance of information can be radically altered as if it were a static exhibit in a museum whose architecture was ever-

\footnotetext{
${ }^{35}$ Moggridge 2007, 463

${ }^{36}$ Moggridge 2007, 463
} 
changing ${ }^{37}$. Posts to heavily trafficked forums of any kind are notoriously hard to keep track of unless they are tethered to a personal account. Charlie Edwards notes that "while Twitter is very good at disseminating information, it is notoriously bad at making it persistent" ${ }^{38}$. Unless the user remembers exactly who tweeted certain information, or goes looking for it specifically through the search function, most content gets swept away as if by an electronic tide. The news feed is precisely that: new. As a result, online communication can be experientially ephemeral, but as Weiser noted, it is not necessarily technically ephemeral ${ }^{39}$. That is, while information once posted and passed over may seem to have disappeared into the din, it remains where it always was, on an actual server somewhere ${ }^{40}$.

\subsubsection{The Changing Meaning of Media}

While communication is fundamental, the purposes for communication have always been tethered to the technologies upon which they rely. Relatively recently, the conceptual understanding of these technologies as they pertain to the purposes of communication has been embodied by the concept of mediation. The idea is that the formulation of communication attempts are affected by the technology that affords them, and these technologies act as a middle ground between utterer and meaning. John Guillory attempted to trace the origin of the 'media' concept, suggesting that for centuries before the term came into common parlance, the concept was expressed by several theorists for whom the term was "absent but wanted" arts such as painting and music are now considered to be media. In addition, they are able to be

\footnotetext{
${ }^{37}$ This can be seen in the case of YouTube, which after being purchased by Google midway through last year altered some of its commenting affordances, but left posted videos and comments 'where' they were

${ }^{38}$ Edwards 2012, 223

39 "One wonders to what extent the premise of ephemeral information is correct in an age where the television and radio programs are no longer broadcasted "away" but instead accessible online for repeated individual perception"(Simanowski 2013, 195)

${ }^{40}$ The website topsy.com purports to have a record of every tweet since 2006

${ }^{41}$ Guillory 2010, 321
} 
remediated in digital media forms (JPEGs and MP3s), and were previously remediated in electronic media (Photography, Film, and Television) ${ }^{42}$. The concept of remediation is introduced by Marshall McLuhan in his 1964 Understanding Media: The Extensions of Man "the 'content' of any medium is always another medium.." ${ }^{43}$ Bolter and Grusin expanded on McLuhan's concept and adopted it for the digital age in their 1999 book Remediation: Understanding New Media ${ }^{44}$. However, Guillory contends that the idea of mediation makes media out of technologies that at their onset were not considered such even suggesting that it was theorizing mediation itself that made visible the existence of media ${ }^{45}$. The concept of mediation, therefore, was not made by the existence of media despite the term coming to us from Ancient Greece, by way of Aristotle's concept of mimesis ${ }^{46}$.

Guillory's historical tack provides an interesting insight into the revolutions of communications technology and their effect on theory. One historical account that Guillory outlines is that of Condorcet in 1795 who felt that the printing press undermined the rhetorical power of persuasive and powerful individuals by allowing a dissemination mechanism for $\operatorname{truth}^{47}$. This same argument has been applied to new media in much the same way. For example, Atton argues that digital technologies possess the power to overturn entrenched social and political norms ${ }^{48}$. As Guillory tracks the concept of media he proves that it is neither static nor 'new', but it is reimagined with new media. The word itself emerged in a crisis of novelty, but it

\footnotetext{
${ }^{42}$ Guillory 2010, 322.

${ }^{43}$ Guillory 2010, 322. Guillory quotes this from Marshall McLuhan Understanding Media: The Extensions of Man (1964; Corte Madera, Calif., 2003), p. 19

${ }^{44}$ Bolter and Grusin 1999 use McLuhan's term remediation to describe increasingly frequent act of taking an already mediated object like a newspaper article, photograph, or video and altering its form of mediation by 'remediating' it in digital form. For example, a video clip that aired as part of the news broadcast will often be rebroadcast digitally on the station's webpage. Whereas it was previously mediated by the television broadcast it was a segment of, when it becomes a digital artifact, its mediation is changed. (Guillory 2010,322)

${ }^{45}$ Guillory 2010, 322-324

${ }^{46} \mathrm{Ibid}, 322-323$, but then again there may have been a treatise or two in the library of Alexandria

47 ibid, 326

48 Atton 2003, 4-5
} 
solved as many past problems as it did future ones ${ }^{49}$. Another key point from Guillory's examination is that communication has not always been viewed as being for the same goals, nor as being always performed in the same way ${ }^{50}$. He suggests that there was a distinction between persuasion and communication. Persuasion was more insular and related to the verbal (rhetoric etc.), with the goal of convincing the listener to share the speaker's view. Communication was about sharing meaning and allowing others to understand the view(s) expressed. Early theorists relate this to print, but it could equally apply to new media ${ }^{51}$. Fundamentally Guillory argues that we had long been searching for a way to express what it was that got between our thoughts and our expression; the sender's meaning and the receiver's interpretation. The terminology we found was partially created by the more complicated media that it was invented to describe. If we keep this in mind when examining digital media, examinations of modern communication technologies may also provide new understanding of previous era technologies and methods. Guillory's perspective provides us with an opportunity for inquiry; if communication is not a static concept, what is it changing into now?

\subsection{Mediation}

When communication is mediated through technology, that mediation alters the form of meaning creation. Also, communication is inevitably affected by the technology that affords it. As the affordances of communication technologies change, so change the ways we create meaning.

Marshall McLuhan wrote at the cusp of the television age a revolutionary theory about the meaning and effect of media and mediation. He compared the creation of the medium of

\footnotetext{
${ }^{49}$ Guillory 2010, 322

${ }^{50}$ Guillory 2010, 327

${ }^{51}$ Guillory 2010, 322
} 
television to that of the invention of the printing press. His argument centered on the fact that communication technologies carry with them particular qualities by which they shape the kind of meaning that can be gleaned from any utterances they mediate. Further, he suggested that they also fundamentally alter the way a society is structured simply by their ontology. This is summed up by his most famous expression "the medium is the message" ${ }^{\text {". }}$. McLuhan sees the essence of communication as similar to language. He writes that "language is a metaphor in the

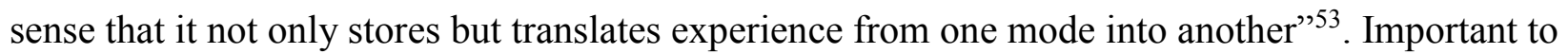
this understanding is the notion of translation which McLuhan compares to metaphor. He suggests it "is in our rational power to translate all of our senses into one another" ${ }^{54}$. McLuhan believed that television created a shift away from literate culture as being centered around the printed word towards a culture that was more auditory, relying instead on the spoken word. He writes; "the visual makes for the explicit, the uniform, the sequential, in painting, in poetry, in logic, history. The non-literate modes are implicit, simultaneous, and discontinuous, whether in the primitive past or the electronic present" ${ }^{35}$. McLuhan sees the change in mediation as fundamentally altering the way we process not only media, but all culture.

McLuhan staunchly believes that technology carries with it its own tendencies for human communication and action ${ }^{56}$. He goes so far as to suggest that the technology created by a society can even put the same society at a disadvantage. When referring to television, he suggests that literate societies are inept at dealing with the auditory domain inherent to televised communications ${ }^{57}$. However, McLuhan asserts that in understanding mediation we can overcome

\footnotetext{
52 McLuhan (1911-1980), 151

${ }^{53}$ McLuhan 1962, 13

${ }^{54}$ McLuhan 1962, 13

${ }^{55}$ McLuhan 1962, 73

${ }^{56}$ McLuhan 1962, 15

${ }^{57}$ McLuhan 1962, 37
} 
its effects ${ }^{58}$. This is perhaps one of the most important reasons people continue to come back to McLuhan, when understanding the impacts of new technologies. Regardless of how the impacts of mediation are created or understood, they exist and they profoundly affect our understanding and ability to iterate and interpret information.

While it is beneficial to study the adaptation of a new form of mediation, McLuhan suggests that it is the prolonged phase of adjustment to new technology, rather than the novelty of first exposure that is the true revolution ${ }^{59}$. This provides a compelling argument for why it is important to look not only at the potential theoretical properties of any medium as it is explored in its novelty; it is also essential to study how a new medium is actually put into use and how that use evolves. Understandings should focus not only on the inclusion of new affordances to a new technology, but also on the adaptation to the existing tools. If cinema theorists concluded in 1905 what the medium of cinema would be based on the novelty of the moving image, and the differences between the film reel and a photograph, it is obvious that this would not be a total picture of what cinema has become. As McLuhan would suggest, the revolution was pending; it was not waiting for a new affordance (although many were instituted e.g. sound, colour, CGI etc.), it was waiting for adaptation.

McLuhan's ideas were criticized by communications theorists and social theorists like Raymond Williams and others because of valid problems with the way that he described cultural endeavours as all-encompassing and left little room for individuality or performativity ${ }^{60}$. However, with the radical changes of new media several aspects of his theory bear reconsideration as they pertain to these new media. Paglia writes; "Some theorists tried to shore

\footnotetext{
${ }^{58}$ McLuhan 1962, 15

${ }^{59}$ McLuhan 1962, 33

${ }^{60}$ Paglia 2003, 268, Freedman 2002, and probably because he said things like: "the training of muscular sensibility among girls seems to favor the more delicate muscular coordination consistent with reading the printed word." (McLuhan 1978, 261)
} 
up their system by redefining the Internet as a postmodern medium, but the idea was misconceived. The fragmentation or discontinuity they thought they saw was really the proliferation, multiplicity, and rapid pulses of electronic media that McLuhan correctly interpreted in physiological and neurological terms. Surfing the web (significantly, a naturesports metaphor) means catching its wave motion and feeling its tides and eddies. The web has weather, particularly when news events unleashed storms of popular sentiment. Although the body is quiet and dormant at the computer, the web works by sensory overload, through which the server navigates along a zigzag track like a sailboat tacking at sea" ${ }^{\prime 61}$. Her connection to McLuhan is not unique. Alan Kay, a pioneer in human computer interaction, was also heavily influenced by McLuhan and the idea that the age of computers will further reshape human consciousness ${ }^{62}$."What McLuhan was saying is that if the personal computer is truly a new medium, then the very use of it will actually change the thought patterns of an entire civilization"63. Arguably our understanding of mediation and previous media will involve a complex layering of effects and countermeasures when incorporating any new media into our lives.

Raymond Williams offers a counter point to McLuhan's 'medium-as-message' mantra by suggesting that technology is created and used by people who build in, and filter through their social biases and it cannot be separated from these factors. In his 1977 work Technology and Cultural Form he puts forward four factors he see as central to his more complicated understanding of mediation:

(1) the technological character of individual media, (2) the political and economic structure which influences the research and development of new technologies and

\footnotetext{
${ }^{61}$ Paglia 2003, 268

${ }^{62}$ Moggridge 2007, 159

${ }^{63}$ Moggridge quotes this from Kay, Alan "User Interface: a Personal View" in The Art of Human Computer Interface Design edited by Brenda Laurel (Reading, MA: Addison-Wesley, 1990) 1993
} 
affects their growth and institutionalization, (3) the cultural assumptions concerning the appropriate use of the media which affect both producers and consumers, and finally (4) the actual distribution and "flow" of content within the media."64

While it seems obvious to state that technology is created for a purpose, that purpose also determines how it is shaped. Thus technology is shaped by and in turn shapes its users. Control over the instruments of mediation is central to any work in $\mathrm{HCI}$; as the interface shapes its users, so it is shaped by its designers to align with any alterations. Freedman 2002 suggests that Williams would describe the internet as neither empowering nor decentralizing ${ }^{65}$. He cites strong evidence that flow of traffic online funnels through gatekeeping sites, which echo flows of resources in the 'real' world ${ }^{66}$. Williams' critique of McLuhan is not that there is no effect of media, but rather that mediation is not all-powerful. He also noted that due to the social nature of mediation, McLuhan would require reimagining as situations change and technologies turn over $^{67}$. It is clear from the shape of Digital media today that it reflects the social forces involved in creating it, but by no means absolutely. Monetization is a key aspect of the modern web, but so are counter-intuitive ideas like open-source software and Wikipedia level crowdsourcing projects. Williams rightly advocated that social forces should be seen as a driver as driver. However, if the media was created by a society which in turn was partially created by its relation to previous media, causation becomes a tenuous path.

\subsubsection{Is Media Determined?}

From McLuhan we get a sense of the importance of the medium in meaning making, but others argue that any impact of mediation is a mirroring of the social factors inherent to the

\footnotetext{
${ }^{64}$ Neuman 1974, 502

${ }^{65}$ Freedman 2002, 439

66 Ibid, 437

${ }^{67}$ Freedman 2002, 436
} 
people for and by whom it was created. Charles Ess grapples with this dichotomy from a philosophical perspective, contrasting technological determinism; which would claim that "CMC technologies are not value neutral but rather embed and foster the cultural values and communicative preferences of their designers" with technological instrumentalism; which argues that 'these technologies cannot simply overrun 'target' cultures and homogenize them into a (Western) cultural monolith: by themselves, CMC technologies are not sufficient for an inexorable process of democratization - or, more broadly, computer mediated colonization" ${ }^{68}$. On the one hand was the convincing argument that producers and designers of communications media and code, quite literally, encode their own communications preferences and biases into their interface. This interface, then has an indelible effect when used which further instantiates the encoded preferences and biases. On the other hand, there is substantial evidence that the mediation itself cannot override an individual's ability to manipulate a tool to be wielded for their own ends ${ }^{69}$. It is clear that both claims have merit, so to find the middle ground between these two extremes Ess argues for a kind of soft determinism as proposed by Don Ihde (1973) ${ }^{70}$. Ess takes advantage of the dichotomy central to CMC to "support a move beyond the Cartesian dualism to more holistic metaphysics and epistemologies"71. For the purposes of computer mediated communication it is important to note that, while HCI practitioners aim to eliminate interference from the medium, this will remain impossible. Also, theories of mediation will always be open to deconstruction by changing patterns of use. So, if we are never to eliminate the medium, nor fully encapsulate it, what remains? What remains, is to describe the mediation as it exists at a given time, make comparisons between these descriptions and past descriptions to

\footnotetext{
${ }^{68}$ Ess 2002, 227

${ }^{69}$ Ess 2002, 219

${ }^{70}$ Ess 2002, 227

${ }^{71}$ Ess 2002, 235
} 
analyze evolution and adaptation; and to bear all of this in mind while creating interfaces that allow for an attempt at minimizing structural bias, when it interferes with use. In other words an iterative process of research, design, and theory ${ }^{72}$.

Jan Fernback 2003 suggests that the kind of communication expressed in CMC is something beyond conventional textual communication. Fernback observes that " $\mathrm{CMC}$ requires more nuance than simply typifying online discourse in terms of either oral or literate culture. Clearly, CMC displays elements of oral as well as textual communication." ${ }^{, 73}$ Fernback's observations muddy the distinction of text ${ }^{74}$ "as mediated human communication becomes more and more non-linear, decentralized, and rooted in multimedia, the distinction between orality and literacy becomes less evident and less important" ${ }^{\text {"75 }}$. Fernback relies on Finnegan 1988 who first suggested that there need not be a dichotomy between literacy and orality as is suggested by theorists like McLuhan. Further, she suggested that the advent of a new communications technology allows for a space to rethink old assumptions ${ }^{76}$. Where McLuhan describes the transition of the Renaissance as "the frontier between two cultures and between conflicting technologies" ${ }^{\text {"7 }}$, perhaps this is not representative of the transition to new media. There is compelling evidence that what was 'conflict' in the case of the printing press and electronic media, exists in the transition to digital media as convergence.

If Fernback is correct, this means that in examining $\mathrm{CMC}$ we are no longer looking at an isolatable text-only interaction (even in an instance where alphanumeric characters are the only affordance available), but one that is infused with experience and culture. The multimodality

\footnotetext{
${ }^{72}$ The iterative design process is loosely defined as a "design-test-modify [repeat] interface development cycle" Waterworth 1992, 39

${ }^{73}$ Fernback 2003, 30

${ }^{74}$ Referring to the $\mathrm{CMC}$ understanding of text as strings alphanumeric characters; literal written words

${ }^{75}$ Fernback 2003, 43

${ }^{76}$ Fernbak 2003, 43 (Fernback cites Finnegan 1988:175)

${ }^{77}$ McLuhan 1962, 172-173
} 
means that rather than looking at how text-only or text-based communication works, we now need to translate mediation from several different forms of communication that are merging together in more complicated ways. Fernback's understanding of digital text as an oral culture fundamentally alters how we conceive of text-based CMC. It may also suggest that text based chat is closer to a telephone call, than to the written word. This manifests in the ease of transmitting information, which can often supersede the impulse to filter. Fernback suggests that "even without sufficient information to discredit the story, people often retell through email forwards urban legends that they suspect might be false to 'cover the bases' in case the story is true. The legend may not be credible enough to transmit to someone face-to-face, but the immediacy and ephemera rally of CMC allows the user to distribute the legend and participate in the ritual of storytelling without much effort." 78 This impulse to participate through sharing is enabled on many social media websites today. The influx of this kind of information may serve to harm the aims of the JON if users have become skeptical of news not reiterated through conventional news channels. The simplicity of reiterating stories in the digital world can sometimes even muddy the distinction between the important and the trivial.

From McLuhan we get the importance of understanding that communication is technologically mediated and that mediation has an effect on interpretation. From Williams we temper the determinism inherent in McLuhan by remembering that technologies are created and used by social beings and that influence will be built into the systems and occasionally drive their creation. The critics are polar opposites, as Ess describes, but the ideas are not necessarily bound for annihilation. A combination of the two approaches gives us a circular pattern of critique beginning with tools that alter our consciousness towards the creation of more similar tools, or beginning with a consciousness that is wired to build particular tools which evolve as

\footnotetext{
${ }^{78}$ Fernback 2003, 42
} 
does our socialization and use of them. It is important to bear in mind both the effect of mediation and the social factors of creation and use of technology to understand how we create meaning in any format. Functionally, these 'struggles' are going on every time we interact online. Whether the interface is imbued with a social bias or simply has an agenda that is a function of the medium itself, there is still an impact on how we will use and process the information it holds. Hansen et al. 2011 provide a view of the middle ground. Rather than technologically deterministic, the authors suggest that "technologies affect the material world which in turn affects the social world; an undeniable fact" ${ }^{39}$. The simple fact that a counter cultural protest can make use of tools created very much in the mainstream proves that despite the interference of mediation, the primary purpose is to mediate (that is, to facilitate to the flow of information). Still, one of the laudable traits of the internet is its potential for democratization, or at least globalization. Ultimately the scale of the end product of the digital tool and also its users is larger than any previously existing communications technology. If this is not the case now, it will be soon. The population of the world is as large as it has ever been. Not everyone has access to the Internet, but the potential exists for a time when they would. This is simply not a possibility for any previous form of mediation due to the difficulty of reproduction and distribution inherent with immutable physical objects. True, access to a piece of paper is more prolific than access to a smartphone, but the volume and reach of utterances would be stunted in comparison. It is the potential for so many users to be connected and able to share ideas that is revolutionary.

\footnotetext{
${ }^{79}$ Hansen et al. 2011, 12
} 


\subsubsection{Social Media and Social Movements}

Communications theory was fodder for those looking to argue complex issues of control over information as a tool for social and political domination or upheaval. This perspective colours some of the important criticisms of McLuhanism that suggests that the tendencies of mediation are structured by the societies that create them. Williams, for example argues that the goals, mechanisms and workforce all encode their social programming into technologies ${ }^{80}$. In turn users make use of the programs in ways ascribed to them by society ${ }^{81}$. The social critique thus turns into a rhetorical device to prove the instantiation of particular ideologies into all aspects of culture, and the means by which they are spread. In doing so, they provide good reasoning to suggest that the creation and use of technologies are socially driven, but focus perhaps too heavily on mass communications technology, and its conventional use. One of the revolutions allowed by digital technology, is that tools previously only available to a select few who had the resources to produce and disseminate mass communications are available to more people. This availability has been heralded as a democratizing aspect of digital media ${ }^{82}$. However, there is still a great deal of evidence suggesting that the failure of this potential to actualize proves in part the arguments of those who suggested the social construction of communications media as unavoidable. In theory, the digital world has the capacity to overwrite societal norms, but in reality the world online still resembles the rest of the world ${ }^{83}$. Internet sites have largely found their way into the capitalist system, finding ways to make money and provide business models like any other company. While there are business models that have revolutionized understanding of media, they are so driven by profit like any other business. Traffic on the Internet is hierarchically structured just as access to information was structured

\footnotetext{
${ }^{80}$ Neuman 1974,502

${ }^{81}$ An aspect of this can be seen in SIP theory; Hancock et al 2008, 285

${ }^{82}$ See Atton 2003 4-5

${ }^{83}$ Freedman 2002, 438
} 
previous to the Internet. Freedman provides evidence that the Internet represents another winner takes all market, with a large majority of users funneling through the highest profile, and highest traffic sites ${ }^{84}$.

Atton 2003 argues that the primary goal of activists using media, remains political and that media activism includes not only providing alternative information but also needs "to envisage a media formation that corresponds with the non-hierarchical, local-global framework of the movement it supports" ${ }^{\prime 85}$. Failing complete media upheaval, activists should at least attempt to rebalance media accounts with media centered on protest. This is not what we see from the JON. Instead the JON participants use the tools at hand, despite the fact they may have inbuilt hierarchies and power structures that they, in part, are trying to subvert. Any rebalancing is necessarily channeled through social media sites that do not fit Atton's criteria ${ }^{86}$. Atton's criticism is valid but it underestimates ${ }^{87}$ the complexity of the systems involved. Limiting media use to sites devoid of the hierarchies and biases that one is fighting against, may serve only to further instantiate the disadvantaged status one is trying to overcome. Simply, the complexity of a program like Facebook and its communications affordances are not within the realm of possibility for a small organization to recreate. We are beholden to social media platforms in the same way as we are to languages. If we wish to be heard and understood the format of our speech must be open and available to the widest audience, even if that utterance is thought by a JON protester to carry with it centuries of colonial baggage. At the same time it is important to reflect on these contradictions between form and content, as they hold important revelations as to the impact of design considerations. For example user studies are designed primarily to cater to

\footnotetext{
${ }^{84}$ In the year 2000 the top 0.1 percent of sites attracted a third of all user volume, and the 83 percent of all hits were drawn by the top 10 percent of sites. Freedman 2002, 438

${ }^{85}$ Atton 2003, 12

${ }^{86}$ Atton 2003, 5, 12

${ }^{87}$ underestimates me not be the best word here, it may be better to say "idealizes the ability to counter"
} 
the average user and ensure the highest number of satisfied users. This is especially the case for communications media as these have both the broadest user base and the least concrete understanding of specific user preferences. Catering to a generalized user profile can put minority groups unintentionally at a disadvantage, mainly as an extension of existing discriminatory conditions.

This phenomenon has been noticed with Twitter in an examination of the power relations within academics studying digital humanities (DH). Edwards 2012 writes: "The lack of mutuality in Twitter relationships can simply replicate or 'reify' the off-line hierarchies of DH. In practice, this means that the net here is not neutral but biased in favor of those with more and better connections. Their messages are the most likely to be distributed, their voices the most likely to be amplified" 88 . This effect can be mitigated by the use of hashtags which partially democratizes users tweeting on a particular topic, as can the principle of reciprocal following, but Edwards claims that "the bias is still there, and disproportionately and daily inhibits the reach of new users and those at the margins of the $\mathrm{DH}$ network who have limited access to its more powerful modes. ${ }^{\prime 89}$ The same power structure exists with any Conversation in the Twitter-verse. In the case of the Journey of Nishiyuu, where power relations within the group are still being negotiated and established, the more important implication of these power relations are in those that pre-existed their movement. These can be seen in the more influential users' posts being retweeted more frequently than users who may be influential in the movement, but not established within Twitter. For example, a tweet from@jianghomeshi (a verified account associated with the established CBC journalist Jian Ghomeshi) reading "The \#Nishiyuu walkers are a true inspiration. And deserve more attention than visiting pandas." (2013-03-25 3:15:00

\footnotetext{
${ }^{88}$ Edwards 2012, 222
}

${ }^{89}$ Edwards 2012, 222 
PM) ${ }^{90}$ received 357 retweets and 102 favourites, whereas a Tweet from @Nishiyuu (the Twitter account associated with the official JON website) posted less than 20 minutes afterwards (201303-24 3:38:00 PM), received only 60 retweets and 16 favourites. ${ }^{91}$ A post an hour and a half earlier with similar content ${ }^{92}$ from a relatively less influential (and more average) Twitter user 'Jessica M' (@marymacjess) received only four retweets and two favourites. ${ }^{93}$ Of the top 25 most trafficked posts ${ }^{94}$ for the hashtag \#Nishiyuu only six were posted by users who had not selfidentified in their Twitter profile as either politicians, political groups, journalists or media outlets. Among those posts two were from the @Nishiiyuu account ${ }^{95}$.

As a counterpoint to the concern about power relations transporting themselves online, there are several examples of social media being used during the Arab Spring. For example, social media can be used to build and grow social movements that aim to overthrow those in power. Particularly, the ability to access social media on mobile phones allows for a sophisticated tool in burgeoning social movements that involve real world interaction as well as coordinated information communication ${ }^{96}$. Lim 2012 suggests "social media may be viewed both as technology and space for expanding and sustaining the networks upon which social movements depend"97. Twitter was seen as highly influential in its ability to inform the outside world of alternative views of the facts on the ground and alert them to the crisis in Tahir Square

\footnotetext{
$90 @$ jianghomeshi (March 25 2013)

$91 @$ Nishiiyuu (March 25 2013)

92 "Pandas are cool and all, but aren't there more important things to pay attention to, Mr. Harper? Like \#Nishiyuu perhaps?”@MaryMacJess (March 25 2013)

$93 @$ MaryMacJess (March 25 2013)

94 \#Nishiyuu (November 11 2013)

${ }^{95}$ There was one post that represented a series of posts surrounding the same article by the Huffington Post, but as the volume of posts was measured by the reference to the article, and not to any of the individual posts this was considered as a post by a media outlet. See Appendix C.2 Figure 28

${ }^{96} \operatorname{Lim} 2012,236$

${ }^{97} \operatorname{Lim} 2012,234$
} 
in $2011^{98}$. Lim provides the example of 'Esraa the Facebook girl' to show how imagery can be spread easily using social media and can actually create a corresponding social movement ${ }^{99}$. It was suggested that many of its users joined the April $6^{\text {th }}$ Youth Movement's Facebook group not necessarily for affinity with the movement, but because it was seen as trendy after the arrest of the group's leader ${ }^{100}$. Importantly, she does take note of the fact that the social media aspect of the uprising was only a symptom and acted as the facilitator of pre-existing conditions for social change. That said, it is a powerful facilitator. Wael Ghonim, an online activist central to the protest "called the Egypt uprising 'Revolution 2.0" referring to the central role of Facebook in the protest suggesting that Facebook founder deserved thanks ${ }^{101}$. Because of the complexity of the issues involved no single example will provide the victor between the techno-utopian scholars who believe the Internet to be the saviour of freedom, or the techno-dystopian scholars who believe that its power is precisely what makes it a threat to freedom as the powerful wield it to further their own ends ${ }^{102}$. It is likely that there is a multiplicity of truths created by each individual circumstance. It is also clear that the nature of these technologies is evolving rapidly, so truth will be increasingly hard to pin down.

\subsubsection{Ubiquity}

One of the specific traits of digital media today is its portability. The metaphor of plugging in and out has long been in the lexicon of digital commenters, but recently the idea of ubiquity has been equally parried about. The idea that technology is everywhere and all around is easily claimed in a world where so many people carry in their pockets devices with more computing power than that taken on Apollo 11's moon landing. The idea of ubiquitous

\footnotetext{
${ }^{98} \operatorname{Lim} 2012,243-244$

${ }^{99} \operatorname{Lim} 2012,240$

${ }^{100}$ Ibid.

${ }^{101} \operatorname{Lim} 2012,231$

102 See Lim 2012, 232 for some of the scholars weighing in on either side of the debate.
} 
computing has a specific understanding; seamlessly integrating into our daily lives ${ }^{103}$. Dourish and Bell 2011 outline some early understandings of ubiquitous or near ubiquitous computing. One of their first examples is from Mark Weiser who suggested in the 90s a forward thinking move away from the desktop metaphor. "In [Weiser's] approach, the computational experience moved away from the desktop and into the everyday world and was distributed across a range of devices each specialized to particular sorts of tasks." ${ }^{104}$. While Weiser's approach required more devices, it can be seen as synonymous with the kind of mobility we have with our devices today. We still rely on powerful stationary computers, but have devices with varying portability that make concessions in functionality for size. Synonymous though it may be, the current model still falls short of Weiser's total integration of technology into daily life. Despite the progress being made in the miniaturization of computing technology, the across-the-board progress of computing technology suggests that we will be in this paradigm for some time for as the capabilities of our mobile phones and tablets increase so too does the capability and capacity of our desktop computers and laptops. Also, the trade-off between portability and screen size is at an impasse until high-resolution flexible devices, portable projectors, or head-mounted displays become viable alternatives. For Weiser, the end of computing is quite literally its end. The technologies should "weave themselves into the fabric of everyday life until they are indistinguishable from it" ${ }^{105}$. However, this seems to conflict with the idea that computing today could be considered as having achieved this end.

Dourish and Bell suggests an alternative to viewing disappearance as literal invisibility, and instead suggest that it is not about what is there, but what is noticed ${ }^{106}$. To make this

\footnotetext{
${ }^{103}$ Dourish and Bell 2011, 95

${ }^{104}$ Dourish and Bell 2011, 11

${ }^{105}$ Dourish and Bell 2011, 95; the authors quote this from Weiser 1991, 94

${ }^{106}$ Doursih and Bell2011, 95
} 
understanding work they rely on the understanding of infrastructure proposed by Susan Leigh Star as a 'relational concept ${ }^{\text {' }}{ }^{107}$. Here, an infrastructure is a figurative 'everywhere'; perceived differently based on its use, and the perspective of the user. They use the analogy of the sewer system which to most of its users is a uniform and unchanging system that is ubiquitously available. This experience is fundamentally changed when experienced differently. For example: "a city engineer [is] exposed to the daily practicalities and pragmatics of wastewater management and treatment, [so] the sewer is not an infrastructure but rather a site of work, and not uniform but instead highly localized and variable"108. An important tenant of this claim is that these are relationships to the infrastructure which need to be steadily maintained and reflexive. The sewer is no longer ubiquitous to the user who finds it backing up into their house. Importantly, this infrastructure "is always unevenly distributed, always messy"109 and an infrastructure that is otherwise could never be realized ${ }^{110}$.

By aligning ubiquity with infrastructure, Dourish and Bell achieve two things. The first is that they create a way of understanding ubiquitous computing not as an ideal, but as a practical and tangible reality. The second is they debunk the notion that ubicomp will ever be the former. They go on to stress the importance of studying it as it is rather than how it is imagined ${ }^{111}$. For the purposes of this project it will be important both to understand how access to digital communication affects its use, and to attempt to understand the current reality of digital communication as a kind of infrastructure that is ubiquitously available except for when it is not. New technology has allowed computation to break out of the traditional spaces it once inhabited.

\footnotetext{
${ }^{107}$ Dourish and Bell2011, 26

${ }^{108}$ Dourish and Bell 2011, 26

${ }^{109}$ Dourish and Bell 2011, 27

110 Dourish and Bell 2011, 28-29

${ }^{111}$ Dourish and Bell 2011, 31
} 
This causes us to understand space in radically different ways ${ }^{112}$. It creates the ability for people to experience space both physically (where they are) and digitally ('where' they are interacting). Individuals 'live tweeting' an event provide a good example of this kind of layering ${ }^{113}$. Understanding our interaction with the devices that provide our access to the digital world in this way helps us to understand that they are the infrastructure by which any affordance and mediation are enabled and, like the sewer system, they are largely unnoticed by users unless something overflows. For designers and HCI practitioners, the infrastructure is a site of work and many important decisions are made based on these factors. Like the city engineer, the aim is to keep the infrastructure from identifying itself to the user. However, given the profound impact of mediation on meaning, achieving this goal means that users will largely be unaware of the inherent interference. For the JON it is all well and good to say that cell phones with internet access provided access to the event in an amazing new way, but the information still had to travel through a constrained and complicated infrastructure. Yes we have attained a degree of Weiser's disappearing interface, but the interface is still a powerful mediator of meaning. Another important aspect of ubicomp is that it implicates other technologies into the digital world. Users are choosing their platform not due to access to a computer, but privacy and distribution networks. This means that they make choices between digital and non-digital communications (To Facebook chat or SMS for example) based more and more on affordance rather than access.

\subsection{Digital Media and Social Media}

There has been ample research done pertaining to the transition from print and tradition media to online journalism. It is also a topic of much discussion in the media itself, which provides introspective views, usually on the pitfalls and apocalyptic eventualities of the

\footnotetext{
${ }^{112}$ Dourish and Bell 2011, 108

$113 @$ onthebeat1 and @ryanpaulgibson (March 25 2013); The live tweet from the CBC for the Journey of Nishiyuu can be found here: http://live.cbc.ca/Event/Journey_of_Nishiyuu_comes_to_an_end
} 
transition. This section will provide an introduction to some of the issues that are most relevant to the JON case. Traditional media websites provide a locus for the JON conversation, as well as being a platform for mass communication themselves. Also, the media objects (exemplary of digital media) can be ported or linked to other key locations. As such, it is important to understand how the affordances provided with newsmedia online came to be shaped as it is, and how interactions with these media objects can affect their consumption.

\subsubsection{Blurring Lines: Public and Private}

With new media come new locations to communicate and along with them a muddled sense of public and private spheres. Legally, the lines between public (shared) communications and private (hidden) communications must be imparted to the user, but this is often done in the rarely considered terms and conditions sections that are a precursor to account creation on most sites. Notably, Facebook privacy settings are updated and users need to reset them as they have occasionally defaulted to a lower setting after an update of the site. As a result many users are unaware of who can access their sites and pages, access their data, and interact with their media. Zizi Papacharissi suggests that the spheres of private and public, as well as online and offline, have become entangled as SNSs (Social Networking Sites) adopt more and more of the affordances of traditional communication media ${ }^{114}$. Affordances that aim to make communication easier are being provided in venues where synchronous dyadic conversations are not always socially appropriate. Users of social media have likely witnessed a disagreement in the comments section that would have been more appropriate in a private message. At the same time an engaging interchange will also encourage the participation of others in the conversation

\footnotetext{
${ }^{114}$ Zizi Papacharissi 2012, 208
} 
which suggests there is a place for this affordance in a public setting ${ }^{115}$. Landert and Jucker write of the complications that the digital environment creates regarding this segmentation between the public and the private sphere. They argue that conceptions of public and private are merging ${ }^{116}$. They also suggest that there are different levels and understandings of 'public' that scale outwards based on perceived levels of accessibility ${ }^{117}$. The analysis of the JON for this project stays within the public sphere, using only information accessed in a space where there is no reasonable expectation of privacy (most of the communications were aimed expressly at raising awareness of the JON or its ideals), but it is important to note that there is a level of ambiguity. Also, despite the fact that their utterances are publically available, many users may rely naively on the anonymity of volume ${ }^{118}$.

Biscuitwala et al. 2013 outline the dangers of the ambiguities of public and private spheres online. They express concerns about the safety of citizen journalists, who present both an opportunity and a problem for new media. While contributors may assume they are anonymous, the authors suggest that "even in the U.S. content contributors are at risk of being physically tracked through their digital communications" ${ }^{\prime 19}$. This is problematic because many do not consider social media as something that is high-risk ${ }^{120}$. The authors maintain that "the [] vulnerabilities are artifacts of the current architecture of networked communication and publishing platforms, including social media" ${ }^{121}$. Part of the problem is due to the fact that

\footnotetext{
115 Choudhury et al 2009, 332

${ }^{116}$ Landert and Jucker 2011, 1422

${ }^{117}$ Landert and Jucker 2011, 1423-1424

${ }^{118}$ In viewing the JON public Facebook group posts I saw several personal photographs that had little to no relevance to the walk itself, but instead to the community surrounding it. People conversed about their personal life regularly, either unaware of the accessibility of their posts or unconcerned by it.

${ }^{119}$ Biscuitwala et al. 2013, 850

${ }^{120}$ Biscuitwala et al. 2013, 849

${ }^{121}$ Biscuitwala et al. 2013, 850; The ominous text message sent to protesters in Kiev recently spoke to the precariousness of ubiquitous technology by claiming to log protestors based on the locations of their cell phones (Walker and Grytsenko; Jan 21, 2014)
} 
information is stored primarily out of the user's control on servers that can potentially be accessed by governments despite expectations of privacy ${ }^{122}$. Access can also be disrupted as accounts and sites can be blocked outside of the control of users ${ }^{123}$. These are all measures assumed to be reserved for extreme cases, but any politically motivated movement is potentially at risk when using social media to organise. It is worthy to note that despite the democratic principles argued for by new media theorists, information can be censored using the very affordances that provide access. Privacy and anonymity are affordances that are not as secure as usually expected. For the JON these concerns are beyond the scope as the protest and movement were peacefully enacted in a society that allows such actions, but Biscuitwala et al. are apt to point out that Social Media sites can be circumvented by malevolent governments or powerful organisations if required. While initiatives like JON speak to the power of Social Media they have inherent risks as well and rely on trust rather than inbuilt protection

\subsection{2 'Mass' Media}

For news media there is a concern in differentiating between 'mass' media and personal $\backslash$ social media. While it was once clear that TV was mass media and a home video was personal, the same 'home movie' on YouTube can be watched by more people than a popular TV show by millions of viewers. Broadcasting is no longer an enterprise with an anticipated audience. Although it is implied by its nomenclature, scale no longer seems an adequate distinction for what is considered mass media. Understanding 'mass' media as temporal also follows in the sense that digital media does not have mass to speak of ${ }^{124}$.

\footnotetext{
122 Biscuitwala et al. 2013, 850

123 This concern has recently been reified with events in Turkey where access to social media Twitter and YouTube have reportedly been blocked through their servers (BBC.com; March 31, 2014)

${ }^{124}$ It does has mass in the physical sense, being composed of matter. When compared to the mass of conventional media which is exponentially larger, the weight is negligible.
} 
Walter Benjamin wrote about the fundamental change that reproducibility had on the $\operatorname{arts}^{125}$. With reproducibility, came the broadening of an audience and the ability for experiences to be stored and to a certain extent re-experienced. Benjamin in particular was concerned with the differences between possible experiences, and created a distinction between those that could be reproduced in those that were unique (or more unique). He suggested there was a 'cult' value to viewing the original of the work that existed only in one place ${ }^{126}$. The transformation of digital technology is one of distribution and transformation. The same easily reproducible objects become even more easily reproducible and the reproduction doubles as a form of distribution. As easily as they are reproduced, they can also be manipulated. Transformation is not only available with digital technologies; photographs can be altered manually; books can be highlighted; television programs can be spliced with differing lengths of advertisements; anything that can be created and reproduced can likewise be transformed. Digital transformations however occur at the source. Digital artifacts exist primarily as information, and their physical existence is microscopic. What we interact with are projections, but the information to build these projections can be manipulated directly. Whereas alterations made physically may assert themselves and the 'original' can be discerned, a digital alteration can be so complete that it leaves a trace only in the aspect of the artifact that is unseen (the code). Measures are often put in place to control who has access to information that is published on the Internet. Often these measures can be circumvented or hacked, but also these measures can provide false sense of control over information. This is the case in social media, where users to not directly manipulate the HTML encoding on their 'page' but rather fill content boxes, the format of which they have no control over. The simple example of this is the Facebook profile picture which appears next to any

\footnotetext{
${ }^{125}$ Benjamin 1935, 5

${ }^{126}$ Benjamin 1935, 2-4
} 
comment user posts. This profile picture is frequently updated by the user, but also by the site for any instance of its appearance. So, the profile picture that appears next to comment is not always representative of the picture as it existed when the comment was made. Incongruities the between images and content can lead to frustration of meaning ${ }^{127}$.

It is also the nature of the digital artifact as information that allows digital artifacts to be distributed quickly and easily to be projected elsewhere with ease. The number of copies is limited to demand rather than production capabilities ${ }^{128}$. For an individual it is meaningless to say that they have more than one copy of a digital artifact, one copy is the same as thousands. The number of copies, is more meaningful when referring to the number of locations from which the user can access the projection. Similarly, multiple copies may be meaningful if they have been transformed. Using the example of a photograph, the user may have larger or smaller files with differing levels of resolution, or they may have copies that have been edited for different reasons. The size of a file is pertinent to the JON conversation because different social media sites have different criteria for image size and may factor in the user's decision to embed an image directly into their post or provide a link to an external image hosting server such as Flickr or Imgur. The photographer may feel that the lower resolution available embedded directly into social media site degrades the artistic property of photograph.

Although dissemination of digital objects in most cases is far easier than the distribution of the same physical objects, when dealing with video and audio files, ease of distribution is still a factor. For example, most of video clips provided online are still hosted on YouTube servers. News media websites, particularly those associated with television networks (e.g. CBC.ca, CTV.ca), will provide their own streaming service. In part, this allows them to control the quality

\footnotetext{
${ }^{127}$ Donath and Viegas 2002, 359; Cheshin et al. 2011, 5-6

128 There are limitations such as bandwidth and storage, which should not be overlooked as there is an infrastructure involved in distributing on a mass scale.
} 
of their content, but it also allows a greater degree of control over the content itself. In order to earn advertising revenue for consumption of the content, news sites need to constrict viewership to their URL to be tracked. Content streaming through YouTube will earn advertising dollars for YouTube, not necessarily the content producer. The decision to create native affordances for rich media is largely based on server space and bandwidth, something available only to large organizations. However, there are many social media platforms that can provide both server space and bandwidth as an attraction for users to spend time on their site distributing, consuming, and communicating about digital artifacts.

Distribution of images, audio, and video also becomes problematic when dealing with device compatibility as not all mobile devices provide affordances for viewing audio and video as they would exist on a website designed for desktop use. As a result, the news article that relies on a photo gallery easily navigated on the desktop web will be missing a key element for a mobile user. Also the mobile user may be paying for their data use and choose text over a richer media that also involves more bandwidth. This concern involves both the distribution and consumption of content.

However, there may be an explanation that predates new media which can clarify the issue. In 1978, McLuhan wrote that "the key to "mass' is simultaneity rather than quantity"129 suggesting that the understanding of mass media depends not on the size of audience that can access information, but also whether they do so simultaneously. McLuhan here links time in a similar way to Benjamin's understanding of the 'cult' value, which in a digital context would mean that twitter is more of a mass medium than CBC.ca. It is unclear that McLuhan's definition can hold up to the complicated understandings of simultaneity allowed in the digital context.

${ }^{129}$ McLuhan 1978, 260 
Lance Strate contends that as well as altering our understandings of space, digital media fundamentally change our experience of time:

...the multi dimensionality of hypertext transforms the notion of text from an object held by the reader into an environment through which the reader navigates. Time can be represented in this format just as easily as space, so that the reader may explore a hypertextual network that links representations of different moments or eras ${ }^{130}$.

The preservation of digital media allows for a re-experience not dissimilar to original experience. A news article from years ago may be mistaken as contemporary if the byline is skimmed. Often they are even shared on Social Media sites without initial acknowledgment of the temporal displacement. Live webcasts and Tweets, but much of the power of 'mass' in McLuhan's sense was in the gathering of people in a shared moment listening or watching the same content. Digital content may reach very large groups, but it is contentious whether the same kind of 'mass' will really ever accumulate around objects that do not require it. With the fractured pathways available through media it is unlikely even simultaneous experiencers will consume the same content. Information consumption can also be conveniently scheduled around everyday life, and only events that have a high level of cult value, those that suffer when experienced after the fact (sporting events, crises, etc.) are necessary to experience 'live'. This can explain why the JON, which took place from January $16^{\text {th }}$ to March $25^{\text {th }}$ went from having very sporadic twitter traffic for over two months, to trending on the day of the protest ${ }^{131}$. If we consider Twitter as a mass media based on its need for simultaneity this makes sense. It also explains why the same

\footnotetext{
${ }^{130}$ Strate 2003, 372

${ }^{131}$ See Appendix C.3: Figures 29-37; This trend is seen in the volumetric timeline for the related hashtag \#idlenomore tracked by Gregoriev 2013, and Blevis 2013. According to Blevis' blog post on March 26 $6^{\text {th }} 2013$, the day of the JON protest (March $25^{\text {th }} 2013$ ) was the first day since January $28^{\text {th }} 2013$ that \#idlenomore had exceeded 8,000 tweets in a day (reaching 9,007). \#Idlenomore had fluctuating between 1,500 -2,500 tweets each day in the preceding weeks and returned to between 2,961-1,322 for the remainder of the week (March $26^{\text {th }}$ to March $30^{\text {th }}$ 2013). (Blevis, March 31, 2013)
} 
spike in the less synchronous medium of Facebook was less pronounced ${ }^{132}$. That said, there is evidence of both sites being used in both ways Facebook can be used 'live'133 and Twitter can be used asynchronically ${ }^{134}$. Despite the ability to do otherwise, there is something to be seen in the way these media are interpolated into complicated streams of time creating new understandings of 'mass' media.

\subsubsection{More Blurry Lines: Producer and Consumer}

One of the important questions in understanding digital media is the blurring line between producer and consumer. In web 2.0 users are largely expected to be both ${ }^{135}$. The difference between alternative media and mainstream media used to be one of accessibility, or hand produced pamphlets were sent out with low distribution rates. The distinction is less clear when ported to the online world, where it seems to be the real world recognition of mainstream media that distinguishes it from similarly interfaced and accessible alternative media. With online publishing readily available, distribution is no longer the issue, so it is more a question of visibility. It is easier for users to access alternative media (provided that they look for it) so it is important to understand why users would seek out alternative content. Rabble.ca's mission statement can provide some insight. The website states: "rabble.ca will draw on the real energy and power of the Internet - passionate, engaged human beings. Blurring the line between readers and contributors, it will provide a needed space for issues, a place to explore political passions

\footnotetext{
${ }^{132}$ See Appendix C.3: Figure 32; Another explanation lies in the fact that the Facebook chart only measures independent posts, not other affordances like 'comments', 'likes', and 'shares' which did increase on the $25^{\text {th }}$, but were not able to be measured using Facebook's API. On Twitter 'comments' and 'retweets' (similar to Facebook's 'share' and 'like' functions as they require very few clicks to execute and transmit in a similar way) generate as new posts and are quantifiable.

${ }^{133}$ See Appendix C.1: Figure 12; here a live TV event is discussed by many users leading up to, during, and after the event. Facebook also affords private live chat that, while outside the scope of the project, can technically create a group experience of a live event without colocation.

${ }^{134} \mathrm{~A}$ user can search previous tweets and is fed tweets of users they follow to be viewed at their convenience

${ }^{135}$ Kaplan and Haenlein 2010, 61
} 
and an opportunity to expand ideas" ${ }^{136}$. These ideals suggest reasons that users would seek out alternative media to provide a different take on a topic. Almost as a direct counterpoint to rabble.ca's mission statement, in the CBC.ca's section on credibility they write: "In a world where the line of what constitutes 'journalism' appears to be increasingly blurred, $\mathrm{CBC} /$ RadioCanada holds itself to the highest standards of journalistic practices"137. From this juxtaposition we can see that both are attempting to advocate for their strengths, and in doing so provide definitions of media based on their situations as mainstream and alternative. Rabble.ca sees their strength in participatory media, and garners its credibility from the democratization of production. CBC.ca creates for itself a defense against these blurred lines citing openness and journalistic standards and practices that create the credibility based on the experience of the source of content (being an accredited journalist or team of journalists) rather than the breadth of the source of the content (which for rabble.ca would be the large number of contributors and/or commenters). CBC.ca sets itself up as an authority to provide "the news, content and commentary and culture that Canadians need today, tomorrow and in the future"138. Rabble.ca highlights their reflexivity to user generated content and feedback, not as an added feature, but a source of content.

Palme et al 2010 looked specifically at the use of news aggregators as a potential solution to the problem of information overload. Aggregators can also be seen as a way of taking back control over media consumption. They cite the importance of 'curation and aggregation' functions they locate as previously in the domain of professional editors ${ }^{139}$. Increasingly these tasks have been performed by Internet users who want access to a greater pool of content based

\footnotetext{
136 Rabble.ca (n.d) "About"

${ }^{137}$ CBC.ca (September 16, 2013) "Terms of Use"

${ }^{138}$ CBC.ca (September 16, 2013) "Terms of Use"

139 Palme et al. 2010, 1
} 
on their interests. Importantly aggregators allow creation to be "unbundled from content production" ${ }^{\prime 40}$. They discuss the impact of providing text snippets or photos which provides advanced content that helps users decide based on their interest. While a positive effect this can also lead to free riding which occurs when users are content with snippets and do not continue on to access full content ${ }^{141}$. In their study they found that the longer a snippet the lower the likelihood that the user would click through to the source. They also found a positive relationship between snippet length and the amount of time spent using the aggregator before deciding to click through to an article ${ }^{142}$. They also suggested that very short snippets do not provide enough information to inform users as to whether they would find articles interesting causing them to spend short periods of time viewing linked articles ${ }^{143}$. Overall there was an inverted $\mathrm{U}$ relationship to time spent on the article and snippet length. There was also the same relationship between the number of articles in a topic group and the chances that a user would find at least one of them appealing ${ }^{144}$. They suggest that this is because multiple articles on the same topic seemed to users to be more important. This is important when dealing with social media in the conversation around JON because often news media will be presented with images and snippets. This research provides insight into how much information can be gleaned from snippets in order to use communication affordances within the social media site, as well as how likely these media are to encourage linking to the source.

Kaye and Johnson 2003 wrote at a time when newsmedia was largely print based and in the process of transitioning to digital content. Their concern was that digital content and internet

\footnotetext{
140 ibid.

${ }^{141}$ Palme et al. 2010, 3

${ }^{142}$ Palme et al, 7

143 ibid

${ }^{144}$ Palme et al, 7
} 
use would be substituted for time spent consuming conventional media ${ }^{145}$. They showed that internet users still relied on traditional sources, even consuming more than nonusers, which they attributed to the internet user group being more highly educated ${ }^{146}$. Different from Web 2.0 media, they describe online news as "an asynchronous, one-way source of information"147. With little interaction, there were clear boundaries as to producer and consumer. However, there were avenues for interaction using CMC. The authors also discuss chat room use, which even then was linked to media consumption behaviours ${ }^{148}$. This study, while not straightforwardly relevant to modern digital media provides both a picture of digital media contemporary to some CMC theory, and a contrast of how much the landscape has changed. Flipping the coin, theorists today are now looking to increase traffic to digital media sites to supplement or replace traditional channels.

Graham and Greenhill 2012 outline some of the current research that discusses issues surrounding the transition of conventional media to online formats. They discuss the current state of online news as convergence, where digital media are linked to other information technologies ${ }^{149}$. The authors also provide reasons users would turn to aggregators and social media as entry points for news. They suggest that aggregators present a more convenient user interface and greater content creating a "rival to newspapers" ${ }^{150}$ One of the problems they found, was that younger users were accustomed to finding content on the Internet for free and were not seen as willing to pay for news ${ }^{151}$. They note that pay walls have a very strong negative

\footnotetext{
145 Kaye and Johnson 2003, 261

146 Kaye and Johnson 2003, 262

${ }^{147}$ Kaye and Johnson 2003, 262-263

148 Kaye and Johnson 2003, 269-270

149 Graham and Greenhill 2012, 90

${ }^{150}$ Graham and Greenhill 2012, 90

151 Ibid.
} 
correlation with user adoption ${ }^{152}$, but also that more users are attracted by higher priced newspapers. They attribute this to brand recognition ${ }^{153}$. The authors suggest that important aspects of the Internet as a medium communication are that it is highly interactive and possesses simultaneity as well as increased autonomy and control when compared to conventional media. Largely the work focused on increasing traffic and readership, rather than bettering usability, but there are instances where the two goals are compatible. Graham and Greenhill lament the fact that media websites do not take advantage of the new affordances provided by digital media rather than relying on their conventional strong points ${ }^{154}$. They highlight the need to tailor products to the digital medium in order to differentiate from their analog counterparts ${ }^{155}$. This relates to the JON conversation because despite the relative lack of coverage, news artifacts were generative of a large volume within the conversation. Convergence between non digital media to digital forums provides a platform for debate amongst disparate user groups. Also, understanding why media sites have more or less traffic influences how influential their media artifacts may be when shared.

\subsection{The Effect of Affordance}

Many theorists have undertaken the project of understanding what exactly is special or unique about the digital medium. Important to this project, some of this work is involved in understanding not only what the capacities of these new technologies are, but also examining the interplay between technology use and user. Some of this work has been centered on

\footnotetext{
152 Graham and Greenhill 2012, 100

153 Graham and Greenhill 2012, 98

154 ibid. 91-92

155 Graham and Greenhill 2012, 98
} 
understanding the lag in the adoption of computing technologies for unconventional means.

Early on it seemed that there was a stigma attached to studying, or participating in online culture.

\subsubsection{Putting the 'New' in New Media}

Paglia summarizes her theories on why academics are slow to submit content to webzines citing "the blinking marginalia of advertising logos" that are reminiscent of "the banks of flashing bulbs at a casino or Penny arcade" ${ }^{\prime 56}$. This provides a tangible example of the design and format of web-based content filtering the kind of users that contribute, likely unintentionally. Paglia also notes the need for her text to be visually designed creating frequently space paragraphs in order to alleviate reader fatigue ${ }^{157}$. These adaptations not only alter the process of content editing, but also alters the conception and articulation of ideas. Kathryn Hayles argues not only that we have adapted to the use of technology, but that technology has fundamentally altered our mental circuitry. In reference to the F pattern (a pattern of eye movements for quickly scanning webpages), she adds that "reading such pages further intensifies this mode of reading"158. The more we grow accustomed to reading in this pattern, the more beneficial it is to design pages to accommodate the alteration.

Hayles further discusses the changes that digital technologies have made to the ways that human beings have learned to read and write. She suggests that there are now three ways of reading, close reading, hyper reading, and machine reading ${ }^{159}$. Close reading she defines as the traditional kind of reading that is done in the study of literature and the humanities. This is also the kind of reading that is done in most academic settings, when one needs to pay close, detailed attention to the subject matter. Machine reading is the kind of reading that is done when several

\footnotetext{
156 Paglia 2003, 270

157 Paglia 2003, 271

158 Hayles 2012, 66

${ }^{159}$ Hayles 2012, 55-79
} 
texts are sent through Computer Systems that are designed to read large volumes of text, or perform textual analysis at a speed that is only possible by using a computer ${ }^{160}$. Hyper reading is a kind of middle ground which Hayles suggests is used by people trying to assimilate large amount of information in a short period of time this is similar to what some academics do when scanning journal entries for content ${ }^{161}$. It is also the kind of work that is undertaken by casual readers of news websites scanning quickly for the important details, but not taking much time to reflect upon what they have read. This provides a challenge to conventional notions that equate analog forms of written communication with digital forms of communication through text.

Bolter problematizes some of the early attempts of exploring electronic text as merely adopting techniques from previous forms. This is certainly in keeping with early uses of computing technology, but Bolter foresaw the impact that hypertext would have on the new medium ${ }^{162}$. He argued that the shift we were experiencing with the onset of electronic writing and electronic text would be "the fourth great technique of writing" following the scroll, codex, and printing press ${ }^{163}$. In 1991, computers were still seen as tools that had as little an effect on their output as the typewriter. Bolter, however, argued that the computer “ ...offers a new surface for recording and presenting text together with new techniques for organizing our writing. In other words, it offers us a new writing space" ${ }^{\text {164 }}$. It is only by seeing computer mediation as a new writing space that our understanding of computer-mediated communication can evolve beyond generic understandings of communication. At the same time, the design of interfaces for $\mathrm{CMC}$ tend to be utilitarian aiming primarily to be the most effective at providing the affordances their users desire in order to communicate.

\footnotetext{
${ }^{160}$ Hayles 2012, 75

${ }^{161}$ Hayles 2012, 63-75

162 Bolter 1991, 6

163 Ibid.

164 Bolter 1991, 10
} 
Choudhury et al 2009 undertook a study on interestingness in conversations to see if they could glean what factors caused users to engage more in conversation on social networks. They suggest that Social media sites allow for the most communication around media uploaded by users, but that the conversations often stray from the media to other themes ${ }^{165}$ They suggested users will engage if the theme is of interest, they know the people taking part in the conversation (either personally or by reputation), or they see two or more users in an engaging dialogue ${ }^{166}$. They suggest that interestingness for a user has less to do with the user than with the communication properties of other users ${ }^{167}$. Choudhury et al 2010 echo the importance of shared media objects as a centre for rich dialogue communication ${ }^{168}$. The authors discuss the importance of affordances and modalities of online communication to understanding communication networks ${ }^{169}$. They also paid particular interest to new interactional modalities as way for users to contribute stating "the users' involvement and their contribution through non-message based interactions, e.g. digging or social bookmarking have become a major force behind the success

of these social spaces"170. There are many examples of these modalities being utilized throughout the JON conversation.

\subsubsection{Designing Affordances}

One way to understand the effect of affordances it to look in detail at specific interfaces as they are developed, tested and used. In their article entitled the "Chat Circle Series", Donath and Viegas 2002 cover a series of chat programs that they created. Their original goal was to provide spatial relations to users of group chat rooms. At the same time, they wanted to avoid the

\footnotetext{
${ }^{165}$ Choudhury et al 2009, 331

${ }^{166}$ Choudhury et al 2009, 332

${ }^{167}$ Choudhury et al 2010, 339

${ }^{168}$ Choudhury et al 2010, 8

${ }^{169}$ Choudhury et al 2010, 4-5

${ }^{170}$ Choudhury et al 2010, 8
} 
emotional confusion of having an avatar or photo of the user that could potentially provide mixed signals ${ }^{171}$. They also provide a rare insight into design decisions surrounding affordances for $\mathrm{CMC}$ and their effects. In their first iteration they used speech bubbles protruding from the user's name spelled vertically to create an abstract avatar of each user. These forms provided a 'physical' location on the screen to give users a sense of where they were in relation to other users. The interface also allowed for users to be recognized even if they did not speak, providing the opportunity for listeners rather than lurkers ${ }^{172}$. The impulse to have presence without utterance can be seen in newer CMC interfaces in the form of up voting and down voting, liking or recommending, and other unobtrusive ways of making one's presence known, but lurking is still common ${ }^{173}$. The program also involved the notion of being in or out of hearing range, which the authors note created a form of communication in the ability for users to move towards or away from people based on their interest in hearing or being heard. The first iteration of Chat Circles was centered on re-creating many of the aspects of face-to-face communication that were lost in the transition to $\mathrm{CMC}$, but the creators were aware that to be the most effective was not necessarily to exactly re-create the conditions of face-to-face communication ${ }^{174}$. The problem is, that the 'as near as possible' solutions provided by other affordances proved to confound rather than aid users' communication efforts. Chat Circles 1 was also novel in that it allowed for a history of the conversation to be viewed by users. With this affordance, users were provided with something that is not available in analog synchronous interaction. It was new to digital synchronous technologies at the time, but has since become a standard and expected affordance. The history function was fine-tuned throughout the series through user testing so the authors

\footnotetext{
${ }^{171}$ Donath and Viegas 2002, 359

172 Donath and Viegas 2002, 359

173 Soroka and Rafaeli 2006, 164

174 Donath and Viegas 2002, 360
} 
could see firsthand how the history affordance affected communication, and how the interface provided for doing so did the same ${ }^{175}$. Initially, the history refreshed and deleted at the end of a session, or as space ran out, but later iterations allow users to browse through the conversation history. The authors noticed that this changed some of the interactions, requiring users to repeat themselves less often, but also saw them reword their initial utterances ${ }^{176}$. With Chat Circles 2 , the authors further experimented with the concept of embodiment the added background images, a map of the entire chatspace, and a representation of users' movement through action tracing ${ }^{177}$. With Chat Circles 2, not only were the users able to experience communicating in space, but their spatial movements were used as another form of meaning creation ${ }^{178}$. The authors discuss how users could move away from someone who would was irritating them (and be seen doing so). The images in the background also served as loci for conversations on relevant topics ${ }^{179}$. This aspect is something that has been observed as central to modern Social Media sites ${ }^{180}$. The third relevant program the authors created was Chatscape wherein users could alter their abstracted appearance to communicate certain important details about themselves, and the details could be altered by others. The shape and the colour of the users could be altered by who they talk to and the impressions they left on others ${ }^{181}$. This article is unique and important because it was a longitudinal overview of a series of communications programs. It shows how our adaptive techniques adjust to specific interfaces and change with related interfaces over time, highlighting a dimension that is overlooked in much of the scholarship surrounding CMC and Digital media.

\footnotetext{
${ }^{175}$ Donath and Viegas 2002, 361-362

176 Donath and Viegas 2002, 361-362; 364

${ }^{177}$ Donath and Viegas 2002, 363

178 Donath and Viegas 2002, 365-366

${ }^{179}$ Donath and Viegas 2002, 362-363

${ }^{180}$ Choudhury et al. 2009, 331-332

${ }^{181}$ Donath and Viegas 2002, 366
} 
Where the creators of Chat Circles focused on space in the online world, there is an equally complicated understanding of time. Ogura et al. 2010 designed an interface that attempted to deal with the different kind of temporality that exists in the digital world called Kairos chat. The designers of Kairos chat were looking to provide an affordance for users to be able to filter out the main discussion from sidebar comments ${ }^{182}$. They based their system around the idea of experiential rather than absolute time. This system involves two 'lanes' of text in which the text remained for different periods of time. Any text that was directly related to the main topic of conversation would go in the slow lane, and any tangents or sidebar conversations would go into the fast lane. This system was designed around the Ancient Greek concept of Kairos time which, contra Kronos time, is experiential rather than absolute ${ }^{183}$. The goal was to emulate an analog conversation, where the psychological or Kairos time would naturally occur. Things that are off topic are more ephemeral, take less time to process, and are not required for future reflection. The designers performed a study involving three sessions, two using a baseline chat and one in between those using Kairos chat. Users were instructed as to how to submit and scroll through messages, but not which lanes should be used for which types of message ${ }^{184}$. They found that users "naturally accepted the multiple streams of time without any concrete instructions on how to use each lane" 185 . This left the creators in a position to further improve the system for future testing confident in the concepts viability. Kairos chat was not used in the JON but it serves as an example of interface design based on a psychological need. Users currently rely on affordances that are not tailored to the task of filtering content based on relevance and

\footnotetext{
${ }^{182}$ Ogura et al. 2010, 3724

${ }^{183}$ Ogura et al. 2010, 3722

${ }^{184}$ Ogura et al. 2010, 3725

${ }^{185}$ Ogura et al. 2010, 3725-3726
} 
frequently express distress at the information overload ${ }^{186}$. This suggests that this overload is not inherent to digital media per se, but may be mitigated by the delivery system. Kairos Chat provides a concrete example of the strategy of segmentation for which users currently are required to create (or allocate) their own space.

Affordances can refer to programmatic capacity, but also to physical systems. Oviatt et al. 2012 looked into whether the physical affordance of an interaction affected the kinds of meaning that were produced. They maintained that users have "perceptually based expectations about objects" and believe there are specific ways to successfully interact with different affordances ${ }^{187}$. They suggest these beliefs lead to behavioural attunements that mean users will associate affordances with particular tasks. In particular their goal was to show that users of a pen interface would use more non-linguistic markings and the keyboard interface would constrain users to more linguistic utterances ${ }^{188}$. Their work suggests that input capabilities influence the types communication used and raises questions about how this affects new devices that increasingly move away from physical keyboard inputs. In the JON conversation it is probable that many posts were submitted on mobile platforms and the perceived capabilities of the device inputs may have played a role in the communications affordances users chose to utilize.

\subsubsection{User Determination}

While affordances can either be provided or not by technology, often important affordances are unintentionally provided by designers and are either found or invented by users. This is especially the case for communications technology, where designers have less

\footnotetext{
${ }^{186}$ Hayles 2012, 55-56; Bauldrillard, 63

${ }^{187}$ Oviatt et al 2012, 22:3-22-4

${ }^{188}$ Oviatt et al 2012, 22-4
} 
understanding of exactly what it is about the tools they create that allow us to make and understand meaning. For Hutchby 2001, designers have control over the intended use of a system but have little control over communicative affordances. "The affordances of an artifact are 'found' by it users in the course of their attempts to use it for various ends" ${ }^{189}$. The telephone, for instance, was invented for utilitarian purposes to run households and communicate for business. The idea that users would 'chat' to their friends at a distance is an example of a 'found' affordance $^{190}$. This is the case with many kinds of technology, but is especially prevalent with digital tools that are available online. With little capital investment or infrastructure on the part of the user, affordances are much more easily found. For example, Jose Van Dijck 2013 suggested a similar mechanism was at work in the adoption of Twitter. After its emergence, Twitter was "...a service in search of the user application" ${ }^{191}$. He suggested that Twitter was created for its compatibility with mobile phones, but its adoption on other platforms and for other purposes, such as microblogging was user determined ${ }^{192}$. Viewing these Web 2.0 technologies in this way allows us to see not only how users are making use of the technology now, but also to infer certain things about past use patterns. For example, the way emoticons are used today, in that they are substituted out for animated pixelated images provides proof that at one point before the images existed there existed an impulse by users to create shapes using punctuation. This is both an example of users finding an affordance within the system (images from punctuation) and users finding a creative escape for a constraint of the system. An important aspect that drives this user determination is that very often with new technologies, especially

\footnotetext{
${ }^{189}$ Hutchby 2001, 123

${ }^{190}$ Hutchby 2001, 123

191 Van Dijck 2013, 225

192 Van Dijck 2013, 224-225
} 
communications technologies, what is promised by a system is not what it affords ${ }^{193}$. Hutchby uses the example of videophones that do not allow for the "reciprocity of perspective" that occurs in face to face communication as the camera is not directly aligned with the gaze. This alteration of the analog creates new opportunities for creating and altering meaning with the new technology. The backdrop of your webcam frame can be edited carefully allowing much more control over the presentation of your surroundings than can be expected in real life. Creating meaning out of these differences takes time, and now these 'found' affordances are not attributed to any individual user. Instead they are developed over time and negotiated amongst those who have adopted the new technology. Found affordances, in the context of communication, can manifest as conventions that are used to overcome a particular obstacle. This could be seen in the early instant messaging program Usenet when it was used exclusively in Universities. The problem with the system, articulated by Bethany Nowviskie 2012, was that every September new students who had not been initiated into the system would disrupt the natural mechanisms that the more experienced users had evolved. After the first month or so, new users would either give up or have adapted to the conventions. There was an etiquette to the compensatory methods, and that etiquette was socially mediated ${ }^{194}$. Even after the 'Eternal September' when Usenet was released to allow a never ending stream of initiates, these processes whereby one is socialized into the acceptable adaptations of the medium persist and continue to evolve the way users learn to communicate online. Donath and Viegas observed their users policing conduct with found affordances. They saw users of their Chat Circles 2 program using their action traces that were

\footnotetext{
193 Hutchby 2001, 126

194 Nowviskie 2012, 244
} 
designed to show their movement to actively block the passage of other users; an affordance they had not anticipated ${ }^{195}$.

The design of affordances is not the only process by which they are created. Often they are found or created, and then negotiated by users. For instance, in any text based chat the system is usually designed as being synchronous or asynchronous, however, as is alluded to by Kairos Chat, this does not fulfill our communications needs. As a result, it is very common to use synchronous communication, such as the chat system, in an asynchronous way. This is done by having multiple windows open and holding several of these conversations with different subjects parsing attention back and forth between the conversations. This kind of use was established and mediated by users rather than the systems or the system designers.

\subsection{Computer-Mediated Communication}

Emerging from the early studies on Computer Mediated Communication (CMC) researchers began to look at the question of how a person could express the non-verbal cues so essential for communicating emotion through the flattened, media-poor text-only CMC. Computer mediation theorists interested in the psychology of communication made use of negative and positive affect conditions to examine how emotions were communicated through textual interaction in CMC. Their observations provide us with an understanding as to the mechanism of this communication as well as some pertinent observations about how users adapt to the tools at hand when communicating in constrained channels. Conventional psychology suggests that communication of emotions relies heavily on nonverbal cues ${ }^{196}$ which CMC media traditionally lack. It would seem, then, that the communication of emotions would be difficult if not impossible in less media rich environments like text only chat. However, recent research has

\footnotetext{
195 Donath and Viegas 2002, 366

${ }^{196}$ Walther et al 2005, 36 ; Hancock et al 2008, 295
} 
turned that assumption into question, proving not only that people are able to infer emotional content from CMC overtly, they do so so naturally that the phenomenon of emotional contagion can occur ${ }^{197}$. Inquiries into the mechanisms and psychology for emotional communication in CMC are unfortunately focused on the participants' emotional states and less so on the particular affordances and mediation of their interactions. However, the tradition of scholarship provides a good contextual understanding for the foundational issues surrounding CMC. The following is a review of that scholarship beginning with Walther's work on Social Information Processing theory (SIP) which provides the mechanism for later understandings of emotional communication through the constrained medium.

Very early understandings of computer mediated communication centered not on person to person communication, but on communicating with computers themselves. More specifically with their programming. Gaines and Shaw 1984 wrote an early work about the exciting prospects of communicating with computers. To provide perspective, the authors were working under the assumption that eventually natural language systems would be the primary interface for computing technology. Their work was an attempt to train users to understand the subtleties of conversing with these new machines. Gaines and Shaw imagine computers as enabling users to “...interact with a program in a similar way to that which we interact with the person"198. The authors even go so far as to suggest that this interaction is the same as conversing with the programmer as the programmer has the ability to encode their expertise and 'respond' ${ }^{199}$. For Gaines and Shaw it was that the encoding of information could be re-experienced and interacted with by recipients that made communication through computers unique ${ }^{200}$. This is important to

\footnotetext{
${ }^{197}$ Studies includes: Hancock et al 2008; Cheshin et al 2010; Pirazdeh and Pfaff 2012

${ }^{198}$ Gaines and Shaw 1984, 20

199 Ibid.

${ }^{200}$ Gaines and Shaw 1984, 21
} 
understanding modern computer mediated communication because it highlights an important aspect that is often overlooked. If we see computer mediated communication as constrained person-to-person communication we neglect what the mediation adds. For example, webpages are carefully designed with various factors in mind depending on the context. While aesthetics is part of the goal, often so is branding. Colour schemes are a very good example of how designers communicate by creating an experience for users that is largely unnoticed. Even before the adoption of large-scale two-way communication using computers, Gaines and Shaw saw computer communication as already richer than unidirectional media such as television and books $^{201}$. The interplay they saw was between the user and the machine, whereas in later understandings of interactions between users the machine is seen as a barrier or an enabler; not an enhancer. The goal of later CMC technologies would be to eliminate any interference from the mechanisms of the technology. The vision of the seamless connection between people using computing technology to provide a new location for communication, but not otherwise alter the human to human interaction was seen as the endgame. $\mathrm{CMC}$ research began looking at the medium as something that fell short of this ideal, and to a large extent moved away from trying to understand what the computer added.

\subsubsection{CMC: Constraints and Adaptations}

In 1992, Walther introduced his Social Information Processing theory (SIP) and began to undertake a detailed exploration of the mechanisms by which users communicated through CMC. He suggested that users would adapt to the medium and find ways of expressing themselves through the available affordances and their experiences with other users ${ }^{202}$. Through this mechanism we see how influential the use of technology, rather than the affordances of

\footnotetext{
${ }^{201}$ Gaines and Shaw 1984, 20
}

${ }^{202}$ Walther et al. 2005, 37 
technology is on the effect of the technology. Much of the communication in face-to-face (FTF) interactions is attributed to non-verbal cues such as facial expression and body language. However, in computer-mediated communication (CMC), these non-verbal cues are not obviously present. Walther et al 2005 describe that in understanding the mechanism of CMC there are two possibilities. Some claim that "the absence of nonverbal vocal and physical cues denies users important information about partners' characteristics, emotions, and attitudes, resulting in less sociable, relational, understandable, and/or effective communication" 203 . The second viewpoint is that people will adapt, in absence of these cues, and find ways of communicating through whichever format they have available ${ }^{204}$. Walther argues for the latter view, and endorses SIP theory which he put forward in his previous works. Despite the phrasing of the first claim, it is clear that $\mathrm{CMC}$ is effective, understandable, and sociable enough to be used regularly by multitudes of people. As such it is almost a given that users will adapt to the absence of conventional cues. However, given that Walther is writing from a psychological point of view, it is important to prove that this aspect of communication does exist. Also, despite the anecdotal evidence, there are many who still believe that the deficiencies of CMC cannot be overcome to the point that it is truly effective.

Hancock et al. 2007 pick up on Walther's argument for SIP and particularly the suggestion that "users employ the verbal cues present in CMC to convey relational information that may normally be transmitted via nonverbal cues in face-to-face contexts" ${ }^{205}$. They endeavour to identify some of the specific mechanisms through which these cues are transmitted. Their study showed four strategies that were used towards this end. These were 1) changing the degree to which the expresser agreed with their partner; 2) using more or less negative affect

\footnotetext{
${ }^{203}$ Walther et al. 2005, 37

${ }^{204}$ Walther et al. 2005,38

${ }^{205}$ Hancock et al. 2007, 929
} 
terms, but not positive affect terms; 3 ) increasing the use of punctuation by positive emotion expressers; and 4) engaging through speed of response and verbosity ${ }^{206}$. It can be seen by the fourth strategy that users were not only using the content of their utterances, but the format through which they were transmitted. The study also showed that despite the fact that these strategies exist also in face-to-face communication, they needed to be accentuated to be effective in $\mathrm{CMC}^{207}$.

In their 2008 study Hancock et al. look into two further questions stemming from their conclusions about Social Information Processing theory (SIP) from previous work. They aim to answer "1) how are emotions expressed in CMC when participants are actually feeling an emotion? and 2) can their partner sense this emotion?"208. They were unable to locate the mechanism for emotional contagion, but their results showed that emotional contagion could be detected using only text based CMC. They make two claims about what their study shows. First they state that "an important advance was that the emotion detected in this procedure was an experienced emotion rather than an acted emotion, suggesting that emotions can be reflected in CMC even when no goal is in place to express the emotion" 209 . This is important if we are to look at $\mathrm{CMC}$ as a natural communication medium. Adaptive strategies for conveying emotion, as in face to face communication must be intentional and unintentional. If CMC only allows us to express emotions we intend to, as is often assumed, the mediation of computer interfaces will always interfere with natural communication, no matter how advanced our adaptive strategies. Hancock et al 2008 claim that this is not the case; the claim they advance is that there are unknown mechanisms and therefore nuanced ways that we can communicate emotions through

\footnotetext{
${ }^{206}$ Hancock et al. 2007, 931

${ }^{207}$ Hancock et al. 2007, 931-932

${ }^{208}$ Hancock et al. 2008, 295

${ }^{209}$ Hancock et al. 2008, 298
} 
CMC, even when only text-based. Hancock et al 2008 claim "the[ir] second, and perhaps most important contribution, was that emotional contagion occurred between partners" ${ }^{210}$. This revelation was very important to the psychological understanding of CMC, but also for this endeavour, as it provides evidence of users adapting to the medium beyond the designed affordances and expectations thereof.

Byron 2008 echoes the cue-deficient argument for CMC. She discusses proponents of rational choice models giving the example of information richness theory ${ }^{211}$ which brings up an interesting argument around channel or media choice. They suggest text-based media were not particularly rich ${ }^{212}$. It is suggested that given the constraints of certain communication media (either between FTF and CMC or within CMC itself) users will opt for channels based on different communication needs. Given the diversity of text-based media (level of synchronicity) and even multimodal media in CMC, if proponents of SIP are correct in suggesting that there is a kind of adaptation to the media this would mean that different media would develop differently as they are used more or less often for certain communication tasks. It also brings in the necessity to look at channel choice as a factor in interpreting meaning or conveying emotion. For example, users may make such choices based on synchronicity as well as affordances. If a user wants a quick active response they will choose a channel that affords high levels of synchronicity and alerts or notifications. For an idea that requires careful thought, the less synchronous channel, where longer and slower replies are expected, would be used. When deciding based on affordances, the user might choose a forum that allows the sharing of images more easily if they have images they feel like sharing, or feel their ideas are better expressed visually. While Byron

\footnotetext{
${ }^{210}$ Hancock et al. 2008, 298; Emotional contagion is when subject contracts an emotional state from exposure to a compatriot who is feeling, but not explicitly expressing that emotion. (Ibid.)

${ }^{211}$ Byron 2008, 311; Information richness describes the quality of a communications media based on its available affordances for communicating information (Ibid)

212 Byron 2008, 317
} 
is dealing specifically with $\mathrm{CMC}$, this logic of channel functionally extends beyond conventional $\mathrm{CMC}$ to include richer digital communications channels, as well as the choice to choose a digital channel in the first place.

While Byron's work attests to emotional communication in email being more difficult than FTF to communicate emotion, she suggests that "emails may contain nonverbal cues that have parallels in face-to-face communication, such as emoticons to symbolically convey emotion, asterisks to provide emphasis, or all capital letters to indicate emotional intensity"213. Bryon 2008 goes on to coin the idea of the 'neutrality effect' whereby receivers of emails will neutralize positive emotional cues in emails which she attributes to the act of email communication being "duller and less stimulating"214, than FTF communication. She also suggests that there is a negativity bias that intensifies negative emotional content of an email upon reception while simultaneously undervaluing the positive emotional content ${ }^{215}$. While Byron discovered this effect in emails, it has since been attested to in other forms of $\mathrm{CMC}^{216}$. It is also fair to consider that wall posts and messaging in social media bear resemblance to some form and usage of email. It could be argued that they developed from the impulse to use email in different ways. As such, studying email can provide insight into these kinds of activities as well.

Cheshin et al. 2011, following this work, undertook a study of how virtual teams process emotional language in the absence of non-verbal cues. In doing so they provide a good overview of the work that has been done to date on understanding the communication of emotions through text based CMC. They described text-based communication as 'poor' based on Daft and

\footnotetext{
${ }^{213}$ Byron 2008, 312

214 Byron 2008, 312

215 Byron 2008, 312-313

${ }^{216}$ In IM via Pirazdeh and Pfaff, 2012; and Hancock et al 2008
} 
Lengel's 1986 'media richness' analysis ${ }^{217}$. They described the neutrality bias suggested by Byron, which suggests that people rarely recognize and instead tend to neutralize positive emotions $^{218}$. Coupled with this is Byron's the negativity bias, whereby negative emotions are intensified when recognized ${ }^{219}$. They cite Hancock et al.'s 2008 study on emotional contagion, and drawing on Festinger 1954 and Van Kleef 2009 they suggested "that people use the emotions of others as input when assessing how they themselves should feel”220. Their hypothesis also heavily relies on attribution theory, which suggests that users will look for the reasons behind the actions of others even with very little evidence by which to understand them ${ }^{221}$. In CMC this is influential because of the propensity for unintended sources of meaning to be created ${ }^{222}$. The goal of their study was to demonstrate that emotional contagion is transmitted through virtual communication and to explore how the transmission of it is affected by incongruous behaviour. They put forward four hypotheses, the first that emotional contagion will occur in their study, second and third that being resolute and being flexible will be interpreted as being angry and happy respectively, and the fourth being that incongruity between the text based emotional signals and behavioural emotional signals will have a negative emotional response by team members ${ }^{223}$. They suggest that their results show that anger and happiness can spread in virtual teams using only text based communication. They also found that the greatest negative emotions surfaced when there was a mismatch of verbal and behavioral

${ }^{217}$ Cheshin et al. 2011, 3

${ }^{218}$ Cheshin et al. 2011, 3

${ }^{219}$ Byron 2008, 312

${ }^{220}$ Cheshin et al. 2011, 4

${ }^{221}$ Cheshin et al. 2011, 4

${ }^{222}$ In their study, Cheshin et al 2011 intended to prove that resoluteness and speed of response will be seen as alternative cues but in their attempt to control the experiment, did not consider the possibility that these cues may exist unintentionally in an uncontrolled environment. (Cheshin et al. 2011, 5-6)

${ }^{223}$ Cheshin et al. 2011, 4-5 
cues $^{224}$. This is interesting for its confirmation of some of the mechanisms through which emotions are transmitted and perceived. This is of particular interest when considering that alongside the digital conversation of JON were events in the real world that required concrete actions. Also, being located mainly in social media, incongruities could be spotted between behavior alluded to on a user profile and utterances.

Kramer (2012) performed a study using Facebook to test if emotional contagion could be transmitted through unidirectional Facebook status updates to a user's friends. In this case the communication is a projection rather than a two way interaction, but Kramer found that negative and positive updates would affect friends status updates for three days following the positive or negative $\operatorname{post}^{225}$. While they could not definitively ascribe this effect to the posts alone, their statistics do show a significant correlation ${ }^{226}$. They also looked in to a technique they referred to as "emotional suppression where negative words would be suppressed by positive posts from friends. They looked into whether this technique was effective and found that positive words did indeed "buffer[] their friends against negativity over the same timeframe [Days 1-3]"227. This is pertinent because Kramer's work shows a potential instance of CMC producing emotional contagion in an asynchronous and non-conversational setting akin to the majority of digital communications present surrounding the JON.

\subsubsection{CMC: Impersonal or 'Hyperpersonal'?}

Walther 1996 suggested that despite the dearth of non-verbal cues in CMC, that users were in fact able to more eloquently communicate certain aspects of their personalities to others. In many ways users were seen using the mediated format to express their true selves, uncoloured

\footnotetext{
${ }^{224}$ Cheshin et al. 2011, 13

${ }^{225}$ Kramer 2012, 767-770

${ }^{226}$ Kramer 2012, 769-770

${ }^{227}$ Kramer 2012, 768
} 
by external cues. Citing a study by Kallick, Walther notes that even "users with little experience in CMC 'were better able to plan, and had increased opportunities to self-censor. With more time for misconstruction and less stress of ongoing interaction, users may have taken the opportunity for objective self-awareness, reflection, selection and transmission of favorable cues" ${ }^{\prime 228}$ Walther terms this enhanced connection 'hyperpersonal' suggesting that it is in the nature of CMC to exceed FTF interpersonal communication ${ }^{229}$. He suggests that in both group and dyadic communications "these effects are illuminated by examining processes related to the interactions of communication media with source, receiver, channel, and feedback processes, which extend our abilities to perform interpersonal functions in heightened or augmented ways via CMC"230. Conventional understandings of Social Presence Theory ${ }^{231}$ suggest that "the fewer channels or codes available within the medium, the less attention that is paid by the user to the presence of

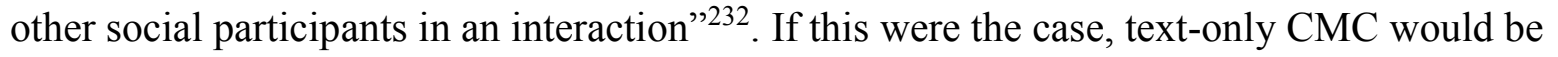
considered highly impersonal. But it is the very limitations of CMC, Walther argues, that enable us to create more considered (and accurate) self-representations ${ }^{233}$. Users are able to identify individuality and personality of others and project the same in themselves because of the constraints of text-only CMC.

Postmes et al. 1998 take a different view of identity creation using CMC and suggest that it bears a depersonalizing quality causing users to de-individuate and reinforce group salience ${ }^{234}$. For the authors, "depersonalization refers to the tendency to perceive the self and others not as individuals with a range of idiosyncratic characteristics and ways of behaving, but as

\footnotetext{
${ }^{228}$ Walther 1996, 490

${ }^{229}$ Walther 1996, 5

${ }^{230}$ Walther 1996, 5

${ }^{231}$ Attributed to Short, Williams, and Christie, 1976 in Walther 1996, 7

${ }^{232}$ Walther 1996, 7

${ }^{233}$ Ibid, 33

${ }^{234}$ Postmes et al 1998, 697
} 
representatives of social groups or wider social categories that are made salient during interaction"235. The SIDE model, put forward by Postmes et al. 1998 provides a way of understanding $\mathrm{CMC}$ as containing a myriad of social cues that are interpreted by users as indications of characteristic norms for social groups. Postmes et al. 1998 suggests that anonymity in computer mediated communication increases susceptibility to "the influence of norms, social attraction to group members, stereotypes, and ingroup favouritism"236. Essentially, what the authors show is that the very traits purported to provide liberation from conventional social boundaries may instead end up reinforcing them. Rather than getting to know the real person who in a 'real world' context would have to defer to societal norms, the deference is shown by the research to be increased ${ }^{237}$. Recognition of group mores creates greater 'us' vs. 'them' mentality as well as higher affinity based on ingroup recognition ${ }^{238}$. So, rather than 'hyperpersonal' connections CMC interactions are based on user's affinity with potentially polarizing group identities. This can be seen in the comments sections of mainstream newsmedia online. Often adversarial commenters will use stories as a platform to rehash debates based on political or social allegiances. The same polarized debates are rehashed in the comments sections of unrelated articles with users finding some way to spin the content to either side either incurring the wrath of other users who identify with opposing positions, or support from those who agree. Most conversations using this tactic end with both sides further entrenched attesting to the SIDE model. However, these claims are based on expectations of anonymity which are not always available in modern multimodal and social media linked CMC.

\footnotetext{
235 Ibid., 698

${ }^{236}$ Postmes et al. 1998, 699

${ }^{237}$ Postmes et al. 1998, 697

${ }^{238}$ Postmes et al. 1998, 709
} 
Henderson and Gilding 2004 undertook a qualitative analysis wherein they interviewed and examined the idea of 'hyperpersonal' online friendships. They were building upon previous work by Walther in 1996, which suggested that two main features of CMC are that communication is potentially asynchronous and that non-verbal cues are lessened. Their findings agree with Walther's assessment, but provide insight into the effects of the constraint. Asynchronous exchange is seen as a benefit to participants who want to type exactly the right words before sending ${ }^{239}$. The choice of response time was considered especially beneficial. Other benefits of CMC were also described. Some participants noted that on-line the ability to find people whose interests so closely matched their own was much higher. They also mention that users felt more comfortable taking risks, and disclosing aspects of themselves ${ }^{240}$. One participant noted that "'real life was more awkward than online"'241. They suggest that CMC can be an excellent platform for creating 'pure relationships'. Henderson and Gilding show some interesting reasons why despite lacking communicative affordances, text based CMC can be boon to certain kinds of people. It provides insight especially into why CMC can be a good tool for users to educate themselves and participate in something like the JON online. If they felt pressured in one setting to remain silent about their feelings about the protest, they may feel less pressured to do so in the relative anonymity of CMC. However, Henderson and Guilding note this liberation can lead also to the problem of unrealistic representation. Some of their participants mentioned that if they were making up for a deficiency when using CMC, they would not be able to transition the friendship to $\mathrm{FTF}^{242}$. Someone who felt uncomfortable speaking out in person, despite their ability to do so online, may feel even more intimidated

\footnotetext{
${ }^{239}$ Henderson and Gilding 2004, 490

${ }^{240}$ Henderson and Gilding 2004, 498-499

${ }^{241}$ Henderson and Gilding 2004, 495

${ }^{242}$ Henderson and Gilding 2004, 496
} 
doing so due to the discrepancy between their represented self online and their perception of their 'real world' self.

Chan and Cheng 2004 did a comparative study of online and offline friendships. They write that "The degree of social presence depends on the number of channels or codes available within the communication medium" 243 . As a result CMC is usually considered to have a low social presence. They cite a 1992 study by Walther where he "suggested that the critical difference between FTF and CMC is the rate [of exchange], but not the capability. Although the limited bandwidth of CMC offers less total information per exchange than does FTF exchange, relational communication of $\mathrm{CMC}$ can grow and become comparable to that of FTF, given sufficient time and message exchanges"244. This led them to postulate that while online friendships would begin deficient these deficiencies would flatten over a long enough length of time $^{245}$. Interestingly they found that a sub-set of the on-line friendships, cross-sex friendships were of a higher quality than the same type off-line ${ }^{246}$. This brings some interesting insight into the role that gender plays in the communication, as well as how this may differ in different cultural spheres where gender and social class present greater or lesser barriers to friendship. Some of the online locations for conversing about the JON provide a place for people to connect over a shared ideal, despite their gender, socioeconomic background, race, or any other discriminating factor. Other locations are implicated in social constructions that may still hinder user interactions. Some users may find themselves feeling more liberated in the online world. The Facebook group page show evidence of close interpersonal connections. Its longevity as an

\footnotetext{
${ }^{243}$ Chan and Cheng 2004, 321

${ }^{244}$ Chan and Cheng 2004, 321

${ }^{245}$ Chan and Cheng 2004, 307-308

${ }^{246}$ Chan and Cheng 2004, 317
} 
active site also shows that it is a location not only for the event, but also a site through which to nurture online friendships.

Amichai-Hamburger and Furnham 2005 survey several works that view CMC and the internet in general as a space for negative, hostile and difficulty creating meaningful relationships ${ }^{247}$. However, they suggest that rather than being devoid of emotional and social cues, "it is equally likely that electronic communication helps in the acquisition, practice and improvement of social skills" ${ }^{\prime 248}$. They suggest that internet users are empowered by three factors 1) Anonymity; 2) Control over the interaction; and 3) Finding similar others ${ }^{249}$. With most modern Social Media sites anonymity is largely abandoned by the majority of users. It is still possible to forge your identity online quite successfully, but it is not possible to completely reinvent yourself ${ }^{250}$ as the authors suggest ${ }^{251}$. Regardless, control over the interaction is still a very important aspect of $\mathrm{CMC}$ and has been cited as a reason for choosing it over other communication media ${ }^{252}$. In his 1996 study Walther found that users with little experience in CMC "were better able to plan, and had increased opportunities to self-censor. With more time for misconstruction and less stress of ongoing interaction, users may have taken the opportunity for objective self-awareness, reflection, selection and transmission of favourable cues"253 The quality of 'finding like others' will be more or less important given the ability of doing so in the 'real' world, and the ability to adequately communicate with them. In the case of the JON, the organizers were able to draw on the larger community recently solidified by the idle no more

247 Amichai-Hamburger and Furnham 2005, 1033-1034

${ }^{248}$ Amichai-Hamburger and Furnham 2005, 1035

${ }^{249}$ Amichai-Hamburger and Furnham 2005, 1035

${ }^{250}$ For example: "Susan Herring has demonstrated that gender remains largely apparent in email, list serves, chat rooms, and USENET postings, and so on, contrary to the prevailing belief that CMC provides users with a 'gender blind' environment that might liberate women from the patriarchal patterns of discrimination and oppression in the 'face-to-face' world." Ess 2002, 219

${ }^{251}$ Amichai-Hamburger and Furnham 2005, 1033

${ }^{252}$ Henderson and Guilding 2004, 490; Amichai-Hamburger and Furnham 2005, 1035

${ }^{253}$ Henderson and Guilding 2004, 490 (Quoting from Walther 1996) 
protests, and reach out to find supporters for their cause in a way that would have been logistically impossible for people in their situation without the resources of Internet communication. This phenomenon has also been cited in the context of the Egyptian uprising that not only was blogging able to connect otherwise unconnected individuals, it also connected ideas that were held strongly by separate groups that had common aims ${ }^{254}$.

Walther wrote a follow up to his earlier works in 2009 to attempt to locate his SIP theory amongst competing understandings of CMC such as SIDE. He suggests that it is not as simple as choosing one side or the other, and in modern CMC technologies there are layers of constraint and affordance that can provide the environment for many of the understandings to be correct ${ }^{255}$. The theoretical pluralism stems from the moving boundaries of CMC (dyadic vs. group; anonymous vs. social; etc.). As a result, Walther argues the need for inquiry into where these boundaries lie in relation to which theories best describe behaviour in $\mathrm{CMC}^{256}$. However, Walther describes well why even this goal may prove elusive:

...CMC itself is diversifying and normalizing. There are many forms of it, and some have become so ubiquitous that they are almost unnoticeable. Some would suggest that the next wave of CMC research should examine radical multimodality: Since people communicate within any single relationship across many media (social networking sites, e-mail, text messaging, blogs, picture-sharing, games, etc.) we need to see how all these media fit together and define social life. While the premise is interesting, the goal seems ill-defined. What are we looking for? How will we explain it in such a way that we do not have to abandon explanations when the next killer app comes along? ${ }^{257}$

Here he rightly states that these boundaries are moving targets that scholarly endeavours lag behind. However, we can make headway in continuing not only to take stock of the $\mathrm{CMC}$ environment at given times, but also how these changes and new understandings fit

\footnotetext{
${ }^{254} \operatorname{Lim} 2012,237$

${ }^{255}$ Walther 2009, 748-749

${ }^{256}$ Walther 2009, 750

${ }^{257}$ Walther 2009, 751
} 
not only with future directions, but past theories ${ }^{258}$. We should not discard old theories because boundaries change, but recognizing when it is the boundary, not the activity that has changed. Much like the way communications affordances are designed, Walther suggests scholars may choose "E: All of the above"259.

\subsubsection{Lurking}

One of the key aspects of CMC and digital media is the interactivity dimension.

However, while Social Media sites and websites provide for users to interact, there are still many who are considered 'Lurkers'. For Soroka and Rafaeli, "lurking is a participation in a computer based forum without posting to it" ${ }^{\text {260 }}$. While lurking behaviour is the expected norm in traditional newsmedia, digital media, like SMSs and online forums thrive on interaction. Despite this, most of forum users continue to 'lurk' rather than posting ${ }^{261}$. In the context of inspiring users to get active and be vocal for a particular cause, this can be seen as a negative behaviour, but there are some important lessons available in users' reasons for lurking behaviour. Many 'lurkers' use silent observation to learn about a new topic or community ${ }^{262}$. Even without contributing this can lead to lurkers finding a sense of community and belonging ${ }^{263}$. Another reason given for lurking is information overload. The number of users in a forum has been shown to decrease the interactivity; busier communities tend to acquire a greater number of lurkers $^{264}$. One of the goals of this project is to show that digital communications occur using more than just utterances. One of the powerful aspects of Social Media is the size of audience that is possible. Not every user will interact, but even lurkers consume information, and even feel

\footnotetext{
258 Ibid, 751

259 Ibid, 748

${ }^{260}$ Soroka and Rafaeli 2006, 166

${ }^{261}$ Soroka and Rafaeli 2006, 163

${ }^{262}$ Soroka and Rafaeli 2006, 164

263 Soroka and Rafaeli 2006, 164

${ }^{264}$ Soroka and Rafaeli 2006, 164-165
} 
affinity with online groups ${ }^{265}$. Also, several of the affordances provided by the digital media used in the JON conversation provide for low richness interactions such as liking, recommending, and up-voting which provide users with the ability to lurk, while still creating an impression of their presence or support. The JON Facebook group page has upwards of 33,000 members, many of whom will never have posted anything to the group ${ }^{266}$. They may have read about the group and gained a sense of community while doing so. Also, each time one of those members joined it would have been posted as a notification to appear in the news feeds of the friends of most of the new members. It also continues to reside on their home page along with their other information. Even 'lurking' opens the door for a large number of other 'lurkers' to walk through.

\subsubsection{Relating and Creating Our 'Self' Online}

Hunt et al 2012 cite several studies that suggest that SNSs" are primarily used "for purposes of socialization and identity construction" ${ }^{\text {"67 }}$. The goal of the work was to outline the motivations for users to remain on social media for extended periods and the relationship to communication affordances. The authors note that "SNSs now account for nearly a quarter of the time spent by users online"268. They suggest that users often initiate a session for interpersonal communication and continue on as they see it as a source of entertainment ${ }^{269}$. Importantly, this shows the impact of communications affordances in channeling information seeking activities. It also shows that user's relationships and use of SNSs change over time, however, the authors admit that this is likely related to the fact that the affordances and user base of the site are likewise in flux. The difficulty of capturing exactly what is and was available to users confounds

\footnotetext{
${ }^{265}$ Soroka and Rafaeli 2006, 165

${ }^{266}$ The number of group members exceeds the number of posts (this is exclusive of liking and commenting behaviour which could not be measured)

${ }^{267}$ Hunt et al 2012, 187

${ }^{268}$ Hunt et al 2012, 187; the authors cite a Neilson 2011 study

${ }^{269}$ Hunt et al. 2012198
} 
many a study (this one included). They also refer to self-expression as a significant predictor of Facebook use. The authors attribute this to an effect shown through previous research that suggests young adults use SNSs as loci for managing their identities ${ }^{270}$. This would suggest that identities created online bear relation to offline identities and vice-versa.

Mona Sakr 2012 discusses the formation of the self in the digital world. This is an important direction for this conversation because the mechanism for conveying meaning are intrinsically linked to the personalities of the individuals that do so. She also looks at "the possibility that new technologies will influence the processes and outcomes of text-making ${ }^{271}$. She gives the example of Kress 2003, who suggested "that screen technologies prioritize spatial, as opposed to temporal, configurations of information - the image over the word" ${ }^{272}$. In some ways these theories lead away from strict SIP suggesting that with multimodality, rather than text alone, the mechanism have a greater effect on the non-verbal cues. She cites Labbo's 1996 on symbol making kindergarteners which suggests "that the computer enabled more freedom in the processes and products of the children's text-making" 273 . This is an interesting concept as it views CMC not as restricted but as a more flexible meaning-making space. Sakr's paper also suggests that mediation may play a higher role in emotional communication than is attributed to it in SIP. By looking at the ontological properties of the medium, Sakr problematizes the notion that text-based CMC is somehow less capable of creating meaning, suggesting instead that it may simply be that the output of text-based CMC is something incongruous with our previous understandings of text and self.

\footnotetext{
${ }^{270}$ Hunt et al 2012, 198

${ }^{271}$ Sakr 2012, 120

272 Sakr 2012, 120-121

${ }^{273}$ Sakr 2012, 120
} 
To a certain extent the debate over the implications of anonymity and identity in CMC is growing less relevant in an age where increasingly, communication online takes place through media containing carefully curated self-representations. Much of the work in CMC is focused on dyadic, personal text-based communication, but this is not the totality, nor the majority, of CMC. Anonymity was inextricably linked to many facets of early $\mathrm{CMC}$, but now it is an aspect that must be explicitly sought out. It is no longer the default. User profiles are increasingly specific and online relationships are more intricately tethered to those in the real world. Sites like Facebook began by cultivating friendships pre-existing the digital domain. Zizi Papacharissi 2012 suggests that the study of online communication is inextricably linked to our constructions of the self in the offline world. Users tend to have fractured, but connected versions of themselves presented online 274 . These may differ from site to site and platform to platform, as influenced by the site design. However, while they are suggestive, the architecture of SNSs are not necessarily inherently limiting. In fact it is mainly the social aspects of how other users make use of the site that shapes the performance of individual users within it. Papacharissi's article highlights some important aspects of how social factors influence the affordances of online communications technology. Papacharissi presents an interesting perspective on the performance of the self through social networking communication sites. Papacharissi suggests that because of the convergence of Communications Technologies through SNSs, users must combine several versions of themselves within a single frame of reference ${ }^{275}$. This is an interesting perspective on how the performance of the self, while varying from site to site, needs to converge alongside the disparate media. This understanding is helpful to creating a more nuanced picture of what computer mediated communication looked like for the experience of the

${ }^{274}$ Zizi Papacharissi 2012, 209

${ }^{275}$ Zizi Papacharissi 2012, 210 
JON. For example, on a site like Facebook where friends from various different contexts all access the same profile, so the user must make sure that it is accessible to everyone. For the JON this means that users must weigh not only their appearance to their peers within the Facebook group, but their appearance to their friends from their bowling team, their casual work friends, and that guy they talked to twice at lunch in high school. Alternatively they may be naïve as to how privacy works on SNSs and find themselves vulnerable to critique ${ }^{276}$. Support on a SNS can be a more public declaration of support in some ways, than attendance at an event. This is not based on the number of witnesses, but the number who recognize them and whose opinions they value (as opposed to a group of stranger). Here the reverse of anonymity is generative of boundaries (or opportunities) for the user.

From the preceding discussion we have traced the key issues in personality and 'self' expression that arise from the cue-constrained environment of CMC. On the one hand there is compelling evidence that anonymity can be generative of unique interpersonal connections and representations of 'self'. On the other there are instances where the SIDE model describes a deindividuating effect of anonymity that may cause greater affinity to groups than in FTF conditions. As the constraints shift with complicated understandings of online and offline worlds, some of the inbuilt characteristics of constraint in CMC become affordances for generating meaning and building identity. At the same time, what was learned from previous $\mathrm{CMC}$ with fewer modalities will still provide insight as the effects will likely remain, but become almost dangerously obfuscated.

\footnotetext{
276 Stephen Harper made headlines in 2011 when his staff 'barred' Facebook users from a political rally citing 'Facebook ties' to the opposition party in the form of photos on their Facebook page taken with Liberal Candidate Micheal Ignatieff (CTV.ca News Staff (April 5 2011)
} 


\subsection{Iteration}

In examining Digital communication, I expect to find that digital conversations are particularly segmented and iterative in order to ensure meaning transmission. In Face to Face (FTF) interaction most CMC theorists agree that frequency of feedback matters ${ }^{277}$. In digital communication, where mediated utterances are limited by affordances, feedback normally presented through non-verbal cues are adapted to suit the medium by users to ensure that understanding refers to intended meaning. This compensation in digital communication relies on segmentation and iteration. These occur during digital communications both because they have to and because they can. Byron 2008, Connell et al 2001, Choudury et al 2009, all attest that digital communication is less 'rich' than FTF communication ${ }^{278}$, but it also provides users tools that are unavailable in FTF communication. These tools fundamentally alter some of the conventional mechanisms that have adapted in analog communication creating entirely new strategies for communication using iteration and segmentation.

\subsubsection{Pragmatic and Emphatic Iteration}

There are two uses for iteration in digital conversation; pragmatic and emphatic. These are complicated by many factors, chief among them is time and the number of people involved in the conversation. Pragmatic iteration is when users ensure their meaning is understood in the correct perspective. This functions in many ways similarly to analogous communications technologies. Often it still relies on FTF or analogous forms of communications. However, evidence of iteration is clearly seen in its imprint in the digital world, even when processing has occurred beyond its scope. Day after day the users voice evolving opinions, or alter their style as feedback determines which strategies seem more or less effective at achieving their prescribed

${ }^{277}$ Byron 2008, 312; Hancock et al 2008, 295; also Walther 1996, 3-5

${ }^{278}$ Byron 2008, 311; Connell et al 2001, 118; Choudury et al 2009, 331 
goals. Within their utterances they gradually centre on the essence of what they are trying to express. The more iterations, the higher the likelihood of achieving shared meaning and effective communication. This is shown by Byron 2008, and Hancock et al 2008 who highlight the importance of rate of exchange $\mathrm{e}^{279}$. Communicators employ an iterative process that takes place both publicly (in person FTF, or using other analog private communication technologies) and through private digital and non-digital communications ${ }^{280}$. This kind of iteration is pragmatic because its aim is to ensure accuracy of meaning transmission; both sender and receiver share understanding.

Iteration can also be used emphatically to show importance of a detail. Iteration for emphasis can be seen in the act of "liking" sharing or "retweeting" certain utterances. It is also seen when a user repeats previously stated content. It is analogous to a crowd shouting a slogan, or a politician repeating key terms throughout a speech, but digitally it has important differences. The primary aim is emphasis and this is achieved by volume, but there is a kind of emphasis that is not possible in FTF and most analog communication; the extension of its temporal impact. Strate 2003 and Donath and Viegas 2002 discuss the impact of the history interface on digital communications technologies' interaction with time ${ }^{281}$. While retweets (the simplest example of this process) are nearly instantaneous for the sender, as the digital landscape changes, these appear not as a singular moment of noise (as with a chant), but a swell that grows to a peak and dies off. The impact will send ripples into the future. With the ability to see volumetric data such as number of "likes" this emphasis can be preserved and even expanded when 'real' voices would have long been silenced. The difference between this kind of archive and that of archived news footage is that the digital archive is available to anyone at any time (in theory). Iteration is

\footnotetext{
${ }^{279}$ Byron 2008, 312-313; Hancock et al 2008, 170-173

${ }^{280}$ See section 3.1 'Communication...' for definitions

${ }^{281}$ Donath and Viegas 2002, 364; Strate 2003, 367
} 
employed differently in the digital than in FTF because it also functions as memory ${ }^{282}$. There is a no standing record of analog communication, but there usually is with the history functionalities of most online communications interfaces. Depending on the amount of information given in each utterance, responses may not cover the points deemed important to the sender/poster. Repetition in digital communications is emphatic to show the importance of an idea and pragmatic to ensure understanding of the receiver.

\subsubsection{Segmentation}

The need for feedback also leads to the strategy of segmentation of important ideas into separate utterances, sometimes sent in separate streams. These streams can represent parallel locations of congress (outgoing), or separated channels of input (incoming). The former suggests strategic deployment of meaning tailored to the receivers of information at each location, and the affordances provided and expected across different platforms. The latter allows users to focus on and respond to individual segments of the message as they would in a FTF conversation. It is shown in the literature that speed of response can undercut reflection ${ }^{283}$, so it is often necessary to respond with different portions at different times. This even provides an option unavailable in FTF conversation, by layering time in a way difficult, if not impossible to reproduce with analog communications technology. Due to the ambivalence of synchronicity, this allows for several important aspects to be formulated and sent at the same time ${ }^{284}$. This is seen in the Journey of Nishiyuu by the fact that several conversations occur concurrently across different platforms based on temporal constraints. Separation defends against separate aspects being missed, ignored, or confounded as much public online discourse is not in the form of a typical back and

\footnotetext{
282 Donath and Viegas 2002, 363

${ }^{283}$ Henderson and Guiding 2004, 499; Orgura et al 2010, 3724

284 These ideas are explored in Kairos Chat (Orgura et al 2010 3723-3724) and by; Strate 2003, 367-369
} 
forth interchange. It is common for users in this way to carry on parallel conversations within the same conversation by separating them across different platforms, such as social media sites, or as responses to different posts or other centralizing 'artifacts ${ }^{285}$ on the same website. This is inherent to the idea of iteration, because ideas are expressed succinctly to be explored but tangents can also have a role as they naturally would. The ability to review historical comments allows users have assurance of understanding without sacrificing natural impulses to stray ${ }^{286}$.

Segmentation is not always a positive form of iteration because segmented information is not always reassembled in a shared context. While it allows for multimodality, it can also confound. With several conversations occurring on several social media and media platforms it is very difficult indeed to absorb the conversation in its entirety ${ }^{287}$. Thus information coming from any source will be definitively out of context, and so users rely on reiteration of contextual information to give it merit. A point which is uttered at only one location is less tenable than one which is repeated across many sites. While this is by no means a scientific form of knowledge creation, it does create a socially processed understanding of what the people within the conversation believe ${ }^{288}$.

\subsubsection{Hypothesis}

This paper aims to prove that iteration is inherent to the way users have adapted to digital communication. This leads to the hypothesis: Users will employ the strategies of pragmatic iteration, emphatic iteration, and segmentation throughout the conversation surrounding the JON as they discuss across various forms of digital communication.

\footnotetext{
${ }^{285}$ Choudry et al 2010, 8; Hansen et. al. 2010, 15

${ }^{286}$ Orgura et al 2010, 3724; Strate 2003, 372-373

${ }^{287}$ Dourish and Bell 2011, 25-27

${ }^{288}$ Walther et al 2005, 38, Hancock et al 2008, 295
} 


\section{Analysis of the Digital Conversation}

The following provides a detailed analysis of the digital conversation surrounding the JON. It begins with a detailed explication of the application of the methods employed in this study by outlining methods and conclusions drawn in detail. Next it draws the boundaries of the conversation and the study showing the constraints and describing why decisions were made to limit the study's scope. Finally, it outlines the evidence from the analysis of the JON's digital communications which supports the hypothesis that digital communication is iterative.

\subsection{Constraints of the Study}

It was necessary choose the breadth and depth at which to study the online imprint of the JON conversation. As a result, the aim of this study will be to provide examples of iterative communication in the context of the conversation, but only so far as individual examples provide new insights. To do this, it is not necessary to explore every avenue, so only select websites were looked at in detail. For the network map, categories were be made that encompass the totality of the discussion, but do not lose the key distinctions between the kinds of sites. There are also many aspects of the conversation surrounding the JON that will have been deleted by users, or exist in a different format today than they did when they were posted originally. This will be noted when possible, and assessed as to how it pertains to the development of the conversation, and the perspective from which it is studied.

\subsubsection{Form vs. Content}

It is necessary to make a distinction between studying the form of the conversation and the content of the conversation. From the Human Computer Interaction standpoint understanding how users interact with digital content is important, and this is affected by both form and content. 
However, it is not the particular interest of this study to become involved in the cultural and political effects of the protest being studied. More important to this project is observing how the form of the JON conversation (and by inference any conversation occurring in a similar form) develops and adapts itself to the digital platform. As a result, this study limits its scope to noting that a picture is a picture only in so far as the contents of the picture are important as formal aspects. For example if there is a photo of particular person, who that particular person is, is of less consequence than whether that particular person has a Facebook page that can be navigated to by clicking on their name in the photo. Likewise, a location in a photograph is important insofar as it is tagged and can be used to navigate to a digital map, or recognizable, such that the user can navigate the digital map or even in analog map on their own. Information gleaned from images can also provide context to statements surrounding it, or conversations adjacent to it. For example, user profile pictures can be indicative of their political persuasion within the conversation. The Twitter user @idlenomore4's profile picture is highly symbolic of the idle no more movement, thus the user name and profile picture frame @idlenomore4's comments as supportive of the movement ${ }^{289}$. For comments using text, the content of the text is relevant for its formal properties (i.e. whether they form a hyperlink or an emoticon) but also for its referents as they relate to the comments of others. Ultimately while there is a line drawn between form and content it is a blurry one. It is impossible to separate the two entirely when discussing meaning creation. As a result things like the tone of the comment as either positive or negative, or the framing of a post as supportive or adversarial are noted in reference to the effect that has on the shape of the resulting conversation. That is; content is important only for its effect on meaning creation. It is not the intent of the study to make judgments on the veracity of statements, or take

\footnotetext{
${ }^{289}$ See Appendix C.1: Figure 4
} 
sides within the issues' political debates. At the same time, in understanding how users process information that is often important to take this context into consideration.

\subsection{Visualizations}

The three visualizations described in the methods section can be found in Appendices A, $\mathrm{B}$, and $\mathrm{D}^{290}$. The following provides an outline of the application and analysis of these visualizations with examples from the JON conversation.

\subsubsection{Volumetrics and Timeline}

An attempt was made by this study to show some degree of the volume of digital activity surrounding the Journey of Nishiyuu. The idea was to create a timeline that showed the increase and decrease of digital activity around a specific period of time surrounding the event. In relation to the events on the ground, timelines were be created for both Facebook and Twitter. Twitter provides a very good platform for doing this kind of analysis, but recent changes to the API have made metadata more difficult to access for historical events. As a result, the hashtag \#idlenomore was chosen to be representative of the traffic surrounding the March $25^{\text {th }}$ protest date as the walkers were inspired by the idlenomore movement and many tweets associated with the walkers used the hashtag. As Idle no more is a bigger movement, the volumes dwarf those dealing with Nishiyuu alone, but there were also more resources tracking the hashtag in real time so the data from these third party sources was available after the fact. Ideally, by seeing the way this conversation took shape on the two different platforms, we would able to infer how the different affordances from the different platforms change the shape and longevity of the conversation as it pertains to the number of users involved, and the volume of their utterances. For the purposes of

\footnotetext{
${ }^{290}$ For Volumetric Timeline(s) see Appendix C.3; For Network Map see Appendix A; and for Pathway charts see Appendix B.
} 
this study, a time period was chosen that allowed for an equal amount of time occurring both before and after the arrival on Parliament Hill on the $25^{\text {th }}$ of March 2013. The ideal time period was from January $12^{\text {th }}$ to June $12^{\text {th }} 2013$. This period illuminates the development and growth of the digital conversation, and its lasting impact within media and social media after the main 'event'. In relying on third party data, a longitudinal chart was borrowed from a previous study that tracked the hashtag '\#idlenomore’ from January $1^{\text {st }}$ to March $30^{\text {th }} 2013^{291}$. From the comparison of the Twitter and Facebook timelines we can see some differences in the shape of the conversation on the different media over time. With Facebook we see a much smaller relative spike on the day of the protest. The number of posts on March 25 peaked at 276, compared to the previous day's 179 posts in the following day's 121 posts. On Twitter, the number of posts on March 25 was 9007, compared to the previous day's 2483, and the following day's 2961. The traffic throughout the period of the JON and beyond shows a flatter and more consistent trend for Facebook posts, when compared to the same visualization from Twitter. From the Twitter graph, we see a large period of activity in early January, the dies off toward the end of the month and remains with the comparatively low level of activity until the Nishiyuu protest. With the Facebook graph, there are fluctuations from day to day, but there is a relative consistency of posting before and after the culminating protest. Some of the explanation for this discrepancy can be attributed to the fact that \#idlenomore was established movement, with most of its protests centered in early January. The JON walkers had not yet left Whapmagoostui when the Facebook timeline begins. While connected to the idle no more movement, the Nishiyuu walkers headed to establish themselves as a distinct movement, being but seven walkers and one guide at the time of their departure on January 16, 2013. Also important to note, is the difference in kind of data used to create charts. For Twitter, all activity except 'favouriting' registers as a Tweet

${ }^{291}$ Gregoriev 2013 see Appendix C.3 Figure 30 
(this includes replies, and retweets). From the Facebook group, the data represents only individual posts, which means that comments posted as replies to previous posts not register on the chart. There are several posts on the Facebook page with hundreds and even thousands of replies ${ }^{292}$; data which is not represented on the chart. It could be suggested that this data would cause a greater surge on the day of the protest, but equally there are many replies to posts both before and after the protest which may retain the smoother curve.

\subsubsection{Network Map}

As a way to understand how the conversation surrounding the JON spread out across the online world, a network map was created showing the locations of conversation about JON in relation to the affordances provided at each location ${ }^{293}$. Hansen et al., the authors of the companion book to the NodeXL software used in the network map, suggest that "Social network analysis offers a systematic method to evaluate social media efforts" ${ }^{294}$. A straightforward social network analysis which would involve mapping the links between individual users contributing to the conversation. While this kind of analysis would be informative for some situations, in this study the focus is on how the mediation of the JON conversation affects meaning creation. Using a similar methodology to that which creates social network analysis, the NodeXL tool was used to create a map of the relationships between locations within the conversation surrounding the JON, and the media afforded by the different sites.

From the network map visualization we see a representation of the shape of the digital conversation as it might exist in a physical space. Each location within the Jon is represented by a square node, and each affordance provided by these locations is represented by a circular node.

\footnotetext{
292 see David Kawapit (March 26, 2013); Bob Mullins (March 14, 2013); Appendix C.1: Figures 12 and 14

${ }^{293}$ See Appendix "A"

${ }^{294}$ Hansen et al. 2011, 8
} 
Within the NodeXL program itself each node can be manipulated, and in dragging a node its connections become more evident. For the final network map, the circle format for Chart 1 was chosen as it provided the best two-dimensional view of all of the connections ${ }^{295}$. Other graphs were rendered using different clustering formulas ${ }^{296}$. This can show more clearly that certain sites are hubs for interconnections between locations or offshoots to the main body of the conversation. Facebook for example, is an important hub providing incoming and outgoing links as well as a greater number of affordances. Flickr, by contrast, is an offshoot, providing few affordances and mainly incoming links from select social media sites. Also seen is how other kinds of sites have a more internal focus. This is also evidenced by the pathway analysis. For example some media websites, do not necessarily encourage users to leave the site and look elsewhere for information. The chart also shows that there are common affordances shared across the sites, suggestive that the same digital objects can be found in multiple locations. The sites that contain internal linking which provide links between various locations within their domain are shown in the map with a location arrow looping back on itself. For example a user may navigate within CBC.ca through a hyperlink to another article from CBC.ca. Similarly, a photo on Facebook may provide a link to another group or user page within Facebook.

The interconnection between affordances and locations provided by this chart show much about the JON conversation. Also apparent are correlations between affordance richness and interconnectivity, and which affordances are most often available on different sites or types of sites. Hansen et al. highlight how "social media systems have often are evolved around a distinct type of digital object: videos at YouTube, photos at Flickr, [...] people at Facebook, tweets at

\footnotetext{
295 See Appendix A, Chart 1

${ }^{296}$ NodeXL creates clusters using an algorithm based on a Fruchterman-Reingold layout (Hansen et. al. 2011, 7.2.1); see Appendix A (Chart 2) For the Network graph generated with this clustered layout. NodeXL also allows for manual clustering layouts 7.2.2; (Hansen et. al. 2011, 7.2.3)
} 
Twitter, [etc.]"297. Ideally, this graph would be able to account for the volume of links and number of instances each affordance was used in the JON conversation, but this kind of analysis requires tools not yet created would be too great an undertaking for a conversation of this magnitude. This graph also shows how seekers information can find their way between the various digital locations in which conversations about the Journey of Nishiyuu are taking place through the linking available from shared affordances. Links between location nodes require direct linking available from the first location to the second (indicated by the directional arrows). As seen through the pathway analysis, these links are not available in every section of these locations, but this map shows the link as a binary between possible and not possible. The directionality is important because there are instances where the nature of a social media site means that it is possible to link from that site to other sites, but not the other way around. For example, the journey of Nishiyuu website (Nishiyuujourney.ca) was pinned onto the website Pinterest, creating a direct link from Pinterest to the site. However, the site itself contains links only to the social media sites of Facebook and Twitter. This means that users would have to manually pin the site to their Pinterest boards themselves. While every site on the Internet is technically connected to one another, they are not all experientially connected and navigation between sites is highly influenced by affordances ${ }^{298}$.

The network map provides an overview of the general shape of the conversation, which, while not holistic, gives a visual of the theoretical physical dimension which aid in understanding how the conversation takes shape in in an otherwise formless setting. Most importantly it does not restrict itself to individual social media or media sites, but shows the wider angle, highlighting the importance of analyzing conversations in the digital world and their

\footnotetext{
${ }^{297}$ Hansen et al. 2011, 15
}

${ }^{298}$ Hansen et al. 2011, 11 
existence in many locations simultaneously. From this perspective we can comprehend the effects of both the interface and media on access to information and communications affordances.

\subsubsection{Pathways}

From the analysis of the user pathways different media are seen to have different transmissive properties. The location of the media is also shown to impact how the user views the information, and where the information can take the user. Each pathway provides the experiential architecture for user navigating through information, actions, and locations. Exploring different posts, with unique affordances even within the same location brings the user to specific options for future directions. The pathway chart has been constrained to information, actions, and locations relevant to the JON, but show a depth of possibilities and constraints ${ }^{299}$.

The chart shows how actions of the user lead to access to more aspects of the conversation. In doing so they can either constrain or expand the user's opportunities for linking to more information. For example, if a user chooses to click on the user profile from a Facebook post a wealth of opportunities are afforded them. However merely liking to post does not add to the user's opportunities ${ }^{300}$. Also the level of interactivity afforded with any Facebook post is higher than that afforded by the same on Twitter. Because of the greater variety of formats for information stored on Facebook, the site requires a more complicated architecture for navigation. Also opportunities for communication exist at every level of the charts for social media sites, before the media websites, there are many pathways that provide information without providing the affordance for feedback. For example a video viewed on the $\mathrm{CBC}$ website, is not always

\footnotetext{
${ }^{299}$ See Appendix "B"

${ }^{300}$ See Appendix B.2
} 
connected to a comment section. With online radio broadcasts, comment sections are unavailable.

The pathway chart also show a trend when linking from site to site. For example, from a mainstream media website article users can browse more media about the JON (when available) internally, but no links are provided to external (competitive) media sites. In alternative media, and blogs, there were often links to different media providers' articles on the similar topic. From the perspective of meaning creation, the user consuming media and the latter format would have access to potentially more well-rounded perspective in having the ability to read multiple articles from multiple sources. The user reading media on CBC.ca, however, would come away only with the opinion of CBC.ca on the topic.

With many of the paths on the pathway charts created, the end of the pathway was the beginning of another ${ }^{301}$. This shows a structural reiteration whereby sites return or direct users to hubs of information or communications affordances encouraging further exploration or interaction. By design, this aims to keep users engaged with the site, and increases the potential they will stay on or return to the site.

The charts also show that how a media object is treated affects the opportunities for users to interact with the object and fellow users. For example if a snippet of a news article is provided directly on the social media site the user can interact with other users within the social media site using the communications media afforded ${ }^{302}$. The same article, existing as a hyperlink post still affords the user with the opportunity to like immediately, or comment directly, but it is much more likely that the user would link to the source of the article before doing so. Linking away

\footnotetext{
${ }^{301} \mathrm{Ibid}$; For example a path ending in a node containing ' $<$ loop $>$ ' refers to returning to a previous node, and many chains end by referring to the beginning of a pathway (i.e a node containing "[see personal Information]" etc.) found elsewhere in the chart, rather than being repeated to save space

${ }^{302}$ See Appendix B.2 "Embedded Media Post on Facebook"
} 
from the post, the user is met with a series of options provided not by the social media site, but by the source media site ${ }^{303}$. Importantly, the device used to access information plays a pivotal role in how this mediation occurs. Certain media are not supported in the mobile app of social media such as Twitter and Facebook.

On the desktop web for example, comments are automatically expanded under images on the Facebook page. This provides the user with immediate contextual feedback that is only available after expanding the same information on the mobile app. However, where the desktop web provides content of comments to be easily seen, the mobile web provides the quantity; and affordance not always available for Facebook comments on the web. On the desktop web, quantities are only available were posts have so much activity that requires expansion by the user (somewhere in the range of $>10$ posts, depending on the number of posts expanded) ${ }^{304}$. This means that user impressions of volume in reply to a Facebook post, on the desktop web are spatial (i.e. how much space the comments take on the page) rather than numerical (how many distinct comments). The spatial element is not effective on a mobile platform because of limitations on screen size. What is an experiential creator of meaning on the desktop web becomes a scientific one on mobile, or when quantity reaches certain volumes.

This affects not only communications affordances, but contextual information. If a photo is located on a user's personal website, but linked to social media site, in order to view the photo the user will be directed to the personal website which provides both a greater context for the image and a new location to explore for more information. Depending on the context of the website this kind of linking can both encourage and discourage the user from this kind of

\footnotetext{
${ }^{303}$ See Appendix B.2 "Embedded Media Post on Facebook -Snippet" and "Embedded Media Post on Facebook" Link to Source"

${ }^{304}$ For the post by David Kawapit there is no quantity of comments given, but as the replies to Bob Mullins' comment do not fit within a reasonable screen size, they are quantified when minimised, and a counter appears to show what portion of the whole are showing as expanded; See Appendix C.1: Figures 12 and 14
} 
exploration, depending on the individual's bias and corresponding bias of the location of the photo. The same picture embedded in website encourages the user towards more internal exploration of the original site, and as mentioned previously allows the user to use the conversational affordances of the site in which the picture was embedded. If this is a social media website for example, this will allow it a greater audience potentially than in individual's user website. If the website is a media website, the opportunity for conversation is equally available, but the kinds of responses and the diversity of users will vary depending on the context.

Analyzing these user pathways across multiple sites shows us how the communicative interactions are designed differently for different sites. Importantly, it also shows us how the user's experience of information is altered by this design.

\subsection{Iteration}

This paper contends that digital communication involves the strategy of iteration to convey meaning. The following will provide evidence of iterative strategies within the JON conversation online. Once establishing the existence of iterative strategies, it will show that iteration is necessary to digital communications. Further, it will be argued that its existence is specifically enabled by the digital medium.

\subsubsection{Evidence of Iteration}

In order to prove the digital, communications are inherently iterative it is necessary to show evidence of users employing iterative communication strategies. We can see iteration at work is by documenting how digital communication occurs across multiple platforms. Often the 
same user posting exists in multiple locations ${ }^{305}$. This is seen when the same news article, image, or video are shared by different users on social media platforms of their choice. Such images are remediated ${ }^{306}$ into blogs, or are linked with or referred to by other forms of media either internally or from different online locations. Similarly we can often see the same content available to users across multiple locations on the web ${ }^{307}$. Several combinations of this kind of iteration exist within the conversation around the Journey of Nishiyuu. The use of multiple online locations allows for a diversity of entry points for users new to the conversation to cast a wide net for potential supporters or consumers of information. On the $25^{\text {th }}$ there were 3,683 distinct users tweeting the \#idlenomore hashtag, each with their own sets of followers and friends. For example, articles, posts, and conversations about JON can be found on multiple news media websites, both mainstream (CBC, CTV, Globe and Mail, etc.) and alternative (Rabble.ca, Idlenomore.ca, iPolitics.ca, etc.); a wide range of social media sites including, but not limited to, Facebook, Twitter, YouTube, Imgur, Flickr, Tumblr, and Pinterest; and several personal blogs and independent websites (Nishiyuujourney.ca, ahki.co). For informed users the spread of the conversation onto multiple platforms allows for a multitude of loci in which to converse about or explore the topic.

Another kind of iteration is a form of segmentation where multiple users can be seen posting across, or consuming information from multiple locations across the web. A full explanation of the strategy of segmentation follows in section 3.4, but simple evidence of this can be seen in the fact that commenting on any news article posted to the Ottawa citizen requires login to users' Facebook accounts. The program Disqus is designed to easily add conversational

\footnotetext{
305 Disqus.com; OttawaCitizen.ca

${ }^{306}$ See remediation as discussed in Guillory 2010, 322

${ }^{307}$ See Appendix C.1 Figure 26; This tablet drawn image was posted on a blog, after previously having been posted on the Journey of Nishiyuu Facebook page
} 
affordances and provide the same interface in multiple locations so that the same users can converse in different online locations. Allowing users to reiterate their opinions across multiple platforms that only spreads the word further afield, but allows them to hone their opinions by expressing them in different ways is afforded by different locations. The existence of RSS feeds and news aggregators provide opportunities for users to interpolate information from multiple sites. This is a form of iterative consumption. Iteration often bears a similarity to research, where conclusions are reformed with added information, and then restated. Since the process of consuming information online can never be completed, users formulate conclusions based on the information that they have consumed and alter these conclusions as new information causes them to.

Another form of iteration that takes place within the context of digital communication is when the same ideas or content are voiced simultaneously or over time by multiple users. This occurs with many of the affordances provided by various communications media and social media to 'like' and 'share' the content of others. An individual user's photo or manipulated image can be shared and liked and reposted in a similar way to a news item. In this way the same content is re-voiced by multiple users each time for a slightly different purpose to a slightly different audience. For example, 'Barham's' post to the journey of Nishiyuu group seen in Figure 10 Was originally posted by Youth4Lakes ${ }^{308}$. The original caption/comment pertaining to the photo was written by the latter, but 'Barham' added their own comments when posting it to the JON page. Barham's post reiterates the content both by sharing it, and by altering its meaning with a new interpretation. This kind of iteration is pragmatic, aiming to provide new (more specific) context for the digital object. Even retweeting provides a kind of pragmatic iteration in that it provides a new context for the Tweet by way of an endorsement. The retweet is seen by

\footnotetext{
${ }^{308}$ See Appendix C (Figure 10); Barham (May 14 2013)
} 
followers and friends of the retweeter in the context of that user's established online profile, allowing for much more than 140 characters' worth of meaning to be gleaned from the statement. This kind of iteration also creates an emphatic effect to consumers, even if not intended in each utterance. For example, users who contribute to conversation thread on Facebook and directly share their activity on their newsfeed. In this way it is the mediation, not the user that creates the emphatic effect.

A similar kind of pragmatic iteration is practiced in the media, when news articles are amended or updated over time. This is often done with unfolding events, where being the first to break a story is important. A brief report is posted, but more details are added as events unfold and there is time to collect quotes and edit informed opinions. It is difficult to track the progress of these types of published news media as they rarely exist in their original form for long. Taking a historical look at an article that was published in this manner, will only provide the researcher with the final product, not the progression. However, with many breaking stories newsreaders are able to see this iterative process in action ${ }^{309}$. This also allows an arena for conventional print media to create the kind of online added value, described by Graham and Greenhill, by being able to update stories in between news cycles ${ }^{310}$.

Pragmatic iteration is also seen when previous news stories are pulled out of the archives to provide context for any developments that have occurred. Sometimes old footage or information is "republished" to provide background information for new events ${ }^{311}$. This can take the form of a hyperlink to an archived news piece, or a direct redistribution of old information with new information added. This is most often done by the people who publish the new story (in

\footnotetext{
${ }^{309}$ Paglia describes the process by which the online webzine Salon published articles "by stages" when the death of Princess Diana fell into a newspaper press vacuum of nearly 30 hours. (Paglia 2003, 275)

${ }^{310}$ Graham and Greenhill 2012, 91-92; 98

${ }^{311}$ For example, an article about the documentary created for the journey of Nishiyuu provides links to older articles from the time of the protest for context. Little, Jaime (Feb 11, 2014)
} 
whatever format) but it is not exclusively their arena. Sometimes it can be a commenter who provides a link to background information either within the source media website, or in the comments on a social media page to which the news article has been linked ${ }^{312}$. In the case where the article has been linked to social media site, the background information is not limited to previous news pieces from within the same media group ${ }^{313}$. This does not necessarily mean it provides an alternate opinion, however it would provide for a greater potential for incongruity.

We see in the imprint of digital conversations that occur in public forums one of the prime examples of iteration in digital communication. Most exchanges of ideas back and forth between the same two users or amongst a group of users require elements of clarification and reiteration ${ }^{314}$. In reading only one comment or post, we get less information than from an exchange. Ambiguity is stripped away as more layers are added. In 'David Kawapit's post alerting users to his interview on $\mathrm{CBC}$, many users replied in answer to a user's query about which channel Shaw direct was broadcasting it on. One user responded "CBC is different for each satellite provider !" To which the original poster wrote "yuh... lol, that's why i wrote shaw direct ... :P". One user wrote "Channel 20 in Chisisabi" and another wrote "Watching it now channel 502 bell”. Also the first responder reiterated his response by providing a hyperlink for a pdf of Shaw Direct's channel listing ${ }^{315}$. Here we see an example of a question being asked in later clarified, a user reiterating their answer, and multiple users responding to the same question and even anticipating other questions by providing information not ask for (the channel for Bell). This post is also interesting because it shows users organizing to watch event on live TV, posting

\footnotetext{
${ }^{312}$ For example a link is provided in @ 0 rabble.ca's tweet: “\#Nishiyuu is trending in Canada. Here's some background about this epic journey http://rabble.ca/news/2013/03/indigenous-youth-epic-journey-ottawa-deserveattention-and-respect ... \#IdleNoMore \#cdnpoli” (2013-03-25 12:22:00 PM) https://twitter.com/rabbleca/status/316268937505554434

${ }^{313}$ See Appendix C.1: Figure 11 for an example of similar linking within a Facebook post

314 See Appendix C.1: Figure 24; in their exchange 'Schlomo' and 'SkepticChris' show this in action.

315 Kawapit (March 24 2013) See Appendix C.1: Figure 12
} 
while watching the event, and posting comments about the event afterwards. Many commenters were also lamenting the fact that they could not seem to find a digital reiteration of the analog TV broadcast ${ }^{316}$. There seem to be demand for users using digital communications technology to view the event referenced on the same platform. Throughout the thread several users request that if anyone finds a link that they post it, an example of emphatic reiteration. Significantly, this shows an example of the fact that digital communications do not exist in a vacuum and are highly influenced by the events in the real world. When the link is posted, another commenter requested a summary "any summary anyone care to share? (Thoughts, words), if it seems appropriate." Feeling the need to later clarify by posting again "ps that were in the interview..." (Pertaining to the thoughts or words mentioned in the previous comment) ${ }^{317}$. This user, although no one takes her up on the offer, is asking for a reiteration of the live television event in the form of a Facebook comment. Throughout this post we can see how conversational digital communication requires pragmatic iteration to clarify exact meaning either to ensure meaning transmission, or to correct perceived errors in meaning transmission.

Conversations that take place in social media and other online forums can sometimes be no more than a one-way utterance of an individual user to an unseen, unnamed audience. However, even in the absence of a back and forth, post and reply format these are still iterative. They provide users with a crude volumetric understanding of what many people who read the article felt about it. For example, in an Ottawa Citizen article about the arrival of the walkers posted March $25^{\text {th }} 2013$, the first 22 comments are statements of support ${ }^{318}$. Posters either encouraged and congratulated the walkers, or wrote comments that were in solidarity with their

\footnotetext{
316 ibid

317 ibid

${ }^{318}$ Pearson (March 25, 2013)
} 
efforts $^{319}$. In this instance, the comments indicate, to the average user, that and most people are in accord with the article. This is a richer form of affirmation than we get from 'liking', but takes longer for the user to do and is not as easily quantified ${ }^{320}$. What we objectively learn from the comment section, is only that those who felt inclined to respond in the comments section had that opinion. This may have little to do with the majority opinion of those who read the article, and this is a very important distinction when considering how news items are construed differently based on how a user comes across them and whether the user is directed to the source website or not. Further discussion on this follows in section 3.4.

So far, this work has provided several examples of iterative communication within the JON conversation. However, evidence of existence does not prove it to be inherent to digital communication; for that, it must be shown to be necessary.

\subsubsection{The Necessity of Iteration}

Due to the poverty of nonverbal cues inherent in digital communication suggested by CMC theorists such as Walther 1997, Hancock et al 2008, Byron 2008, and Cheshin et al 2011, iteration is a necessary adaptation for users looking to make sense of information that is incomplete $^{321}$. Even with multimodal affordances such as videos and image based messages, digital interactions (specifically those provided in public forums, as opposed to private digital communications that would include things like synchronous video chats) lack many of the key elements that we rely on for precision when communicating with one another. This is explained

\footnotetext{
319 (Ibid.) One user began a thread instigating a conversation regarding the school curriculum from the sympathetic viewpoint but not enough is being done towards "righting the wrongs done to our First Nations" This comment had two replies, both providing sympathetic constructive, feedback.

${ }^{320}$ The number of 'likes' accompanies most utterances where 'liking' is an option for immediate numerical feedback. A user may not read very many supportive comments as they tend to be similar in theme and concept. ${ }^{321}$ see Walther et al 2005, 38-39; Hancock et al 2008, 295-296; Byron 2008, 312; Cheshin et al 2011, 3
} 
in the literature as being media-poor ${ }^{322}$. Information that provides clarity and re-assurance of understanding demands a negotiation. To express and understand the importance of information requires accentuation. As Walther 1996 and Henderson and Guilding 2004 show, understanding comes not from the utterances themselves but their context and their delivery ${ }^{323}$. So in the digital world, context and delivery come in a different format and require a different set of tools to be interpreted. Iteration is the foremost of these tools, and without it digital communication would not have evolved into the multifaceted and useful tool it is.

Iteration is such a necessary aspect of digital communication that it is interpolated on most platforms that provide any affordance for digital communication. Even Twitter, which at its foundation, according to Van Dijck, was a unidirectional messaging service that allowed utterances to go out with little thought about how they would be responded to, provides affordances for feedback ${ }^{324}$. For example, a tweet by @JianGhomeshi from March $25^{\text {th }}$ about his upcoming report on the JON received 10 replies, including a back and forth discussion between Jian and another user ${ }^{325}$. Analog newspapers had letters to the editor, which provided a highly controlled for of UGC. Editors saw so many letters and published so few that responses were polished by responders to increase chances of publication, similar to the process of academic writing. The responder knew they were writing for a single audience (the editor) and had only one chance to make themselves understood. Here we see an example of digital communication being more iterative than its analog counterpart. In a digital comments section, neither premise remains valid; the audience is plural and the opportunity for clarification and feedback is inbuilt. Nearly every article published online (even and especially blog posts) has its own section for

\footnotetext{
322 Primarily attributed to Daft and Lengel 1984 by Byron 2008, 329

${ }^{323}$ see Walther 1996, 7; Henderson and Guilding 2004, 490-491

${ }^{324}$ Van Dijck 2013, 224-225

${ }^{325}$ See Appendix C.1 Figure 7
} 
comments from readers, consistent with Landert and Jucker ${ }^{326}$. The prevalence of comments sections accompanying online publications is so commonplace that it is notable when it is lacking. This suggests that responding to publications (or seeing the responses of other's) is as important to experiencing them as reading the initial article. On the $\mathrm{CBC}$ website, an article that does not have commenting available notes that the commenting has been disabled for that particular article ${ }^{327}$. On the $\mathrm{CBC}$ website as with many of the media websites the comment section is mediated (in the sense that it is controlled) and express rules for conduct among commenters is outlined in their 'terms of use' section ${ }^{328}$. It might be argued that the pervasiveness of commenting was spurred on by the need for digital iterations of analog technology to provide an added value, but in looking at the picture of online news today the matter of fact manner in which commenting is treated shows how it is instantiated in the way that we understand online content. Equally, online news aggregators can be seen as an added affordance of the chat rooms that dominate the late 1990s early 2000s CMC scene. Movement in this direction is seen in the development of Chat Circles III's interface for users to gather around $\operatorname{media}^{329}$.

Social networking sites that began as platforms for users to build their own personal identities online, became loci for communication back and forth between users who negotiate the meaning of their various artifacts ${ }^{330}$. Essentially it is the ubiquity of affordances for iteration that prove its central role in digital communication. The Journey of Nishiyuu website does not have as its sole aim to be a site of two way communication, but it does provide for a comments section

\footnotetext{
${ }^{326}$ Landert and Jucker, 1423

327 See Dawn (April 13 2013); this article from the National Post disabled the commentary on an article that referred the JON because of the age of the article. Where the comments would have been was a bar stating "Comments for this section are now closed". This was likely due space concerns on the site.

${ }^{328}$ See CBC.ca (September 16, 2013) for specifics.

329 See Donath and Viegas 2003 provided their users with images to congregate around to stimulate discussions; also see Kaye and Johnson 2003

${ }^{330}$ Choudhury et al 2010,8
} 
below each blog entry and is linked to the Facebook and Twitter accounts associated with the walkers. It serves as a hub of information made official by its centrality to the protest, but it is its inability to ignore the need for negotiation of meaning that proves the necessity of iteration in digital communications ${ }^{331}$. The JON website's Blog posts were infrequent and have few comments $^{332}$. In order to keep the movement alive in the minds of its followers the site needed a venue that allowed for more traffic, and more exchange. As a result there are prominent links in the top left hand corner of the website to both the JON Facebook Group page and the Twitter account@Nishiiyuu.

Another essential function of iteration in digital communication is that it is relied upon for lay judgments in legitimacy. Given that users are more likely to accept something as true if it is corroborated by others, seeing multiple artifacts attesting to a given fact will increase the likelihood of acceptance ${ }^{333}$. For the consumer/receiver of information; this kind of iteration is pragmatic, expecting increased iteration to correlate with accuracy. There is a naïve understanding amongst many users that the visibility of items on the Internet has a correlation with volume $\mathrm{e}^{334}$. For instance a user that undertakes a search in any web search engine will expect that the results they received that are not demarcated as sponsored results in the order of frequency (and they would largely be correct, although most search engines do not disclose their filtering algorithms). The same inductive reasoning is applied to posts within social media, where this assumption is less likely to be accurate. ${ }^{335}$ If users see several people within their friend group iterate the same information it is afforded a higher probability of validity. When

\footnotetext{
${ }^{331}$ For more on hubs of interestingness see Choudhury et al 2009, 332, and 339

332 Nishiyuujourney.ca

${ }^{333}$ Kaplan and Haenlein 2010, 62; Bernstein et al 2013, 1-7

${ }^{334}$ Bernstein et al 2013, 3-4

${ }^{335}$ See Edwards 2012, 222; Facebook News feeds, for example, are tailored to the user based on their use of the site and their preferences. "The News Feed algorithm uses several factors to determine top stories, including the number of comments, who posted the story, and what type of post it is (ex: photo, video, status update, etc.)." See: Facebook (n.d) "Newsfeed"
} 
these assessments are made, it is not likely to be in light of the content filtering of the site. For example someone responding and interacting with certain types of posts will receive a heavier weight of the same in their feeds. This could lead to their mistaken conclusion that a greater percentage of their friends were posting these kinds of posts. In social media that use reposting as a form of sharing information, this can mean that a single influential user, when echoed by other users can create truth by volume. With the ability to browse between newspapers, or see a multitude of news broadcasts users can use similar tactics when assessing the legitimacy of news stories from news media as well. Users are able to tell if the same story has been picked up by several news groups. While each will report slightly differently on events, an informed reader will find points of commonality to be accepted as truth. Also, if the initial entry point to the topic is from an alternative media site, they may choose to search for references to the events reported by other media. When enacted by users, this form of iteration has an emphatic output. Here meaning is created by several users and sites independently and unknowingly providing legitimacy to each other's claims through an iterative process on behalf of the seeker of information.

One thread in particular brings up interesting issues surrounding the need for iteration online. A comment posted to a forum discussing a Yahoo! Canada News brief about Harper's decision to visit Pandas sparked a lengthy adversarial discussion about whether the walkers had undertaken their journey at all. The post by 'Terry' received 65 upvotes and only 22 downvotes, asked

Has anyone bothered to check this story out or is it phony like chief [Teresa Spence's] hunger strike. About 960 miles in approx. 65 days straight, no time off is about 14 miles a day. Where did they stay? Were any vehicles involved? Where did they eat the energy needed to travel those miles in cold weather would be a 
huge consideration. if they did it good for them, but if it was a scam like [Teresa Spence's] protest the readers should be told. ${ }^{336}$

'Terry' is obviously not a follower of the JON, but Terry's question about the legitimacy of the protest provides tangible evidence of the need for iteration when consuming digital communications media as well as when conversing. The article (at 350 words) was shorter by far than the comments section, and ended with a question aiming to spark discussion. It could be argued that this was less a news article than a conversation starter, and that Yahoo! News is more akin to a chat room than a news service. The Article contained two images, the most prominent of Harper greeting a Panda, and the smallest showed a group of walkers gathering "in Ottawa"337. The article did not expect to stand alone, it linked internally within the first three lines of text to a CBC.ca online video showing the protest ${ }^{338}$ with a much longer article providing context. This link in turn provided access to photos, videos and other related content from $\mathrm{CBC}$. However, with only the article to go on there is not much evidence to substantiate the JON and many users who may not have clicked the link(s) appear to agree with the skeptical Terry. One poster, 'Pickle', wrote "It undoubtably was a scam" and mentions not having seen any pictures showing the entire walk ${ }^{339}$. These users appears to be only consuming information from one, or possibly two sources and provide evidence of the necessity of pragmatic iteration in digital communication. Specifically in consuming digital communication, users rely on corroboration of stated facts in many formats (words, video, images etc.) the complexity of which would presumably be more difficult to stage.

\footnotetext{
336 See Appendix C.1: Figure 24 'Terry’ posting on Yahoo! Canada News (March 262013 )

337 Yahoo! Canada News (March 262013 )

${ }^{338}$ CBC.ca (March 25 2013) “Cree Walkers meet Minister at end of Idle No More Trek” Last Accessed April 4, 2014 URL: https://ca.news.yahoo.com/journey-nishiyuu-end-ottawa-morning-104314582.html

339 See Appendix C.1: Figure 24 'Pickle' posting on Yahoo! Canada News (March 262013 )
} 
As seen from the above, no individual piece of a puzzle can show a complete picture, and because digital communication relies on an iterative process that develops gradually over time cases like the JON cannot be encapsulated by a single artefact. In attempting to re-create the Journey of Nishiyuu using its imprint on digital communications media, it is clear that no individual utterance because of constraints of time, space, and affordances can provide a holistic account of the events on the ground. However, after immersing in the conversation a picture begins to form that provides a nuanced understanding. It is precisely through iteration that this increased understanding occurs. Essentially what we are looking at is the meaning created in the intertextuality of the online conversation. ${ }^{340}$ Not only more information, but information interacting with other information and refining itself iteratively is what provides this perspective. As a result, no individual post, without reference to other aspects of the conversation, either digital or otherwise, creates the same nuanced meaning as a series of related posts or utterances.

It has been shown above that iteration is a necessary process to allow for effective meaning creation in the digital world. Iteration is also something that is uniquely afforded by digital communications technologies. Users adapt to the affordances of digital communication and affordances are increasingly designed for these adaptations. This creates an iterative cycle which shows the inclination of digital communication towards iteration that feeds back into its necessity.

\subsubsection{Iteration Enabled}

The necessity of iteration to digital communication is part of the picture, but part of the reason that iteration is such a useful tool is because digital communication is uniquely suited to

\footnotetext{
340 "Intertextuality is the term [from discourse analysis] often employed to describe the assumption that meanings are produced as a series of relationships between texts rather than residing within the text itself" (Waitt 2010, 223)
} 
providing for it. No other communications technology is capable of providing for the kind of iteration that occurs in the digital world. No individual letter writing campaign can equal the volume of users who retweeted the star studded selfie Ellen DeGeneres took at the Oscars in $2014^{341}$. This may not speak to the figurative volume that a mass of humanity standing in protest of something creates, but it shows the power of the digital medium toward iteration. Some acts of iteration exist almost outside of space and time, in that they occupy an inconsequential amount of RAM, cognitive processing power, and time to execute.

One of the affordances for iteration in the digital world that has accelerated in recent years is the ease of sharing information. The mobility of online digital communication provided by smartphones and tablets allows users to interleave online experiences with daily life. 'Live tweets', 'podcasts', and 'live streaming' of events provide not only new tools for broadcasting, but also for consuming commentary on events. Supporters unable to attend the protest in Ottawa on March 15, could follow the live streaming link on a website to see the protest as it unfolded from the broadcaster's point of view ${ }^{342}$. It is not only those who post information from key locations during events on the ground, but co-located consumers of information. Protesters at the COP15 rally followed instructions via twitter for the kind of slogans that they shouted or held above their heads ${ }^{343}$. These are good examples of real-time sharing in the impact that accessibility to these tools can have on real events. Specifically, these show tools newly available with digital technology (real-time, mass-user communication). The frequency with which users return to sites of conversation, and the amount of time they spend conversing is relative to both

\footnotetext{
${ }^{341}$ Smith (March 3 2014); the tweet surpassed a photo posted by US president Barak Obama as the most Tweeted image on Twitter with nearly 3.5 million retweets

$342 @$ onthebeat1 and @ryanpaulgibson (March 25 2013); The live tweet from the CBC for the Journey of Nishiyuu can be found here: http://live.cbc.ca/Event/Journey_of_Nishiyuu_comes_to_an_end

${ }^{343}$ Sergerberg and Bennet, 206
} 
their level of engagement according to Choudury et al $2009^{344}$, and the availability of connections and communications device(s). Limitations are increasingly user based, rather than affordance based. Either users have yet to fully adapt to the opportunities of new tools, or the tools far exceed our limited capacities as intimated by Hayles ${ }^{345}$.

Private communications have long been portable, but the ability to post on public forums from public spaces is relatively new and growing. When engaged in an active event, especially one that takes place outdoors, there is a heavy reliance on easily portable communications devices to undertake this kind of activity. However, as the world transitions to more ubiquitous computing technology is not necessary that an attendee of a protest, or of any event, need forethought in order to have the tools at hand to participate in the protest through social media. In fact it is mostly an afterthought, because many people carry smart devices with them everywhere they go. Through this we see that increased participation online in this way is a result of the fact that we are capable of doing it, not necessarily the other way around. How cell phones and other portable media devices are used is negotiated daily by users and designers alike, and is not only necessity. Dourish and Bell and Hutchby believe that sometimes creativity or novelty drives our use of technology $y^{346}$. Constant interaction, regardless of location, is an example of iteration being enabled by the affordances of devices or sites. In this way people reiterate their opinions at multiple times and in multiple digital spaces simply because they can.

The impact of the ease of sharing information extends beyond co-locative sharing at the time of the event(s). Importantly information posted on public Web platforms is available at the leisure of the user who seeks it. It is frequently updated, these updates a part of the iterative

\footnotetext{
344 see Chaudhury et al 2009, 339

${ }^{345}$ Kathryn Hayles suggested that human attention was the limiting scarce resource rather than technology when dealing with information exchange. (Hayles 2012, 14)

346 Dourish and Bell, 134; Hutchby, 123
} 
process of meaning creation online, and so users are able to access up-to-the-minute information on the topics they seek, or the topics they stumble upon at their convenience. Many users of social media will check in on their accounts daily ${ }^{347}$ with avid users checking in more frequently at key times. Many of the more influential tweets during the protest were from users who contributed more than one opinion on that day, either related or unrelated to the protest ${ }^{348}$. Data from \#idlenomore shows that the volume of users tweeting more than doubled the number of sources of tweets on March $25^{\text {th }} 2013^{349}$.

Ease of sharing is also an important tool when considering the influx of information that is available to users on any given day through any given medium. Facebook newsfeeds are cluttered with the utterances of hundreds of friends (depending on the popularity of the user) and in order to make sense of the din users will likely not fully process most of what they see ${ }^{350}$. An endeavour that is looking to increase its visibility must acknowledge that the time spent contemplating a post is short, and must consider that the time it takes to share and spread that message must be equally short. Social media sites as Kaplan and Haenlein suggest, rely on user generated content ${ }^{351}$. If all of that content was completely original to each user the sites would not have the traffic they do today. Sharing, therefore, is an essential part of the model as it allows users to very quickly 'create' content that encourages other users to spend more time on the site. As a result there are several affordances on most social media sites that allow for users to reiterate content or posts provided by those they follow. These affordances are of particular interest to users or groups who wish to spread information or increase their visibility. This works

\footnotetext{
${ }^{347}$ Kaplan and Haenlein advise businesses looking to use social media to promote and control their branding to post to SM 1-2 times a day to stay visible and keep up with levels of consumption (Kaplan and Haenlein 2010, 67).

${ }^{348}$ See Appendix C.2: Figure 28

${ }^{349}$ Blevis (March 26, 2013)

${ }^{350}$ Hayles provided an example of this in the form of a reading comprehension test taken using 'hyper-reading' (Hayles 2012, 55-56)

${ }^{351}$ Kaplan and Haenlein 2010, 61
} 
especially well for interesting topics as users will unknowingly spread the content by interacting with it, which activates unseen algorithms for promoting trending topics that have higher traffic. When the average user 'likes' a digital artifact, the original intent is that they show their appreciation of the content ${ }^{352}$. A secondary consequence is that depending on how each user has configured their privacy settings, their activity and the content to which the activity pertained, is made visible to their friends, their friends of friends, or their followers depending on the site(s) they use. This consequence is not hidden, but it is not the direct intention of many users. For those who are looking to increase their visibility it is something to be celebrated when an item receives a certain number of 'likes'. While Bernstein et al show that these numbers are not directly related to the number of users who have actually seen the content, it is perceived by users as indicative of their visibility and status ${ }^{353}$. For example, the JON website posted on its blog to mark the occasion of their $30,000^{\text {th }}$ 'like' on Facebook. It is clear from the tone of the post that its author considers the act of 'liking' as analogous with both sharing and visibility by signing off encouraging readers to "[not] forget to continue to spread the word far and wide! (i)"354. This can be seen as a direct call for the kind of emphatic iteration only available online. Transmissive activity can be even further abstracted from intended activity by sites that track number of visits, or the volume of traffic seen by particular artifacts within their sites. For instance, twitter generates a list of trending hashtags based on their use which are provided up to the minute in the left column of the desktop twitter interface. These trending statistics are region specific, for instance on March 25 \#Nishiyuu was reported to be trending in Canada by a Twitter

\footnotetext{
352 One Facebook user suggested that they thought 'liking' just meant that you had read a post, not that you agreed with the content per se (See Appendix C.1: Figure 10 reply by 'David' 10:54pm). This also shows that meaning of affordances is still being negotiated.

${ }^{353}$ Bernstein et al. 2013, 3-4

${ }^{354}$ Nishiyuujourney.ca (March 72013 )
} 
user. ${ }^{355}$ No individual user, or group of users would be capable of intentionally creating that kind of notoriety, but as the tweets add up and are reposted and reiterated throughout the site there is a resulting increased popularity due to the increase of popularity. These kinds of background algorithms exist in all forms of social media and are largely unadvertised to the general population of users ${ }^{356}$. This is usually because this is proprietary information and part of the underlying architecture of the software, but also because these algorithms are exploited by the social media sites for the purposes of advertisements ${ }^{357}$. If they were to lose control of the ability to push certain kinds of information, it could be exploited by the advertisers alone circumventing the revenue stream for the site. This means, however, when a protest like the Journey of Nishiyuu attempts to further its agenda through the same means, but without 'jumping the queue' financially the process is further abstracted. It also means that what looks like organic development in the digital world may not be as untouched as expected. The power of social media to influence its users in this way is shown in Kramer's 2012 study where he looked at the spread of emotion in Facebook and prove that manipulation of the positive and negative tones of a user's updates could influence the positive and negative updates of friends ${ }^{358}$. The influence was minor, but the scale was not. If anything, though, this study does provide a model for the power of tools such as Facebook for users to influence, even passively, other users.

\footnotetext{
$355 @$ rabble.ca (March 25 2013) tweeted “\#Nishiyuu is trending in Canada. Here's some background about this epic journey http://rabble.ca/news/2013/03/indigenous-youth-epic-journey-ottawa-deserve-attention-and-respect ... \#IdleNoMore \#cdnpoli" (12:22:00 PM)

${ }^{356}$ Facebook News feeds, for example, are tailored to the user based on their use of the site and their preferences. They describe the algorithm as follows: "The News Feed algorithm uses several factors to determine top stories, including the number of comments, who posted the story, and what type of post it is (ex: photo, video, status update, etc.)." See: Facebook (n.d) "News Feeds"

357 Kaplan and Hanlein 2010, 64-65

${ }^{358}$ Kramer 2012, 769-770
} 
A unique aspect of digital technology, particularly with regards to iteration, is the reproducibility of events. This is described by Strate as a complication of cybertime ${ }^{359}$. Utterances on the internet have an ambiguous timescale. If a user is completely unexposed to particular conversational arc pertaining to events that are new to them the user could be immersed in the content in the same way five years after the event(s), as they would have been five minutes after ${ }^{360}$. The only difference is the knowledge of the user that the events are not occurring simultaneously and that new content is not being added as they read. Most posts are dated and time stamped, even to the second. On some sites this information is posted relationally to the current time. For example, a post may be time stamped with ' 12 seconds ago', or ' 3 hours ago', or '370 days ago'361. Often sites employing this model will use more specific time frames the shorter the time frame transitioning from seconds, to hours, to days, to years, providing less information at each interval. The two different ways of marking time provide users with different focuses. The first format puts comments in the context of their time in relation to the media article and real world events. The second changes the context of the temporal information to referring largely to the likelihood that the original commenter would still be checking in and able to respond to any questions or criticisms. Another format that is seen when marking time online is a mixture of both, like what is seen on Facebook. This provides the immediacy of the relational time for recent posts, but refreshes at the end of the day to provide specific date stamps for older posts.

Experientially public message forums are usually set up asynchronously. That is when an individual user is looking at a page, it does not update until the page is refreshed by the user,

\footnotetext{
${ }^{359}$ see Benjamin 1935, 2-4

360 Strate 2003, 378-380

361 The Globe and Mail comments section employs this method, which created problems in this case because posts over a year old were not accompanied by data showing the temporal relationships between posts; See Appendix C.1: Figure 23
} 
despite the fact that other users may be adding content ${ }^{362}$. This is not the case with all public forums, but most comment sections of news media sites, and social media sites are set up this way. The importance of this is that the experience of the user reading the comments is atemporal in the same way a novel or film can be atemporal as a result of its reproducibility. The same experience can be had at any time provided the user suspends their disbelief and spends time digging to find the origin of the thread. In this way we can see digital communications as iterative, because not only are they expressed repetitively, but they can be experienced repetitively as well. The conversation is experienced and re-experienced through the preservation of previous utterances. Users can review for content (pragmatic iteration) or absorb changing moods (emphatic iteration). The JON Facebook group page can be seen as one such repetitive event. The conversation on the page is still unfolding with new posts nearly every day, despite the fact that it is been a year since the walkers began their journey. With every new utterance in the conversation the event is reborn and reiterated as a platform for discussion. With the Facebook community page, though unassociated with the official Facebook page, the user can even see a timeline and retroactively look at posts from its creation until the present day. Reexperience is not unique to digital media, but there is a greater proportion of the artifacts which create the original conditions of an online event preserved. A good analogy can be found in an old comic book, whose advertisements for 1980s action figurines provide old phone numbers and addresses that cannot be reached. Looking back through an old Facebook page, these same advertisements are updated with new information and accurate links. This updating both preserves the experience and fundamentally changes it. New users looking at posts going back over a year ago are viewing content that was posted in a format now to iteration old. However,

${ }^{362}$ Social Media sites are largely asynchronous, with a veil of synchronicity in the form of refreshing content and notifications (Hansen at all 2011,20) 
the fact that the format has updated and remains the same across the site means that the older posts do not formally identify themselves as such. As a result, skimming over the date information, the user can find themselves immersed as if the conversation were contemporary.

Digital communication forums provide unique venues for mass-audience communication in unconventional ways. For example a tweet can reach a wider audience than a speech in front of a football stadium ${ }^{363}$. Digital media platforms provide many opportunities for one to many communication not dissimilar to the kind of communication provided by the mass communication media of the $20^{\text {th }}$ century (and still existing today). The key difference is the time it takes to produce and distribute the content being stripped away to nanoseconds (theoretically $^{364}$. There is also a decrease in the time it takes to produce the material in many cases. Certainly there is a reduction in production costs and the infrastructure to transmit the information to the audience, already existing in much of the first world, is no longer the responsibility of the broadcaster. We see some examples in the JON conversation of individuals taking part in the conversation who do not have the fame or popularity of established media personalities, but are still able to reach a wide audience and have their tweets, or their posts shared by multitudes of users ${ }^{365}$. While there remain inbuilt power dynamics ${ }^{366}$, the ability for these to be circumvented speaks to the power of digital communication as a vehicle for emphatic iteration

Conversely digital communications technology provide the affordance for conversations flowing upstream to the conventional one-to-many format. It is possible for many people to

\footnotetext{
363 Smith (March 3 2014); as mentioned earlier, Ellen's selfie tweet reached over one million retweets in less than 30 minutes.

364 Strate 2003, 366

365 See Appendix C.2: Figure 28

366 See Edwards 2012, 222; Atton 2003, 8
} 
voice the same or a similar opinion, even in the exact same words. Many people can also be seen acknowledging or responding to the utterances of a single person as in the case of high-profile Twitter users, or Facebook fan pages. While these kinds of interactions existed previously in the form of fan mail, or screaming crowds it is notable that now these interactions occur in public forums where any responses can be broadcast by the recipient to a wider net and it is more likely that people will find solidarity with those who share their opinions based on the similarities between their comments. For example, if we look at the tweets during the JON protest on March 25 we see several tweets by high profile media personalities being retweeted by many less prominent users. Even liking or recommending redistributes this form of emphatic iteration piecemeal to all social media users. These actions are easily performed on the digital platform, but it would be exceedingly difficult to recreate the same dissemination patterns organically. The affordances provided for iteration in digital communication create an environment where this adaptation to the "less rich" medium of communication feeds back into its own necessity. That is, the need for iteration enforces a pattern of iterative affordances, which create, as they are adapted to, increased ability for iterative communication and an increasing necessity for iteration in order to understand meaning. The fact that iteration can exist more fully in the digital world than in previous recorded media enforces the necessity of its existence in order to keep up with the social creation of meaning ${ }^{367}$. Both pragmatic and emphatic iteration were seen at work in the digital communications surrounding the JON. BY looking at the evidence from the case it can be seen that these iterative strategies exist in the JON conversation, are necessary aspects of digital communications, and are uniquely supported by the digital medium.

\footnotetext{
${ }^{367}$ Research from CMC has suggests that socially created meeting creates a kind of feedback loop of adaptation. This can be expressed as: Lack (inherent to CMC) - Design of Affordances - Adaptation to Medium and Adoption of Found Affordances - Need for Established Affordances - [Redesign of Affordances] see Section 3.5 or Byron 2008; Walther 1997; Hutchby 2001; Hancock et al 2008; Hayles 2012
} 


\subsection{Segmentation}

Within the digital conversation surrounding the JON a prominent aspect of iterative communication was the strategy of segmentation. This strategy allows users to cope with aspects of communications that have been complicated at the intersection with the digital world. Complex understandings of size, time, and location need to be navigated when communicating online. To do so users rely on their ability to segment their production and consumption of digital utterances across different platforms providing alternative tools for digital communications.

\subsubsection{Conversations}

Like any kind of conversation, digital conversations can take place in multiple locations at different times, and with different people. From the point of view of an individual user the development of an idea involves personal contemplation, private discussions, silently absorbing what others discuss, discussing in groups, and so on over periods of time. With the advent of digital communications technologies the difference is that some of these public forums, and some of these conversations occurring virtual as well as real spaces. One of the features of iteration in digital communication is the ability for users to choose locations in which to tease out different aspects of the conversation. Unlike in the real world (exclusive of digital technology), users can be in more than one place (virtually) at once. They can carry out synchronous private communications with more than one user at a time, and use pauses in the conversation to mull over information in public forums such as social media. Often the choice to voice different opinions on different platforms is based on the nature of the opinion and the platform and the sentiments of the user. 
In the same vein the user comments and conversations that you find on different websites have set differences based on their location. The comment section on an article linked to the JON Facebook page show mostly examples of solidarity messages with the cause ${ }^{368}$. The comment section below a similar article on the source media website shows examples of comments of a more adversarial nature. This is based on the likelihood that the users who encountered the locations for the same article would have done so for different purposes ${ }^{369}$. Commenters may also be less informed on the issues, as seen in the Yahoo! Canada comments section; for some that article may have been the first that they have read on the topic ${ }^{370}$. Visitors to the JON Facebook page, would probably have some background information that enable them to find the page in the first place. Also the page itself, evidenced by the pathways, holds a depth of contextual information. Someone visiting the JON website would be doing so purposefully and it would be considered a safe space for people with the same points of view. The population on the a mainstream media website would have a more diverse range of points of view and therefore may not be inclined to immediately agree with the walkers' point of view. As a result of this difference, or perceived difference users may feel more comfortable posting certain comments in certain venues. For example on the $\mathrm{CBC}$ website, while you are required to sign in with a username, your comments are not directly linked to your person. On the Facebook page any comments you make will be linked back to your Facebook account and visible to whomever can see your comments based on your privacy settings. Your name and profile are attached to your comments which would for the most part discourage blatant flaming ${ }^{371}$.

\footnotetext{
${ }^{368}$ See Appendix C.1: Figure 12

${ }^{369}$ See Appendix C.1: Figure 23

${ }^{370}$ See Appendix C.1: Figure 24 Yahoo! Canada News (March 26, 2013)

${ }^{371}$ Johnson, Cooper, and Chin 2009 suggest that flaming attacks spread rapidly in computer mediated communication because posters feel anonymous and insulated from punishment (Cheshin et al. 2011,3-4); Similarly Reinig et al. 1997 attribute flaming to feelings of anonymity allowed on the Internet which buffers the attackers from punishment and shame (Reinig et al. 1997, 55); see also Postmes et al. 1998
} 
Posting more anonymously also allows users to be insulated from personal criticisms for their opinions. For instance, someone who feels in solidarity with the JON movement but does not feel it is their place to say so may prefer to do so in an anonymous forum as opposed to attaching such statements to their Facebook profile or Twitter account. The level of conspicuousness can alter the comfort a user has with voicing their true opinions on the subject. As a result they may feel more comfortable negotiating their ideas in an anonymous forum before reiterating them in a more public digital forum with their name attached ${ }^{372}$ (or eventually in the real world).

The location of a media object in relation to the user's access point can also alter the amount of discussion surrounding it, and the ability of the user to access it fully. A user posted their blog post to the JON Facebook page, and the Blog post itself had only three comments, but the discussion below the Facebook post had seven comments ${ }^{373}$. Media objects that have been fully integrated into a social media site, such as Facebook, will alter the tone and discussion surrounding said media object. For example, a YouTube video that you watch within your newsfeed channels discussion of the video towards the Facebook page internally. The same video within a tweet on twitter that directs the user to YouTube in order to view the video may cause the user to comment using YouTube's commenting forum rather than replying to the original tweet. This not only affects commenting, but also the likelihood that the same video would be shared internally for example, within Facebook, or, if the user is conducted to the external website they may choose a different platform to share the video on. Most of the time there is also the option to view the media item at its source location, but sometimes that source location is unclear. This can lead users to question the veracity of information that they are

\footnotetext{
372 see Walther 1996, 6-7; Henderson and Guilding 2004, 498-499; Chan and Cheng 2004, 308
}

${ }^{373}$ Ottereyes (March 25 2013) [Facebook blog post]; Ottereyes (March 25 2013) [Blog Post] 
reading. Conversely, being directed to the source location can also lead users to question their acceptance of the content based on preconceived notions of the bias of any given site. These assumptions are not only important when moving from site to site, but also within a given site there is usually information about the poster, for example their user profile within a social media site.

Looking at the pathways ${ }^{374}$ we can see that Facebook and Twitter link directly to news articles on the sites that host them, allowing users to access the commenting functions of both the social media site and the source website. In the example of the Ottawa Citizen site, if directed there from Facebook, on a desktop the user will already be logged in and able to comment using Facebook. This is easier for the user and would increase the likelihood that they will comment as it takes out the interim step of having to sign in or sign up for a separate account. However, the barrier-free commenting may not draw their attention to the connection between the comment section and Facebook. Also a frequent user of a site like CBC.ca that requires sign in can set their computer to remember the password and remove the inconvenience of sign in every time eliminating the intervention (while potentially sacrificing privacy). On a mobile Facebook platform the user will not see the comment on the Social Media site before linking to the article, unless they actively seek them out. However using a desktop computer the comments of their friends and acquaintances that may colour their reading of the corresponding media are already expanded. Also, depending on the access platform, the media may have a snippet that users may read instead of continuing on to the article ${ }^{375}$. In that case they may feel able to interact directly without linking away.

\footnotetext{
374 Appendix B

${ }^{375}$ Palme et al, 3; They refer to this as s 'free riding' effect in news aggregators
} 
Users can segment their digital conversations across many locations. The preceding discussed some of the location and affordance based reasons users may choose to decide to converse in one location over another. However, segmentation is a particularly adept tool at dealing with a problem particular to digital media; how can we process the immense volume of information we encounter?

\subsubsection{Volumes/Scale}

Digital content is rarely viewed in a vacuum, and on social media sites one of the most consistent details provided for any digital artifact is the interaction it has received from other users. This may exist in the form of the number of likes, shares, recommendations, or views. These numbers can have an effect on the interpretation of the artifact is legitimate or tangential; important or superfluous. When users looking to increase visibility of their digital artifacts they will likely take care to place them in context in which they will seem more legitimate. The kinds of things that are liked and shared on different platforms can sometimes be media specific and while many platforms are multimodal some media may prove to be more transmissive on different sites. As a result of this aspects of the conversation surrounding the JON that surround different media objects will take place in different forms depending on how different media objects are treated in different locations. As seen from the network chart some social media sites are heavily weighted towards a few media affordances, choosing to specialize in particular media. Other sites provide more affordances for media, and serve as hubs to the specialist social media sites and media sites.

Segmentation is not only a strategy of users but also one built into the design of systems in order to make them function. Websites have a tendency to specialize in certain kinds of media, either stylistically to distinguish themselves from other sites, or simply because other sites 
provide better services for certain affordances and the inclusion of those affordances is better outsourced to provide the best quality. Also, bigger SMSs will provide redundant affordances so users can direct upload or share from other SMSs. This is done to bow to user preferences while at the same time attempting to encourage the upload of the internal media that retain and entertain users ${ }^{376}$. For example while user uploaded videos are available on Facebook, the majority of videos are sourced from YouTube and embedded into a Facebook page. When using Facebook on a computer, the user is able to view the video within the Facebook page without navigating away. However, if viewing from a mobile device the user is directed to either the YouTube website or the YouTube application to view the video. On other sites YouTube videos cannot be embedded and all videos contain links to the external site. Commenting is available on the Facebook post and YouTube ${ }^{377}$. Using this example we can see how a medium, in this case a video, can have transmissive properties that cause users to move from site to site and therefore from location to location within the conversation.

Similarly photographs or images and their encoding can carry similar transmissive properties. A tagged photograph provides information about and often a link to a user profile for the subject of the image. The image may also contain a geo-tag that links a user to a Google map of the location from which the picture was taken if that metadata is available. Images, like videos, can often be sourced at an alternate website. For instance on the social media website Pinterest, images can be viewed within the site, or clicking on the link can direct you to the source of the image and the website from which it originates. In the example of Pinterest the image chosen is the majority of the information provided to users of the site. A brief description and a small yet often overlooked comments section is all that accompanies the image. As a result

\footnotetext{
${ }^{376}$ See Appendix B.2 for the differences between uploaded and externally sourced media

377 YouTube had its own user accounts for commenting at the time of the protests, but has since been enveloped under the Google + umbrella.
} 
the images found on Pinterest (another similarly visual site) surrounding the journey of Nishiyuu rely more heavily on their visual components and style. In order to be effective, the image itself must be compelling as that is what is preserved and shared within Pinterest. On visually-based platforms, such as Pinterest or Flickr, despite the availability of commenting in text those items most likely shared will be those with a compelling visual component. The website linked to the image may never be navigated to by many of the users who come across the image. However, often images of snowshoe wearing walkers, such as the one that provides the banner for the JON's Facebook Group and Twitter pages, or the JON website's logo were accompanied with hashtag references, such as \#Nishiyuu which could indirectly lead users to sites that employed the hashtag to further the conversation ${ }^{378}$. It may also lead to recognition of the hashtag if it were seen in another context on another site.

On different sites there are different expectations of what kind of material will be shared. Some sites are heavily reliant on text based materials ${ }^{379}$. For instance Reddit features online articles simulating print media as its main staple. Flickr is a photo sharing website, and while there are affordances for comments and captions, it is fundamentally about images. Specifically it is about photographs, although it is not entirely used as such. YouTube is primarily used for hosting video, but it is also often used to host audio tracks with limited visual components, sometimes consisting only of a slideshow, and sometimes having only a single image while the audio plays. ${ }^{380}$ While it is possible to go against these trends the conventions of the site will likely dictate the success of an artifact to garner attention. These trends are established partially by sites looking to differentiate themselves, and users finding affordances they like more or less from site to site. Each user will develop their preferences for what media types they store and

\footnotetext{
${ }^{378}$ For example the logo for the JON webpage (See Appendix C.1: Figure 2) was also shared on Pinterest and Imgur. ${ }^{379}$ Hansen et al. 2011, 15

${ }^{380}$ CerAmony Band (July 242013 ) uploaded a tribute to the late Isaac Kawapit in the form of a musical slideshow
} 
share on which sites, and these choices further instantiate the site as being specialized for particular media or modes of interaction. This is yet another example of the combination of design and user determination. This specialization also enforces segmentation. Not only that, but it provides a mechanism by which users will later make choices about where to share or discuss different media. They will also learn from experience how to approach different topics with the different user groups that they have come to expect on different sites (or even on certain subsections of the same site). Some sites will be more combative, others intellectual, and some satirical. A simple example it the separation of group sites (like the JON group page) within Facebook to provide a space for likeminded users to 'congregate'.

Another important thing to note is that interaction is not the only indicator that an artifact has been viewed. ${ }^{381}$ There are certainly immeasurable incidences of lurking by the bulk of people who come across online artifacts. From this assumption we can extrapolate that based on the number of utterances in the public forums surrounding the JON, that the traffic through private digital communication and other forms of communication around the JON were even larger still. Part of the strategy of segmentation and digital communication is to use more synchronous, personal communication when depth and nuance are required ${ }^{382}$. Unfortunately these conversations are beyond the scope of this project, but we may speculate that this is why much of this conversation did not take place in the public forums.

The device also plays a key role in the transmissive properties, and the segmentation strategy. Mobile devices have a plethora of affordances and capabilities that conventional laptops or personal computers do not have, however they are also constrained technologically.

\footnotetext{
${ }^{381}$ A recent study from Facebook showed that likes did not necessarily correlate to the number of viewers who had seen a particular post, but that in fact post can be seen by as many as $84 \%$ of what is posted on Facebook, with liking activity providing a poor indication of visiability (Bernstein et al. 2013, 4-5)

382 See Byron 2008, 320
} 
Computers are unable, for the most part, to be ported in one's pocket to a protest for the purpose of taking pictures and uploading them directly to the Internet. However similarly smart phones are not capable of opening certain kinds of files, and do not provide very good interfaces for precise keystroke entries. Tweets and short posts, as well as on-location photographs are sometimes better done from a smartphone app as they allow greater access and integration with real world events. Viewing some videos, typing long posts, and commenting on news articles remain currently desktop/laptop specific. This is a kind of forced segmentation where design cannot overwrite certain constraints, so affordances are cut to streamline interactions with key functionalities. An interesting aspect of this is that given the influence of user determination on affordances, this segmentation creates an artificial divide in development.

\subsubsection{Time and Synchronicity}

The tendency towards separation is affected by the synchronicity of the interaction. This pertains to the medium', but the effect has more to do with availability and the relationship between users, given the ubiquity of computing power. Also because of the nature of segmentation it is likely that multiple kinds of mediation are navigated by a user in expressing or consuming information about the JON. Adaptation (based on both technology and use) to a more conversational tone challenges the binary of synchronous lasynchronous creating much more fluid categories. Some interfaces designed for specific kinds of communication struggle to manage these new kinds of synchronicity, whereas others provide affordances for it. Despite the constraints of digital communication, the specific talent it has for dealing with this ambiguous synchronicity can lead to it being preferred to other 'richer' communications media ${ }^{383}$. For

\footnotetext{
${ }^{383}$ Time is fractured and no longer a binary between synchronous and asynchronous because of the affordances of digital media (Strate 2003, 379-381); (Byron 2008, 311-312) also discusses this kind of channel choice.
} 
example, the capacity to preserve conversations in a usable form has a number of advantages for productivity, and even plays a role in social interactions. It is this capacity to preserve, that makes the conversation around the Journey of Nishiyuu possible, and also able to be studied in this manner.

One of the important aspects of iteration in modern digital communication is the history function provided by most communications interfaces. This allows for utterances to be shared but importantly it also allows for reflection, as discussed by Walther 1996, Henderson and Guilding, and Amchai-Hamburger and Furnham ${ }^{384}$. It also allows for more complicated ideas of time as shown by Hayles and Strate ${ }^{385}$. Most social media sites preserve utterances to be viewed at times of convenience, but in doing so also provide the ability for users to respond in their own time. This can allow them to craft a meaningful response and collect their thoughts. It can also allow them to respond instantly with unfiltered thoughts or ideas. With user generated content the user also has the ability to filter their own content and most of the time the ability to delete their own comments upon reflection. However, as new comments pile in and new posts are added, most utterances are swept away therefore most conversations remain unaltered largely by complacency.

Longevity is a key consideration when opting for medium of public digital communication. For example if it is important to get one's message out quickly, but not necessary that it have a lasting impact, a higher traffic site such as twitter would provide a good platform. The user's comment can be succinct and to the point, and importantly instantly visible. For example, there are many tweets on March 25 of logistical information as to the location of

\footnotetext{
384 Walther 1996, 6; Henderson and Guilding 2004, 490; Amchai-Hamburger and Furnham, 1035

385 Strate 2003, 361; Hayles 2012, 86
} 
the walkers and the timing of the protest ${ }^{386}$. The JON website provides an example of the opposite case, where the events of the walk are recorded for posterity with a clean uncluttered design directing users to read the filtered official commentary about the walk. Lower levels of user generated content and opportunity for interaction provide a more static environment in which to provide information. The information is therefore more stable over time. Social media sites provide higher interactivity, with greater fluidity and less ability to control information ${ }^{387}$. Information shifts as opinions and topics change, providing users access to a much less uniform consensus due to the greater propensity towards vacillation. Similarly news articles published about the events of the walk go into greater detail, as they are expected to be more deeply considered and long-lasting than 140 character tweet. With the ambiguity of time in the digital world consumption time does not always relate to reflection time. Also because of the interrelation of the digital world and the other aspects of human existence the imprint of reflection remains impossible to record. The same length of utterance may represent two entirely different amounts of time each user considered the problem. Equally, digital utterances can even be created faster than they can be consumed allowing that some users are fast at typing and may expel their thoughts directly to the screen without a filter. These utterances could conceivably take longer to read and process than to send. This is seen in many of the comments posted, negative or positive to the JON conversation. Generic statements of support posted in the comments of an Ottawa Citizen article may have been carefully worded or oral exertions meted out by quickly moving fingers. In one instance, the same user who hurled an insult in one

\footnotetext{
$386 @$ Nishiyuu (March 25 2013, 8:32AM)

${ }^{387}$ Kaplan and Haenlein suggest that companies can no longer control the information found about them on the web due to this change from website based static information, to social media based crowdsourced information; see Kaplan and Haenlein 2010, 59
} 
comment presented a considered rational argument in another ${ }^{388}$. However, there is not actually a way of measuring which the user took more time developing. They may have been thinking about their argument while typing the insult. Equally, the insult might have been the more considered where the argument was a typed out stream of consciousness. With specific time codes, we can see some evidence of reflection, but what may look like evidence of reflection, may also be poor typing skills or a trip to the kitchen for a snack.

One of the unique aspects of digital communication is that in experiencing an event unfolding in the digital world no perspective provides for a total experience. For example if immersed on an individual platform, or possibly tracking multiple platforms at the same time, consuming the information as it comes out in real time can prove too great a task for an individual user. For example, someone tracking the tweets on the day of the protest would become overwhelmed and unable to keep up without an analytics tool, which would fundamentally alter the experience. As a result, integral to the strategy of segmentation in dealing with digital communication, the user choses where, when, and how much to seek out and interact with information. These choices mean the user ultimately decides the text they create from the overwhelming amount of digital information.

${ }^{388}$ See Appendix C.1: Figure 24 


\section{Conclusions}

This work aimed to provide evidence supporting the hypothesis that: Users will employ the strategies of pragmatic iteration, emphatic iteration, and segmentation throughout the conversation surrounding the JON as they discuss across various forms of digital communication. To do this, this work drew on literature that provided the context for understanding communication and meaning creation as it intersected with the digital world and evolved to its current state. This work has provided examples of the use of iterative strategies throughout the digital conversation surrounding the JON; shown the necessity of these strategies for creating and expressing meaning using digital communication; and described their instantiation in the digital tools for communication. Having done this, there remains only to take stock of the implications of and opportunities for these ideas.

\subsection{Text, Text, and Text}

Linked to conceptions of communication is the idea of text. One way that we can understand the complicated experiences of users traversing digital communication is by expanding our idea of text. The concept of what constitutes a text is by no means a concrete one. Different media, philosophical, and disciplinary conceptions alter the scope and bounds of any notion of text. The naïve understanding of a 'text' is that of an individual written work, such as a book or a play. This is complicated by nuanced understandings of writing that according to theorists like Barthes include creating and performing, which broaden the category of 'text' to various different forms of art and utterance such as painting, film, etc. ${ }^{389}$. Within digital media, previously distinct forms of mediation are often used side-by-side creating multimodal 'texts' which Bolter endorses as solidifying the idea of a text being beyond the page ${ }^{390}$. While this

\footnotetext{
389 Barthes 1975, 12-13

${ }^{390}$ Bolter 1991, 45-61
} 
understanding helps to clarify what constitutes a text, it further complicates how we understand the boundaries of what 'text' entails. It is not only the content of a text that problematizes its definition, but also the circumstances of its creation and interpretation.

Roland Barthes in his book From Work to Text distinguishes between the output of an individual author and the range of meanings created by the adaptation, interpretation, and greater understanding of the same. The former he refers to as a 'work', and the latter, larger category of

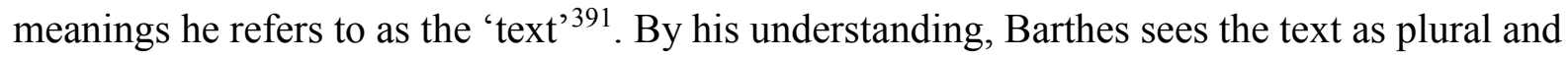
unending. He refers to the idea of intertextuality claiming that it is impossible to live outside of the text ${ }^{392}$. Bauldrillard describes a chaotic oversignification as being 'gorged with meaning' ${ }^{393}$. Barthes is attempts to allow an outlet for us from this state. He argues for a layering of significance within modern text that causes interpreters to graze rather than gorge on meaning, collecting bits and pieces of information that are then woven into their broader understandings ${ }^{394}$. Barthes ideas, while they predate digital communications as we know them today, can be seen to describe them quite adeptly. His idea of intertextuality very well describes the common experience of being unable to consume all of the available information on a topic, yet reaching a critical mass of understanding where one feels informed, while at the same time fully aware of the impossibility of being perfectly informed. Any initiate into the JON would have experienced this kind of self-informing balancing act, for even as I complete my project I know I have not read every post on the subject and can be fairly certain no one else has. Still, many will feel well versed in the intricacies of the conversation, despite the lack. Barthes' understanding of text also

\footnotetext{
${ }^{391}$ Barthes 1975, 37

392 Barthes 1975, 36

${ }^{393}$ His words provide an outline of a common criticism of the digital age: "Everywhere one seeks to produce meaning, to make the world signified, to render it visible. We are not, however, the danger of lacking meaning; quite to the contrary, we are gorged with meaning and it is killing us. As more and more things are falling into the abyss of meaning, they have retained less and less of the charm of appearances." (Baudrillard 1988, 63)

${ }^{394}$ Barthes $1975,12-13$
} 
allows for a site of meaning creation like that of the Internet that has many authors and many voices putting together of quasi-unified body of meaning. Constraining the idea of a text to a more conventional understanding, similar to what Barthes describes as a work (a bounded output of an individual) limits the way that we can understand meaning as created by multiple users in multiple locations at multiple times in tandem as a meaningful output.

These difficulties are further confounded by the electronic medium, which is itself in flux. Bolter's pseudo-definition of electronic text provides a good starting point from which to understand digital text. Bolter states that "...a true electronic text is not a fixed sequence of letters, but is instead from the writer's point of view a network of verbal elements and from the reader's point of view the texture of possible readings. An electronic text permits the reader to share in the dynamic process of writing. The text is realized by the reader in the act of reading" ${ }^{395}$. From this understanding, 'text' is not defined by any individual elements, but by the user's experience of creation and interpretation. These experiences are unique and multiple: "The reader calls forth his or her own text out of the network, and each such text belongs to one reader and one particular act of reading" 396 . For Bolter, as for Barthes, the text is never completed as it continues to be worked on by everyone who experiences it ${ }^{397}$. Bolter's understanding differs from Barthes' in the way they view the subjectivity of text. For Barthes, meaning is either created in the form of work, which is done by an individual, or a text which is contributed to and experienced by multitude 398 . For Bolter, each individual user creates a unique text based on their experience. Importantly, the text is not limited to words, but includes also multimedia, and structural elements. So, the text created by those who experience the Journey of Nishiyuu is

\footnotetext{
395 Bolter 1991, 5-6

${ }^{396}$ Bolter 1991, 6

397 Bolter 1991, 239

${ }^{398}$ It can never be truly experienced by any individual as it remains a relatively abstract totality
} 
unique to each experience, and each user. This provides a philosophical groundwork for navigating the mess and complexity inherent to the forum of iterative communication.

\subsection{Future Directions $\backslash$ Further Opportunities for Study}

There are many kinds of analysis that could be done using similar methods with greater resources, the ability to do a more detailed analysis of the same subject, or cover a similar subject on a greater scale. In an ideal case, it would be possible to provide volumetric timelines from all the forms of social media and media that exist throughout the conversation. Volumes could be added to the network graph to show not only how the connections take place but how often the user goes from one site to another and through which affordances. Similarly volumes could be added to show the frequency that affordances available (or used) on websites, so rather than noting that they exist or do not exist as a binary, we could see a detailed account of how frequently certain affordances are used and even possibly for which purposes. Ideally the network graph could be broken down to specific users within the networks that are communicating with each other. More realistically, being more specific about the kinds of media sites could allow for more nuanced understandings. For instance, an individual site like CBC.ca would be identified (potentially broken into component parts) as opposed to simply the generic category of 'mainstream media' or 'alternative media'. Tracking down every single website that mentions or refers to the JON was beyond the scope of this project, but certainly much could be learned from analyzing its incursion into the web beyond social media and provide some analytics as to how, and importantly, when this spread occurred. There are several analytical tools available for analyzing and visualizing data about the use of particular brands or terms within specific social media sites, but these tools rarely provide affordances for looking across platforms. There is even less available for tracking this kind of data across a multitude of 
websites that do not necessarily have that analytical data themselves. Also, where these tools are available, often the data is abstracted from its source(s) and detailed, qualitative analysis is not available to pair with basic visualizations. Despite the narrow scope of this project there is much that can be learned from this kind of analysis. More still could be learned from increasingly detailed analyses. The process by which these analyses may be undertaken would be a fruitful enterprise for other forms of studying the use of information, data, and digital communication. As we adapt to these new ways of using the Internet for communication and as a social environment, how our information and communication methods are manipulated by their mediation becomes increasingly important to understand. Understanding the mechanisms of digital communication and the new ways that meaning is generated through the ever evolving digital tools of the day is a daunting, yet important project.

\subsection{Summary}

Throughout this work, the conversation surrounding the JON was used to provide a tangible application of theory surrounding digital communications. In the JON's digital imprint we see how sites are designed around users' experiential pathways through information and that multiple paths are available. Users could access the same information 'in' different locations and from different locations depending on how they sought it out. We also see that the interfaces by which JON information is accessed change while the original content remains.

Examples were shown throughout the JON's digital conversation of the impact of communication technologies on the type and tone of the information shared. This demonstrates the effect of mediation, but also the impact of social constructs. Offline power structures were seen reified in the JON online conversation. This is an area which could benefit from continued exploration. Particularly, examining projects with differing levels of political charge could bring 
to light some of the inbuilt power dynamics of digital tools and perhaps highlight ways of overturning them. Also seen were examples of technology interpolating into the real world, as mobile devices took the digital conversation on the ground with the JON. From this we can see that it is not only technological development that will increase ubiquity in computing. Similar ends can be met by reframing our understanding of how technology interacts with the world, its users, and vice versa.

Despite remaining in the public domain this analysis of the conversation surrounding the JON found instances where the distinctions between the public and private spheres were unclear. While there were no catastrophic implications in this case there was evidence of the opportunity for user insecurity in the blurring of these lines. The JON conversation example also solidifies mass media as a combination of both volume and time in the digital world. It is not only the number of people who view a certain artifact, but that they do it in a shared timeframe that is important. In the early stages of the JON, it was social media that carried the momentum. This highlighted the importance of UGC and the transition from traditional producers and consumers towards more nuanced distinctions. Mainstream media was still influential in amplifying the message of the JON, but a critical mass large enough to be rendered newsworthy was created using social media ironically citing often the lack of interest from the mainstream media as a rallying cry. The renegotiation of the boundaries within the domains of digital and social media create new meaning making spaces that were utilized within the conversation surrounding the JON.

The JON provide several examples of individuals using communication technologies as they were designed and highlight how those designs enable the distribution of information. The JON shows the impact of instantiated design strategies such as history interfaces, user profiles, 
and linking or embedding content. JON also shows how use, as well as design, can be generative of affordances. For example, Facebook group sites were designed to allow for multiuser forums, but as the JON website became crowded with posts, threads within the group site became internal multiuser forums containing thousands of comments and replies.

The expression and perception of emotional content throughout the JON shows the adaptation of users to CMC. The effects of anonymity are shown to affect the kind of posts found in comment sections, also interpersonal relationships are seen in the lasting activity of the JON Facebook group which remains active to the present day. The ways users present themselves online was seen to affect the kinds of comments they were inclined to publish on different locations within the conversation surrounding the JON. In locations where self-presentations were converging (tying multiple groups together that would be disparate in the real world) statements tended to conform to norms or be absent. Where self-presentations were fragmented (specific to a particular site, or group) the tone of expression matched the location of the conversation.

These observations prove the hypothesis that digital communication is inherently iterative. The conversation surrounding the JON showed the use of pragmatic iteration as a tool for users to better understand the meaning of others, and elucidate their own utterances. Emphatic iteration was used as an apparatus to accentuate, or assess the importance of information. As the distinction between pragmatic and emphatic iteration remains contingent on user intent, further work would be required in distinguishing the volumes of each within the conversation. The JON digital conversation provided evidence of iteration in digital communication, showing that it is a necessary adaptation which is uniquely enabled in the medium. 
The conversation surrounding the JON spread across multiple platforms and channels showing users managing information via the strategy of segmentation. Conversations within the JON take place across multiple platforms, depending on a variety of factors ranging from site design to media preferences. The size and transmissibility of information or media affected why artifacts and utterances may have been found in different places within the JON. Also, segmentation was a strategy for providing different levels of synchronicity, when either the technology (ie. Mobile phones and platforms) or the user require it. However, segmentation also presents a problem for understanding how users process information they receive and distribute piecemeal. This is where the kind of is experiential text formation suggested in the discussion of Bolter and Barthes is helpful. Iteration is both generative of information overload, and a strategy for overcoming it. One of the consequences of suggesting that digital communication is iterative is that its adaptive strategies are reflexive. Any conclusion to this work would be incomplete without referring to the fact that in order to fully understand this complex and changeable process central to how and why we use computing technology in the first place we must continue to study it as it exists and as its existence in new forms challenges our previously developed understandings. While it may be tempting to suggest that digital communication strategies vastly differ from those communication strategies that preceded them, it may be more accurate to say that the concept of communication is itself iteratively refined and redefined through use and reuse. 


\section{Appendix "A" Network Map}

Location and Affordance Network

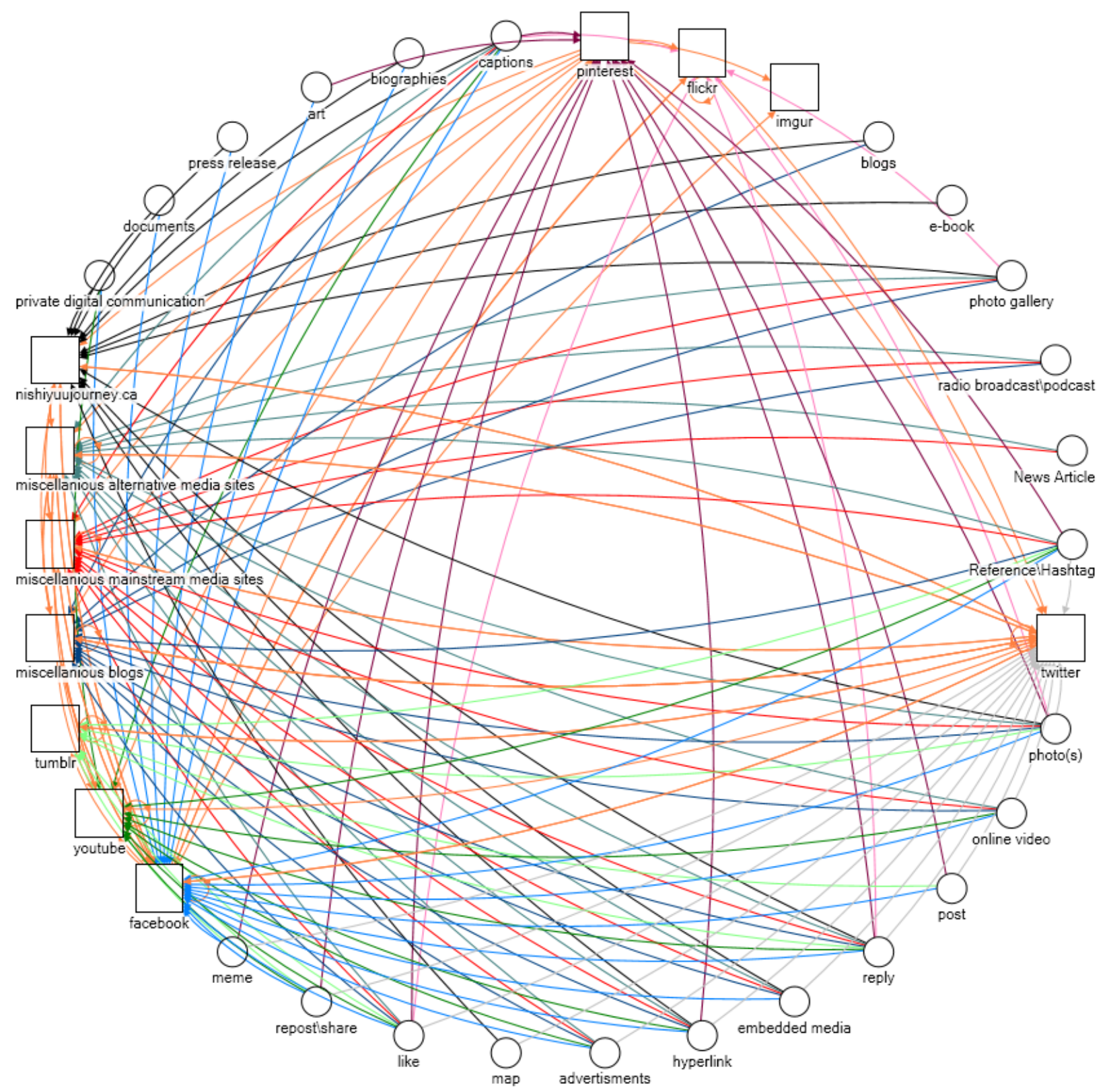

Created with NodeXL (http//nodexl.codeplex.com)

Chart 1: Network Map with circular layout for visibility 


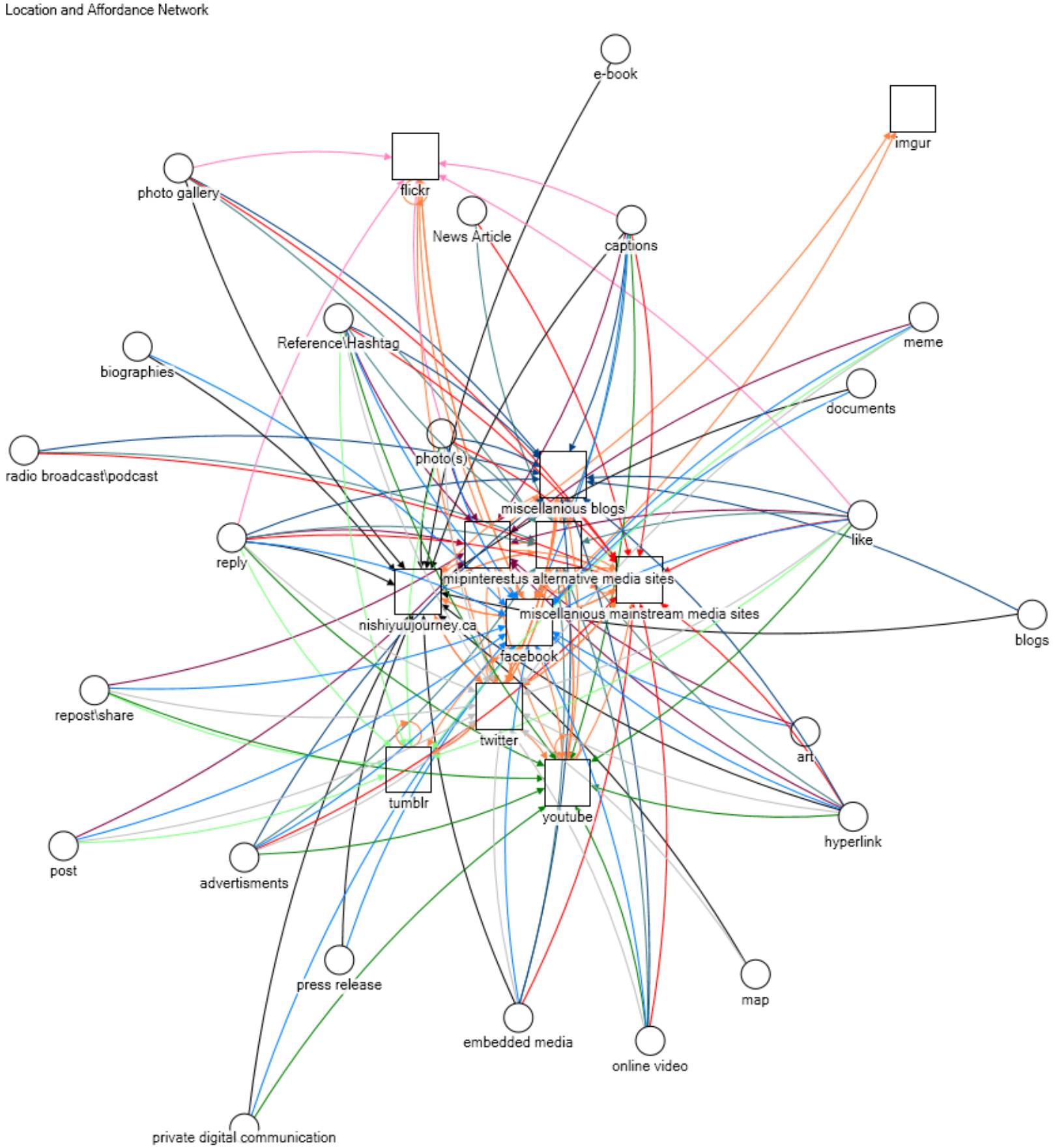

Created with NodeXL (http.//nodexl.codeplex.com)

Chart 2. Network map showing a clustered layout. Clusters were created using an algorithm based on a Fruchterman-Reingold layout (Hansen et al. 2011) 


\section{Appendix "B" Pathway Charts}

\section{B.1. Pathway Chart for Twitter}

Entry Points:

Twitter User

- "favourite" post

- Retweet

- User stream (subscribed)

Twitter Feed, via followed users, random generation, latest post

Twitter Search

Embedded feed (external)

"Trending" link

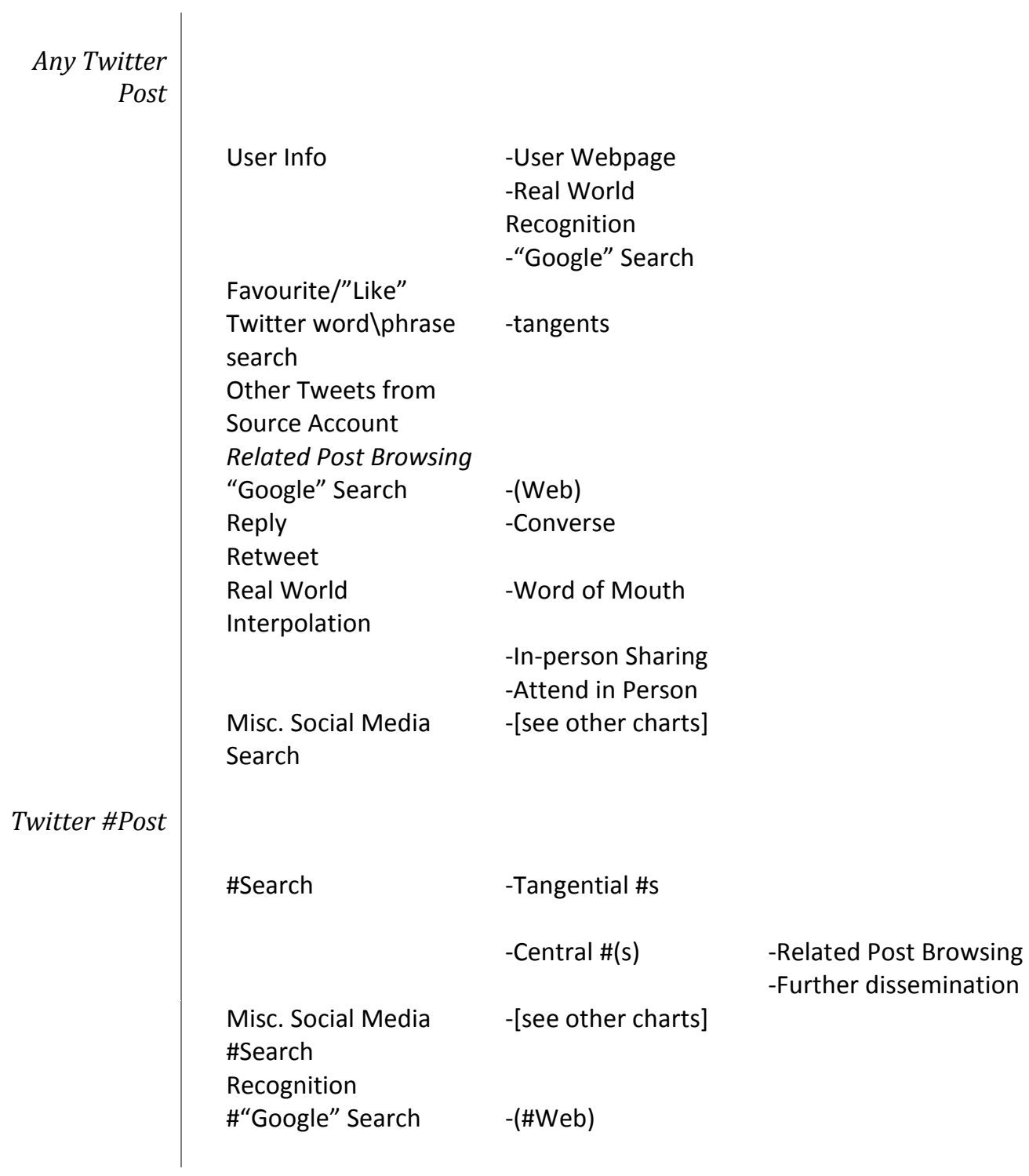




$\begin{array}{llll}\text { Real World Relation } & \text {-Place(s) } & \text {-Maps (Digital or Analog) } & \\ & & \text {-Attend in Person } & \\ & \text {-Person(s) } & \text {-SM connections } & \\ & & \text {-Private Communications } & \\ & & \text { (Digital or Analog) } & \\ \text { Source Link } & \text {-Photo SM } & \text {-[see other charts] } & \\ & \text {-Abstract Source (i.e. } & \text {-"Google" Search } & \text {-(Web) } \\ & \text { Fbook post photo) } & & \\ & & \text {-Misc. Social Media } & \text {-[see other } \\ & & \text { \#Search } & \text { charts] }\end{array}$

Source Link

-Misc. Blogs

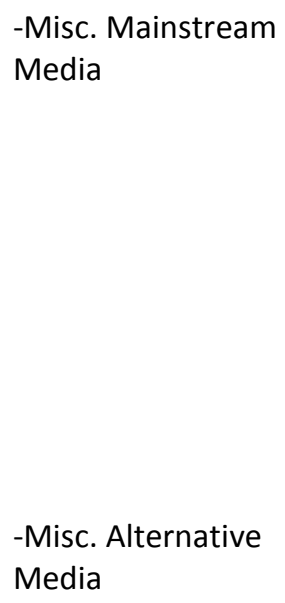

-Misc. Alternative Media

-[see all Twitter Posts]
\#Search

\section{-"Author Info" \\ -More media on same topic \\ -different topics on same site [tangents] \\ Follow on Twitter}

-[see user

info]

-"like"

-Reply/

Comment

-Share on

Misc. SM

-More media on same

topic

-"Author Info"

-different topics on same site [tangents]

-link to different like- -loop

minded Media

Follow on Twitter

-More media on same topic

-"Author Info"

-different topics on same

site [tangents]

-link to different like- - -loop

minded Media

Follow on Twitter -[see all

Twitter Posts]

-[see same in

MSM]

-[see all

Twitter Posts]

-[see same in

MSM]

-[see all

Twitter Posts] 


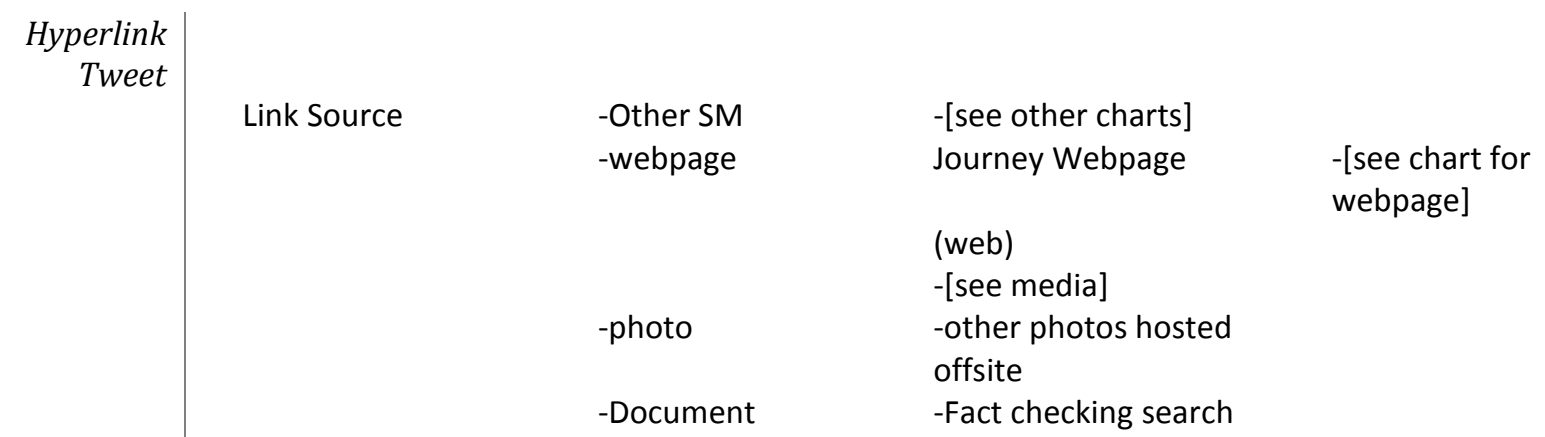

\section{B.2. Pathway Chart for Facebook}

Entry Points:

News Feed, via "like", Share, or Post

Embedded feed (on external site)

Link from webpage

User Timeline

Community Timeline (via search or invitation to "like")

Group Page (via search or invitation to join)

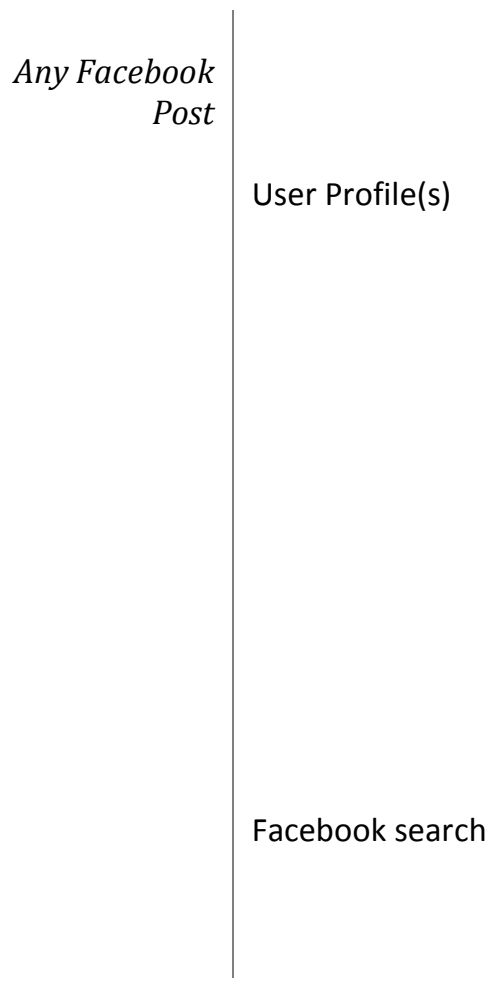

$\begin{array}{lll}\text {-Associated Group } & \text {-Related posts } & \\ \text { profile } & & \\ & \text {-tangents } & \\ & \text {-Other group members } & \text {-[see User } \\ & & \text { Profiles] } \\ \text {-Real World } & \text {-[see group information] } & \\ \text { Recognition } & & \\ \text {-friend } & & \\ & \text {-unrelated posts } & \\ & \text {-[see Personal } & \\ & \text { information] } & \\ \text {-not friend } & \text {-Related posts } & \text {-loop } \\ & \text {-open profile } & \text {-[see } \\ & & \text { "friend"] } \\ \text {-Facebook Groups } & \text {-private profile } & \\ \text {-Facebook Pages } & \text {-users } & \text {-[see user } \\ & & \text { Profile(s)] } \\ & \text {-communities } & \text {-[see user }\end{array}$




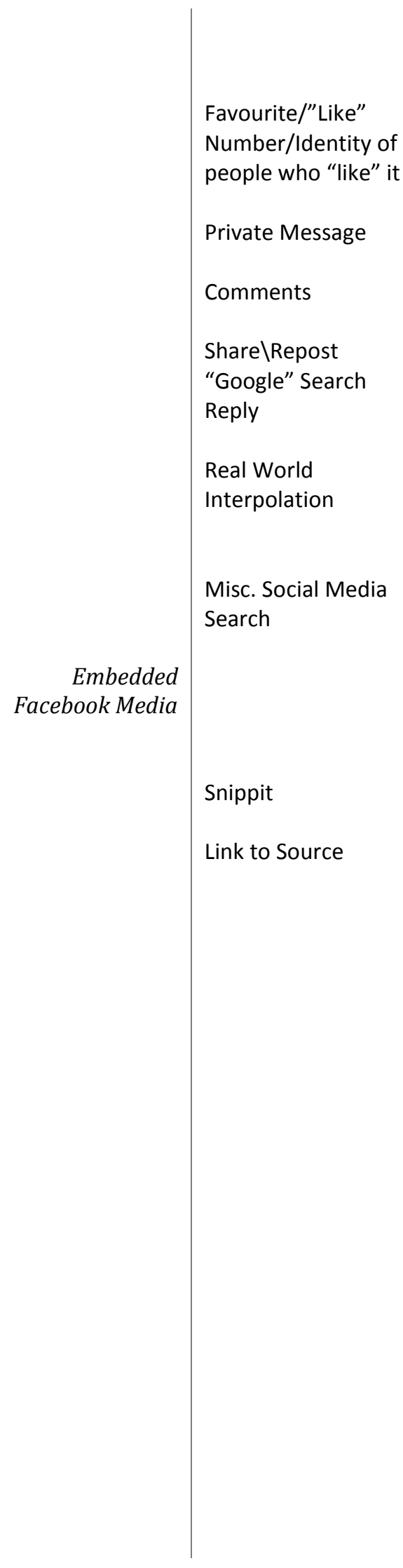

-Different with (web)
Graph search

Profile(s)]

-[see user Profile(s)

-gravitas

-Group

-individual

-\{see user profile]

-reply

-comment

-(Web)

-Converse

-Word of Mouth

-In-person Sharing

-Attend in Person

-[see other charts]

-converse

-converse

-converse

-converse

-reply

-converse

-Misc. Mainstream - "Author Info"

Media

-More media on same

-"like"

topic

-Reply/

Comment

-Share on

Misc. SM

-different topics on

same site [tangents]

Facebook page

-[see user

Profile(s)]

-Misc. Alternative

-More media on same -[see same in Media

topic

MSM]

-"Author Info" -different topics on same site [tangents]

-link to different likeminded Media

Facebook page

-More media on same topic -[see same in MSM] 


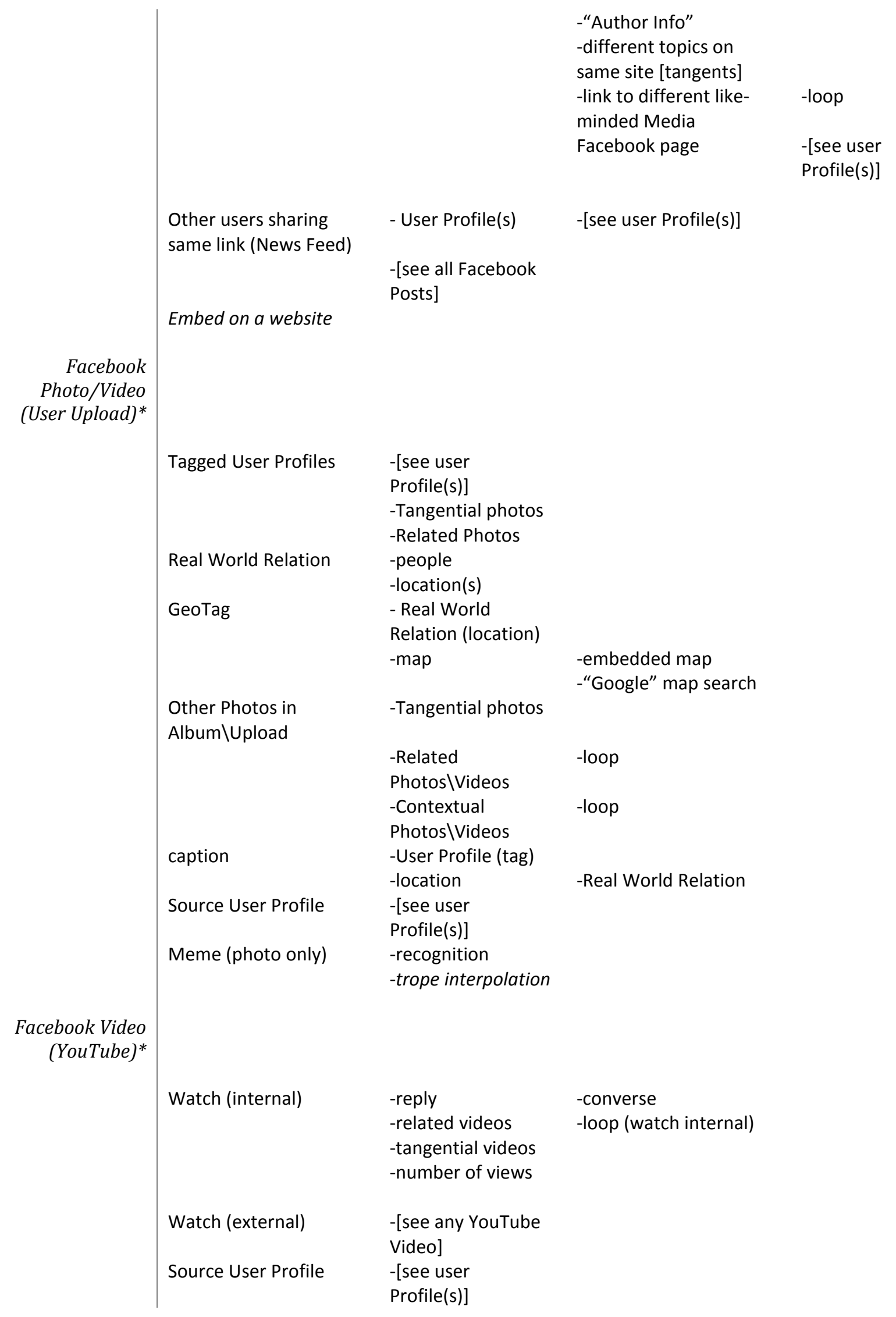




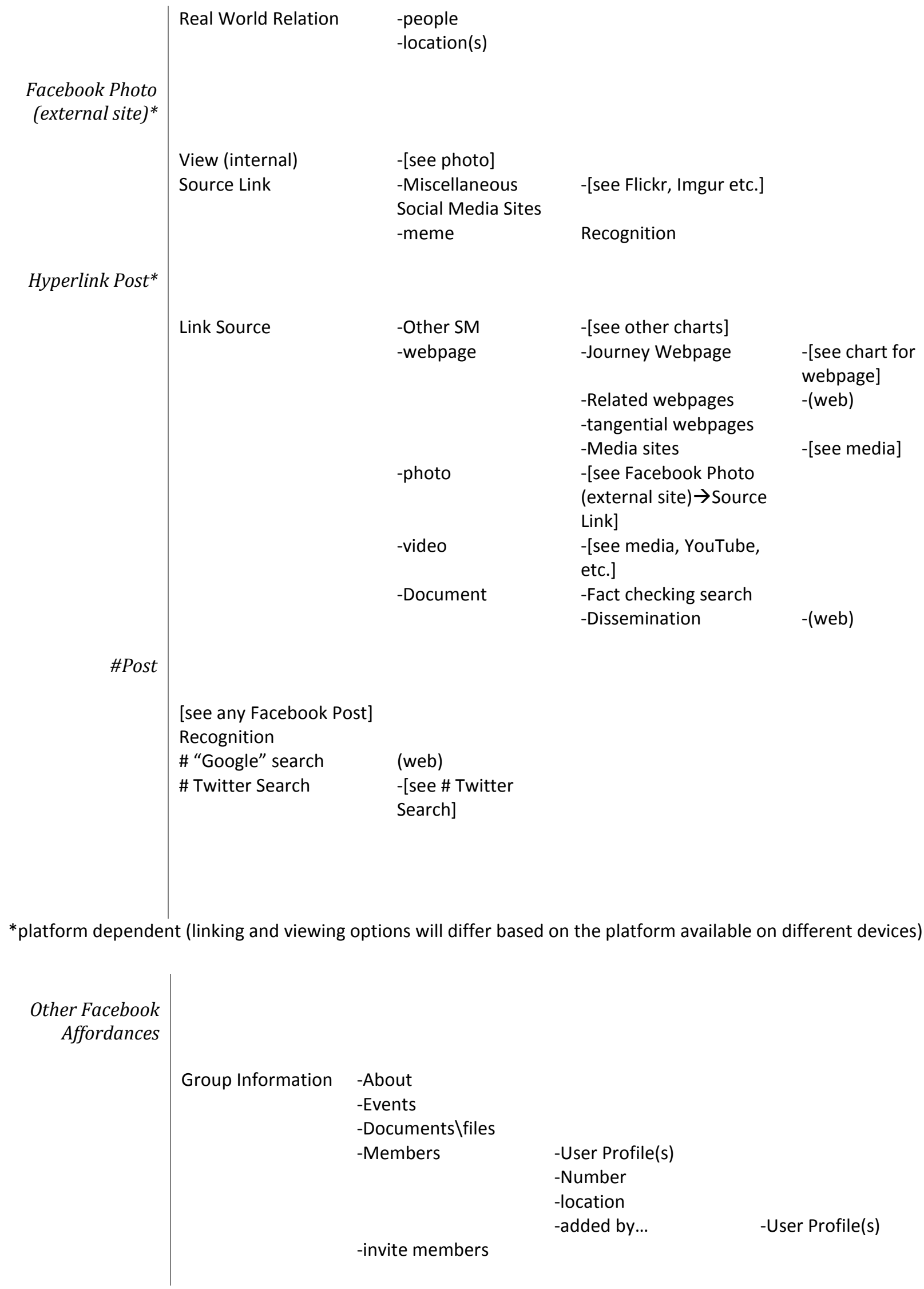




\begin{tabular}{|c|c|c|c|}
\hline \multirow[t]{2}{*}{$\begin{array}{l}\text { Personal } \\
\text { Information }\end{array}$} & -timeline \wall & $\begin{array}{l}\text {-[see all Facebook } \\
\text { Posts] }\end{array}$ & \multirow[b]{2}{*}{-location } \\
\hline & -About & $\begin{array}{l}\text {-contextual } \\
\text { information }\end{array}$ & \\
\hline & & & $\begin{array}{l}\text {-work \education } \\
\text { history } \\
\text {-self designed } \\
\text { "profile" }\end{array}$ \\
\hline & -photos & $\begin{array}{l}\text {-tangential } \\
\text { information } \\
\text {-profile photo }\end{array}$ & \\
\hline & & -personal albums & -related \\
\hline & & $\begin{array}{l}\text {-photos added by } \\
\text { others (tagged) }\end{array}$ & $\begin{array}{l}\text {-tangential } \\
\text {-related }\end{array}$ \\
\hline & & & -tangential \\
\hline & -Friends & $\begin{array}{l}\text {-Mutual Friends } \\
\text {-others (dependent on } \\
\text { privacy settings) }\end{array}$ & \\
\hline & -Events & $\begin{array}{l}\text {-created } \\
\text {-attending }\end{array}$ & $\begin{array}{l}\text {-related } \\
\text {-tangential } \\
\text {-related } \\
\text {-tangential }\end{array}$ \\
\hline & -Groups & -[see group profile] & \\
\hline & $\begin{array}{l}\text {-Tangential } \\
\text { information }\end{array}$ & & \\
\hline Community & -\#of “likes" & & \\
\hline & $\begin{array}{l}\text {-\#of users "talking } \\
\text { about this" } \\
\text {-photos }\end{array}$ & & \\
\hline
\end{tabular}




\section{Appendix "C": Figures}

\section{C.1}

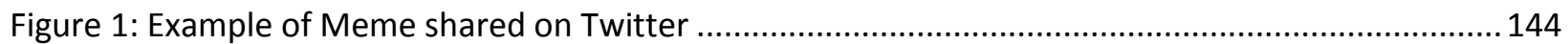

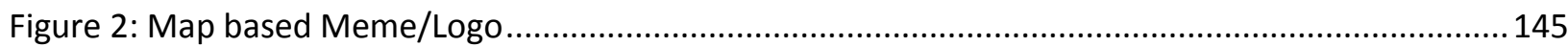

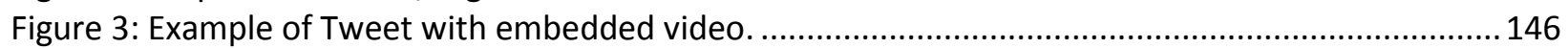

Figure 4: An example of a tweet linking to a news article................................................................. 147

Figure 5: An example of a Tweet linking to a Flickr account. .............................................................. 148

Figure 6: An example of a Tweet with an embedded photograph.................................................... 149

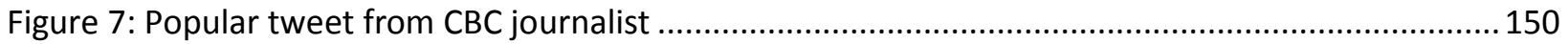

Figure 8: Showing an image/meme not directly related to the JON. ............................................... 151

Figure 9: Image of Walkers during trek posted on Facebook Feb. 24th.............................................. 152

Figure 10: Example of a shared Facebook post with dialogue in comments. ....................................... 155

Figure 11: An example of Alternative Media link(s) on a Facebook post. ............................................. 156

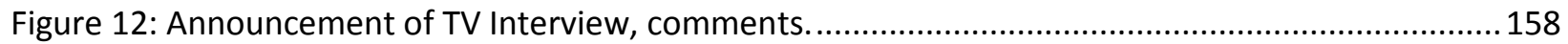

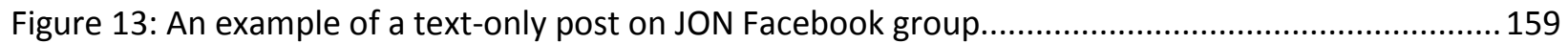

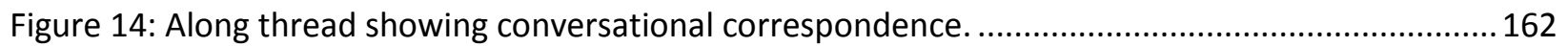

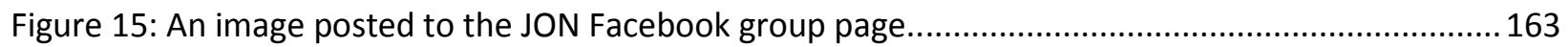

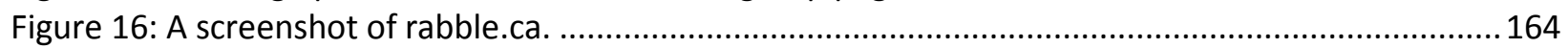

Figure 17: Comments section from Huffington Post article. .......................................................... 166

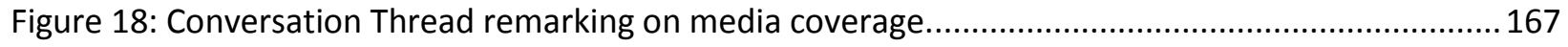

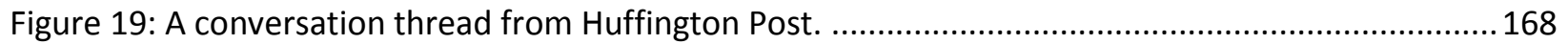

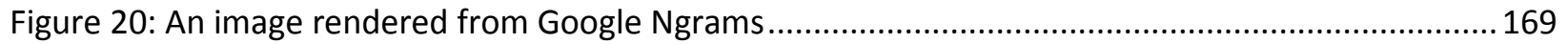

Figure 21: Map showing connectivity profiles of Aboriginal Communities in Quebec............................. 170

Figure 22: Comments section from an Ottawa Citizen News Article................................................. 171

Figure 23: An example of a text-only comments section. .............................................................. 174

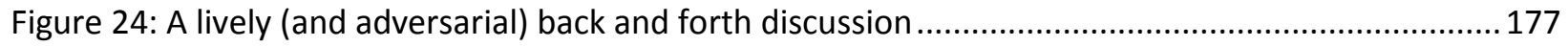

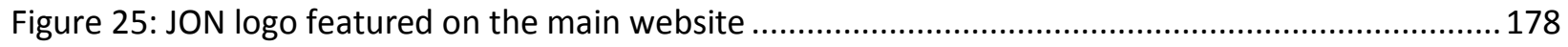

Figure 26: A tablet-drawn image comparing the JON to the Lord of the Rings Trilogy ........................... 179

Figure 27: Image of Google Earth map with points demarcating the JON trajectory. ............................ 180

\section{C.2}

Figure 28: Table showing top 36 \#Nishiyuu tweets by volume. ......................................................... 185

\section{C.3}

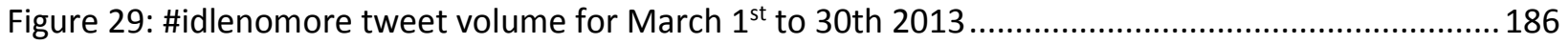

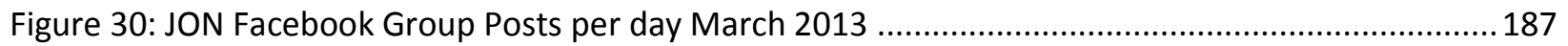

Figure 31: "Tweet volume of \#idlenomore hashtag adapted from Topsy. (Topsy, 2013)" ..................... 187

Figure 32: Volumetric Timeline for JON Facebook group page ......................................................... 187

Figure 33: Data showing \#idlenomore volumes on arrival of Nishiyuu walkers (March $25^{\text {th }} 2013$ )......... 188

Figure 34: Data showing idlenomore traffic for the Week of 3-9 March 2013 ....................................188

Figure 35: Data showing idlenomore traffic for the Week of 10-17 March 2013 .................................188

Figure 36: Data showing idlenomore traffic for the Week of 24-30 March 2013 ..................................189

Figure 37: Data showing idlenomore traffic for the Week of 24-30 March 2013 ................................... 189 


\section{C.1 Screenshots}

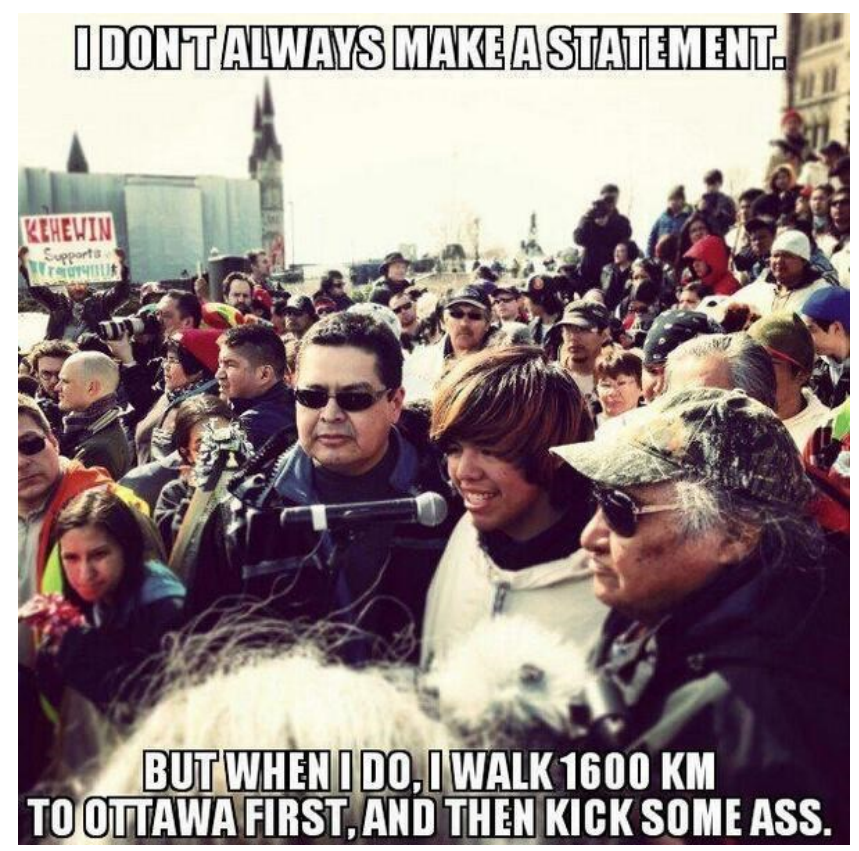

Figure 1: Example of Meme shared on Twitter ${ }^{399}$

\footnotetext{
${ }^{399}$ Meme Google image search “\#nishiyuu”
} 


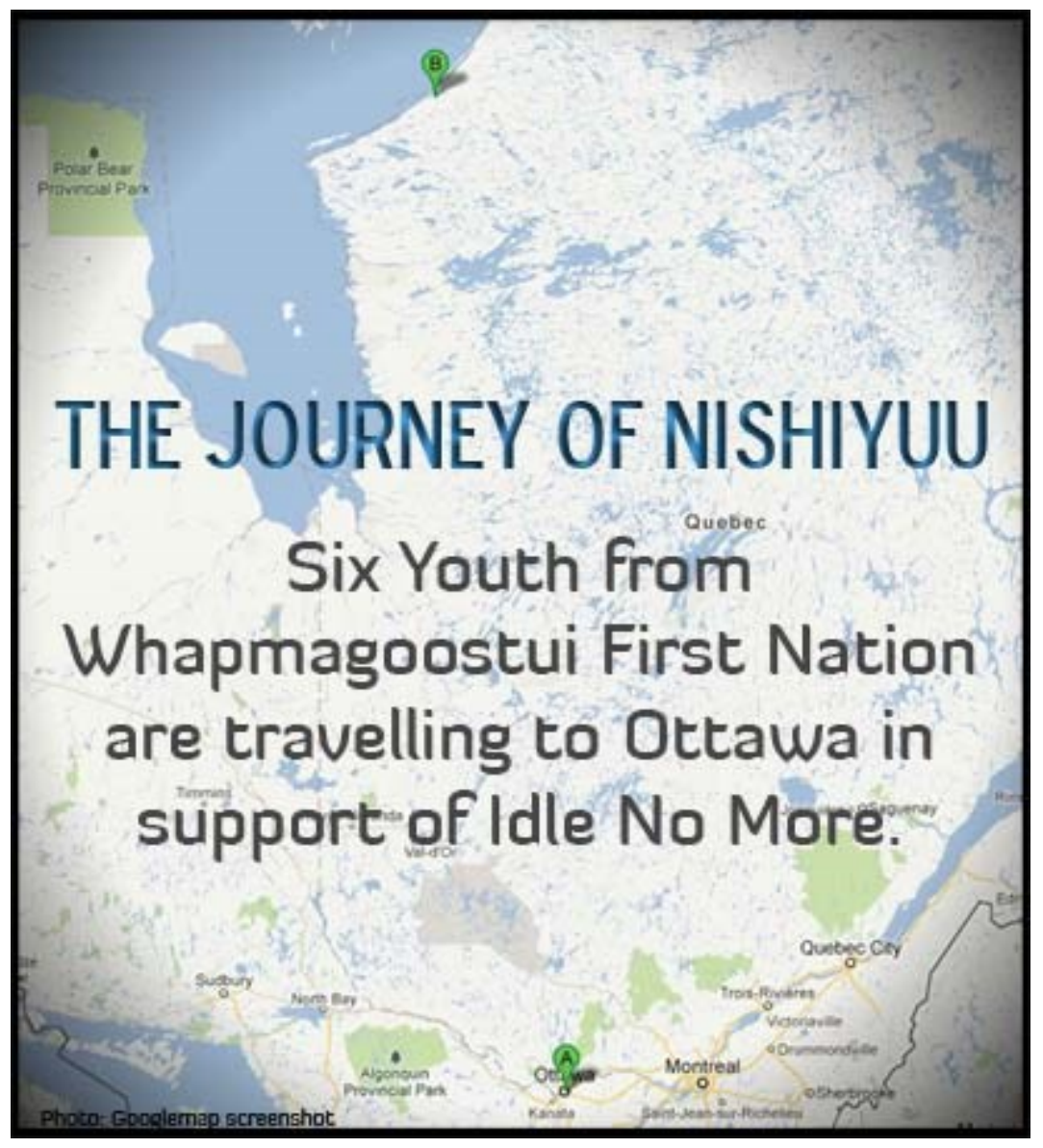

Figure 2: Map based Meme/Logo posted on the JON aggregator on Ahki.co400. This image was tweeted 36 times, Liked on Facebook 245 times from the Ahki page.

${ }^{400}$ Ahki (Last Modified: March 28 ${ }^{\text {th }}$ 2014) 


\section{Fateh Born}

t2 +2 Follow

@FatehBorn

\section{Beautiful summary of \#Nishiyuu walkers $1600 \mathrm{~km}$ journey mostly on} snowshoes.\#idlenomore youtu.be /jqqNVgfOQHO
4 Reply 27 Retweet « Favorite ... More
YouTube

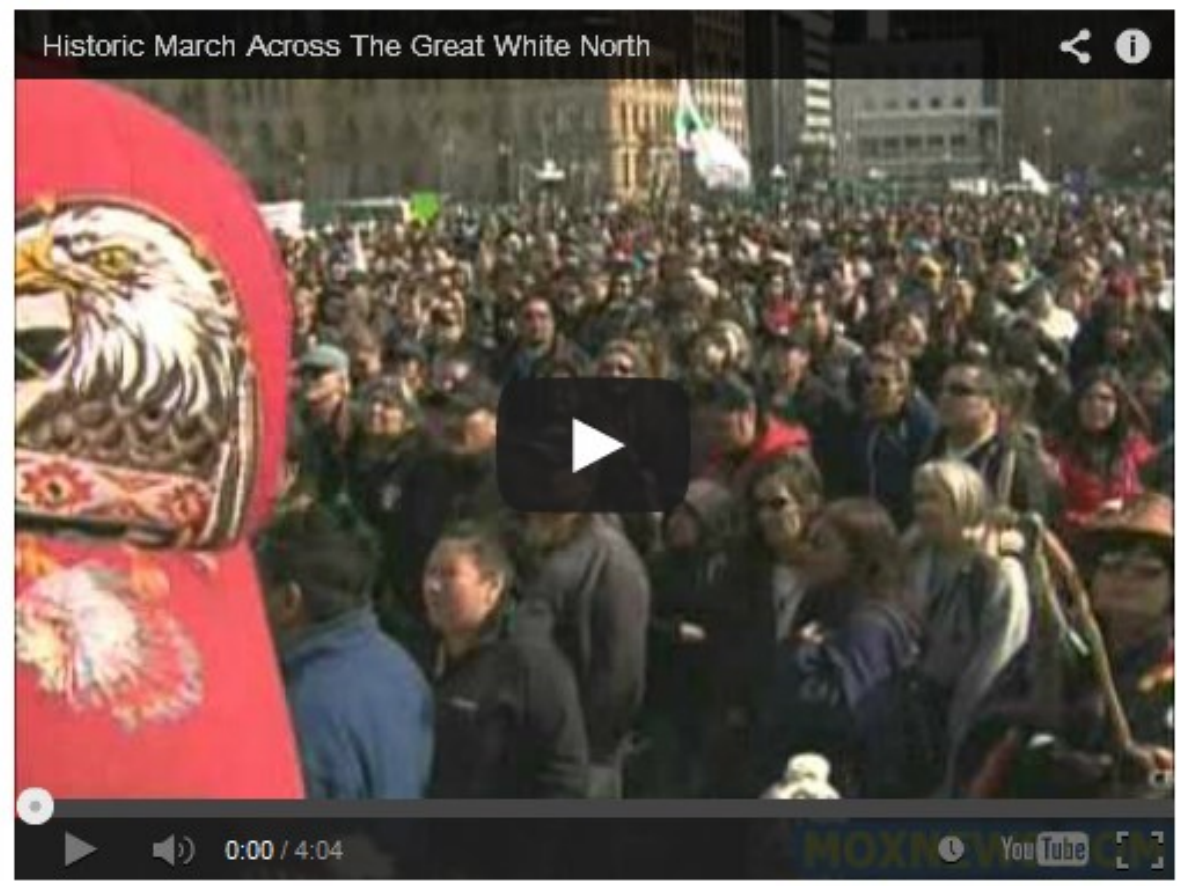

Historic March Across The Great White North

March 25, 2013 CBC News http://MOXNews.com

View on web

RETWEET

1

10:29 AM - 26 Mar 2013

Flag media

Figure 3: Example of Tweet with embedded video ${ }^{401}$. On a mobile device the video would be viewed externally through the YouTube app.

401 @FatehBorn (March 26 ${ }^{\text {th }} 2013$ ) 


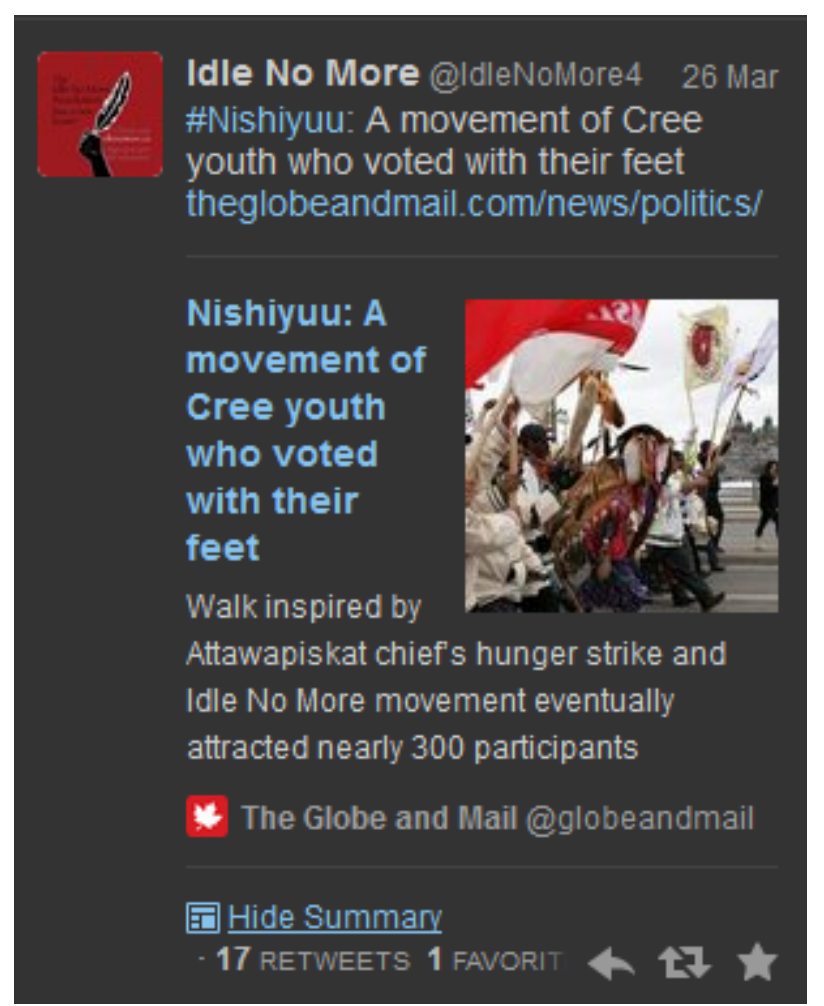

Figure 4: An example of a tweet linking to a news article ${ }^{402}$. This tweet includes a snippet of the article and an image. On a mobile device the summary would need to be expanded so would not immediately been shown.

$402 @$ Idlenomore4 (March 26 $\left.{ }^{\text {th }} 2013\right)$ 


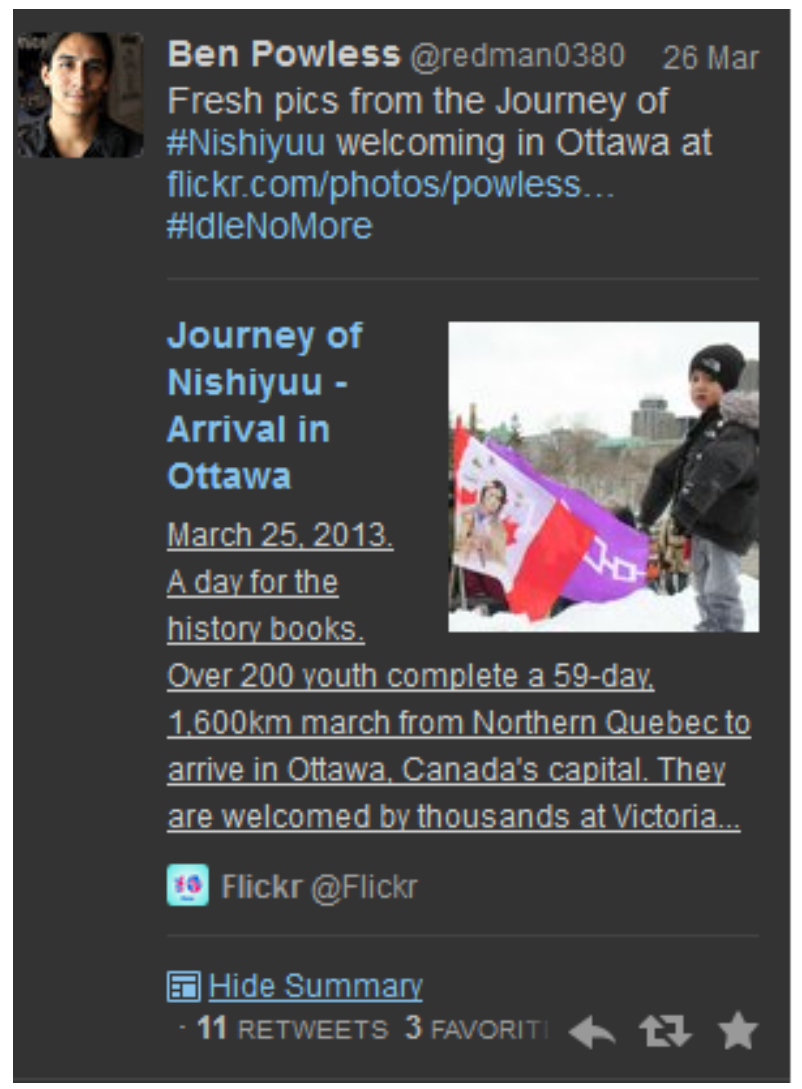

Figure 5: An example of a Tweet linking to a Flickr account ${ }^{403}$. This tweet also shows the use of \#Nishiyuu and \#idlenomore used in tandem.

${ }^{403}$ Powless, Ben (March 26, 2013) 
So proud of my cousin @Shawin29 who completed her journey today!! \#Nishiyuu \#IdleNoMore pic.twitter.com/fzmtx6iLKI

4 Reply 27 Retweet $\star$ Favorite ... More

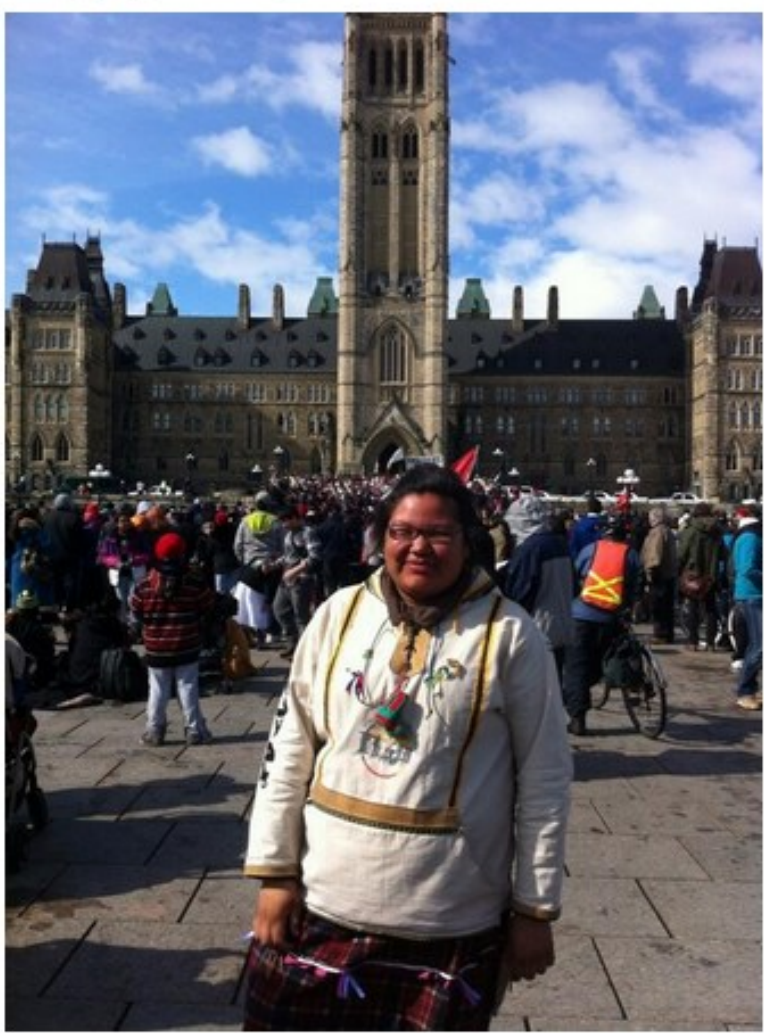

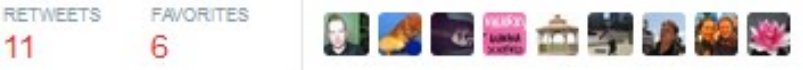

8:18 PM - 25 Mar 2013

\section{Reply to @eli_d_m}

Dale Odberg (1)dodberg-25 Mar 2013

@eli_d_m @Shawin29 Wonderful. Way to be! All the best

Details

4 Reply 2 ? Retweet $\star$ Favorite

... More

404 @eli_d_m (March 25 $5^{\text {th }}$ 2013) 
The \#Nishiyuu walkers are a true inspiration. And deserve more attention than visiting pandas. - my opening essay tmrw morn. $\# \mathrm{Q}$

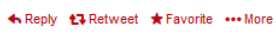

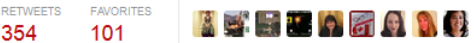

3.1. 101

Christi Belcourt @christibelcourt Mar 25

@jianghomeshi agree! I will be listening

Detalis $\quad$ Reply 27 Retweet $\star$ Favorite $\cdots$ More

RETWEe

1

3:54 PM - 25 Mar 2013 - Details

Reclaim Democracy eColettestweeter - Mar 25

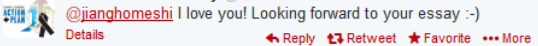

Kelly Patrick @kellypatrickxox - Mar 25

@Colettestweeter ejianghomeshi hold on to that love. CBC must hire aboriginal cultural mouthpieces if that's what CBC does. 1 or 2 would do. ↔ Reply 27 Retweet $\star$ Favorite .... More

8:49 PM - 25 Mar 2013 - Details

Reclaim Democracy @ Colettestweeter-Mar 25

5iii1. @kellypatrickxox@jianghomeshi Excellent ideal

Details $\$$ Reply tz Retweet $\star$ Favorite ... More

8:51 PM - 25 Mar 2013. Details

Kelly Patrick @kellypatrickxox - Mar 25

@jlanghomeshi @colettestweeter oh relax.

Details $\quad$ Reply 27 Retweet $\star$ Favorite .... More

8:58 PM - 25 Mar 2013 - Details

Reclaim Democracy@Colettestweeter - Mar 25

SFitil 18 @jianghomeshi @kellypatrickxox Yes! and you and \#Q are (\#HARPER) we are under

Details

9:04 PM - 25 Mar 2013 - Details

Kelly Patrick @kellypatricloxox - Mar 25

@jlanghomeshi who decides who fills in for you when you go on

Details $\quad$ \& Reply 2 t7 Retweet $\star$ Favorte $\cdots$ More

9:05 PM - 25 Mar 2013 - Details

Kelly Patrick @kellypatriclocox - Mar 25

What am I supposed to see? That and "now listen" is most
Whan

patronizing language.

9:09 PM - 25 Mar 2013 - Details

@jianghomeshi and no one elected me, I chose to do so on my

Details

9:10 PM - 25 Mar 2013 - Detals

jian ghomeshi @janghomeshi-Mar 25

@kellypatrickxox sorry. îm not interested in twitter fights with people swearing at me. we can be more productive than this. good

$\pitchfork$ Reply 27 Retweet $\star$ Favorite... More

2 \& 6

9:14 PM - 25 Mar 2013 - Details

Kelly Patrick @kellypatriclocox-Mar 25

- janghomeshil agree. I just spent two days in Thunder Bay and the racism is less than palatable. Please keep FN youth on radar. Peace

Detals

2:16 PM- 25 Mar 2013 - Details.

Figure 7: Popular tweet from CBC journalist Jian Ghomeshi stimulating a series of replies, 354 retweets, and 101 Favourites ${ }^{405}$.

$405 @$ jianghomeshi (March 25 $\left.5^{\text {th }} 2013\right)$ 
Mina Elizabeth Bearskin shared Lessons Learned In Life's photo.

\section{Life is made up of special moments} which make it worth living. There are many cherished moments that are missed due to the stress and fast pace of daily living. We must slow down and remember how precious it is to be alive and to love.

\section{Lessons Learned In Life}

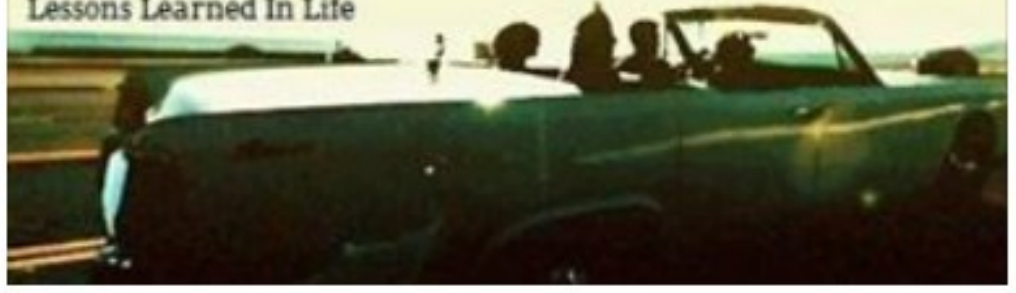

Like ' Comment - Share · March 11, 2013 at 1:19am

3 people like this.

Figure 8: Showing an image/meme not directly related to the JON ${ }^{406}$. This shows the page being used as a site for an online community beyond the protest itself.

406 Bearskin, Mina Elizabeth (March $11^{\text {th }}$ 2013) 


\section{Jimmy Tim Whiskeychan}

February 24, 2013

On their way to km 98- Feb 24,2013 in Waskaganish

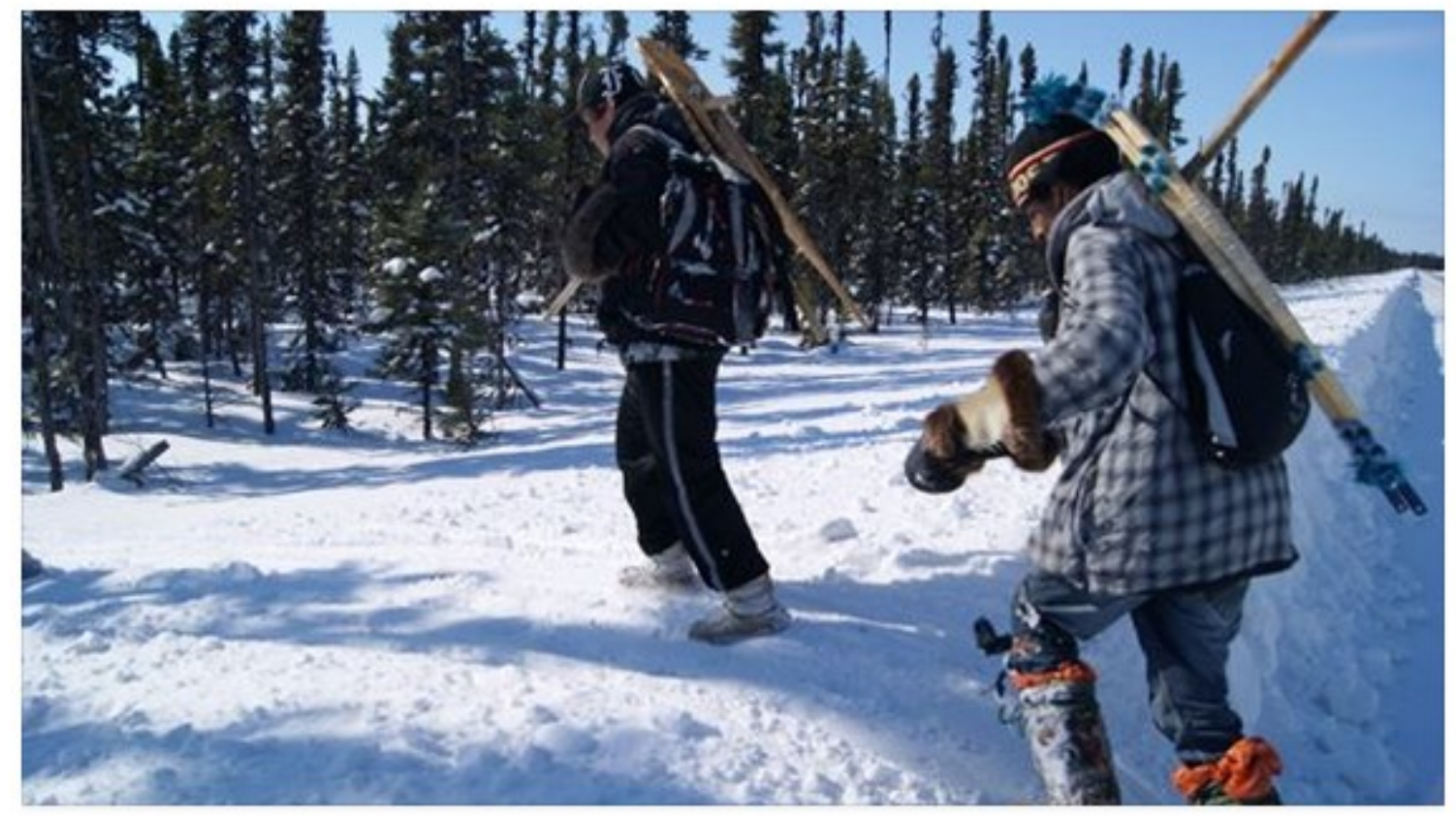

Like · Comment · Share

\section{1 people like this.}

Write a comment...

Figure 9: Image of Walkers during trek posted on Facebook Feb. 24th ${ }^{407}$

${ }^{407}$ Whiskeychan, Jimmy Tim (February 24, 2013) 


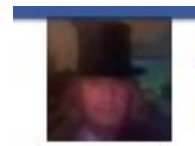

\section{Andy Barham > The Journey of Nishiyuu}

May $14,2013 \cdot \theta$

Sadly, the mainstream media paid even less attention to this long trek than they did to yours. However, I would be willing to bet money that if a group of White, middle class pensioners walked $50 \mathrm{~km}$ (or probably not even that much) to protest a small tax on their pensions, the bastards would have been out in full force to cover the event.

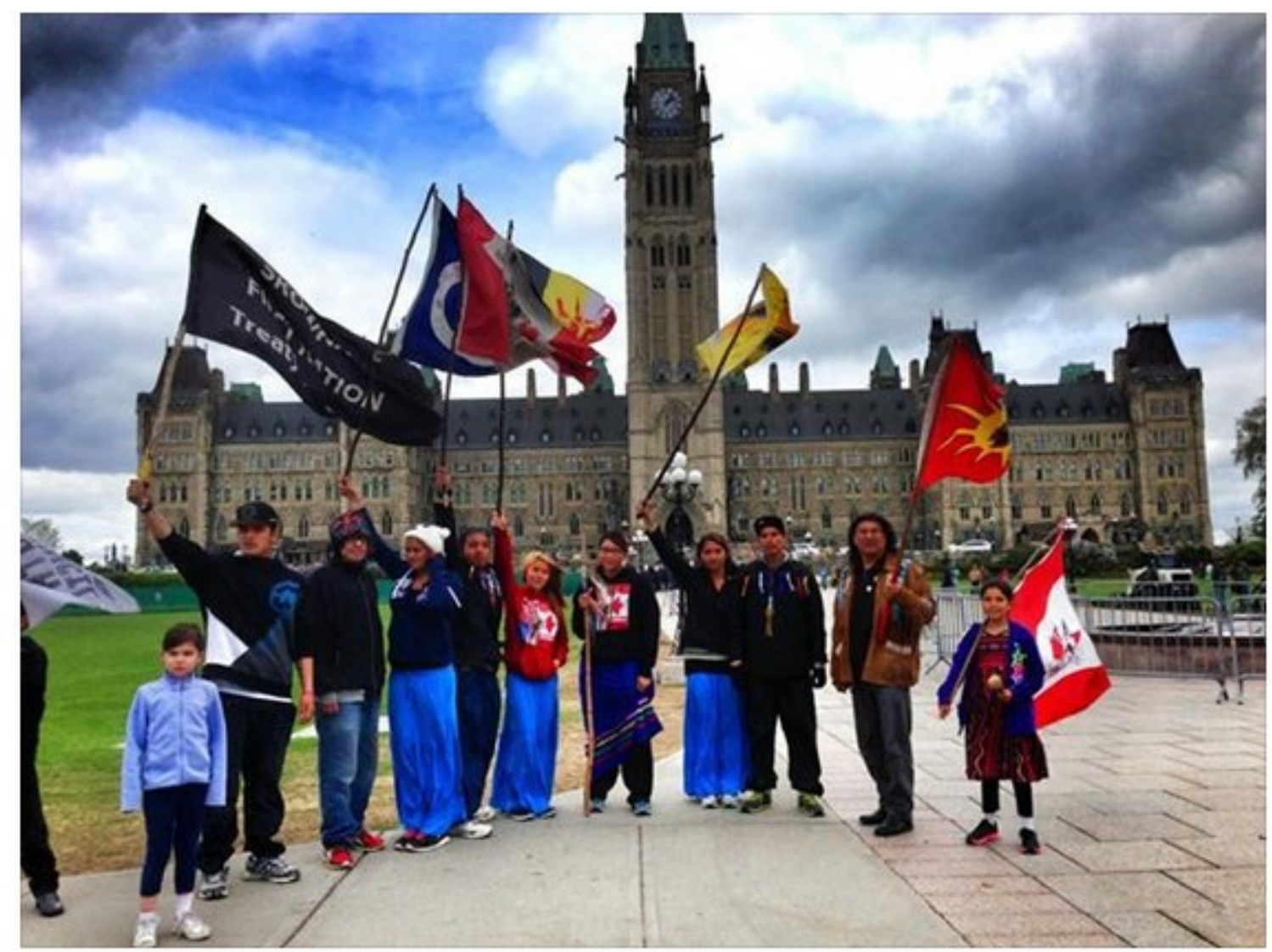

\#IdleNoMore .. Y4L Warrior walkers arrive in Ottawa today after 45 day journey from Winnipeg, Manitoba, Canada! .. May 13th 2013

CONGRATULATIONS : They walked to protect the rivers, lakes and streams from HARPER and C-38 \& C-45!

It would have been nice if mainstream media and Canadians supported these Warriors more than they have been.

Photo via Youth 4 lakes

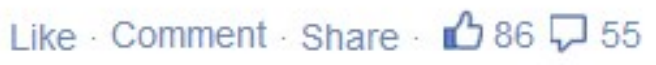


38 people like this.

Andy Barham Occupiers from Victoria and Vancouver trekked all the way

across Canada to Ottawa shortly after all the Occupiers got the boot from cities and towns across this country and around the world, but there was no coverage at all in the media. They don't care about it, it's that simple.

May 14, 2013 at 11:05am - Like - 凸3

Diane Corbin Media and politicians may have disappointed. What else is

new? But at the level of pure consciousness the energy has been raised. This is important. Much patience is required. And I bet each of the walkers has learned so much. This is also important. I think we have to focus far ahead. We each have to continue to stand up and do something. In the words of Gandhi, "First they ignore you, then they laugh at you, then they fight you, then you win." Awesome job Y4L!! On a practical level, maybe PR needs to be more organized? May 14, 2013 at 11:40am - Like $\cdot$ B

Mary Slipperjack That is an awsome accomplishment very proud if all of you's May 14. 2013 at 12:09pm - Like - B Z

Se Deborah Stark Diane wrote

"......Media and politicians may have disappointed. What else is new? But at the level of pure consciousness the energy has been raised. This is important....."

Yes, this is VERY important. This is in fact the HEART of the matter. "Media and politicians" will almost ALWAYS disappoint as long as we continue to depend on them to acknowledge (fat chance) what is real and true. We can SEE for ourselves what is happening to the life-support systems upon which we all depend for our survival and we CAN, with patience and unified effort, DO something to slow down and ultimately stop most of the damage. The young people are READY to work for a better world. Are we, their parents and grandparents, READY to support them? I know I am!!! Let's GO!!!

May 14, 2013 at 12:24pm - Like - B 11

(3. Tammy Racicot ::) icare!!!! They survived and accomplished, no one in this or the next generation would even consider trying to do or even succeed! My children wouldnt be able to do it, and i feel that what this walk proved was that traditions, values and beliefs when maintained conquer all odds and maintain survival and wisdom from that experience. I feel your movement is the catalyst to save whats left or the environment and a reminder of what everyone lost in lieu of technology and a so called better future. No one ever defined for whom, so I think, its definitely time to take notice. Keep fighting the good fight Warriors, I stand beside such a noble cause!! $O$

May 14, 2013 at $1: 14 \mathrm{pm} \cdot$ Like $\cdot$ B 8

Q Robert Savoie Not proud of your comment Andy Barham and very surprised that some middle class white pensioners like it

Mav 14. 2013 at 4:26 pm - Like - B 7

Deborah Stark Good point, Robert.

May 14, 2013 at 5:22pm · Like - @2

Bob Mullins Andy Barnham, i am one of those that you mentioned and i find your post frigging insulting (:) (:)

May 14, 2013 at 5:42pm - Like B 4

7. Brenda Ross I have to admit I was saddened by this comment. I am white. Not a choice, I was born that way I can't even say I'm proud to be white. I' $m$ not even proud to be human most days. I do; however, love my Mother (earth) and am rooted in many spiritual value... See More

May 14, 2013 at 5:52pm - Like - B 17

1. Bob Mullins You can remove this post or it will be by FB. It has been reported May 14. 2013 at 6:09pm · Like : B2

Tom Harbold I'm not sure which thread to post this in, but I'm sure our esteemed moderators will find a good home for it if this is not appropriate! Someone posting on the Sacred Journey for Future Generations page raised the idea of turning away from Parliament Hill, walking to a place of beauty, and declaring a Capitol of Turtle Island. Not sure how others feel, but I actually kind of like that idea. (:) A Capitol for the entire North American continent, a Nationof-many-Nations to which those who love Mother Earth and honor the Ancestors and the Creator could "swear allegiance" - symbolically, at least, and who knows, maybe someday actually - and which hopefully would bring together Indigenous Peoples and their Allies to care for and protect this good land and all its inhabitants. Maybe I'm just too idealistic, but I like it. (:)

May 14. 2013 at 8:16pm · Like : B 7

Brenda Ross $\bigcirc$ to $\bigcirc$

May 14, 2013 at 8:17pm · Like $\cdot 凸 3$

Deborah Stark Tom and Brenda... wow. Each of you has said, word for word, exactly what I could have said myself. I could not possibly be more in agreemen with either of you. And I think the idea of an alternate Capitol for the entire North American continent is electrifying... and for exactly the reasons you state, Tom. Even if it is for the time being only symbolic I think it's eminently worth aspiring to May 14, 2013 at 8:45pm - Like : 5

David Smith I remind folks that fb does not provide a button to "dislike" posts The rationale is that you're simply acknowledging that you have read the post... Don't like it but that's what I'm told. May 14, 2013 at 10:54pm · Like $B$ 
Figure 10: Example of a shared Facebook post with dialogue in comments ${ }^{408}$. The post is shared by 'Andy', but was originally posted by 'Youth 4 Lakes'. The original caption for the image is at the bottom, and the comment by the poster above the image.

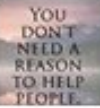

Darin Howard shared his photo.

March 31, 2013 · Kelowna

Since major media wouldn't cover it...

https://www.facebook.com/photo.php?fbid=10151579888911420\&

set=0.192688097431155\&type=1\&relevant_count=1\&ref=nf

We did...

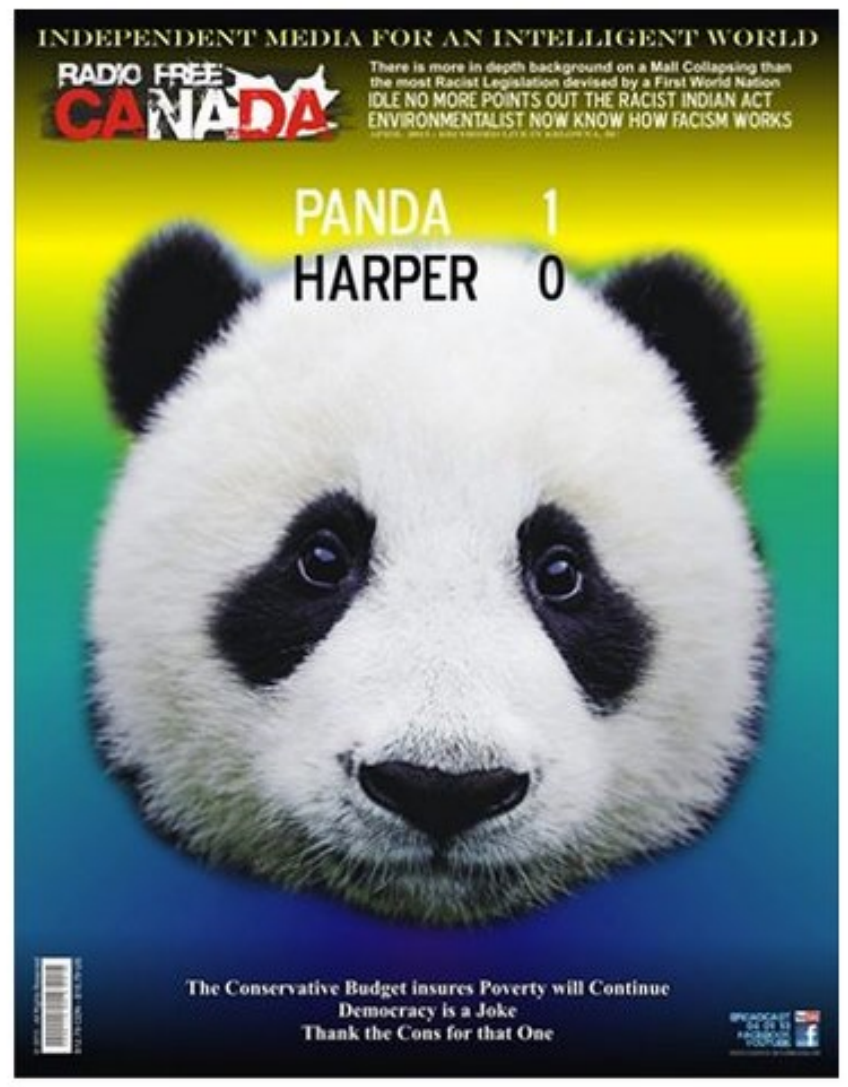

Stephen Harper has our attention:

Idle No More Update - Part I http://youtu.be/2rE9bymP-XU

The Panda-Budget - Part II http://youtu.be/2GLql5jKADs

because Major Media can't handle the truth

We have to thank www.abdulbutt.com for being on the ground and doing some awesome stuff... cheers!

Like · Comment - Share

408 Barham, Andy (May 14 ${ }^{\text {th }}$ 2013) 
Figure 11: An example of Alternative Media link(s) on a Facebook post ${ }^{409}$. Features a digitally rendered image/advertisement, with hyperlinks internal to Facebook, to an external site and to YouTube videos (not embedded).

${ }^{409}$ Howard, Darrin (March $31^{\text {st }} 2013$ ) 
David Kawapit

March 24, 2013 - Wakefield

CBC interview with David Kawapit live at 3:00 PM Eastern Time.

Like - Comment - Share

B 148 people like this.

Bob Mullins Thanks (:)

March 24, 2013 at 2:53pm - Like - B1

Basil George Wow! Thanks.

March 24, 2013 at 2:57pm - Like

Wh. Carla Iserhoff what channel in shaw direct........Iol

March 24, 2013 at 2:59pm - Like

Beverly Soulier Ouuwah!

March 24, 2013 at 2:59pm - Like

Kathleen Pepabano What channel

March 24, 2013 at 3:01pm - Like

Bob Mullins CBC is different for each satellite provider !

March 24, 2013 at 3:08pm - Like

Carla Iserhoff yuh....Iol, thats why i wrote shaw direct....:

March 24, 2013 at 3:08pm - Like

Martha Matthew Channel 20 in Chisasibi.

March 24, 2013 at 3:11pm - Like - 1

Kathleen Pepabano Watching it now channel 502 bell

March 24, 2013 at 3:11pm - Like - B2

Bob Mullins http://www.shawdirect.ca/.../channel_listing_c_10_12_e.pdf

March 24, 2013 at 3:13pm - Like - B1

Desiree Jamie Wabigoun Ottereyes You were AWESOME! (:)

March 24, 2013 at 3:19pm - Like - B1

Beverly Soulier You were Great!

March 24, 2013 at 3:20pm - Like

Bob Mullins WOW David so proud of you $\bigcirc \odot$

March 24, 2013 at 3:20pm - Like - 1

Bob Mullins 9

March 24, 2013 at 3:23pm - Like

Fred DelforgeMahikan tv direct internet no?

March 24, 2013 at 3:31pm - Like

8. Sarah Scott Is there a web link for it.. Just gt back from shopping in Ottawa

F. $>$ : $p$

March 24, 2013 at 3:37pm - Like - @ 2

Bob Mullins Sarah,it was live on CBC TV. Maybe it will be archived then there will be a link (:)

March 24, 2013 at 3:42pm - Like - 2

Bob Mullins@ @red, your profile does not say where you live so unable to get info! 
Stella M. Wapachee Awesome. You're an inspiration!!

March 24, 2013 at 3:44pm - Like - B 2

Janet Turnbull It's on right now in Ontario 4:14

March 24, 2013 at 4:15pm - Like - B1

Myna Lee Johnstone and the Ambassador from China which means FIPA

March 24, 2013 at 4:17pm - Like

Andrew Neeposh You did great buddy!!

E1 March 24, 2013 at 4:19pm - Like

Jean E. Ryan just caught the end of the interview....very, very good David $O$

March 24, 2013 at 4:25pm - Like

Janet Turnbull All you walkers are amazing, and you are heard by us all! 0

March 24, 2013 at 4:27pm - Like - B1

Craig Heber You did a wicked job ... Kudos for speaking for Chief Spence and asserting it is to Unite First Nations and all Canadians $O$

March 24, 2013 at 4:33pm - Like - $B 2$

Se

Deborah Stark I hope this will be parked on the CBC web site so we can all see it later. (:)

March 24, 2013 at 4:36pm - Like - 33

10

Jennie Wright And I missed it! I was baking buns for tomorrow. Janet

Turnbull, you have got to be kidding! Maybe if we all put on polar bear suits they'd pay attention to us.

March 24, 2013 at 4:37pm - Like

Jennie Wright Oops, I meant panda bear suits. It's panda bears that they're meeting, I believe.

March 24, 2013 at 4:39pm - Like - B1

Jude Johnston Missed it, send link pretty please if one surfaces...! (:)

March 24, 2013 at 4:51 pm - Like - B1

Bruce Gamble yep need a link if possible! :(:)

March 24, 2013 at 5:02pm - Like - B1

Jude Johnston Any summary anyone care to share? (thoughts, words), if it seems appropriate.

March 24, 2013 at 5:05pm - Like

Jude Johnston ps that were in the interview...

March 24, 2013 at 5:05pm - Like

Q.

Becky Catts Knutson I hope they repeat on the news later on today...missed it! March 24, 2013 at 5:30pm - Like - 1

Bob Mullins Becky, hope for 6.12 PM and keep your fingers crossed (:) ::) March 24, 2013 at 5:46pm - Like

a.7. Linda Lameboy I saw it, awesome interview David.

Ifip March 24, 2013 at 6:27pm - Like - B1

1.5 Jennie Wright Aargh, I missed it again!! :)

March 24, 2013 at 6:29pm - Like - B1

20 Patricia George If someone finds the link, please share.

March 24, 2013 at 6:50 pm - Like - B2

Violet Bearskin I watched, David was awesome !!!! It would be great if He would lead the walkers to the Parliament Hill!!!

March 24, 2013 at 11:07pm - Like - B1

Write a comment..

Figure 12: Announcement of TV Interview, comments ${ }^{410}$. Commenters discuss logistics of viewing and comment after having viewed the Television broadcast both online and on conventional television.

${ }^{410}$ Kawapit, David (March 24, 2013) 
First of all I would like to say, a lot of healing has been done from this journey, not only for the walkers, but, for all of us who followed them since day 1

I also have been following them since they started in our community of Whapmagoostui.

There was never a day I missed without crying. before they left on their journey, The original 7 came to see me at my house, I didn't even know they were gonna stop by before they left. I was really surprised when they came into my room, I will never forget that

Even though I'm wheelchair bound, I walked with them, in spirit. Three of my cousins told me before they left that, they were going to do this walk for me. I was really touched when they told me. I'm somehow related to the original 7 .

To the Original 7 and all the walkers from Whapmagoostui, I will see you when you get back in Whapmagoostui. I will be waiting

All My relations.

Like - Comment - Share

118 people like this

Norman Ottereyes I was thinking about you today my friend hope to see you take care March 25, 2013 at 6:57pm - Like - ß

6. Janet Turnbull Norman, your song brought me to tears - what an amazin talent you have 0 March 25, 2013 at 7:00pm - Like - B

Roxane Landry 7 young men are a true inspiration and to make their speeches in Cree brought tear's to my eye's. Mahsi cho, all my heart March 25, 2013 at 7:01pm - Like - 15

Shirley Sandy 0

March 25, 2013 at 7:27pm - Like - B

Carou Huptido Inspiring the people

March 25, 2013 at 7:28pm- Like - M

$\mathrm{N}$

(2) pictures on here of them visiting you

March 25, 2013 at 7:45pm - Like - B5

aXI Minnie Masty $C$

9. March 25,2013 at $8: 14 \mathrm{pm}$ - Like - B 1

X Jan Lou Wonderful message, Buckley George. I remember seeing the photos from when they visited you before leaving and it was easy to see how important you are to each other. think you were a big inspiration for them the journey. (:) March 25, 2013 at 8:19pm - Like - B

Marilyn Imerson why would mr harper put pandas before the bravery of the Nishiyuu walkers and welcome them, i live in uk but have been following the walk on $\mathrm{fb} i \mathrm{am}$ disgusted at such a blatant insult to the Indian nation March 25, 2013 at 8:19pm - Like - B 3

[4.] Jennifer Rodgers I remember the pictures too and thought of you today, Buckley \& wondered how you must be feeling. Thank-you for posting this message. I hoped you would write something.

March 25, 2013 at 9:09pm - Like - B 2

2. Maggie L Bugden We'll he (Harper) dubbed them my "national treasures" March 25, 2013 at 9:12pm - Like - B

2. Janet Turnbull @ Marilyn, it's his own stupidity... he lost a hell of a lot of

votes today.. but then he is known as the most unpopular prime minister an his words mean nothing March 25, 2013 at 10:33pm - Like - B3

Jude Johnston Buckley, you yourself are very inspiring. To see your appreciation of their visit and comments, well. You have heart. March 25, 2013 at 10:58pm - Like - B 2

72. Sandigrah Asin Buckley I too was so moved by the early photos and comments from your mum I think. We all provide each other with opportunities to be great. That's what interconnectedness is.. Love your post on here and please keep sharing with us, you have many new friends waiting in the guise of strangers! March 25, 2013 at $11: 52 \mathrm{pm}$ - Like - 4

Write a comment.

Figure 13: an example of a text-only post on JON Facebook group ${ }^{411}$

${ }^{411}$ George, Buckley (March 25 2013) 
Bob Mullins asked a question

March 14, 2013

Please use this page for general discussions

\begin{tabular}{|l|l|}
\hline $\begin{array}{l}\text { To awaken the nation through unity } \\
\text { and truth; to change the course of } \\
\text { humanity. }\end{array}$ \\
\begin{tabular}{|l|l|}
\hline This is a great idea Bob,,thank you & +245 \\
\hline $\begin{array}{l}\text { Hope it will preasure the gouverments } \\
\text { over our demands! :D }\end{array}$ & +50 \\
\hline
\end{tabular} \\
\hline
\end{tabular}

Like - Comment - Share

167 people like this.

View previous comments

Bob Mullins A bit of rest with a good supper will do wonders.

TTYL my friends (:)

March 25, 2013 at 6:18pm - Like

Bob Mullins A good day to my friends and extended family.

Was thinking that i would bring the discussion page up due to the popularity.

This will be post \#1213 (:)

March 26, 2013 at 11:58am - Like - 12

Bob Mullins For your info, there has been 666 posts in the last 24 hours !

March 26, 2013 at 12:01pm - Like - 33

Ada Hearn this could be a record of sorts!!

March 26, 2013 at 12:02pm - Like - 1

Bob Mullins Ada i would not even try to guess at the number of posts and $x$ yes it could be a record (:)

March 26, 2013 at 12:27pm - Like - 132

Bob Mullins Good day Ginette, it was nice to see your post on the other

page, have a great afternoon (:)

March 26, 2013 at 2:43pm - Like

Bob Mullins Good afternoon Bev (:)

March 26, 2013 at 3:17pm - Like

Bob Mullins Good afternoon Freda (:)

March 26, 2013 at 3:32pm - Like

Bob Mullins Good afternoon Jane (:)

March 26, 2013 at 3:47 pm - Like - 1

Bob Mullins Ok let's hear about your trip and your fun (:)

March 26, 2013 at 4:27pm - Like

Bob Mullins Good afternoon Kelly, what do you have to say (:) 
Marlene Westfall lol

March 23, 2013 at 10:28pm - Like - 1

Bob Mullins LMBO Marlene :)

March 23, 2013 at 10:30pm - Like - 32

Bob Mullins Kelly is having fun reading posts :)

March 23, 2013 at 10:33pm - Like - B 2

Ex] Kelly Masterson Oh yah... hehehe

ax. March 23, 2013 at $10: 35 \mathrm{pm}$ - Like - B 2

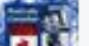

Kelly Masterson Here's a tune to celebrate one of our last nights together...

a19.

in our little FB house here... (-:) http://www.youtube.com

Iwatch?v=0 beCH7ToT $1 \mathrm{k}$

March 23, 2013 at 10:38pm - Like - @2

ति

Marlene Westfall no really it's nasty...that damn soft dog food - hey Bo

where are you located anyway?

March 23, 2013 at 10:39pm - Like

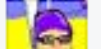

Marlene Westfall dang...i just butted my cigarette into my eyeglass case eyes are still adjusting to the lights on hehe

March 23, 2013 at 10:40 pm - Like - B 1

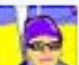

Marlene Westfall not to worry Kelly - I think the Nishyuu have united us here so we will probably keep our extended family $\odot$

March 23, 2013 at 10:42pm - Like - 132

Bob Mullins Marlene i live in Sherbrooke QC in the Fleurimont sector (:)

March 23, 2013 at 10:46 pm - Like - B 1

Marlene Westfall (:) parlez vous francais?

March 23, 2013 at 10:46 pm - Like - B 1

Marlene Westfall you know....I see all the same pic on the comments and $\mathrm{i}$

think i'm talking to myself! ... Our House...is a very very very fine house

March 23, 2013 at 10:50pm - Like - B 3

(A) Beverly Soulier Yay! Team

March 23, 2013 at 10:53pm - Like - B 2

Bob Mullins Google: Fleurimont, QC

March 23, 2013 at 10:54pm - Like

Bob Mullins House with a van in the yard and my garage to the right :)

March 23, 2013 at 10:55pm - Like - 11

Marlene Westfall cool - nice house can i write you and send you some seeds from my garden?

March 23, 2013 at 10:57pm - Like - 11

Marlene Westfall those who haven't friended me - please do so we can

keep in touch svp - that goes for you too stanley george jr

March 23, 2013 at 10:58 pm - Like - 32

Bob Mullins Done Marlene (:)

March 23, 2013 at 11:12pm - Like - B 1

Bob Mullins $\square$

March 23, 2013 at 11:29pm - Like - 131

Bob Mullins Those who want to be friends, feel free (:) 
$[\ldots]$

Gloria Harman Yikes... it's afternoon! not morning! l've been up for so long,

my day is nearly over! lol!

April 22, 2013 at 12:22pm - Like - 1

Bob Mullins Good morning Gloria, yep it is afternoon Iol. Have a Happy Earth

Day (:)

April 22, 2013 at $12: 36 \mathrm{pm}$ - Like - 1

Bob Mullins Trees are really budding today, better late than never lol

April 22, 2013 at 4:54pm - Like

Bob Mullins BRB supper !

April 22, 2013 at 5:43pm - Like

Bob Mullins Had a light supper but was delicious :)

April 22, 2013 at 8:19pm - Like

Bob Mullins Good night Gloria (:)

April 22, 2013 at 8:20pm - Like

Bob Mullins Anita, i try to keep troublemakers away as i have since Jan 16th. Most of them are trolls and activist. Had 3 removed in the past week. Thank you for not being offended by some of my responses! April 22, 2013 at 8:32pm - Like - 12

Anita Parker It is nice to know that there are people who dedicate themselves to that nasty task. Thank you (:)

April 22, 2013 at 8:39pm - Like - 4

Gloria Harman Anita, he growls occasionally, but he has kept this running very smoothly... seems there are a number of folk out there who try to keep the pot boiling.

April 22, 2013 at 9:03pm - Like - 33

Gloria Harman Sorry, Bo! You know you do! lol! It works! Grrrr!

April 22, 2013 at 9:03pm - Like - 131

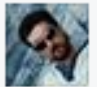

Robert Savoie Someone has to say: Sorry Pauline (:) I'm not a moderator, I'm still trying to figure out what happened. However, we must all question ourselves: was there something I could have said to defused the situation? April 22, 2013 at 9:05pm - Like - $\mathbf{B} 1$

Bob Mullins That was about the sense of the message i sent her !

April 22, 2013 at 9:32pm - Like

Nancy Howden Cowell Gloria Harman I looked that book up that you mentioned on the other thread, it looks like it would be a great read..I am going to see if the library has it or if they will order it...thanks for the review:) April 22, 2013 at 9:35pm - Like - 1

Figure 14: Along thread showing conversational correspondence ${ }^{412}$. This is the most commented upon thread on the JON Facebook page, with many comments coming from the same participants.

\footnotetext{
${ }^{412}$ Mullins, Bob https://www.facebook.com/groups/nishiyuu/permalink/384976824943032/; Bob Mullins provided his full address, but I felt it would be best if I redacted it for his privacy
} 


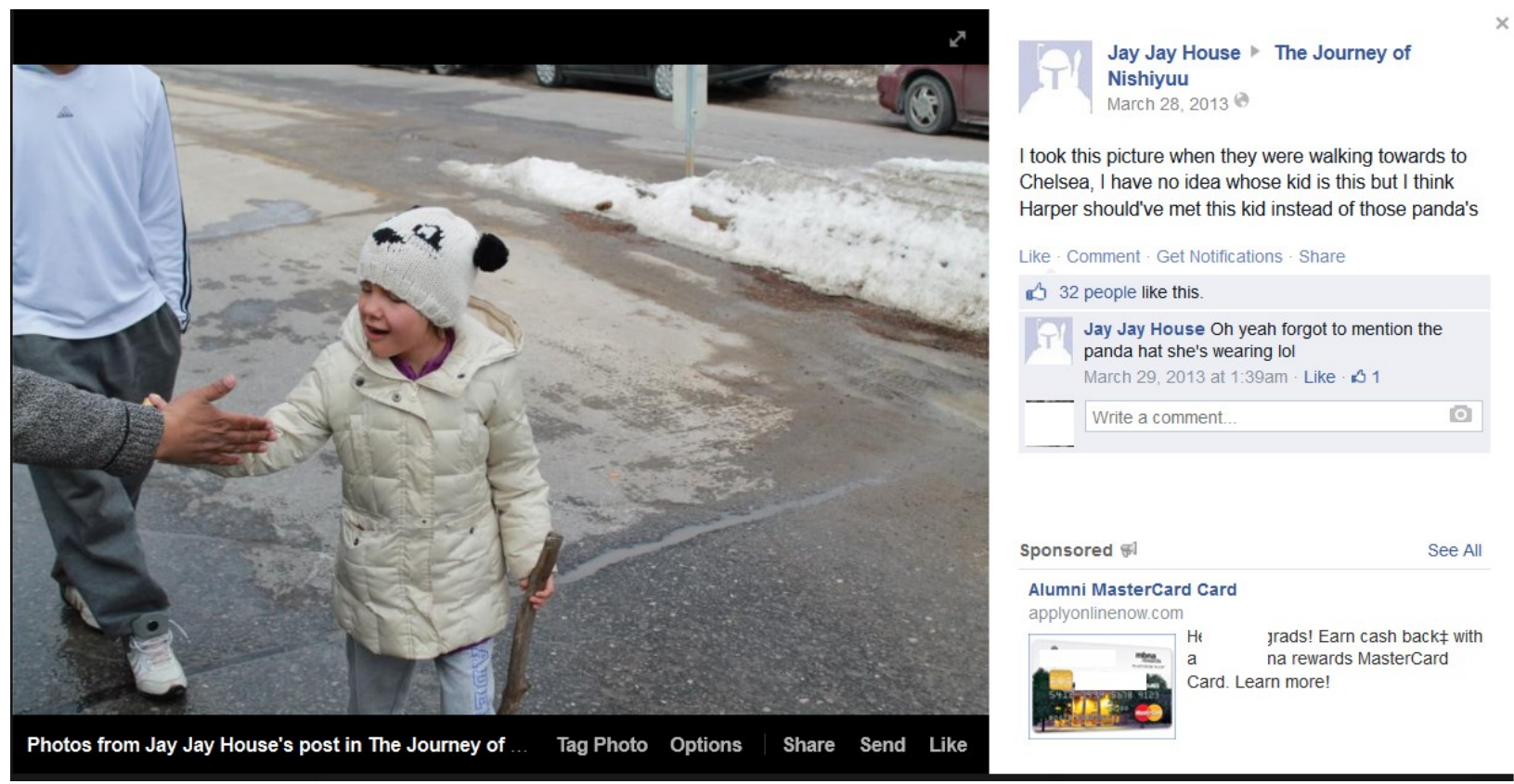

Figure 15: An image posted to the JON Facebook group page ${ }^{413}$. Shows an image in Facebook Theatre as presented on the desktop web. As seen from the caption, this photo shows a portion of the walk near Ottawa. The first comment refers to the panda hat on the child (drawing a connection to the Prime Ministers action on the day of the protest). This shows that reference and connection using digital communications can be multimodal and indirect.

413 Jay Jay House (March 28 2013) 


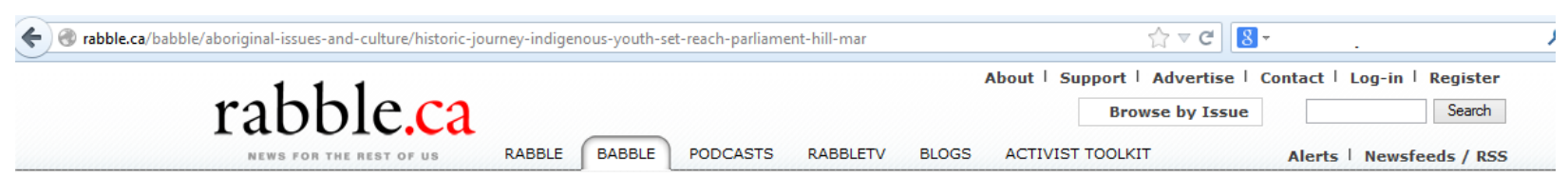

Forum Topics I Active Topics I Babble Policy

\section{JOIN THE GOOD JOBS REVOLUTION. \#Goodigbser \\ (U) UNIFOR \\ theUnion | lesyndicat}

babble babble is rabble.ca's discussion board but it's much more than that: it's an online community for folks who just
won't shut up. It's a place to tell each other - and the world - what's up with our work and campaigns.

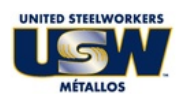

Historic Journey of Indigenous youth set to reach Parliament Hill March 25

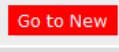

(1) Write to editor $\checkmark$ Support rabble

derrick

March 17, $2013-7: 59 \mathrm{pm}$

Joined: May 82008

Under the mainstream media's radar, an historic journey is one week away from completion. The plan right now is for a welcoming party March 25 on Parliament Hill. The group has grown from less than 10 to 200 people, along this journey of more than 1500 kilometres. Here is more information

from their website.

A journey of mythic proportions is being undertaken by six very courageous Cree Youth under 20 yrs old and one very wise Guide. They are walking from Whapmagoostui in Northern Quebec. Many other First Nations Youth are joining them along the way from the Cree \& Algonquin communities in their path.

More information about the journey can be found at: www.NishiyuuJourney.ca

The Journeyers are expected in Ottawa (Victoria Island) at 12 Noon on March 25th and celebration preparations are underway to welcome them.

Area schoolchildren ages 9-18 from the Ottawa District School Board, Kitigan Zibi, Akwesasne, \& Pikwakanagan are invited to walk the last part of the The Journey of Nishiyuu. At Victoria Island the hundreds of kids will stop to sample traditional soups \& stews in huge pots over little fires \& munch bannock. A ceremony to honour and welcome the Journeyers is to be held by the sacred fire of the Algonquins. They'll walk the final steps to Parliament Hill together. At the Hill, the Journeyers will share their messages, hear performers and Elders.

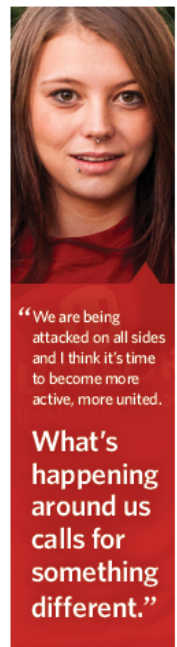

(U) UNIFOR Unionism

Figure 16: A screenshot of rabble.ca ${ }^{414}$. This shows a user posted entry into the discussion forum commenting on the lack of mainstream media coverage leading up to the JON's arrival in Ottawa. Content and links to the JON website are shared.

414 'derrick' (March 17 2013) 
Protests are here to stay everyday. Shouldn't the Cree be in school?

So I'm with Harper on the Panda visit.

It was important show of respect to China and good for business. We need to pay our aboriginal bills you know.

25 MAR 2013 5:04 PM

क REPLY \& FAVE ¿ SHARE *.. MORE

\section{redthunder}

Hey Retro Dude, way to show what you consider important in life, to be the human livestock which belongs to the British Monarch. Keeping on sending your tax dollars to all the politicians that pickpocket our spare cash each year. Don't forget to include all the politicians pensions for the measley 6 years or service to their employers

25 MAR 2013 5:10 PM

क REPLY \& FAVE $\angle$ SHARE $\ldots$ MORE

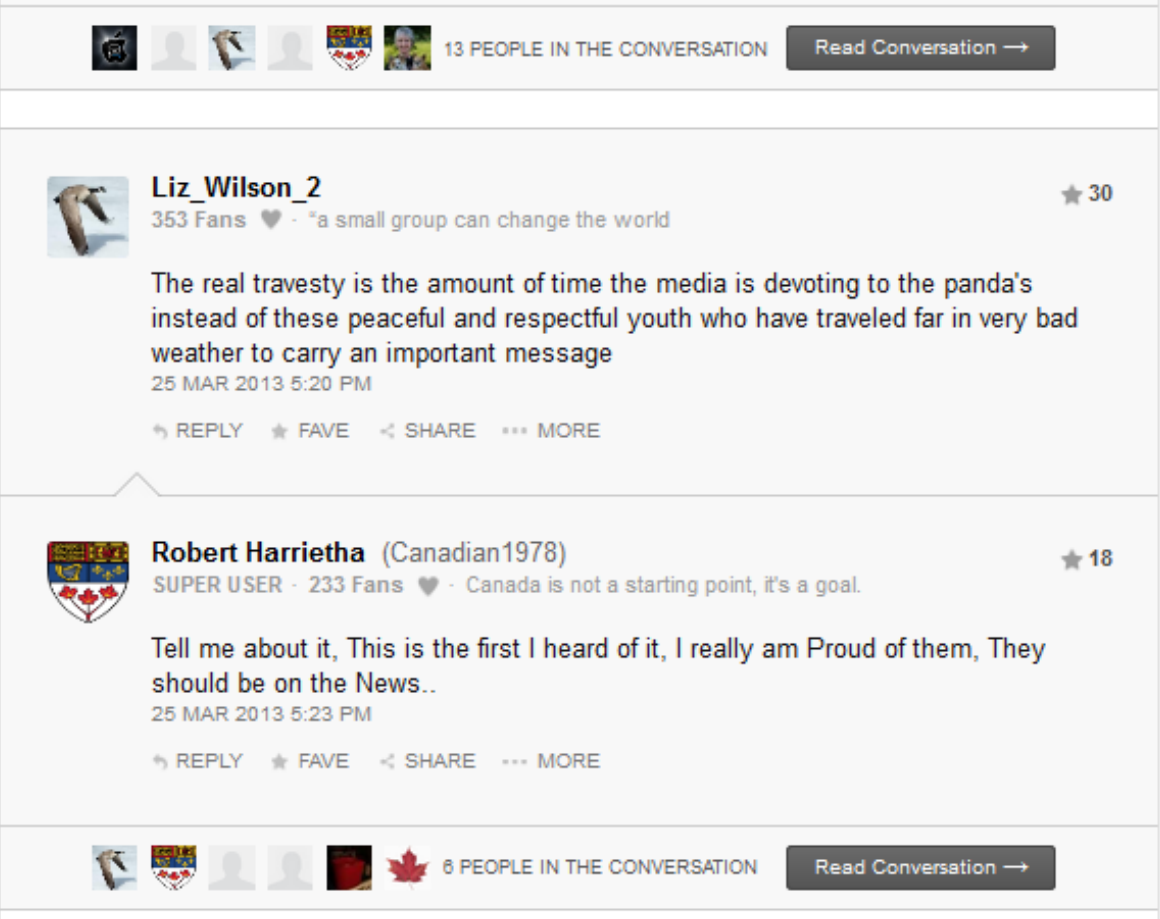




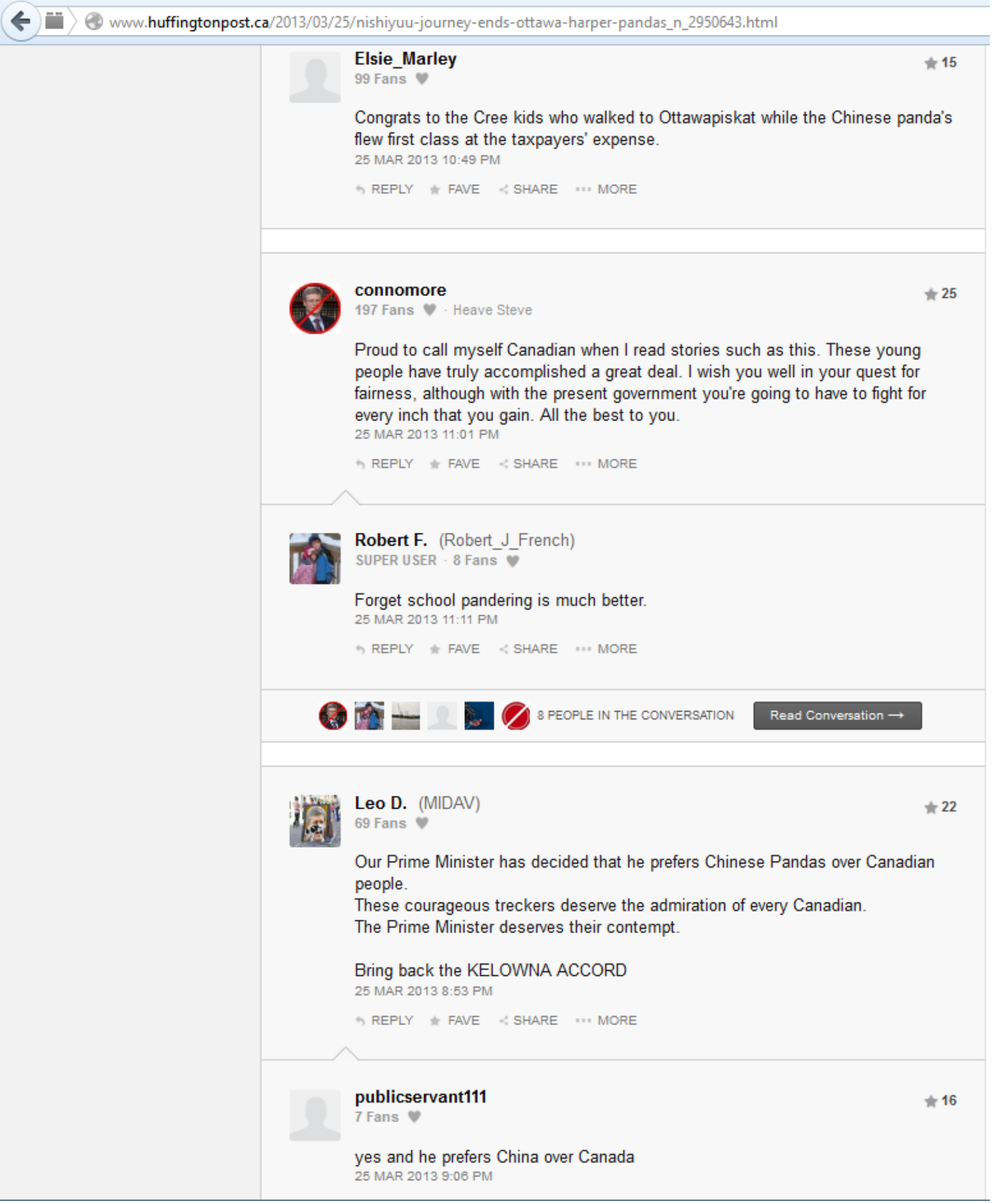

Figure 17: Comments section from Huffington Post article ${ }^{415}$. Profile pictures add context to user comments.

${ }^{415}$ Strapagie, Lauren (March 25 2013) 


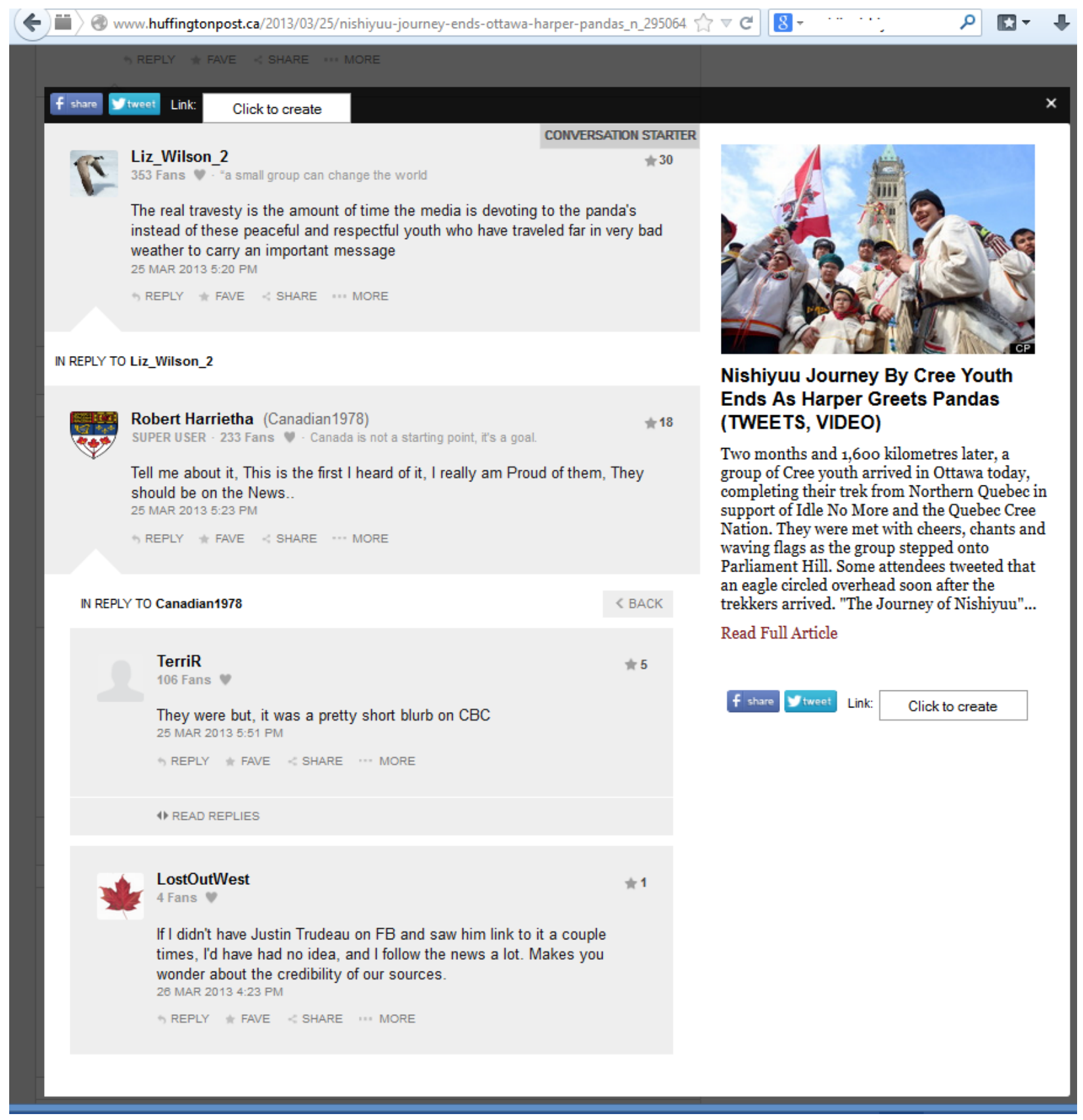

Figure 18: Conversation Thread remarking on media coverage ${ }^{416}$. User alludes to being initiated into the conversation from Social Media.

${ }^{416}$ Liz_Wilson_2 (March 25 2013) 


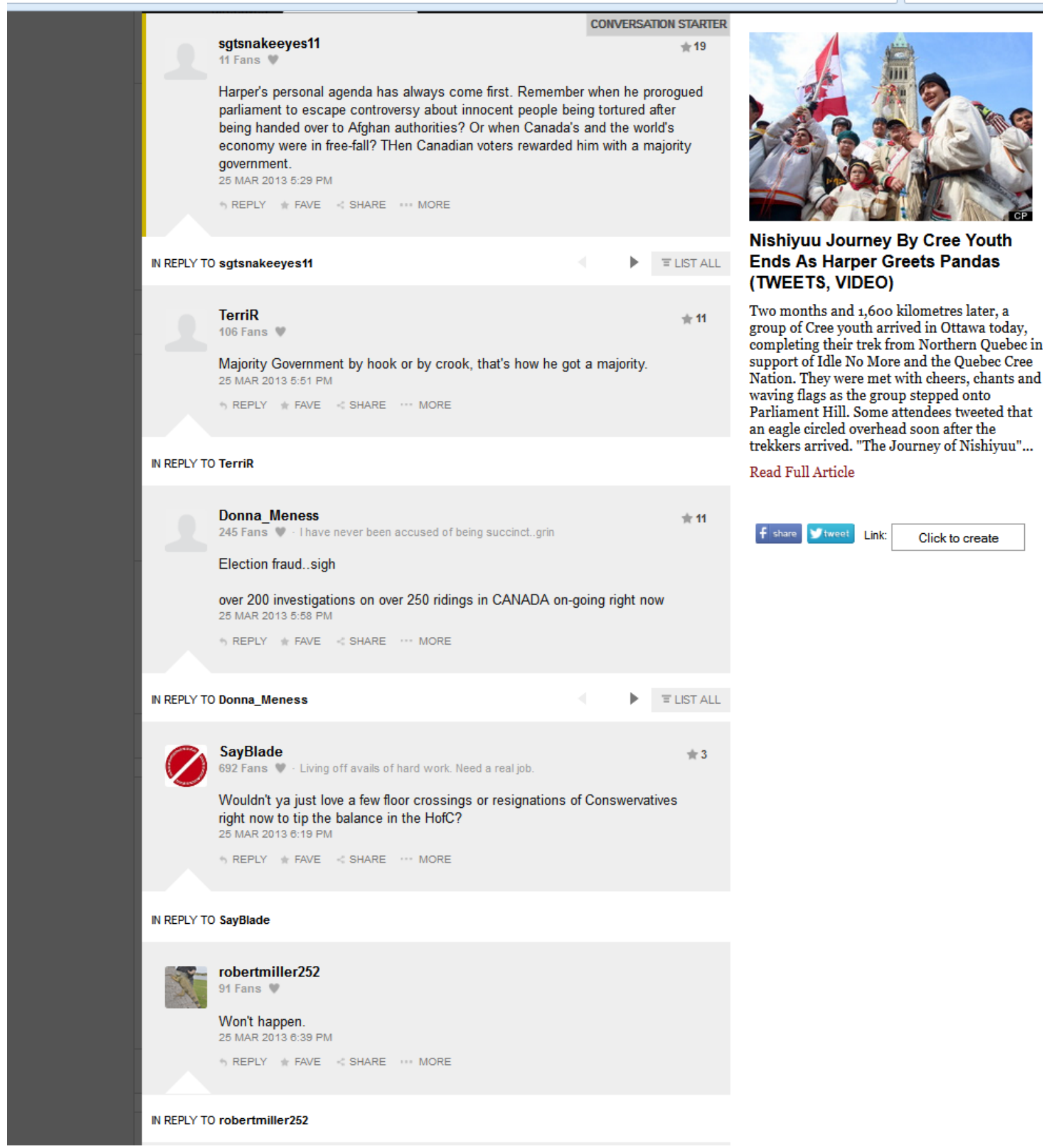

Figure 19: A conversation thread from Huffington Post ${ }^{417}$. Showing JON as an instigator for comments about politics tangential to the protest itself

${ }^{417}$ Srgtsnakeeyes11 (March 25 2013) 
Last accessed: March 30 2014 Last accessed: March 42014

\section{Google books Ngram Viewer}

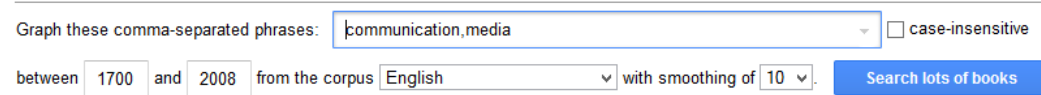

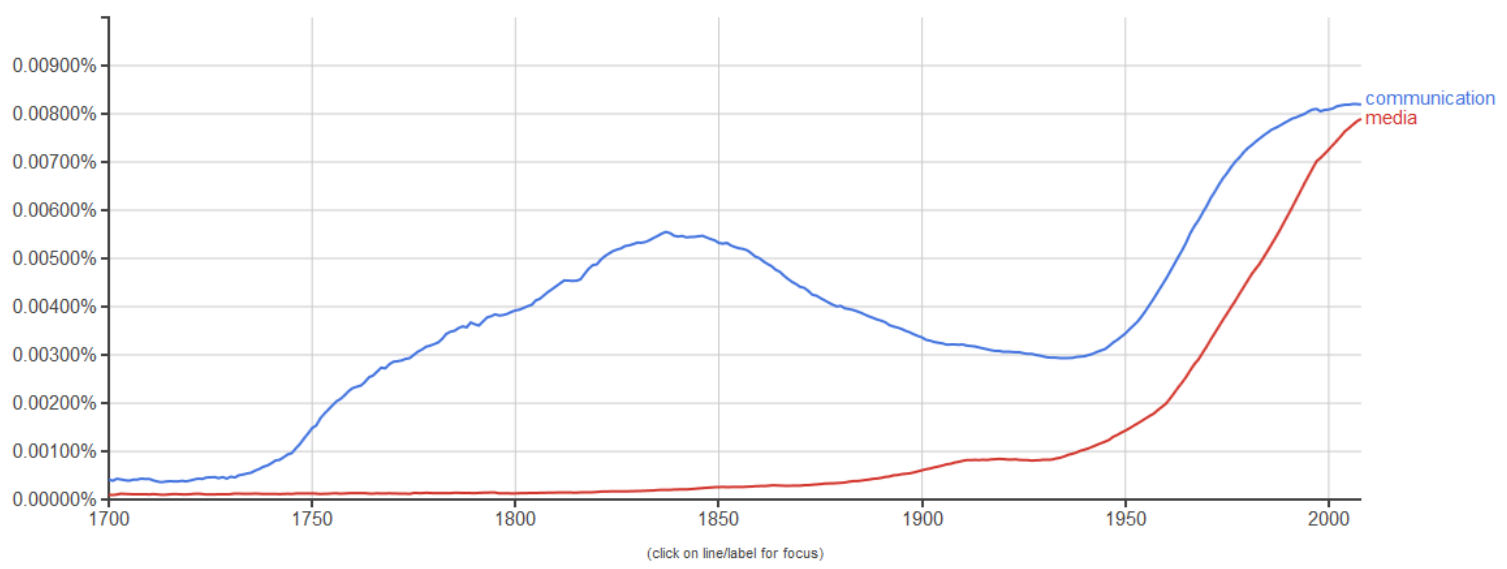

Search in Google Books

$\begin{array}{lllllll}1700-1784 & \underline{1785-1958} & \underline{1959-1978} & \underline{1979-1993} & \underline{1994-2008} & \underline{\text { communication }} & \text { English } \\ \underline{1700-1892} & \underline{1893-1995} & \underline{1996-2000} & \underline{2001-2004} & \underline{\underline{2005-2008}} & \underline{\text { media }} & \text { English }\end{array}$

Run your own experiment! Raw data is available for download here

๑ 2013 Google - Privacy \& Terms - About Google - About Google Books - About Ngram Viewer

Figure 20: An image rendered from Google Ngrams showing mentions of 'media' and 'communications in books since $1700^{418}$

\section{${ }^{418}$ https://books.google.com/ngrams}




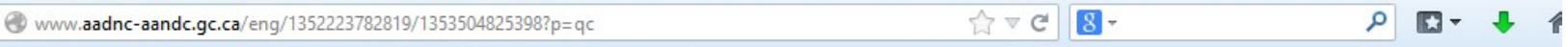

Aboriginal Connectivity Profiles of Quebec - As of March 2013

Current Residential Connectivity Availability

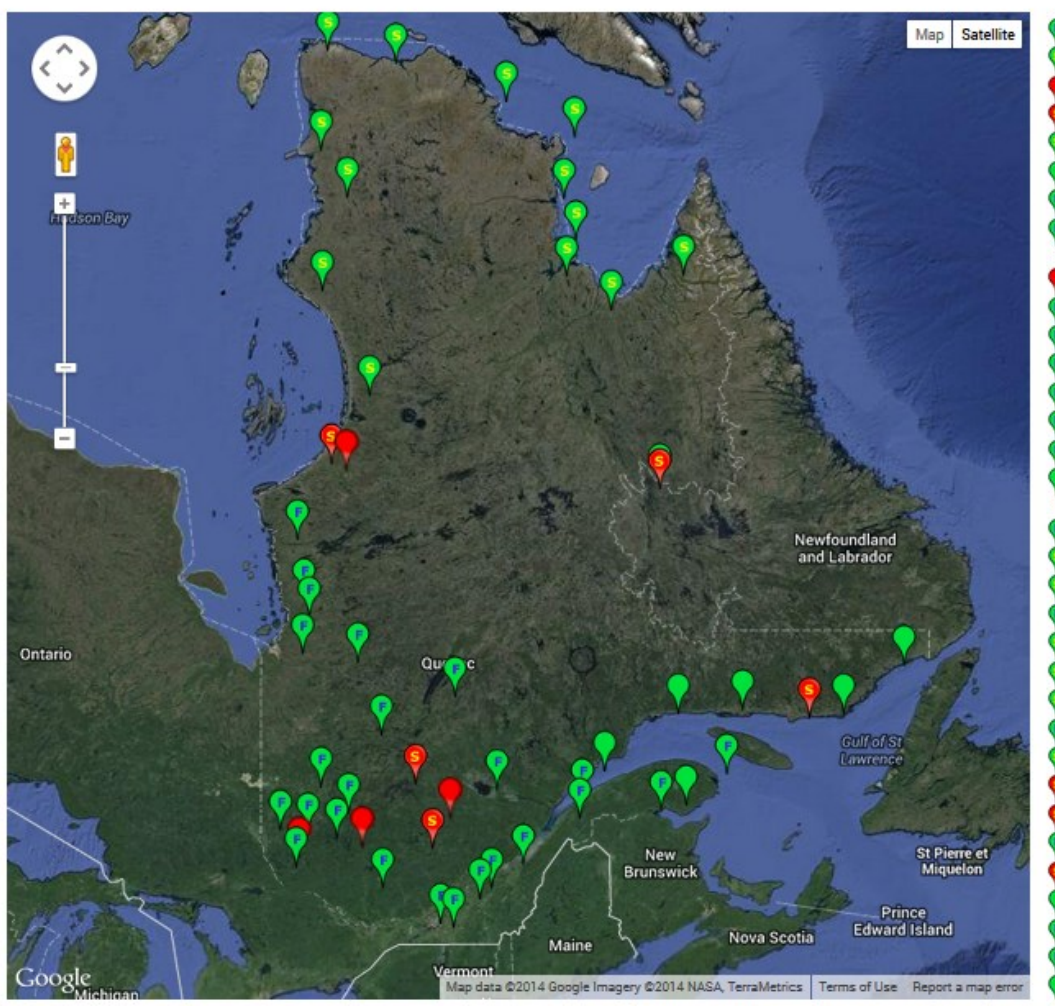

Abénakis de Wôlinak

Akulivik

Alqonquins of Barriere Lake

Atikamekw d'Opitciwan

Aupaluk

Bande des Innus de Pessamit

Communauté anicinape de Kitcisakik

Conseil de la Première Nation

Abitibiwinni

Conseil des Atikamekw de Wemotaci

Cree Nation of Chisasibi

Cree Nation of Mistissini

Cree Nation of Nemaska

Cree Nation of Wemindi

Eaqle Village First Nation - Kipawa

Eastmain

Innu Takuaikan Uashat Mak

Innue Essipit

Inukjuak

Ivuiivik

Kahnawake

Kanqiqsualuijuag

Kanqiqsuiuag

Kanqirsuk

Kitigan Zibi Anishinabed

Kuniiuag

Kuniiuarapik

La Nation Innu Matimekush-Lac John

La Nation Micmac de Gespeq

9 Les Atikamekw de Manawan

Les Innus de Ekuanitshit

Listuqui Mi'amaq Government

Long Point First Nation

Micmacs of Gesqapeqiag

Click on a coloured icon to view a short Connectivity Profile about the community.

Click on the name of a community to access a full Connectivity Profile.

Legend

Low Speed (Not connected as per Industry Canada minimum of $1.5 \mathrm{Mb} / \mathrm{s}$ to the household)

Consumer Broadband - Satelitemte (Not connected as per Industry Canada minimum of $1.5 \mathrm{mb} / \mathrm{s}$ to the household)

Industrial/Institutional Capable Broadband (Not connected. No residential access)

Consumer Broadband - Terrestrial (Connected as per Industry Canada minimum of $1.5 \mathrm{Mb} / \mathrm{s}$ to the household)

Consumer Broadband - Satelitemte (Connected as per Industry Canada minimum of $1.5 \mathrm{Mb} / \mathrm{s}$ to the household)

Industrial/Institutional Capable Broadband (Connected)

The Aboriginal Connectivity Profiles have been developed with the support of multiple stakeholders through a data collection process and methodology. Read the Data Methodology and Connectivity Glossary of Terms.

Figure 21: Map showing connectivity profiles of Aboriginal Communities in Quebec ${ }^{419}$. Produced by the government of Canada AADNC. The area the walkers originated from belongs is shown to have Industrial/Institutional Capable Broadband

${ }^{419}$ AANDC (March 2013) 
We encourage all readers to share their views on our articles and blog posts. We are committed to maintaining a lively but civil forum for discussion, so we ask you to avoid personal attacks, and please keep your comments relevant and respectful. If you encounter a comment that is abusive, click the " $X$ " in the upper right corner of the comment box to report spam or abuse. We are using Facebook commenting. Visit our FAQ page for more information.

\section{7 comments -}

\begin{tabular}{|c|c|c|c|}
\hline Add a comment... & & & \\
\hline$\checkmark$ Also post on Facebook & Posting as & I (Not you?) & Comment \\
\hline
\end{tabular}

www.ottawacitizen.com/Nishiyuu+Walkers+reach+Chelsea+their+ranks+swollen+from+seven+several+hundred/8145736/story,

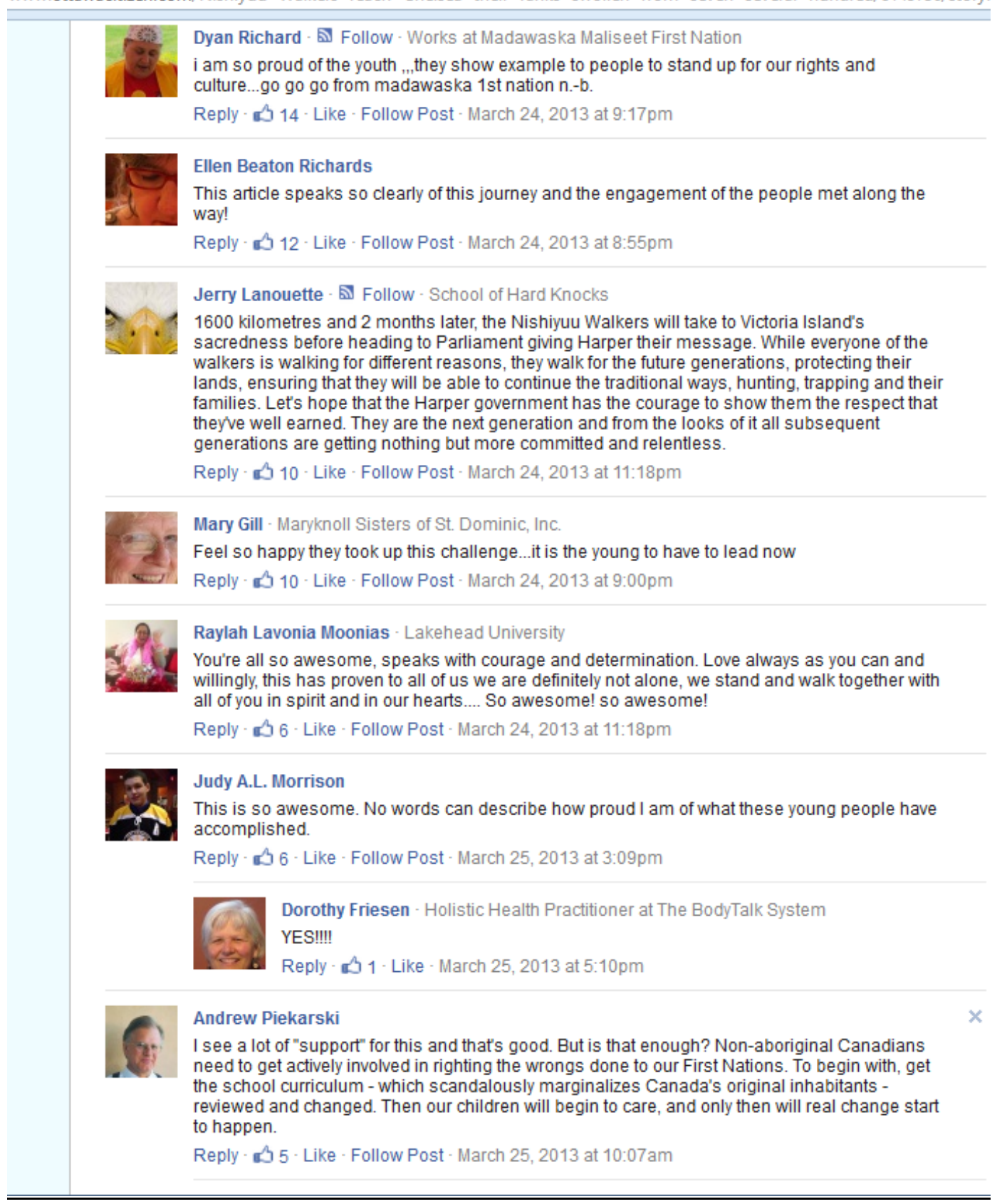




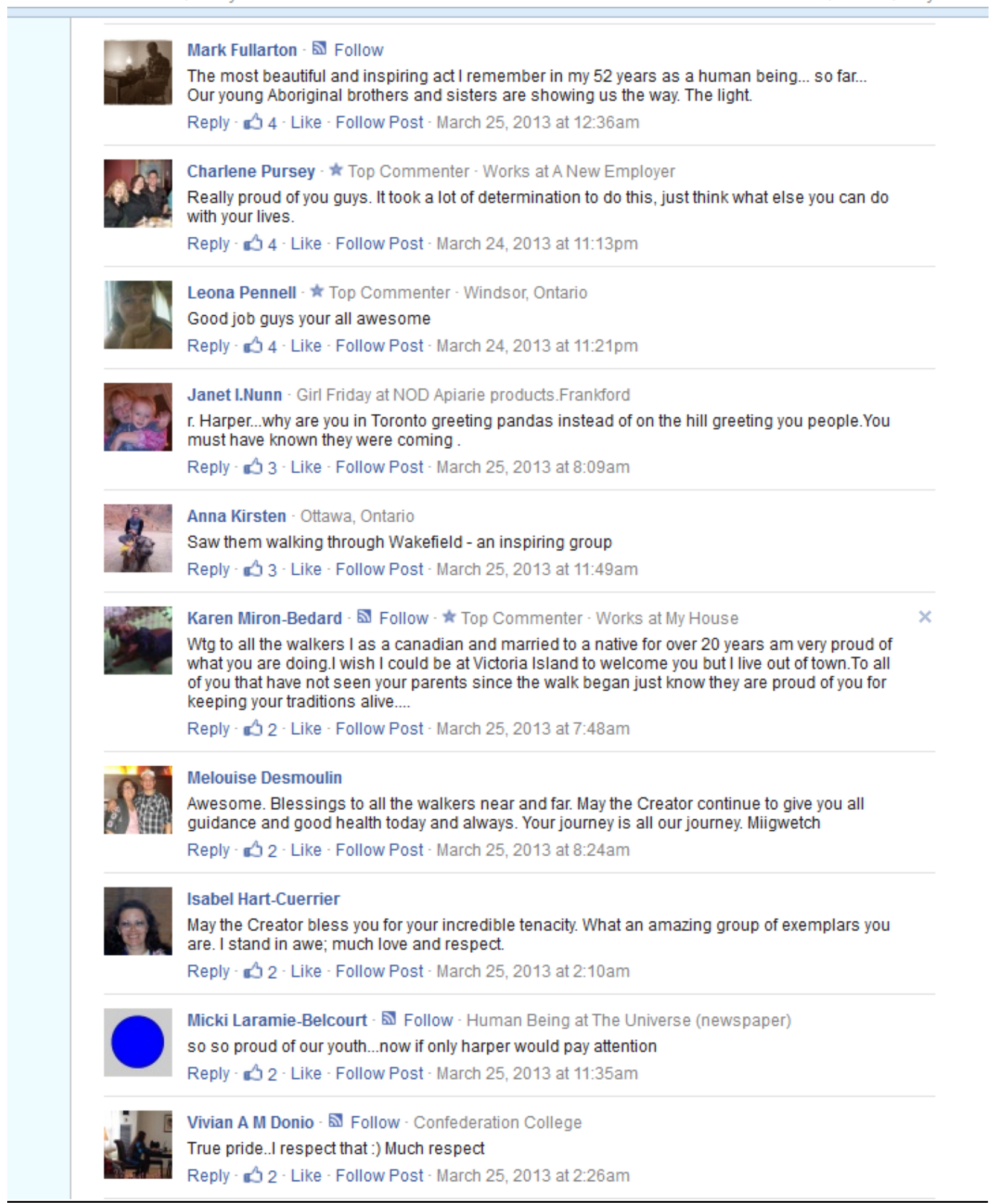

Figure 22: Comments section from an Ottawa Citizen News Article ${ }^{420}$. Including the preamble setting guidelines for appropriate discussion. This page uses Facebook for users to sign in and chat so profile picture and names are attached to comments.

420 Pearson, Matthew (March 25 ${ }^{\text {th }}$ 2013); From Comments section 
Congratulations. Now put this same energy into going after your corrupt band leaders and maybe the native problems will begin to be resolved.

$\underline{2}$ replies

Report Comment

\section{Algonquintaxpayer 370 days ago}

Just like Canadians oust all of their corrupt politicians too, right? Real life is not that simple.

Report Comment

Mikey from the GWN 370 days ago

"Just like Canadians oust all of their corrupt politicians too, right?"

Mulroney - gone

Cretien - gone

McGuinty - gone

etc etc

Just takes a little effort - try it some time!

Report Comment

\section{D_Peters 371 days agd}

How is walking to Ottawa a form of voting? Its not like they are taxpayers that can "vote" by moving to another jurisdiction to protest taxes in another. They get paid anyways, no taxes either.

1 reply

Report Comment

\section{Algonquintaxpayer 370 days ago}

First off, these were kids who started the march, so being a taxpayer or not has nothing to do with it. It is a show of strength and solidarity in the face of a government unwilling to meaningfully address native issues. Secondly, A large majority of First Nations people do pay property and income tax just like every other Canadian. And please, let's not act like First Nations people living on reserve are the only people in Canada who get tax exemptions. They are people who have as much a right to vote or protest as anyone else in this country.

Report Comment

Congratulations young folk. Peace be with you . 
it's a great publicity stunt, but unless they can convert it into more participation in education or employment, just a flash in the pan

Report Comment

Liberal cash envelopes 371 days ago

So how did these marchers get time off work to do this march?

$\checkmark \underline{5 \text { replies }}$

Report Comment

Needreason 371 days ago

Okay that's funny.

Report Comment

chimpdaddy 371 days ago

I imagine that in many cases there were neither jobs available nor people willing to hire young native people with little education. Economic development is pretty non-existent especially in the North. It's a bit rich to suggest that people who have walked thousands of kilometres are lazy, though, if that's where you are going.

Report Comment

Xondar 370 days ago

And does your boss give you time off so you can search fore your honour and integrity?

Report Comment

Voice O'Reason 370 days ago

They have direct deposit.

Report Comment

lilmissthink 370 days ago

yes, chimpdaddy, which is why no matter how many miles you walk, nothing will change. Like everyone else in the world, FN people must move to where the jobs are, or be left behind.

Report Comment

Figure 23: An example of a text-only comments section. A more anonymous comments section from the Globe and Mail421. Amidst supporters there are more dissenting views expressed. Also some posts are replies to user's comments, not the article

${ }^{421}$ Galloway, Gloria (March 25 ${ }^{\text {th }}$ 2013); From Comments section 
Has anyone bothered to check this story out or is it phony like chief many chins hunger strike.About 960 miles in approx. 65 days straight, no time off is about 14 miles a day.Where did they stay?Were any vehicles involved?Where did they eat the energy needed to travel those miles in cold weather would be a huge consideration. if they did it good for them, but if it was a scam like many chins protest the readers should be told.

- 21 Replies

Spaz_td - 1 year 0 months ago $\mid$ Report Abuse

$=\mathbb{A}$ chief many chins... hilarious! lol

\section{3 [圈 8}

8

SkeptiChris - 1 year 0 months ago | Report Abuse

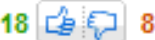

and more to the point.. if they did walk that far.. why not put that effort into

bettering your community yourself? the rest of us do. it's called a society.

A Yahoo User - 1 year 0 months ago

18 [슬 11

why are these 'walkers' not in school or at work?

2

Pickle - 1 year 0 months ago | Report Abuse

14 [色包 8

It undoubtably was a scam. You will notice there were no pictures showing the entire walk. It was another chief many chins fiasco.

Schlomo - 1 year 0 months ago | Report Abuse

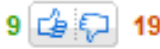

What sad people you all are. A group of youth accomplish something that you couldn't get your fat azzes off the sofas to even imagine trying, so it must be a scam or a con. Your comments are just more proof positive that no matter what, your minds are made up about native people They camped, there are no 'vehicles' to help them out in the bush and yes, the trip is recorded and CBC news checked on othem often. Grow up. "no pictures showing the entire walk?" Like you have the capacity to sit and view that many pictures? Give us all a break.

Elle Ryder Boom - 1 year 0 months ago | Report Abuse

5 놉 1

Man, Shut Up!

Eric - 1 year 0 months ago $\mid$ Report Abuse

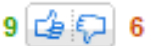

@schlomo your a tool damn right i couldn't accomplish it, i would never be able to take that amount of time off my full time salary position. Why don't you go $f$ yourself and get a job douchebag. 
I'm sorry, you're wrong in characterizing us all as having made our minds up about the First Nations. I want to see fair treatment of FN, but there is quite a bit that they can do for themselves as well. They are afforded luxuries that no one else gets.

They live in an entitled micro-society within greater Canada. They are not empowered, they are entitled. The federal government has to save them from themselves, over and over again. It's not a racist thing.. I've known FN living off reserve that make it in society. Their reserves do not operate like an incorporated small city. Their finances are not subjected to the same scrutiny.

It's a corrupt system. The feds leave them to their own devices (have done so for decades). Native tribal politics is more like family politics rather than town politics

They should be given every right, privelege, AND responsibility that every other canadian has. The problem is they want to police themselves. They want their own schools. and when those police don't stop the crime, and when their schools fail them, they ask the feds for more money to fix the problem.. half of which pads the pockets of the monarchy... erm.. I mean, the band council.

Walter B - 1 year 0 months ago | Report Abuse

Many chins are less of a burden than few brains. Too many here put their fingers in play before their brains are engaged.

SkeptiChris - 1 year 0 months ago $\mid$ Report Abuse

Walter,

thank you for giving us your view without actually contributing to the discussion

Schlomo - 1 year 0 months ago | Report Abuse

eric, thanks for sharing your ability to insult people without actually contributing anything intelligent to what was said. More evidence that the intellectually challenged will always lower themselves to insults before coming up with anything logical to say.

Schlomo - 1 year 0 months ago | Report Abuse

Chris, if you're not one of the people I referred to, then good for you. Then there is no need to respond. There is, believe me, neither entitlement nor empowerment on reservations or other Aboriginal communities, nor is there any racial parity in our cities, as much as mainstream society will deny it. You started your points of quite well, but had to lapse into the usual nonsense about the 'monarchies' at the end. Even the comment, "the feds leave them to their own devices" The fact is that if the feds had stopped meddling in the Indians' business decaded ago, these problems would not exist. These people were fully capable of self governance for millenia prior to European interference. I must say, that at least you are capable of intelligent response which is a far better thing than most of the people l've had the displeasure of trying to reach out to on this subject matter. After a while I find myself starting to lower myself to their denominator of insult and anger and should try to avoid that.

Schlomo - 1 year 0 months ago | Report Abuse

Elle, brilliant response, did you choose that because writing "OH YEAH?" might have made you look stupid? 
Schlomo.. I want to rename you Schlolo.. sorry.. had to write that.

yeah you seem like you can debate well yourself.

I admit that natines managed just fine before the whitey came around, but tell me, what is it about today's world that doesnt allow for them to self-govern to the standards of our society?

back then, they had no fall back.. they hunted, they fished, they built their own homes,... etc

today, they could easily live in that way.. they could hunt and fish, build their own homes (historical or modern).. there are plenty of people out there wanting to buy native art, which can fetch a pretty penny too.

I can't help but think that there are reserves that do well, and yet there are others that do not. there must be a reason for this.

and my invoking of the "monarchy" isn't far from the truth. a reserve is made up of one or more clans, and as such, you get family politics. I don't mean to make it sound feudalistic,.. the chief's son isn't going to put a knife in his father's back.. but I really do wonder what the voting structure is for these band councils.

in FN populations, you get higher incidences of criminal behaviour, of drug dependency.. things like this. I suspect that it is easier to be a non productive member of society when you're on reserve. I couldn't begin to tell you the economics of it.

I don't percieve the federal government meddling with the affairs of the FN. if anything they are hands off. The problem in my mind is that the reserve isn't a pristine environment where natives live as they did in the 1700 s. they are modern towns with modern amenities like sewage and clean water. the feds have the responsibility to ensure that infrastructure stays working. I wonder how many natives on reserve actually know how to maintain these systems. how many of them actually do this on their own.

you hear reports of band council members taking home $\$ 200 \mathrm{k}$. and wonder.

SkeptiChris - 1 year 0 months ago | Report Abuse $3\left[\begin{array}{l}\text { [ } \\ 1\end{array}\right.$

but at the end of the day there's a logical flaw in your argument.. they were fine before europeans came here,.. but now when they need help they walk $1500 \mathrm{~km}$ to ask the fed for help? there's a disconnect there. they want more from the government,... more assistance.. more what ever. they arent putting up fences and guarding their borders from the federal government coming to them and interfering with their lives. they get cashflow from the federal government. they really do live in the best of both worlds.. and yet many live in squalor.

Figure 24: A lively (and adversarial) back and forth discussion between users on Yahoo News ${ }^{422}$. This is an excerpt from a thread of 21 comments replying to a user "Terry" who questioned whether the walk was a hoax. Several users comment, but ultimately two users exchange lengthy replies back and forth.

422 Yahoo! Canada News (March 26, 2013) 


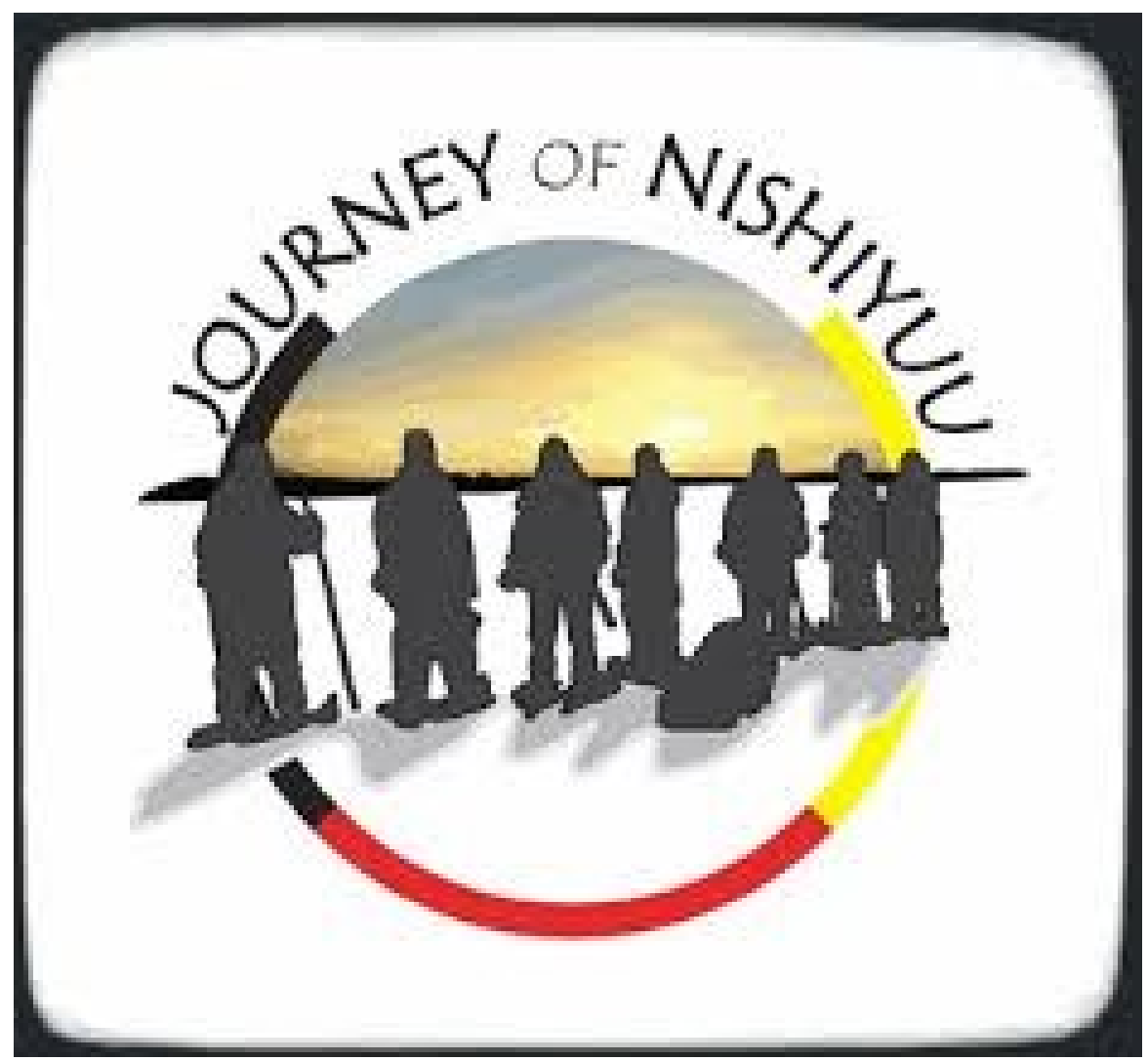

Figure 25: JON logo featured on the main website, but also shared by users on image sharing sites like Pinterest to link to the website $^{423}$

423 “Logo" (January 6 $6^{\text {th }} 2013$ ) 


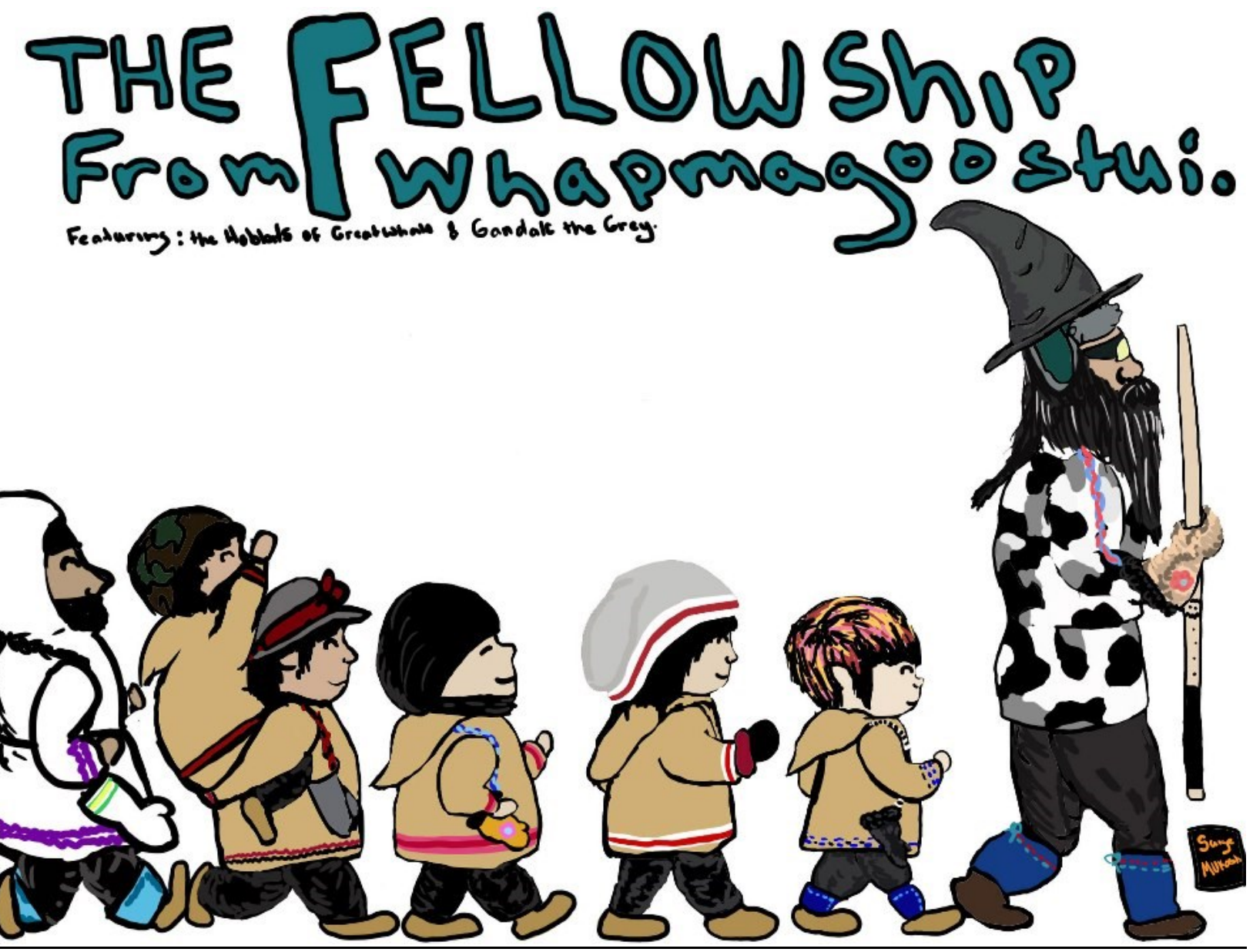

Figure 26: A tablet-drawn image comparing the JON to the Lord of the Rings Trilogy ${ }^{424}$. This image was found on a blogspot page, but is attributed to a post on the JON Facebook Group page.

${ }^{424}$ Mukash, Saige (January 23, 2013) 


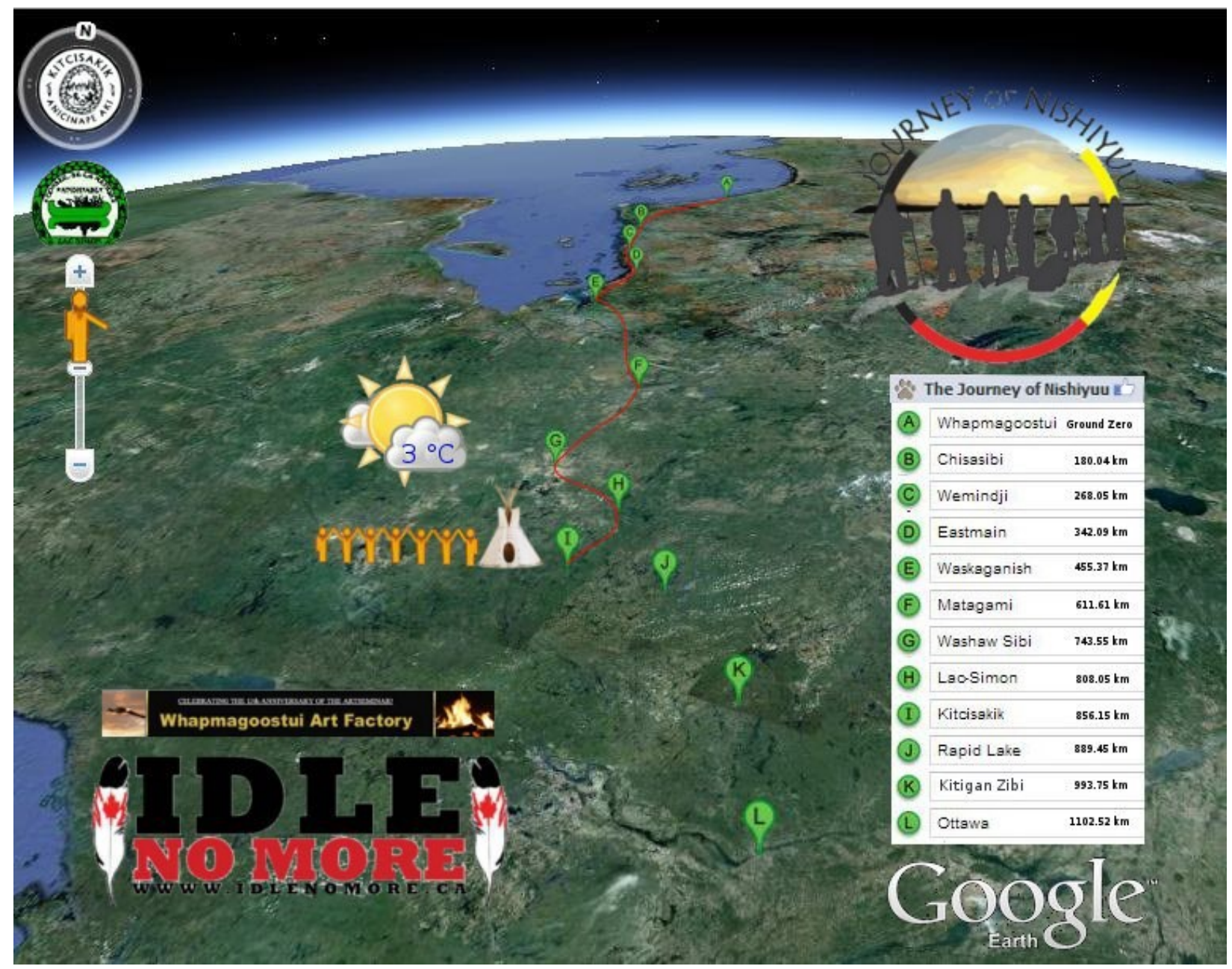

Figure 27: Image of Google Earth map with points demarcating the JON trajectory ${ }^{425}$. The red line indicated the path of the walkers. Image is digitally created (Using Google Earth) and altered.

${ }^{425}$ Gups-Y-Bees Lepled (March 9, 2013) 


\section{C.2 \#Nishiyuu Tweets}

\begin{tabular}{|c|c|c|c|c|c|c|c|c|}
\hline User & Tweet & $\begin{array}{l}\text { Date } \\
\text { and } \\
\text { Time }\end{array}$ & Retweets & Favourites & $\begin{array}{l}\text { Direct } \\
\text { Replies }\end{array}$ & Type & $\begin{array}{l}\text { Journalist } \\
\text { or } \\
\text { Politician }\end{array}$ & $\begin{array}{l}\text { Associated } \\
\text { Tweets }\end{array}$ \\
\hline $\begin{array}{l}\text { Ian Stephen } \\
\text { @lanstephen1 }\end{array}$ & $\begin{array}{l}\text { Drummers stopped and in } \\
\text { tears as lone voice sings. } \\
\text { \#nishiyuu } \\
\text { http://www.livestream.co } \\
\text { m/occupytoronto }\end{array}$ & $\begin{array}{l}2013- \\
03-25 \\
11: 21\end{array}$ & 2 & 0 & 0 & $\begin{array}{l}\text { Hyperlink } \\
\text { to Online } \\
\text { Video }\end{array}$ & $\mathrm{j}$ & 197 \\
\hline $\begin{array}{l}\text { jian ghomeshi } \\
\text { @jianghomeshi }\end{array}$ & $\begin{array}{l}\text { The \#Nishiyuu walkers are } \\
\text { a true inspiration. And } \\
\text { deserve more attention } \\
\text { than visiting pandas. - my } \\
\text { opening essay tmrw morn. } \\
\text { \#Q }\end{array}$ & $\begin{array}{l}2013- \\
03-25 \\
15: 15\end{array}$ & 357 & 102 & 4 & Post & $\mathrm{j}$ & 385 \\
\hline $\begin{array}{l}\text { CBC Top Stories } \\
@ \text { cbcnews }\end{array}$ & $\begin{array}{l}\text { Cree youth Idle No More } \\
\text { trek from Northern Quebec } \\
\text { to end in Ottawa today } \\
\text { http://cbc.sh/UlfUpbk } \\
\text { \#Nishiyuu }\end{array}$ & $\begin{array}{r}2013- \\
03-25 \\
6: 21\end{array}$ & 130 & 14 & 5 & $\begin{array}{l}\text { Embedded } \\
\text { News } \\
\text { Article/ } \\
\text { Online } \\
\text { Video }\end{array}$ & j & 203 \\
\hline $\begin{array}{l}\text { CBC Top Stories } \\
@ \text { cbcnews }\end{array}$ & $\begin{array}{l}\text { Cree Idle No More walkers } \\
\text { near end of 1,600 km trek } \\
\text { to Ottawa. } 7 \text { started in N. } \\
\text { Quebec. Now close to } 200 . \\
\text { http://cbc.sh/QOFoX1E } \\
\text { \#Nishiyuu }\end{array}$ & $\begin{array}{l}2013- \\
03-22 \\
12: 44\end{array}$ & 140 & 14 & 9 & $\begin{array}{l}\text { Embedded } \\
\text { News } \\
\text { Article/ } \\
\text { Online } \\
\text { Video }\end{array}$ & j & 136 \\
\hline @Michelle9647 & $\begin{array}{l}\text { \#FacePalm Harper greets } \\
\text { pandas while \#Nishiyuu } \\
\text { walkers return } \\
\text { \#IdleNoMore \#cdnpoli } \\
\text { http://huff.to/14jOGT6 " }\end{array}$ & $\begin{array}{l}2013- \\
03-25 \\
14: 03\end{array}$ & 2 & 1 & 0 & $\begin{array}{l}\text { Embedded } \\
\text { News } \\
\text { Article/ } \\
\text { Online } \\
\text { Video }\end{array}$ & j & 316 \\
\hline $\begin{array}{l}\text { Jorge Barrera } \\
\text { @JorgeBarrera }\end{array}$ & $\begin{array}{l}\text { Another shot the crowd. } \\
\text { Journey of \#Nishiyuu } \\
\text { http://yfrog.com/odhfqhoj }\end{array}$ & $\begin{array}{l}2013- \\
03-25 \\
11: 16\end{array}$ & 15 & 3 & 3 & Photo & j & 59 \\
\hline $\begin{array}{l}\text { Amanda T. Sam } \\
\text { @ouwaah }\end{array}$ & $\begin{array}{l}\text { Bob Marley's } \\
\text { granddaughter walking } \\
\text { with \#nishiyuu } \\
\text { http://instagram.com/p/XS } \\
\text { zQAituA5/ }\end{array}$ & $\begin{array}{l}2013- \\
03-25 \\
13: 24\end{array}$ & 56 & 14 & 4 & Photo & no & 77 \\
\hline $\begin{array}{l}\text { Eli D. M. } \\
\text { @eli_d_m }\end{array}$ & $\begin{array}{l}\text { Sad day today, Isaac } \\
\text { Kawapit has passed away.. } \\
\text { He guided the \#Nishiyuu } \\
\text { walkers to Ottawa.. } \\
\text { \#Eeyoulstchee RIP }\end{array}$ & $\begin{array}{l}2013- \\
07-14 \\
10: 08\end{array}$ & 41 & 4 & 2 & Photo & no & 42 \\
\hline
\end{tabular}




\begin{tabular}{|c|c|c|c|c|c|c|c|c|}
\hline & $\begin{array}{l}\text { pic.twitter.com/oLumiERO0 } \\
\text { B }\end{array}$ & & & & & & & \\
\hline $\begin{array}{l}\text { Jorge Barrera } \\
\text { @JorgeBarrera }\end{array}$ & $\begin{array}{l}\text { Steps away from } \\
\text { Parliament Hill, chants of } \\
\text { "Harper, Harper." Journey } \\
\text { of \#Nishiyuu } \\
\text { http://yfrog.com/oe1evgij }\end{array}$ & $\begin{array}{l}2013- \\
03-25 \\
11: 04\end{array}$ & 14 & 2 & 1 & Photo & $\mathrm{j}$ & 20 \\
\hline $\begin{array}{l}\text { CBC Top Stories } \\
\text { @cbcnews }\end{array}$ & $\begin{array}{l}\text { Cree walkers finally arrive } \\
\text { in Ottawa on Idle No More } \\
\text { trek. Left Northern Quebec } \\
\text { in January. Watch LIVE: } \\
\text { http://cbc.sh/29PtZdl } \\
\text { \#Nishiyuu }\end{array}$ & $\begin{array}{r}2013- \\
03-25 \\
9: 53\end{array}$ & 82 & 12 & 1 & $\begin{array}{l}\text { Hyperlink } \\
\text { to News } \\
\text { Article/ } \\
\text { Online } \\
\text { Video }\end{array}$ & $j$ & 65 \\
\hline $\begin{array}{l}\text { The National } \\
@ \text { CBCTheNatio } \\
\text { nal }\end{array}$ & $\begin{array}{l}\text { Cree walkers have arrived } \\
\text { in Ottawa from Northern } \\
\text { Que. Here's Friday's story } \\
\text { \& more to come tonight. } \\
\text { http://www.youtube.com/ } \\
\text { watch?v=ivLqakosB-A ... } \\
\text { \#Nishiyuu }\end{array}$ & $\begin{array}{l}2013- \\
03-25 \\
10: 39\end{array}$ & 77 & 21 & 7 & $\begin{array}{l}\text { Embedded } \\
\text { Video }\end{array}$ & j & 82 \\
\hline $\begin{array}{l}\text { Justin Trudeau, } \\
\text { MP Verified } \\
\text { account } \\
\text { @JustinTrudeau }\end{array}$ & $\begin{array}{l}\text { The Journey of Nishiyuu } \\
\text { shows commitment to a } \\
\text { better future for FN } \\
\text { communities. Let's equal } \\
\text { that with real engagement. } \\
\text { \#idlenomore \#Nishiyuu }\end{array}$ & $\begin{array}{l}2013- \\
03-25 \\
10: 04\end{array}$ & 234 & 58 & 5 & Post & $p$ & 233 \\
\hline $\begin{array}{l}\text { Kai Nagata } \\
\text { @kainagata }\end{array}$ & $\begin{array}{l}\text { Elizabeth May, Nathan } \\
\text { Cullen and Joyce Murray } \\
\text { skipped QP to greet the } \\
\text { \#Nishiyuu walkers on the } \\
\text { steps of Parliament. An } \\
\text { eagle flew over. }\end{array}$ & $\begin{array}{l}2013- \\
03-25 \\
11: 49\end{array}$ & 109 & 22 & 8 & Post & j & 115 \\
\hline $\begin{array}{l}\text { rabbleca } \\
\text { @rabbleca }\end{array}$ & $\begin{array}{l}\text { \#Nishiyuu is trending in } \\
\text { Canada. Here's some } \\
\text { background about this epic } \\
\text { journey } \\
\text { http://rabble.ca/news/201 } \\
\text { 3/03/indigenous-youth- } \\
\text { epic-journey-ottawa- } \\
\text { deserve-attention-and- } \\
\text { respect ... \#ldleNoMore } \\
\text { \#cdnpoli }\end{array}$ & $\begin{array}{l}2013- \\
03-25 \\
12: 22\end{array}$ & 73 & 11 & 2 & $\begin{array}{l}\text { Hyperlink } \\
\text { to News } \\
\text { Article }\end{array}$ & j & 69 \\
\hline $\begin{array}{l}\text { Nishiyuu } \\
@ \text { Nishiiyuu }\end{array}$ & $\begin{array}{l}\text { \#Unity has been witnessed } \\
\text { on the \#Journey from all } \\
\text { walks of life! We thank you } \\
\text { with \#Love. \#Nishiyuu }\end{array}$ & $\begin{array}{l}2013- \\
03-24 \\
15: 38\end{array}$ & 60 & 16 & 3 & Post & no & 63 \\
\hline UBCIC @UBCIC & Harper chooses to greets & 2013- & 77 & 6 & 8 & Post, & $\mathrm{g}$ & 76 \\
\hline
\end{tabular}




\begin{tabular}{|c|c|c|c|c|c|c|c|c|}
\hline & $\begin{array}{l}\text { pandas instead of our } \\
\text { \#Nishiyuu walkers today } \\
\text { https://fbcdn-sphotos-d- } \\
\text { a.akamaihd.net/hphotos- } \\
\text { ak- } \\
\text { ash3/882928_1015130906 } \\
\text { 5315672_1234188797_o.jp } \\
\text { g ... }\end{array}$ & $\begin{array}{l}03-25 \\
12: 12\end{array}$ & & & & \begin{tabular}{|l|} 
Hyperlink \\
to Photo
\end{tabular} & & \\
\hline $\begin{array}{l}\text { CBC Top Stories } \\
\text { @cbcnews }\end{array}$ & $\begin{array}{l}\text { A shot of the \#Nishiyuu } \\
\text { walkers in Chelsea, Que., } \\
\text { from CBC's } \\
\text { @ryanpaulgibson. } \\
\text { pic.twitter.com/yicyS7qzgF } \\
\text { http://cbc.sh/UlfUpbk }\end{array}$ & $\begin{array}{r}2013- \\
03-25 \\
6: 20\end{array}$ & 48 & 7 & 2 & Photo & j & 47 \\
\hline $\begin{array}{l}\text { Jarrett } \\
\text { Martineau } \\
\text { @culturite }\end{array}$ & $\begin{array}{l}\text { "Every step I took was a } \\
\text { prayer for mother } \\
\text { earth...and all people } \\
\text { around the world." 11- } \\
\text { year-old \#Nishiyuu walker } \\
\text { \#INM }\end{array}$ & $\begin{array}{l}2013- \\
03-25 \\
12: 51\end{array}$ & 95 & 27 & 4 & Post & no & 91 \\
\hline Sarah@Sar_koi & $\begin{array}{l}\text { Bob Marley's } \\
\text { granddaughter stands with } \\
\text { \#inm \#Nishiyuu } \\
\text { pic.twitter.com/4ux6CH2fo } \\
\text { m }\end{array}$ & $\begin{array}{l}2013- \\
03-18 \\
11: 28\end{array}$ & 67 & 11 & 1 & $\begin{array}{l}\text { Photo (of } \\
\text { Facebook } \\
\text { Post) }\end{array}$ & no & 63 \\
\hline $\begin{array}{l}\text { Christi Belcourt } \\
\text { @christibelcour } \\
\mathrm{t}\end{array}$ & $\begin{array}{l}\text { If I was Prime Minister, I'd } \\
\text { be on Parliament Hill to } \\
\text { greet the } 300 \text { youth } \\
\text { leaders who walked } \\
1,700 \mathrm{~km} \text { to get there. } \\
\text { \#INM \#RISE \#Nishiyuu }\end{array}$ & $\begin{array}{r}2013- \\
03-25 \\
6: 20\end{array}$ & 60 & 17 & 5 & Post & no & 66 \\
\hline $\begin{array}{l}\text { Olivia Chow } \\
\text { @oliviachow via } \\
\text { @nathancullen }\end{array}$ & $\begin{array}{l}\text { A powerful scene on } \\
\text { Parliament Hill this aft to } \\
\text { welcome the \#Nishiyuu } \\
\text { walkers to Ottawa. } \\
\text { pic.twitter.com/8TwIEftcAR } \\
\text { via @nathancullen }\end{array}$ & $\begin{array}{l}2013- \\
03-25 \\
12: 05\end{array}$ & 88 & 14 & 7 & Photo & $p$ & 84 \\
\hline $\begin{array}{l}\text { Kai Nagata } \\
\text { @kainagata }\end{array}$ & $\begin{array}{l}\text { Feeling pretty good about } \\
\text { Canada right now. } \\
\text { \#Nishiyuu } \\
\text { pic.twitter.com/4ttBuzGenf }\end{array}$ & $\begin{array}{l}2013- \\
03-25 \\
11: 50\end{array}$ & 38 & 20 & 0 & Photo & j & 40 \\
\hline $\begin{array}{l}\text { Nishiyuu } \\
@ \text { Nishiiyuu }\end{array}$ & $\begin{array}{l}\text { Now arriving on Victoria } \\
\text { Island... We can't thank you } \\
\text { enough for your support! } \\
\text { \#Nishiyuu \#March25 } \\
\text { \#Nishiyuuarrivalday } \\
\text { \#Unity \#Idlnomore }\end{array}$ & $\begin{array}{r}2013- \\
03-25 \\
8: 32\end{array}$ & 99 & 26 & 9 & Post & no & 106 \\
\hline
\end{tabular}




\begin{tabular}{|c|c|c|c|c|c|c|c|c|}
\hline $\begin{array}{l}\text { Elizabeth May } \\
\text { MP Verified } \\
\text { account } \\
\text { @ElizabethMay }\end{array}$ & $\begin{array}{l}\text { Huge rally! Thousands } \\
\text { greet \#Nishiyuu walkers on } \\
\text { Parliament Hill! And they } \\
\text { are still pouring in. } \\
\text { \#cdnpoli \#gpc } \\
\text { pic.twitter.com/U8YL7xr21 } \\
9\end{array}$ & $\begin{array}{l}2013- \\
03-25 \\
11: 16\end{array}$ & 53 & 10 & 3 & Photo & $p$ & 55 \\
\hline $\begin{array}{l}\text { Kristy Kirkup } \\
\text { @kkirkup }\end{array}$ & $\begin{array}{l}\text { When young Cree walkers } \\
\text { arrived on Parliament Hill, } \\
\text { they broke down and } \\
\text { started to weep. } 1600 \mathrm{~km} \\
\text { on foot. \#nishiyuu } \\
\text { \#idlenomore \#cdnpoli }\end{array}$ & $\begin{array}{l}2013- \\
03-25 \\
12: 43\end{array}$ & 50 & 4 & 2 & Post & j & 49 \\
\hline $\begin{array}{l}\text { Krystalline Kraus } \\
\text { @krystalline_k }\end{array}$ & $\begin{array}{l}\text { Sign at Ottawa rally, "Pray } \\
\text { for the pandas, they are } \\
\text { stuck with Harper today!" } \\
\text { \#idlenomore \#Nishiyuu } \\
\text { @rabbleca }\end{array}$ & $\begin{array}{r}2013- \\
03-25 \\
9: 43\end{array}$ & 42 & 5 & 1 & Post & j & 43 \\
\hline $\begin{array}{l}\text { Andrew Foote } \\
\text { @amkfoote }\end{array}$ & $\begin{array}{l}\text { Can't tell in a pic but this } \\
\text { rally-ending round dance } \\
\text { stretches all around the } \\
\text { Parliament lawn. \#cbcott } \\
\text { \#Nishiyuu } \\
\text { pic.twitter.com/xyy5GSvuh } \\
\mathrm{t}\end{array}$ & $\begin{array}{l}2013- \\
03-25 \\
13: 52\end{array}$ & 47 & 7 & 3 & Photo & j & 42 \\
\hline $\begin{array}{l}\text { CTV Toronto } \\
\text { @CTVToronto }\end{array}$ & $\begin{array}{l}\text { MT @CTVNews Cheers } \\
\text { greet young Cree walkers } \\
\text { after 1,600-kilometre trek } \\
\text { to Ottawa } \\
\text { http://ow.ly/jp3XJ } \\
\text { \#IdleNoMore \#Nishiyuu }\end{array}$ & $\begin{array}{l}2013- \\
03-25 \\
13: 21\end{array}$ & 18 & 1 & 0 & $\begin{array}{l}\text { Embedded } \\
\text { News } \\
\text { Article/ } \\
\text { Online } \\
\text { Video }\end{array}$ & j & 54 \\
\hline $\begin{array}{l}\text { Hayden King } \\
@ \text { Hayden_King }\end{array}$ & $\begin{array}{l}\text { Amazing to hear the } \\
\text { \#Nishiyuu, one after } \\
\text { another, thank their } \\
\text { grandmothers and } \\
\text { grandfathers for watching } \\
\text { out for them on the walk. }\end{array}$ & $\begin{array}{l}2013- \\
03-25 \\
11: 49\end{array}$ & 35 & 9 & 1 & Post & no & 33 \\
\hline $\begin{array}{l}\text { Andrew Cash } \\
\text { @Cash4TO }\end{array}$ & $\begin{array}{l}\text { \#Nishiyuu walkers arriving } \\
\text { on Parliament Hil now } \\
\text { \#IdleNoMore } \\
\text { pic.twitter.com/H2Q91Qjw } \\
\text { IA }\end{array}$ & $\begin{array}{l}2013- \\
03-25 \\
11: 08\end{array}$ & 25 & 7 & 0 & Photo & $p$ & 24 \\
\hline $\begin{array}{l}\text { leanne simpson } \\
\text { @betasamosak } \\
\text { e }\end{array}$ & $\begin{array}{l}\text { \#Nishiyuu walkers. the first } \\
\text { glimpse of what happens } \\
\text { when you can finally raise } \\
\text { your kids with love, } \\
\text { language, land \& culture. }\end{array}$ & $\begin{array}{r}2013- \\
03-26 \\
7: 58\end{array}$ & 43 & 16 & 1 & Post & j & 44 \\
\hline
\end{tabular}




\begin{tabular}{|c|c|c|c|c|c|c|c|c|}
\hline $\begin{array}{l}\text { Janice Makokis } \\
\text { @bearclannatio } \\
\text { n }\end{array}$ & $\begin{array}{l}\text { \#ldleNoMore woke up a } \\
\text { nation and now \#Nishiyuu } \\
\text { youth inspire a nation. } \\
\text { \#SolidaritySpring off to a } \\
\text { good start in bringing } \\
\text { message of UNITY. }\end{array}$ & $\begin{array}{l}2013- \\
03-25 \\
19: 27\end{array}$ & 35 & 3 & 1 & Post & no & 36 \\
\hline $\begin{array}{l}\text { Idle No More } \\
\text { @Idlenomoreye } \\
\text { g }\end{array}$ & $\begin{array}{l}\text { Harper's welcoming pandas } \\
\text { in Toronto today to avoid } \\
\text { facing the \#nishiyuu } \\
\text { arriving in Ottawa. Respect } \\
\text { fail. Aga... } \\
\text { http://bit.ly/Zl64m1 }\end{array}$ & $\begin{array}{r}2013- \\
03-25 \\
7: 45\end{array}$ & 33 & 1 & 0 & $\begin{array}{l}\text { Post, } \\
\text { hyperlink } \\
\text { (to Twitter } \\
\text { post) }\end{array}$ & no & 33 \\
\hline UBCIC @UBCIC & $\begin{array}{l}\text { RT @eli_d_m: Here is an } \\
\text { update from the } \\
\text { Whapmagoostui youth } \\
\text { walkers: \#nishiyuu } \\
\text { \#idlenomore } \\
\text { pic.twitter.com/0mFLfPQt }\end{array}$ & $\begin{array}{l}2013- \\
01-18 \\
21: 13\end{array}$ & 30 & 7 & 0 & $\begin{array}{l}\text { Photo (of } \\
\text { Facebook } \\
\text { Post) }\end{array}$ & $p$ & 28 \\
\hline $\begin{array}{l}\text { Mad Cow } \\
\text { Toons } \\
\text { @madcowtoons }\end{array}$ & $\begin{array}{l}\text { Mad Cow spends day } \\
\text { kissing Pandas } \\
\text { http://buff.ly/11EGeJS } \\
\text { ignores \#Nishiyuu Youth } \\
\text { who walked } 1600 \mathrm{~K} \text { to see } \\
\text { him! \#idlenomore \#INM } \\
\text { \#canpoli }\end{array}$ & $\begin{array}{l}2013- \\
03-25 \\
17: 13\end{array}$ & 45 & 7 & 0 & $\begin{array}{l}\text { Post, } \\
\text { Hyperlink } \\
\text { to Photo }\end{array}$ & j & 40 \\
\hline $\begin{array}{l}\text { Derrick O'Keefe } \\
\text { @derrickokeefe }\end{array}$ & $\begin{array}{l}\text { Great to see \#Nishiyuu get } \\
\text { global coverage - here's } \\
\text { BBC } \\
\text { http://www.bbc.co.uk/new } \\
\text { s/world-us-canada- } \\
21934058 \text {... \#ldleNoMore } \\
\text { \#cdnpoli }\end{array}$ & $\begin{array}{l}2013- \\
03-25 \\
23: 03\end{array}$ & 28 & 3 & 1 & $\begin{array}{l}\text { Hyperlink } \\
\text { to News } \\
\text { Article }\end{array}$ & j & 29 \\
\hline
\end{tabular}

Figure 28: Table showing top 36 \#Nishiyuu tweets by volume ${ }^{426}$. Table shows User Name, Tweet text-only Content, Date and Time, and Volumetric data such as number of Retweets, Replies, Favourites, and Associated Tweets.

${ }^{426}$ Using Topsy.com (Accessed: November 11 2013) 


\section{C.3 Volumetric Charts}

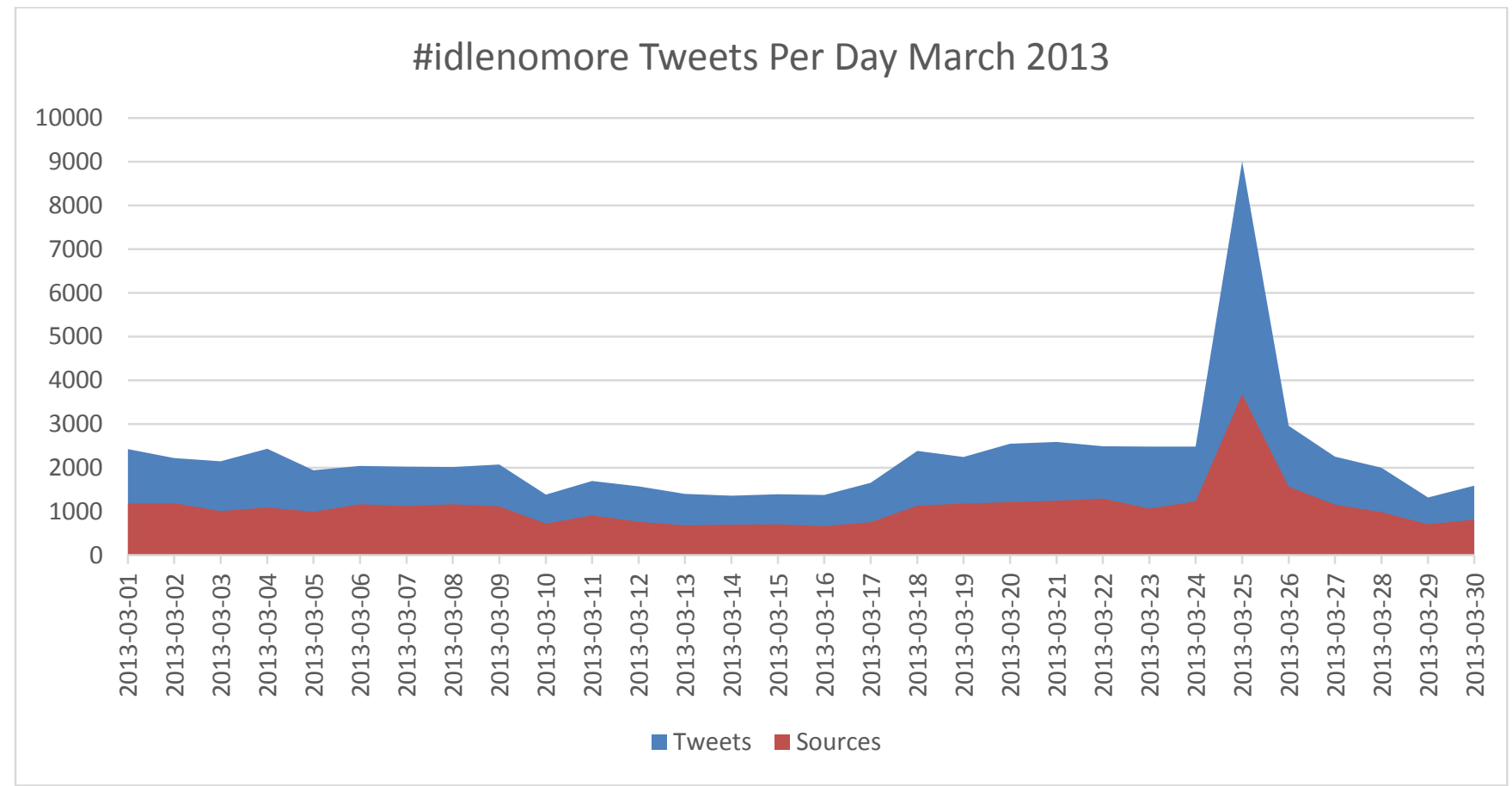

Figure 29: \#idlenomore tweet volume for March $1^{\text {st }}$ to 30 th $2013^{427}$

\section{Facebook Group Posts March 2013}

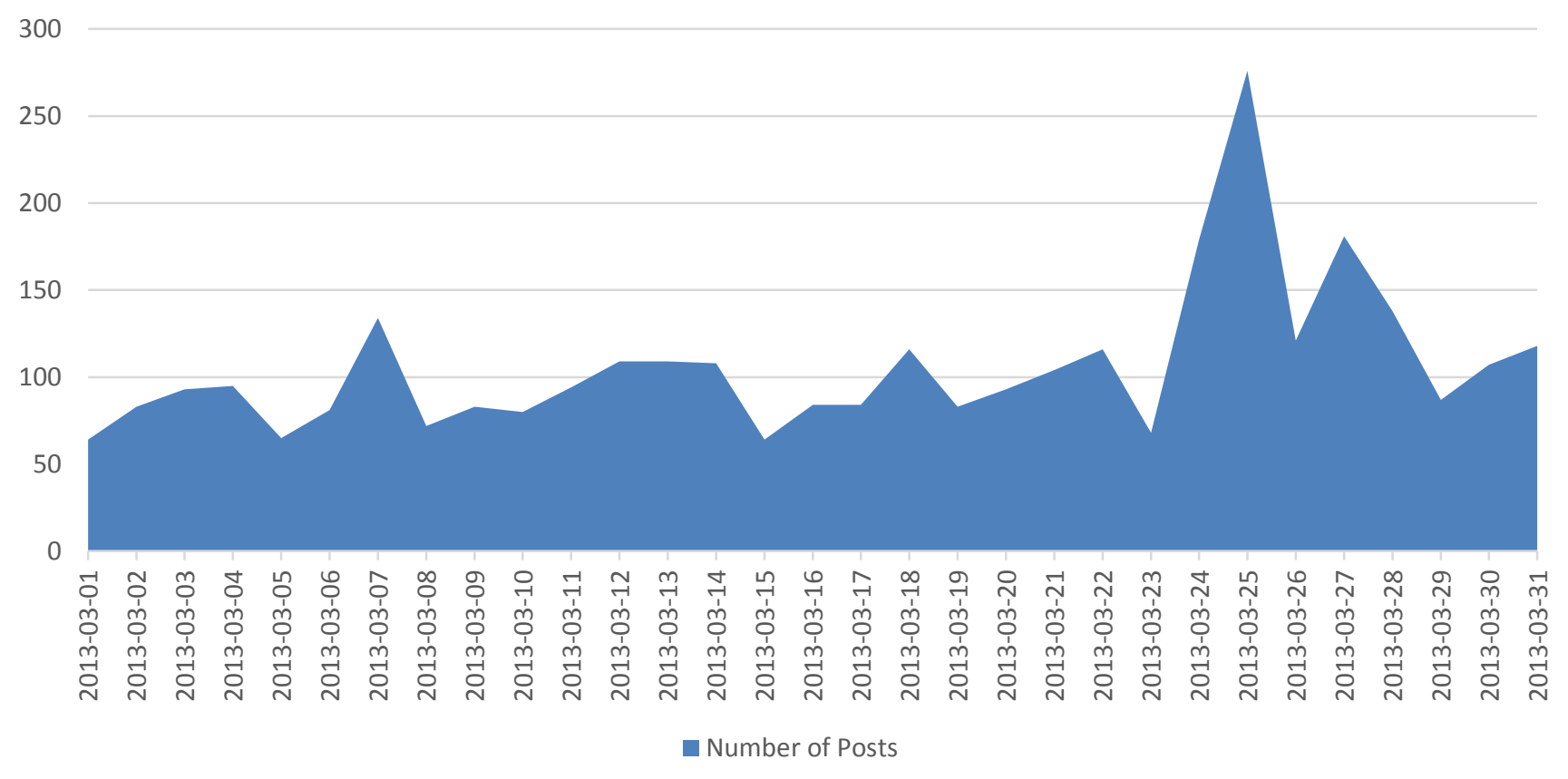

${ }^{427}$ Created using data from Mark Blevis: Digital Public Affairs retrieved from markblevis.com; see Blevis (March 3, 2013); Blevis (March 10, 2013); Blevis (March 17, 2013); Blevis (March 24, 2013); and Blevis (March 31, 2013); 


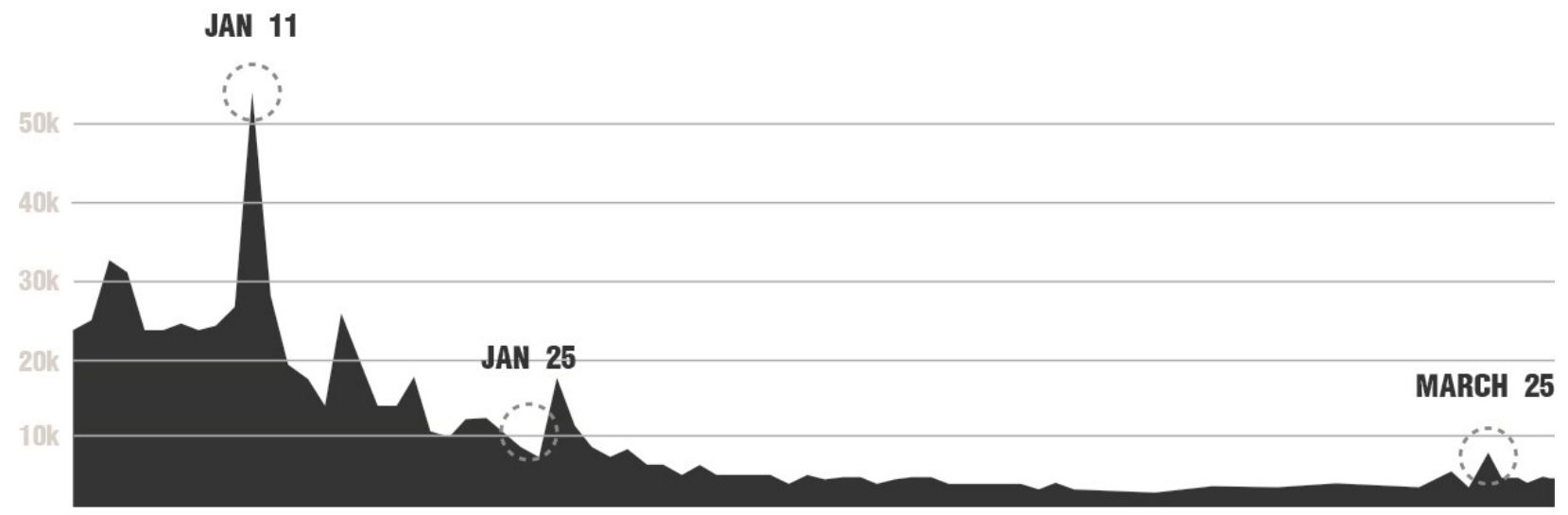

Figure 31: "Tweet volume of \#idlenomore hashtag adapted from Topsy. (Topsy, 2013)" 429 Shows longitudinal data for \#idlenomore preceding the March 25th arrival of the Nishiyuu walkers and covering the full timespan that the youths were underway (January 16 $6^{\text {th }}$ March 25th 2013).

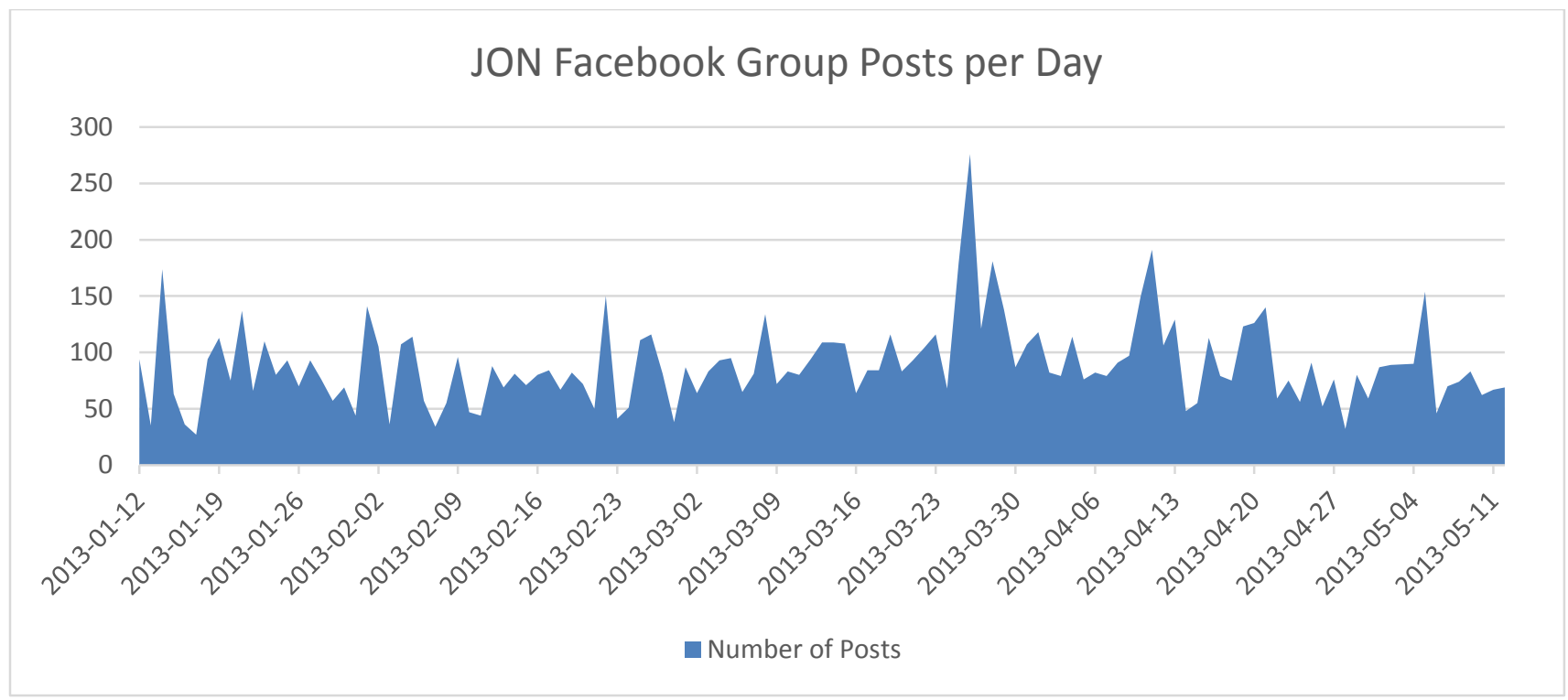

Figure 32: Volumetric Timeline representing the number of posts per day to the JON Facebook group page. Shows longitudinal data for posting activity on the Facebook group page devoted to the JON.

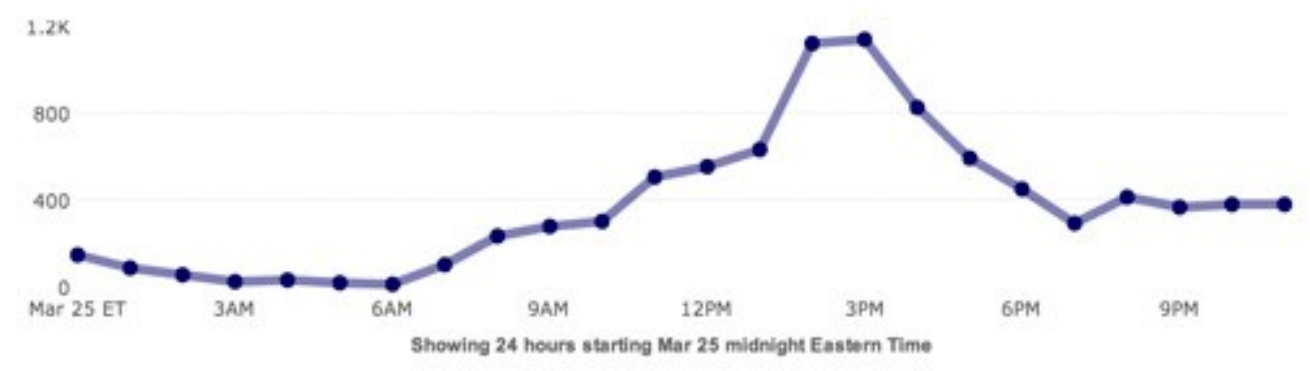

${ }^{428}$ Data collected using Facebook API. Number of independent posts exclusive other activity including comments, likes (of both posts and comments), shares external to the group page, and amendments to original posts ${ }^{429}$ Gregoriev 2013; Chart shows date range from January $1^{\text {st }}$ to March $30^{\text {th }} 2013$ 
Figure 33: Data showing \#idlenomore volumes on arrival of Nishiyuu walkers (March $\left.25^{\text {th }} 2013\right)^{430}$

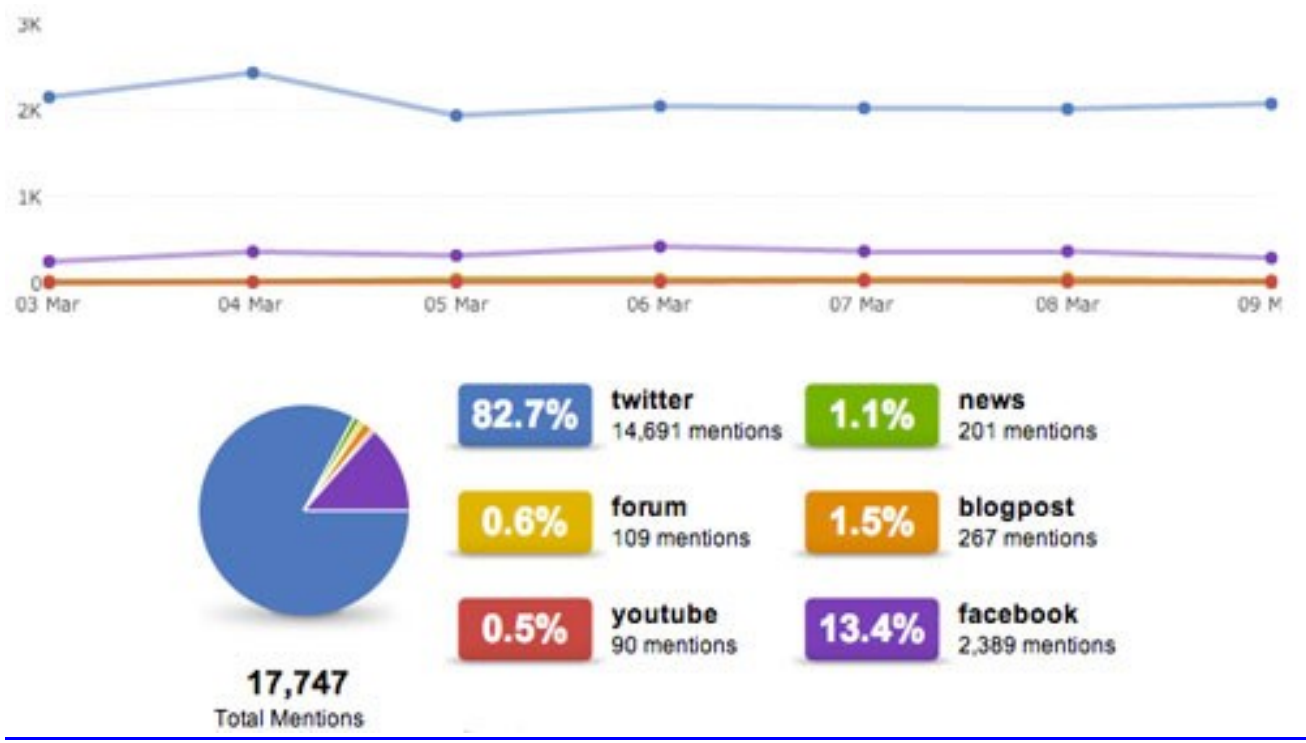

1. Total Mentions

Figure 34: Data showing idlenomore traffic for the Week of 3-9 March 2013

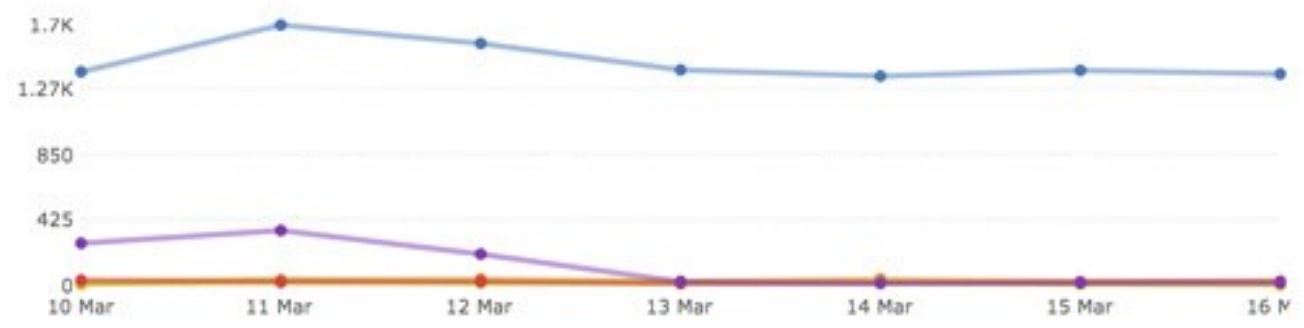

2.
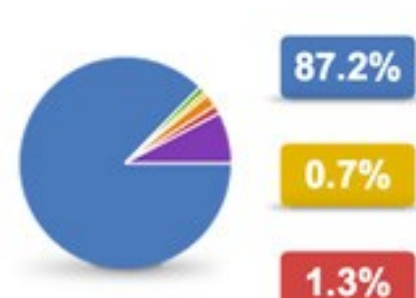

twitter

10,200 mentions

\section{$1.0 \%$}

news

\section{$0.7 \%$ forum}

87 mentions

$1.9 \%$

blogpost

\section{$1.3 \%$ youtube}

\section{$7.7 \%$}

facebook

11,688

152 mentions

Total Mentions

Figure 35: Data showing idlenomore traffic for the Week of 10-17 March $2013^{431}$

${ }^{430}$ Blevis, Mark (March 26, 2013)

${ }^{431}$ Blevis, Mark (March 17, 2013) 


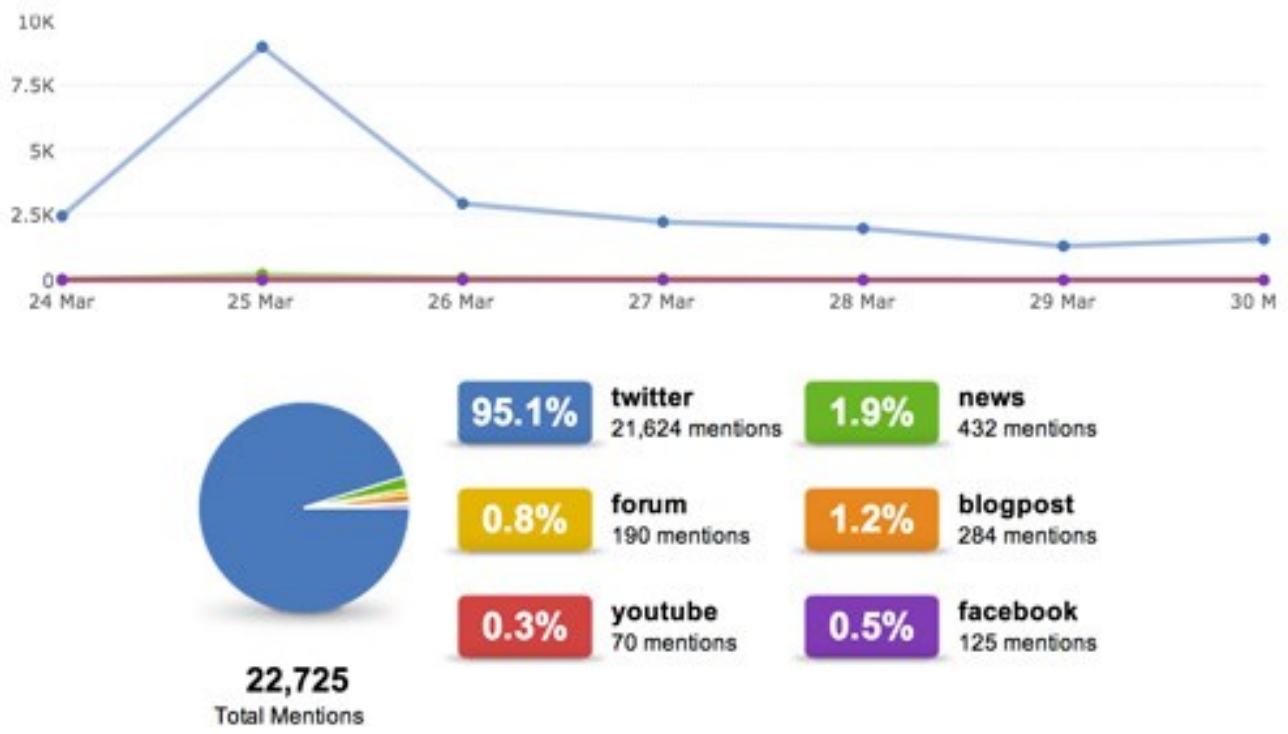

Figure 36: Data showing idlenomore traffic for the Week of 24-30 March $2013^{432}$

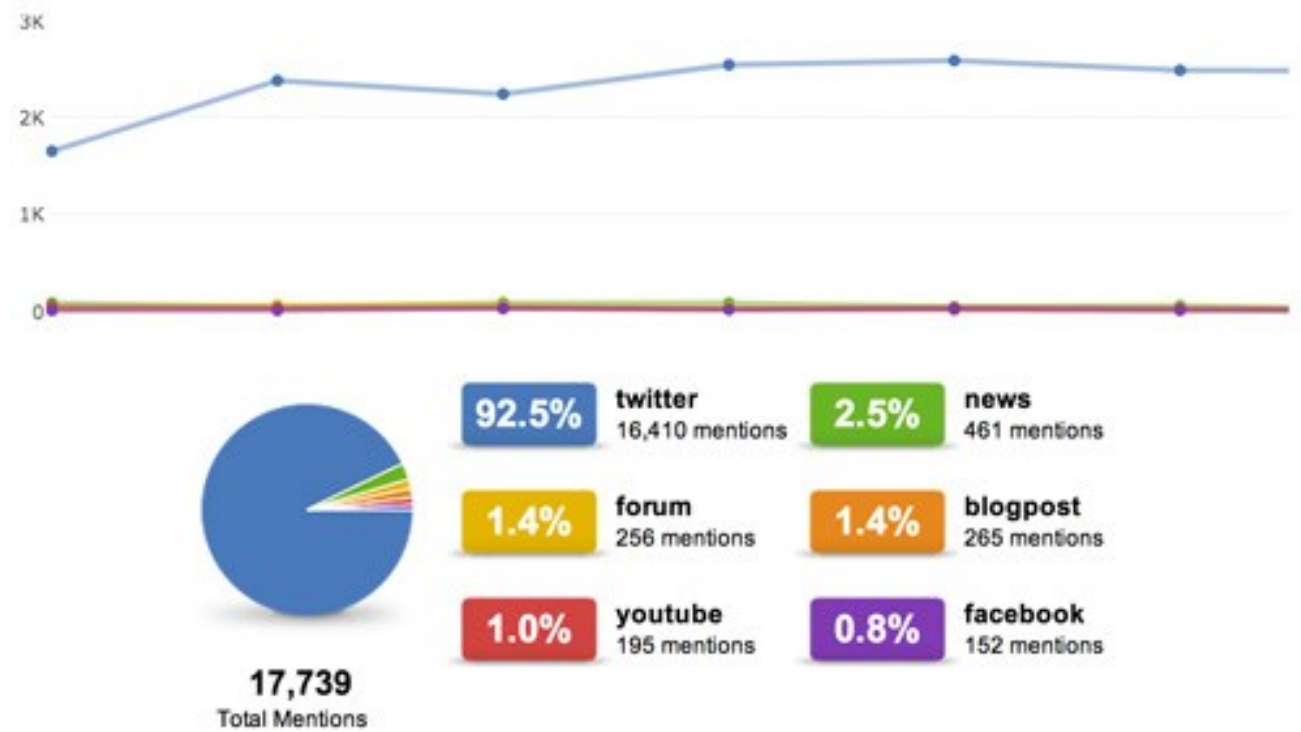

Figure 37: Data showing idlenomore traffic for the Week of 24-30 March $2013^{433}$

432 Blevis, Mark (March 31, 2013)

${ }^{433}$ Blevis, Mark (March 31, 2013) 


\section{References (Figures)}

AANDC (March 2013) "Aboriginal Connectivity Profiles of Quebec as of March 2013" AANDC.ca Last accessed: February 232014 http://www.aadncaandc.gc.ca/eng/1352223782819/1353504825398?p=qc

Ahki (Last Modified: March 28 ${ }^{\text {th }}$ 2014) “The Journey of Nishiyuu" Last accessed April $2^{\text {nd }} 2013$ from: http://www.ahki.ca/journey-of-nishiyuu.php Last accessed: Feb 15 2014; permanent URL: http://www.ahki.ca/resources/Whapmagoostui\%20Youth\%20walkers.jpg

Barham, Andy (May 14 ${ }^{\text {th }}$ 2013) "Sadly, the mainstream media paid even less attention to this long trek than they did to yours [...]" Facebook - Journey of Nishiyuu [Facebook Post ] Lasted accessed April $3^{\text {rd }}$ 2013, Permanent URL:

https://www.facebook.com/groups/nishiyuu/permalink/412791345494913

Bearskin, Mina Elizabeth (March 11 ${ }^{\text {th }}$ 2013) "Lessons Learned in Life Photo" [Facebook Post] Last accessed: March 42014 From: https://www.facebook.com/groups/nishiyuu/permalink/383162085124506/

Blevis, Mark (March 3, 2013) "Summary of IdleNoMore traffic for Feb 24- Mar 2" Mark Blevis: Digital Public Affairs [Blog Post] retrieved April 2 ${ }^{\text {nd }}, 2014$, from http://markblevis.com/summary-of-idlenomore-traffic-for-feb-24-mar-2/

Blevis, Mark (March 10, 2013) "Summary of IdleNoMore traffic for Mar 3-9" Mark Blevis: Digital Public Affairs [Blog Post] retrieved March 31, 2014, URL: http://markblevis.com/summary-of-idlenomore-traffic-for-mar-3-9/

Blevis, Mark (March 17, 2013) "Summary of IdleNoMore traffic for Mar 10-16" Mark Blevis: Digital Public Affairs [Blog Post] retrieved March 31, 2014, URL: http://markblevis.com/summary-of-idlenomore-traffic-for-mar-10-16/

Blevis, Mark (March 24, 2013) "Summary of IdleNoMore traffic for Mar 17-23" Mark Blevis: Digital Public Affairs [Blog Post] retrieved March 31, 2014, URL: http://markblevis.com/summary-of-idlenomore-traffic-for-mar-17-23/

Blevis, Mark (March 26, 2013) "The Journey of Nishiyuu re-energizes the IdleNoMore movement" Mark Blevis: Digital Public Affairs [Blog Post] retrieved March 31, 2014, URL: http://markblevis.com/the-journey-of-nishiyuu-re-energizes-the-idlenomoremovement/ 
Blevis, Mark (March 31, 2013) "Summary of IdleNoMore traffic for Mar 24-30" Mark Blevis: Digital Public Affairs [Blog Post] retrieved March 31, 2014, URL: http://markblevis.com/summary-of-idlenomore-traffic-for-mar-24-30/

'derrick' (March 17 2013) "Under the mainstream media's radar, an historic journey..." Rabble.ca - Babble [Forum Post] Last Accessed: March 30 2013, URL: http://rabble.ca/babble/aboriginal-issues-and-culture/historic-journey-indigenous-youthset-reach-parliament-hill-mar

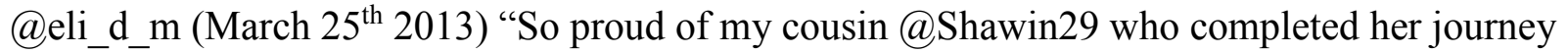
today!! \#Nishiyuu \#IdleNoMore pic.twitter.com/fzmtx6iLKI" [Tweet] Last accessed: Feb 152014 from: https://twitter.com/eli d m/status/316388613363466240

Galloway, Gloria (March 25 $5^{\text {th }}$ 2013) "Nishiyuu a Movement of Cree Youth Who Voted with Their Feet" [Comments Section] The Globe and Mail Last accessed: March 30 2014, URL: http://www.theglobeandmail.com/news/politics/nishiyuu-a-movement-of-creeyouth-who-voted-with-their-feet/article10327993/comments/

Gregoriev, Michael 2013 "Leveraging Collective Intellegence in Participatory Design Research Using Information and Communications Technology" Master's of Design Thesis Carleton University

Gups-Y-Bees Lepled (March 9, 2013) "The Journey Of Nishiyuu Getting Closer" Gups-Y-Bees The Answer Is 42 [Blog Post] Image attributed to: Robert Savoie Last Accessed April $3^{\text {rd }}$ 2013 URL: http://thehairybuddha.files.wordpress.com/2013/03/gc.jpg?w=1176

Howard, Darrin (March 31 ${ }^{\text {st }}$ 2013) "Since Manstream Media didn't cover it [...] we did..."

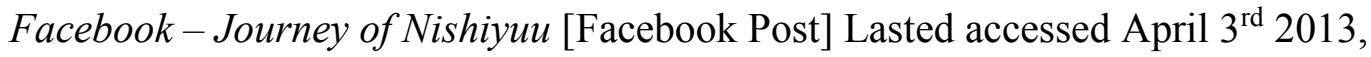
Permanent URL: https://www.facebook.com/groups/nishiyuu/permalink/393260227448025

Jay Jay House (March 28 2013) "I took this picture when they were walking towards Chelsea, I have no idea who..." Facebook - Journey of Nishiyuu [Facebook Post] Last accessed: March 252014 URL:

https://www.facebook.com/photo.php?fbid=574525369234001\&set=gm.39186983092039 $\underline{8 \text { \&type }}=1 \&$ theater

Kawapit, David (March 24, 2013) "CBC interview with David Kawapit live at 3:00 PM Eastern Time”. Facebook - Journey of Nishiyuu [Facebook Post] Last accessed:March 302014 
Permanent URL:

https://www.facebook.com/groups/nishiyuu/permalink/390028701104511

"Logo" (January 6 $6^{\text {th }} 2013$ ) [Image] Last accessed: April $2^{\text {nd }} 2014$ URL:

http://nishiyuujourney.ca/wp-content/uploads/2013/02/nishiyuu_logo_final_352.png

Liz_Wilson_2 (March 25 2013) "The real travesty is the amount of time the media is devoting to the Pandas..." Huffington Post [Conversation Thread] Last accessed: March 292014 URL: http://www.huffingtonpost.ca/2013/03/25/nishiyuu-journey-ends-ottawa-harperpandas n 2950643.html\#conversation-2950643-239806344

'Meme' (Modified March 27 $7^{\text {th }}$ 2013) Google image search “\#nishiyuu” URL: https://pbs.twimg.com/media/BGZz7ooCQAAntJg.jpg Last accessed: April 2nd 2013

Mukash, Sage (January 23, 2013) "The Journey of Nishiyuu - A Very Important Piece of Native Culture (as well as other cultures) Is Humour" The Watchman [Blog Post] Last accessed: April $3^{\text {rd }} 2014$ URL: http://3.bp.blogspot.com/t2ssjzE2bVQ/URClsfazklI/AAAAAAAANr4/0JS9Jsyw8YQ/s1600/ journey_itinerary2_robert_savoie.jpg

Pearson, Matthew (March 25 ${ }^{\text {th }}$ 2013) "Nishiyuu Walkers Ranks Swollen from Seven to Several Hundred" Ottawa Citizen [Comments Section] last accessed April $2^{\text {nd }} 2014$, URL: http://www.ottawacitizen.com/Nishiyuu+Walkers+reach+Chelsea+their+ranks+swollen+fr om+seven+several+hundred/8145736/story.html

Powless, Ben (March 26, 2013) "Fresh picks from the journey of \#Nishiyuu welcoming Ottawa at flicr.com/photos/powless... \#idlenomore" [Tweet] Last accessed: Feb 152014 URL: https://twitter.com/redman0380/status/316442839078825984

Srgtsnakeeyes11 (March 25 2013) "Harper's personal Agenda has always come first..." Huffington Post [Conversation Thread] Last accessed: March 302014 URL: http://www.huffingtonpost.ca/2013/03/25/nishiyuu-journey-ends-ottawa-harperpandas n 2950643.html\#conversation-2950643-239808503

Strapagie, Lauren (March 25 2013) "Nishiyuu Journey By Cree Youth Ends As Harper Greets Pandas (TWEETS, VIDEO)" Huffington Post Canada [Comments Section] [Posted: 03/25/2013 4:34 pm EDT ; Updated: 03/26/2013 6:24 am EDT] Last accessed: March 30 2014 URL: http://www.huffingtonpost.ca/2013/03/25/nishiyuu-journey-ends-ottawaharper-pandas_n_2950643.html 
Whiskeychan, Jimmy Tim (February 24, 2013) “On their way to km 98 February 24, 2013 in Waskaganish" [Facebook Post] Last accessed: March 302014 from: https://www.facebook.com/groups/nishiyuu/permalink/412791345494913/?stream_ref=21

Yahoo! Canada News (March 26, 2013) "Did Stephen Harper slight the Nishiyuu Walkers?" in Pulse of Canada (Comments section) Last Accessed April $4^{\text {th }} 2013$ URL: https://ca.news.yahoo.com/blogs/pulseofcanada/did-stephen-harper-slight-nishiyuuwalkers-145121229.html

@ FatehBorn (March 26 $6^{\text {th }} 2013$ ) "Beautiful summary of \#Nishiyuu walkers $1600 \mathrm{~km}$ journey mostly on snowshoes.\#idlenomore http://youtu.be/jqqNVgfOQH0" [Tweet] Last Accessed April $2^{\text {nd }} 2014$ URL: https://twitter.com/FatehBorn/status/316602889168621568,

@ Idlenomore4 (March 26 $6^{\text {th }}$ 2013) “\#Nishiyuu: A movement of Cree youth who voted with their feet http://www.theglobeandmail.com/news/politics/nishiyuu-a-movement-of-cree-youthwho-voted-with-their-feet/article10327993/ ..." [Tweet] Last Accessed April $2^{\text {nd }} 2014$, URL: https://twitter.com/FatehBorn/status/316602889168621568

@ jianghomeshi (March $25^{\text {th }}$ 2013) "The \#Nishiyuu walkers are a true inspiration. And deserve more attention than visiting pandas. - my opening essay tmrw morn. \#Q" [Tweet] Last accessed: March 4 2014, URL:

https://twitter.com/jianghomeshi/statuses/316312362632216576 


\section{Bibliography}

Atton, C. (2003). Reshaping Social Movement Media for a New Millennium. Social Movement Studies, 2(1), 3-15. doi:10.1080/1474283032000062530

Bailenson, J. N., Yee, N., Blascovich, J., \& Guadagno, R. E. (2008). Transformed social interaction in mediated interpersonal communication. (E. Konijn, M. Tanis, S. Utz, \& A. Linden, Eds.)Mediated interpersonal communication, 9(3), 77-99. Retrieved from

http://books.google.com/books?hl=en\&amp;lr=\&amp;id=9x_7AKBgAfkC\&amp;oi=fnd\&amp;pg= PA77\&amp;dq=Transformed +social+interaction+in+mediated + interpersonal + communication\&am p;ots=JmcxzlwYNR\&amp;sig=S7Zy-eGBBNoddfcldCDjW0R6H1I

Barthes, R., \& Miller, R. (1975). The Pleasure of the Text. Farrar, Straus and Giroux. Retrieved from http://books.google.ca/books?id=VMazbXKwPGoC

Batinic, B., Reips, U., \& Bosnjak, M. (2002). Online social sciences. Retrieved from http://psycnet.apa.org/psycinfo/2003-88135-000

Baudrillard, J., \& Lotringer, S. (1988). The ecstasy of communication. Autonomedia. Retrieved from http://books.google.ca/books?id=CVsQAQAAIAAJ

BBC.com (March 31, 2014) "Turkey PM Erdogan claims election victory” Last Accessed March 312014 URL: http://www.bbc.com/news/world-europe-26807067

Benjamin, W. (1935). The Work of Art in the Age of Mechanical Reproduction.

Bernstein, M., Bakshy, E., Burke, M., \& Karrer, B. (2013). "Quantifying the Invisible Audience in Social Networks". Proceedings of the SIGCHI .... Retrieved from http://dl.acm.org/citation.cfm?id=2470658

Biscuitwala, K., Bult, W., \& Lécuyer, M. (2013). Weaving a safe web of news. ... on World Wide Web ..., 849-852. Retrieved from http://dl.acm.org/citation.cfm?id=2488063

Bolter, J D. (1991). Writing Space: Computers, Hypertext, and the History of Writing. Lawrence Erlbaum Associates: Hillsdale, NJ

Bolter, J. David, \& Grusin, R. a. (1996). Remediation. Configurations, 4(3), 311-358. doi:10.1353/con.1996.0018

Bush, V. (1945). As We May Think, in The Atlantic Monthly. July 1945. Reprinted in ACM Interactions 3(2), March 1996, 35-46.

Byron, K. (2008). Carrying too Heavy a Load? The Communication and Miscommunication of Emotion by Email. Academy of Management Review, 33(2), 309-327. doi:10.5465/AMR.2008.31193163 
CBC.ca (March 25 2013) “Cree Walkers meet Minister at end of Idle No More Trek” on Yahoo! Canada News Last Accessed April 4, 2014 URL: https://ca.news.yahoo.com/journey-nishiyuu-end-ottawamorning-104314582.html

CBC.ca (September 16, 2013) “Terms of Use” CBC Policies Last Accessed: March 14, 2014 URL: http://www.cbc.ca/aboutcbc/discover/termsofuse.html

CerAmony Band (July 24 2013) “Tribute to Isaac Kawapit 'The White Wizard' of the Journey of Nishiyuu” YouTube.com [YouTube Slideshow/Video] Last Accessed: April 102013 URL: http://www.youtube.com/watch?v=SvABF07E4Q0

Chandler, D., \& Munday, R. (2011). "Communication." In A Dictionary of Media and Communication. : Oxford University Press. Retrieved 22 Mar. 2014, from http://www.oxfordreference.com.proxy.library.carleton.ca/view/10.1093/acref/9780199568758.001 $.0001 /$ acref-9780199568758-e-0413.

Chandler, D., \& Munday, R. (2011). “Affordances.” In A Dictionary of Media and Communication. : Oxford University Press.

Chandler, D., \& Munday, R. (2011). “Computer-Mediated Communication.” In A Dictionary of Media and Communication. : Oxford University Press.

Chandler, D., \& Munday, R. (2011). “Online Communication.” In A Dictionary of Media and Communication. : Oxford University Press.

Cheshin, A., Rafaeli, A., \& Bos, N. (2011). Anger and happiness in virtual teams: Emotional influences of text and behavior on others' affect in the absence of non-verbal cues. Organizational Behavior and Human Decision Processes, 116(1), 2-16. doi:10.1016/j.obhdp.2011.06.002

Choudhury, M. De, \& John, A. (2009). What Makes Conversations Interesting ? Themes, Participants and Consequences of Conversations in Online Social Media, 331-340.

Choudhury, M. De, Sundaram, H., John, A., \& Seligmann, D. (2010). Analyzing the dynamics of communication in online social networks. Retrieved from http://link.springer.com/chapter/10.1007/978-1-4419-7142-5_4

Conklin, J. (1987). Hypertext: An Introduction and SurvevJ, (September). Retrieved from http://www.ics.uci.edu/ andre/informatics223s2007/conklin.pdf

CTV.ca News Staff (April 5 2011) "Harper criticized for 'unfriending' teen from rally" Published Tuesday, April 5, 2011 9:09PM EDT Last Accessed Mar. 222014 URL: http://www.ctvnews.ca/harper-criticized-for-unfriending-teen-from-rally1.627752\#ixzz2wkojmuIE 
De Landa, M. (1997). A thousand years of nonlinear history. Zone Books. Retrieved from http://books.google.ca/books?id=4GNQAAAAMAAJ

DeLuca, K. M., Lawson, S., \& Sun, Y. (2012). Occupy Wall Street on the Public Screens of Social Media: The Many Framings of the Birth of a Protest Movement. Communication, Culture \& Critique, 5(4), 483-509. doi:10.1111/j.1753-9137.2012.01141.x

Donath, J., \& Viégas, F. (2002). The chat circles series. Proceedings of the conference on Designing interactive systems processes, practices, methods, and techniques - DIS '02, 359-369. doi:10.1145/778757.778764

Dourish, P., \& Bell, G. (2011). Divining a digital future. Mit Press. Retrieved from http://books.google.ca/books?id=Skx-sEXG1_QC

Drucker, J. (n.d.). Humanistic Theory and Digital Scholarship.

Eco, U., \& Collini, S. (1992). Interpretation and Overinterpretation. Cambridge University Press. Retrieved from http://books.google.ca/books?id=wbhROmD3guQC

Edwards, Charlie (2012) The Digital Humanities and Its Users" in Debates in the Digital Humanities edited by Matthew K. Gold University of Minnesota press: Minneapolis, MN. P 213-232

Ess, Charles (2002)"Cultures in Collision: Philosophical Lessons from Computer-Mediated Communication" in Cyber Philosophy: the intersection of computing and philosophy ed James h. Moor and Terrell Ward Bynum; Blackwell Publishiing: Malden, MA

Facebook (n.d) "News Feeds" Facebook - Help Last accessed March 152014 URL: https:/www.facebook.com/help/166738576721085

Fernback, J. (2003). Legends on the net: an examination of computer-mediated communication as a locus of oral culture. New Media \& Society, 5(1), 29-45. doi:10.1177/1461444803005001902

Fragoso, S., Rebs, R., \& Barth, D. (2012). Interface affordances and social practices in online communication systems. Proceedings of the International .... Retrieved from http://dl.acm.org/citation.cfm?id=2254569

Freedman, D. (2002). A “Technological Idiot”? Raymond Williams and Communications Technology. Information, Communication \& Society, 5(3), 425-442. doi:10.1080/13691180210159346

Gaines, B. R. and Shaw, M. L. G (1984) The Art of Computer Conversation: A New Medium for Communication Prentice/Hall: Toronto, ON 
Gill, A. J., Gergle, D., French, R. M., \& Oberlander, J. (2008). Emotion rating from short blog texts. Proceeding of the twentysixth annual CHI conference on Human factors in computing systems CHI 08, (March 1946), 1121. doi:10.1145/1357054.1357229

Graham, G., \& Greenhill, A. (2013). Exploring Interaction: Print and Online News Media Synergies. Internet Research, 23(1), 89-108. doi:10.1108/10662241311295791

Hancock, J. T., Gee, K., Ciaccio, K., \& Lin, J. M. (2008). I' m Sad You' re Sad : Emotional Contagion in CMC, 295-298.

Hansen, D., Shneiderman, B., \& Smith, M. A. (2011). Chapter I: Getting Started with Analysing Social Media Networks. In Analyzing Social Media Networks with NodeXL: Insights from a Connected World (pp. 3-31). Burlington, MA: Elsevier Inc.

Hansen, Derek \& Shneiderman, Ben \& Smith, Marc. (2011). "Chapter 7 - Clustering and Grouping” Analyzing Social Media Networks with NodeXL: Insights from a Connected World. [Books24x7 version] Available from http://common.books24x7.com.proxy.library.carleton.ca/toc. aspx?bookid=40146.

Hayles, K. N. (2012). How We Read : Close, Hyper, Machine. In How We think: Digital Media and Contemporary Technogenesis (pp. 55-79). University of Chicago Press. doi:10.1632/ade.150.62

Henderson, S., \& Gilding, M. (2004). "I've Never Clicked this Much with Anyone in My Life': Trust and Hyperpersonal Communication in Online Friendships. New Media \& Society, 6(4), 487-506. doi: $10.1177 / 146144804044331$

Hunt, D., Atkin, D., \& Krishnan, A. (2012). The Influence of Computer-Mediated Communication Apprehension on Motives for Facebook Use. Journal of Broadcasting \& Electronic Media, 56(2), 187-202. doi:10.1080/08838151.2012.678717

Hutchby, Ian (2001) Conversation and technology: From the Telephone to the Internet. Polity Press: Malden, MA

Jubril and Abdulla 2013 "Relevance of Emoticons in Computer-Mediated Communication Contexts: An Overview” Asian Social Science; Vol. 9, No. 4; 2013

Kay, Alan "User Interface: a Personal View" in The Art of Human Computer Interface Design edited by Brenda Laurel (Reading, MA: Addison-Wesley, 1990) 1993

Kaplan, A., \& Haenlein, M. (2010). Users of the world, unite! The challenges and opportunities of Social Media. Business horizons, (53), 59-68. Retrieved from http://www.sciencedirect.com/science/article/pii/S0007681309001232 
Kawapit, David (March 24, 2013) "CBC interview with David Kawapit live at 3:00 PM Eastern Time". Facebook - Journey of Nishiyuu [Facebook Comment Thread] Last accessed: March 302014 Permanent URL: https://www.facebook.com/groups/nishiyuu/permalink/390028701104511

Kramer, A. (2012). The spread of emotion via facebook. Proceedings of the SIGCHI Conference on Human ..., 767-770. Retrieved from http://dl.acm.org/citation.cfm?id=2207787

Landert, D., \& Jucker, A. H. (2011). Private and public in mass media communication: From letters to the editor to online commentaries. Journal of Pragmatics, 43(5), 1422-1434.

doi:10.1016/j.pragma.2010.10.016

Lim, M. (2012). Clicks, Cabs, and Coffee Houses: Social Media and Oppositional Movements in Egypt, 2004-2011. Journal of Communication, 62(2), 231-248. doi:10.1111/j.1460-2466.2012.01628.x

Lim, S., Cha, S. Y., Park, C., Lee, I., \& Kim, J. (2012). Getting closer and experiencing together: Antecedents and consequences of psychological distance in social media-enhanced real-time streaming video. Computers in Human Behavior, 28(4), 1365-1378. doi:10.1016/j.chb.2012.02.022

Little, Jaime (Feb 11, 2014) “Epic Journey of Cree Youth Charted in New Documentary" CBC News Last accessed February 21, 2014, URL: Http://www.cbc.ca/news/aboriginal/epic-journey-of-cree-youthcharted-in-new-documentary-1.2532747

Manovich, L. (1999). Database as symbolic form. ... : The International Journal of Research into New .... Retrieved from $h t t p: / / c o n . s a g e p u b . c o m / c o n t e n t / 5 / 2 / 80$.short

McLuhan, M. (2011). The Gutenberg Galaxy. University of Toronto Press, Scholarly Publishing Division. Retrieved from http://books.google.ca/books?id=7K7rKIWaXXIC

McLuhan, Marshall, 1911-1980. Essential McLuhan. "Chapter 9: Understanding Media": Taylor \& Francis, 2005. 27 October $2012<$ http://lib.myilibrary.com?ID=22131>

McLuhan, M. (1978). Television : Technology and Cultural Form by Raymond Williams Review by : Marshall McLuhan. Technology and Culture, 19(2), 259-261. Retrieved from http://www.jstor.org/stable/3103751 .

Moggridge, B. (2007). Designing Interactions. Cambridge, MA: The MIT Press.

Momoc, A. (2011). New Media and Social Media in the Political Communication, 556-562.

Murthy, D. (2008). Digital Ethnography: An Examination of the Use of New Technologies for Social Research. Sociology, 42(5), 837-855. doi:10.1177/0038038508094565 
Nelson (1973) From: Wardrip-Fruin, Noah and Montfort, Nick "Chapter 21: From Computer Lib / Dream Machines Theodor H. Nelson, 1970-1974" in theNEWMEDIAREADER: The MIT Press: Cambridge and London 2003. P 301-338

Neuman, W. R. (1977). "Television : Technology and Cultural Form . by Raymond Williams Review by : W . Russell Neuman.” American Journal of Sociology, 83(2), 501-503. Retrieved from http://www.jstor.org/stable/2778319

Nishiyuujourney.ca (March 7 2013) "Breaking 30,000 on the Journey of Nishiyuu Group Page!" Nishiyuujourney.ca - Blog Posted [Blog Post] Last Accessed November 142013 URL: http://nishiyuujourney.ca/\#!/?p=637

Nishiyuujourney.ca (n.d) “About” Nishiyuu Journey - About [Webpage] Last Accessed: March 242014 URL: http://nishiyuujourney.ca/\#!/?page_id=10

Nowviskie, Bethany (2012) "Eternal September of the Digital Humanities" Debates in the Digital Humanities ed Matthew K. Gold. University of Minnesota Press: Minneapolis, MN p 243-246

Ogura, K., Matsumoto, Y., Yamauchi, Y., \& Nishimoto, K. (2010). Kairos Chat : A Novel Text-Based Chat System that has Multiple Streams of Time, in Computing Systems 3721-3726. Retrieved from http://dl.acm.org/citation.cfm?id=1754045

Ottereyes (March 25 2013) “Pandas over Natives?!” Patromanottereyes333.blogspot.ca [Blog Post] Last Accessed April 22014 URL: http://patromanottereyes333.blogspot.ca/2013/03/pandas-over$\underline{\text { natives.html }}$

Ottereyes, Parick Roman (March 252013 ) "I wrote a blog post about harper. Please read it and comment your ideas http://patromanottereyes333.blogspot.ca/2013/03/pandas-over-natives.html” FacebookJourney of Nishiyuu [Facebook Post] Last Accessed April 42014 URL:

https:/www.facebook.com/groups/nishiyuu/search/?query $=$ cbc

Oviatt, S., Cohen, A., Miller, A., Hodge, K., \& Mann, A. (2012). The impact of interface affordances on human ideation, problem solving, and inferential reasoning. ACM Transactions on ComputerHuman Interaction, 19(3), 1-30. doi:10.1145/2362364.2362370

Paglia, Camille (2003) "Dispatches from the New Frontier: Writing for the Internet" Communication and Cyberspace: Social Interaction in the Electronic Environment edited by Lance Strate, Ron L. Jacobson, and Stephanie Gibson; Hampton press: Cresskill, NJ 265-276]

Palme, E., Dellarocas, C., Calin, M., \& Sutanto, J. (2012). Attention allocation in information-rich environments: the case of news aggregators. Proceedings of the 14 th .... Retrieved from http://dl.acm.org/citation.cfm?id=2346540

Pearson, Matthew (March 25, 2013) "Nishiyuu Walkers Reached Chelsea Their Ranks Swollen from Seven to Several Hundred" Ottawa Citizen Last Accessed April 6, 2013 URL: 
http://www.ottawacitizen.com/Nishiyuu+Walkers+reach+Chelsea+their+ranks+swollen+from+sev en+several+hundred/8145736/story.html

Pirzadeh, A., \& Pfaff, M. (2012). Expression of emotion in IM. Proceedings of the ACM 2012 conference on ..., 199-202. Retrieved from http://dl.acm.org/citation.cfm?id=2141577

Rabble.ca (n.d) “About” [Webpage] Last accessed: March 312014 from: http://rabble.ca/about/landing

Segerberg, A., \& Bennett, W. L. (2011). Social Media and the Organization of Collective Action: Using Twitter to Explore the Ecologies of Two Climate Change Protests. The Communication Review, 14(3), 197-215. doi:10.1080/10714421.2011.597250

Shirazi, F. (2013). Social media and the social movements in the Middle East and North Africa: A critical discourse analysis. Information Technology \& People, 26(1), 28-49.

doi:10.1108/09593841311307123

Simanowski, Roberto (2013) "Text as Event: Calm Technology and Invisible Information as Subject of Digital Arts" in Throughout: Art and Culture Emerging with Ubiquitous Computing ed by Ulrik Ekman MIT Press: Cambridge MA

Smith, Caspar Llewellyn (March 3 2014) "Ellen DeGeneres' Oscars Selfie Beats Obama Retweet Record on Twitter” theguardian.com; Posted March 32014 04.05 GMT Last Accessed: April $4^{\text {th }} 2014$ URL: http://www.theguardian.com/film/2014/mar/03/ellen-degeneres-selfie-retweet-obama

Soroka, V., \& Rafaeli, S. (2006). Invisible participants. In Proceedings of the 15th international conference on World Wide Web - WWW '06 (p. 163). New York, New York, USA: ACM Press. doi:10.1145/1135777.1135806

Stone, M. (2009). Information Visualization: Challenge for the Humanities. In K. Smith, B. Leney, \& A. Friedlander (Eds.), Working Together or Apart: Promoting the Next Generation of Digital Scholarship (review) (pp. 43-56). Washington, DC: Council on Library and Information Resources. Retrieved from http://muse.jhu.edu/journals/pla/summary/v009/9.4.mower.html

Strate, L., Jacobson, R. L., \& Gibson, S. B. (2003). Communication and cyberspace: social interaction in an electronic environment. Hampton Press. Retrieved from http://books.google.ca/books?id=7P9oAAAAIAAJ

Waitt, Gordon (2010) "Doing Foucauldian Discourse Analysis - Revealing Social Realities" in Qualitative Research Methods in Human Geography edited by Iain Hay; Oxford University Press: Don Mills, ON

Walker, Stephen and Grytsenko, Oksana (January 21, 2014) “Text messages warn Ukraine protesters they are 'participants in mass riot"' thegaurdian Last Accessed: March 312014

http://www.theguardian.com/world/2014/jan/21/ukraine-unrest-text-messages-protesters-mass-riot 
Walther, J. (1996). Computer-mediated communication impersonal, interpersonal, and hyperpersonal interaction. Communication research, 23(1), 3-43. Retrieved from http://crx.sagepub.com/content/23/1/3.short

Walther, J. B., Loh, T., \& Granka, L. (2005). Let Me Count the Ways: The Interchange of Verbal and Nonverbal Cues in Computer-Mediated and Face-to-Face Affinity. Journal of Language and Social Psychology, 24(1), 36-65. doi:10.1177/0261927X04273036

Walther, J. B. (2009). "Theories, Boundaries, and All of the Above" in Journal of Computer-Mediated Communication, 14(3), 748-752. doi:10.1111/j.1083-6101.2009.01466.x

Waterworth, John A. (1992) Multimedia Interaction with Computers: Human Factors Issues Ellis Horwood: Toronto, ON

Yahoo! Canada News (March 26, 2013) "Did Stephen Harper slight the Nishiyuu Walkers?" in Pulse of Canada (Comments section) Last Accessed April 4 ${ }^{\text {th }} 2013$ URL: https://ca.news.yahoo.com/blogs/pulseofcanada/did-stephen-harper-slight-nishiyuu-walkers$\underline{145121229 . \mathrm{html}}$

@jianghomeshi (March 25 2013) “The \#Nishiyuu walkers are a true inspiration. And deserve more attention than visiting pandas. - my opening essay tmrw morn. \#Q" Twitter.com [Tweet] Last Accessed April 42013 URL: https://twitter.com/jianghomeshi/status/316312362632216576

@marymacjess (March 25 2013) "Pandas are cool and all, but aren't there more important things to pay attention to, Mr. Harper? Like \#Nishiyuu perhaps?” Twitter.com [Tweet] Last Accessed April 4 2013 URL: https://twitter.com/marymacjess/status/316288798092828673

@ Nishiyuu (March 25 2013, 8:32AM) "Now arriving on Victoria Island... We can't thank you enough for your support! \#Nishiyuu \#March25 \#Nishiyuuarrivalday \#Unity \#Idlnomore” Twitter.com [Tweet] Last accessed March 302014 URL: https://twitter.com/Nishiiyuu/status/316211025680670720

@ Nishiyuu (March 25 2013) “\#Unity has been witnessed on the \#Journey from all walks of life! We thank you with \#Love. \#Nishiyuu" Twitter.com [Tweet] Last Accessed April 42013 URL: https://twitter.com/Nishiiyuu/status/315955885417590784

@onthebeat1 and @ryanpaulgibson (March 25 2013) "Journey of Nishiyuu comes to an end" [Live Tweet Stream] live.cbc.ca Last accessed March 172014 URL: http://live.cbc.ca/Event/Journey_of_Nishiyuu_comes_to_an_end

@rabble.ca (March 25 2013) “\#Nishiyuu is trending in Canada. Here's some background about this epic journey http://rabble.ca/news/2013/03/indigenous-youth-epic-journey-ottawa-deserve-attention- 
and-respect ... \#IdleNoMore \#cdnpoli” Twitter.com [Tweet] Last accessed: November 112013 URL: https://twitter.com/rabbleca/status/316268937505554434

“\#Nishiyuu” (November 11 2013) [Social Search] topsy.com Last accessed: January 132014 URL: http://topsy.com/s?q=\%23nishiyuu\&mintime $=1388599237$ 